Prepared in cooperation with the U.S. Army Corps of Engineers

\title{
A Multi-Year Analysis of Passage and Survival at McNary Dam, 2004-09
}

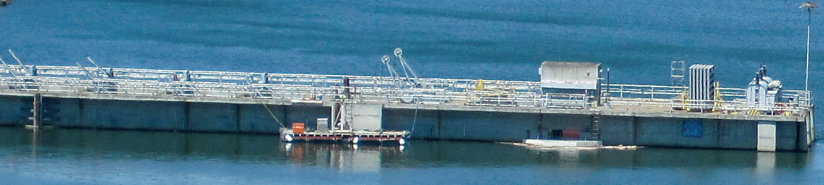

Open-File Report 2011-1230

U.S. Department of the Interior

U.S. Geological Survey 
Cover: Photograph of McNary Dam on the Columbia River, near Umatilla, Oregon. (Photograph taken by Scott Brewer, U.S. Geological Survey, Cook, Washington.) 


\section{A Multi-Year Analysis of Passage and Survival at McNary Dam, 2004-09}

By Noah S. Adams, Christopher E. Walker, and Russell W. Perry

Prepared in cooperation with the U.S. Army Corps of Engineers

Open-File Report 2011-1230 


\section{U.S. Department of the Interior \\ KEN SALAZAR, Secretary}

\section{U.S. Geological Survey \\ Marcia K. McNutt, Director}

U.S. Geological Survey, Reston, Virginia: 2011

For more information on the USGS-the Federal source for science about the Earth, its natural and living resources, natural hazards, and the environment, visit http://www.usgs.gov or call 1-888-ASK-USGS.

For an overview of USGS information products, including maps, imagery, and publications, visit http://www.usgs.gov/pubprod

To order this and other USGS information products, visit http://store.usgs.gov

Suggested citation:

Adams, N.S., Walker, C.E, and Perry, R.W., 2011, A multi-year analysis of passage and survival at McNary Dam, 2004-09: U.S. Geological Survey Open-File Report 2011-1230, 128 p.

Any use of trade, product, or firm names is for descriptive purposes only and does not imply endorsement by the U.S. Government.

Although this report is in the public domain, permission must be secured from the individual copyright owners to reproduce any copyrighted material contained within this report. 


\section{Contents}

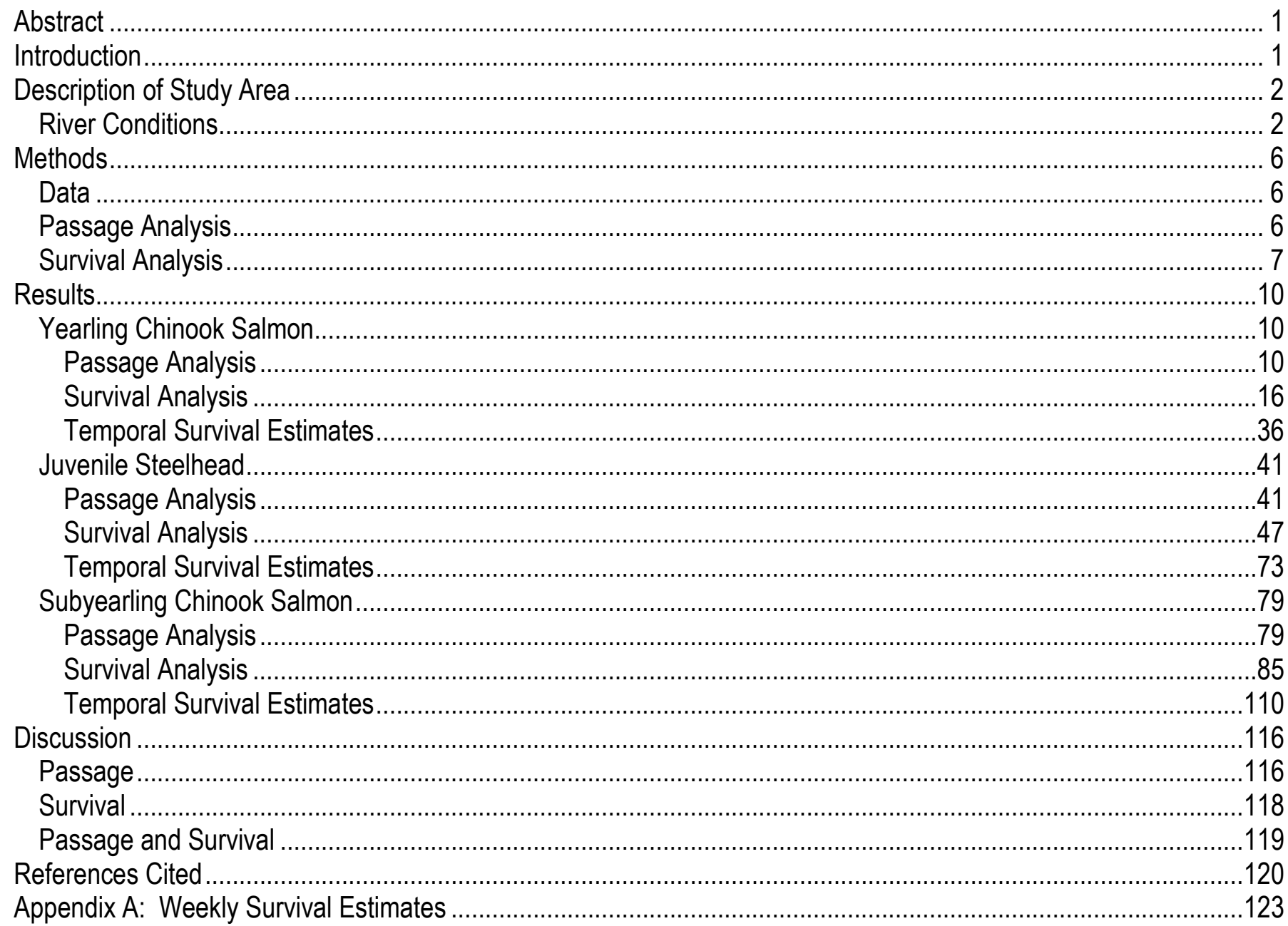




\section{Figures}

Figure 1. Map showing Columbia and Snake Rivers and location of McNary Dam relative to other major hydroelectric projects in the region.

Figure 2. Hydrograph of mean daily project outflow during radio and acoustic telemetry study dates at McNary Dam, 2004-09, and the 10-year average, 2000-2009

Figure 3. Hydrograph of mean daily project spill during radio and acoustic telemetry study dates at McNary Dam, 2004-09, and the 10-year average, 2000-2009.

Figure 4. Hydrograph of mean daily water temperature of the Columbia River at McNary Dam during radio and acoustic telemetry study dates, 2004-09, and the 10-year average, 2000-2009.

Figure 5. Schematic of the mark-recapture model used to estimate survival $(\varnothing)$ and detection probabilities $(p)$ of juvenile salmonids passing through McNary Dam for releases made during 2004-09...

Figure 6. Passage probabilities of yearling Chinook salmon predicted by continuous covariates at McNary Dam through the spillway, turbines, and the juvenile bypass system in relation to total discharge and percent spill during day (grey symbols) and night (black symbols) photoperiods, 2004-09.

Figure 7. Passage probabilities of yearling Chinook salmon related to total discharge and percent spill at McNary Dam through the spillway, turbines, and the juvenile bypass, 2004-09.

Figure 8. Passage probabilities of yearling Chinook salmon predicted by continuous covariates at McNary Dam through the juvenile bypass system in relation to fish weight, 2004-09.

Figure 9. Survival of yearling Chinook salmon migrating through the forebay of McNary Dam when temporary spillway weirs were not installed (solid line, 2005 and 2006) and were installed (dashed line, 2007-09) in relation to water temperature, total discharge, and fish weight.

Figure 10. Survival of yearling Chinook salmon that passed through turbines at McNary Dam in relation to water temperature, tag burden (tag weightffish weight $\times 100$ ), and fish weight during day (dashed line) and night (solid line) photoperiods, 2004-09.

Figure 11. Survival of yearling Chinook salmon that passed through the north (solid line) and south (dashed line) portion of the spillway at McNary Dam in relation to total discharge, fish weight, and tag burden (tag burden=tag weight/fish weight $\times 100,2004-09$ 33

Figure 12. Weekly survival of yearling Chinook salmon in the forebay of McNary Dam, 2005-09. 37

Figure 13. Weekly survival of yearling Chinook salmon for fish that passed through the juvenile bypass system, spillway, turbines, or that were released in the tailrace of McNary Dam, 2004-09.

Figure 14. Observed versus predicted weekly (left) and annual survival (right) of yearling Chinook salmon during the spring migration period in the forebay of McNary Dam, 2005-09.

Figure 15. Observed versus predicted weekly (top) and annual survival (bottom) of yearling Chinook salmon during the spring migration period for fish that passed through the turbines (left) and spillway (right) of McNary Dam, 2004-09.

Figure 16. Passage probabilities of juvenile steelhead predicted by continuous covariates at McNary Dam through the spillway, turbines, and the juvenile bypass system in relation to total discharge and percent spill during day (grey symbols) and night (black symbols) photoperiods, 2004-09

Figure 17. Passage probabilities of juvenile steelhead related to total discharge and percent spill at McNary Dam through the spillway, turbines, and the juvenile bypass system, 2004-09.

Figure 18. Passage probabilities of juvenile steelhead predicted by continuous covariates at McNary Dam through the spillway in relation to fish weight, 2004-09.

Figure 19. Passage probabilities of juvenile steelhead predicted by continuous covariates at McNary Dam through the spillway, turbines, and the juvenile bypass system in relation to water temperature during day (grey symbols) and night (black symbols) photoperiods, 2004-09 
Figure 20. Survival of juvenile steelhead migrating through the forebay of McNary Dam during day (dashed line) and night (solid line) time photoperiods in relation to water temperature, total discharge, and percent spill......

Figure 21. Survival of juvenile steelhead that passed through turbines at McNary Dam in relation to head (forebay elevation - tailrace elevation) and total discharge during day (dashed line) and night (solid line) photoperiods, 2004-09

Figure 22. Survival of juvenile steelhead that passed through the juvenile bypass system at McNary Dam in relation to temperature and total discharge, 2004-09. The dashed lines represent the 95-percent confidence limits

Figure 23. Survival of juvenile steelhead that passed through the north (solid line) and south (dashed line) portion of the spillway at McNary Dam in relation to temperature, total discharge, and percent spill, 2004-09..........66 Figure 24. Survival of juvenile steelhead that passed through the north (solid line) and south (dashed line) portion of the spillway at McNary Dam in relation to fish weight and tag weight, 2004-09

Figure 25. Survival of juvenile steelhead that were released in the tailrace of McNary Dam related to total discharge and percent spill during day (dashed line) and night (solid line) photoperiods, 2004-09.....

Figure 26. Survival of juvenile steelhead released in the tailrace of McNary Dam in relation to discharge and percent spill at varying levels during the day photoperiod, 2004-09

Figure 27. Survival of juvenile steelhead released in the tailrace of McNary Dam in relation to discharge and percent spill at varying levels during the night photoperiod, 2004-09.

Figure 28. Weekly survival of juvenile steelhead in the forebay of McNary Dam, 2005-09 74

Figure 29. Weekly survival of juvenile steelhead that passed through the juvenile bypass system, spillway, turbines, or that were released in the tailrace of McNary Dam, 2004-09

Figure 30. Observed versus predicted weekly (left) and annual survival (right) of juvenile steelhead during the spring migration period in the forebay of McNary Dam, 2005-09...

Figure 31. Observed versus predicted weekly survival of juvenile steelhead that passed through the juvenile bypass system, spillway, turbines, or that were released in the tailrace of McNary Dam, 2004-09 ....

Figure 32. Observed versus predicted annual survival of juvenile steelhead during the spring migration period for fish that passed through the juvenile bypass system, spillway, turbines, or that were released in the tailrace of McNary Dam, 2004-09....

Figure 33. Passage probabilities of subyearling Chinook salmon predicted by continuous covariates at McNary Dam through the spillway, turbines, and the juvenile bypass system in relation to total discharge and percent spill during day (grey symbols) and night (black symbols) photoperiods, 2004-09.....

Figure 34. Passage probabilities of subyearling Chinook salmon predicted by continuous covariates at McNary Dam through the spillway, turbines, and the juvenile bypass system in relation to water temperature during day (grey symbols) and night (black symbols) photoperiods, 2004-09.

Figure 35. Passage probabilities of subyearling Chinook salmon predicted by continuous covariates at McNary Dam through the spillway, turbines, and juvenile bypass system in relation to fish weight, 2004-09

Figure 36. Passage probabilities of subyearling Chinook salmon related to total discharge and percent spill at McNary Dam through the spillway, turbines, and the juvenile bypass, 2004-09

Figure 37. Survival of subyearling Chinook salmon migrating through the forebay of McNary Dam in relation to water temperature, total discharge, and percent spill, 2004-09.

Figure 38. Survival of subyearling Chinook salmon migrating through the forebay of McNary Dam in relation to total discharge, percent spill, and their interaction, 2004-09

Figure 39. Survival of subyearling Chinook salmon passing through turbines at McNary Dam in relation to water temperature, 2004-09.

Figure 40. Survival of subyearling Chinook salmon passing through turbines at McNary Dam in relation to percent spill and discharge, 2004-09

Figure 41. Survival of subyearling Chinook salmon passing through the juvenile bypass system at McNary Dam in relation to water temperature, 2004-09 
Figure 42. Survival of subyearling Chinook salmon passing through the spillway at McNary Dam in relation to water temperature, discharge, percent spill, and tag burden, 2004-09.....

Figure 43. Survival of subyearling Chinook salmon released in the tailrace of McNary Dam in relation to water temperature during day (dashed line) and night (solid line) photoperiods, 2004-09. 109

Figure 44. Weekly survival of subyearling Chinook salmon in the forebay of McNary Dam, 2004-09

Figure 45. Weekly survival of subyearling Chinook salmon for fish that passed through the juvenile bypass system, spillway, turbines, or that were released in the tailrace of McNary Dam, 2004-09

Figure 46. Observed versus predicted weekly (left) and annual survival (right) of subyearling Chinook salmon during the summer migration period in the forebay of McNary Dam, 2005-09

Figure 47. Observed versus predicted weekly survival of subyearling Chinook salmon for fish that passed through the juvenile bypass system, spillway, turbines, or that were released in the tailrace of McNary Dam, 2004-09

Figure 48. Observed versus predicted annual survival of subyearling Chinook salmon during the summer migration period for fish that passed through the juvenile bypass system, spillway, turbines, or that were released in the tailrace of McNary Dam, 2004-09....

\section{Tables}

Table 1. Sample sizes of fish used in this analysis that passed or were released in the tailrace of McNary Dam, 2004-09.

Table 2. List of variables used in analyses of survival for fish passing McNary Dam and for those released in the tailrace, 2004-09

Table 3. Passage models for yearling Chinook salmon that passed through McNary Dam, 2004-09, comparing the full model with models that have one variable removed. 11

Table 4. Slope (Beta) coefficients, standard error, and 95-percent confidence limits of model parameters of passage probabilities for yearling Chinook salmon passing McNary Dam, 2004-09.....

Table 5. Model selection results based on varying $\lambda$ and $p$ with respect to year for yearling Chinook salmon, 2004-09

Table 6. Model selection for fish that passed through the forebay at McNary Dam relating survival (ø) as a function of environmental variables using the fixed structure of the best model for $p$ and $\lambda$ parameters for yearling Chinook salmon, 2005-09.

Table 7. Slope (Beta) coefficients, standard error, and 95-percent confidence limits of model parameters for yearling Chinook salmon passing McNary Dam, 2004-09

Table 8. Model selection for fish that passed through turbines at McNary Dam relating survival (ø) as a function of environmental variables using the fixed structure of the best model for $p$ and $\lambda$ parameters for yearling Chinook salmon, 2004-09.

Table 9. Model selection for fish that passed through the juvenile bypass system at McNary Dam relating survival $(\varnothing)$ as a function of environmental variables using the fixed structure of the best model for $p$ and $\lambda$ parameters for yearling Chinook salmon, 2004-09

Table 10. Model selection for fish that passed through the spillway at McNary Dam relating survival ( $\varnothing)$ as a function of environmental variables using the fixed structure of the best model for $p$ and $\lambda$ parameters for yearling Chinook salmon, 2004-09.....

Table 11. Model selection for fish released in the tailrace of McNary Dam relating survival (ø) as a function of environmental variables using the fixed structure of the best model for $p$ and $\lambda$ parameters for yearling Chinook salmon, 2004-09.

Table 12. Annual survival estimates during the spring migration period for yearling Chinook salmon passing through and released in the tailrace of McNary Dam, 2004-09.

Table 13. Passage models for juvenile steelhead that passed through McNary Dam, 2004-09, comparing the full model with models that have one variable removed 
Table 14. Slope (Beta) coefficients, standard error, and 95-percent confidence limits of model parameters of passage probabilities for juvenile steelhead passing McNary Dam, 2004-09....

Table 15. Model selection results based on varying $\lambda$ and $p$ with respect to year for juvenile steelhead at McNary Dam, 2004-09.

Table 16. Model selection for fish that passed through the forebay at McNary Dam relating survival (ø) as a function of environmental variables using the fixed structure of the best model for $p$ and $\lambda$ parameters for juvenile steelhead, 2004-09.

Table 17. Model selection for fish that passed through turbines at McNary Dam relating survival (ø) as a function of environmental variables using the fixed structure of the best model for $p$ and $\lambda$ parameters for juvenile steelhead, 2004-09

Table 18. Slope (Beta) coefficients, standard error, and 95-percent confidence limits of model parameters for juvenile steelhead passing McNary Dam, 2004-09...

Table 19. Model selection for fish that passed through the juvenile bypass system at McNary Dam relating survival $(\varnothing)$ as a function of environmental variables using the fixed structure of the best model for $p$ and $\lambda$ parameters for juvenile steelhead, 2004-09

Table 20. Model selection for fish that passed through the spillway at McNary Dam relating survival (ø) as a function of environmental variables using the fixed structure of the best model for $p$ and $\lambda$ parameters for juvenile steelhead, 2004-09

Table 21. Model selection for fish released in the tailrace of McNary Dam relating survival (ø) as a function of environmental variables using the fixed structure of the best model for $p$ and $\lambda$ parameters for juvenile steelhead, 2004-09.

Table 22. Annual survival estimates during the spring migration period for juvenile steelhead passing through, and released in the tailrace of, McNary Dam, 2004-09.

Table 23. Passage models for subyearling Chinook salmon that passed through McNary Dam, 2004-09, comparing the full model with models that have one variable removed

Table 24. Slope (Beta) coefficients, standard error, and 95-percent confidence limits of model parameters of passage probabilities for subyearling Chinook salmon passing McNary Dam, 2004-09

Table 25. Model selection results based on varying $\lambda$ and $p$ with respect to year for subyearling Chinook salmon, 2004-09

Table 26. Model selection for fish that passed through the forebay at McNary Dam relating survival (ø) as a function of environmental variables using the fixed structure of the best model for $p$ and $\lambda$ parameters for subyearling Chinook salmon, 2004-09.

Table 27. Slope (Beta) coefficients, standard error, and 95-percent confidence limits of model parameters for subyearling Chinook salmon passing McNary Dam, 2004-09

Table 28. Model selection for fish that passed through turbines at McNary Dam relating survival $(\varnothing)$ as a function of environmental variables using the fixed structure of the best model for $p$ and $\lambda$ parameters for subyearling Chinook salmon, 2004-09.

Table 29. Model selection for fish that passed through the juvenile bypass system at McNary Dam relating survival ( $)$ as a function of environmental variables using the fixed structure of the best model for $p$ and $\lambda$ parameters for subyearling Chinook salmon, 2004-09.

Table 30. Model selection for fish that passed through the spillway at McNary Dam relating survival (ø) as a function of environmental variables using the fixed structure of the best model for $p$ and $\lambda$ parameters for subyearling Chinook salmon, 2004-09....

Table 31. Model selection for fish released in the tailrace of McNary Dam relating survival (ø) as a function of environmental variables using the fixed structure of the best model for $p$ and $\lambda$ parameters for subyearling Chinook salmon, 2004-09

Table 32. Annual survival estimates during the summer migration period for subyearling Chinook salmon passing through and released in the tailrace of McNary Dam, 2004-09. 


\section{Conversion Factors, Datums, Abbreviations, and Acronyms}

\section{Conversion Factors}

Inch/Pound to SI

\begin{tabular}{ccc}
\hline Multiply & By & To obtain \\
\hline cubic foot per second $\left(\mathrm{ft}^{3} / \mathrm{s}\right)$ & Flow rate & \\
\hline
\end{tabular}

SI to Inch/Pound

\begin{tabular}{lcl}
\hline Multiply & By & To obtain \\
\hline kilometer $(\mathrm{km})$ & 0.6214 & mile $(\mathrm{mi})$ \\
\hline Mass & \\
\hline $\operatorname{gram}(\mathrm{g})$ & 0.03527 & ounce, avoirdupois $(\mathrm{oz})$ \\
\hline
\end{tabular}

Temperature in degrees Celsius $\left({ }^{\circ} \mathrm{C}\right)$ may be converted to degrees Fahrenheit $\left({ }^{\circ} \mathrm{F}\right)$ as follows:

${ }^{\circ} \mathrm{F}=\left(1.8 \times{ }^{\circ} \mathrm{C}\right)+32$.

\section{Datums}

Horizontal coordinate information is referenced to the World Geodetic System of 1984 (WGS84).

Vertical coordinate information is referenced to the National Geodetic Vertical Datum of 1929 (NGVD 29).

\section{Abbreviations and Acronyms}

$\begin{array}{ll}\text { AIC } & \text { Akaike's Information Criterion } \\ \text { CH1 } & \text { yearling Chinook salmon (Oncorhynchus tshawytscha). } \\ \text { CH0 } & \text { subyearling Chinook salmon (Oncorhynchus tshawytscha). } \\ \text { CJS } & \text { Cormack-Jolly-Seber models } \\ \text { Forebay } & \text { area of Columbia River extending from McNary Dam to } 2 \text { km upstream. } \\ \text { PIT } & \text { passive integrated transponder. } \\ \text { Powerhouse } & \text { turbine and Bypass (units 1-14). } \\ \text { Spillway } & \text { conventional spill bays (bays 1-22 excluding bays } 20 \text { and } 22 \text { in 2007, 19 and 20 in 2008, 4 and 20 in spring 2009, } \\ & \text { and 19 and 20 in summer 2009). } \\ \text { STH } & \text { steelhead (Oncorhynchus mykiss). } \\ \text { Tailrace } & \text { area of Columbia River extending from McNary Dam to } 2.4 \text { km downstream. } \\ \text { TSW } & \text { temporary spillway weir } \\ \text { USACE } & \text { U.S. Army Corps of Engineers. } \\ \text { USGS } & \text { U.S. Geological Survey } \\ \text { rkm } & \text { river kilometer } \\ \text { kcfs } & 1,000 \mathrm{ft}^{3} / \mathrm{s}\end{array}$




\title{
A Multi-Year Analysis of Passage and Survival at McNary Dam, 2004-09
}

\author{
By Noah S. Adams, Christopher E. Walker, and Russell W. Perry
}

\begin{abstract}
We analyzed 6 years (2004-09) of passage and survival data collected at McNary Dam to determine how dam operations and environmental conditions affect passage and survival of juvenile salmonids. A multinomial logistic regression was used to examine how environmental variables and dam operations relate to passage behavior of juvenile salmonids at McNary Dam. We used the Cormack-Jolly-Seber release-recapture model to determine how the survival of juvenile salmonids passing through McNary Dam relates to environmental variables and dam operations. Total project discharge and the proportion of flow passing the spillway typically had a positive effect on survival for all species and routes. As the proportion of water through the spillway increased, the number of fish passing the spillway increased, as did overall survival. Additionally, survival generally was higher at night. There was no meaningful difference in survival for fish that passed through the north or south portions of the spillway or powerhouse. Similarly, there was no difference in survival for fish released in the north, middle, or south portions of the tailrace. For subyearling Chinook salmon migrating during the summer season, increased temperatures had a drastic effect on passage and survival. As temperature increased, survival of subyearling Chinook salmon decreased through all passage routes and the number of fish that passed through the turbines increased. During years when the temporary spillway weirs (TSWs) were installed, passage through the spillway increased for spring migrants. However, due to the changes made in the location of the TSW between years and the potential effect of other confounding environmental conditions, it is not certain if the increase in spillway passage was due solely to the presence of the TSWs. The TSWs appeared to improve forebay survival during years when they were operated.
\end{abstract}

\section{Introduction}

As juvenile salmon Oncorhynchus spp. and steelhead O. mykiss migrate downriver to the ocean, they are subject to both natural- and human-induced mortality. Avian and piscivorous predators contribute to total natural mortality along with other factors (Vigg and others, 1991; Collis and others, 2001). Hydroelectric projects on the Snake and Columbia Rivers also can be major sources of mortality for migrating juvenile fish. Impoundments caused by dams may indirectly contribute to salmonid mortality by slowing the migration of juvenile salmonids (Raymond 1968, 1979; Plumb and others, 2006), thereby increasing energy expenditure during migration and allowing greater opportunity for predation. Furthermore, passage through dams can be a major source of direct mortality (Mesa, 1994; Whitney and others, 1997) that is cumulative for salmonid populations that negotiate multiple dams.

Studies monitoring fish movements near McNary Dam were conducted from 2004 to 2009 to assess how dam operations or fish passage structures influence passage and survival of juvenile fish migrating through the hydroelectric system in a particular year. Although the annual studies provided 
valuable information for developing management strategies, some important questions remain unanswered. Managers are often interested in understanding how survival and passage varies over a range of environmental conditions, such as total river discharge or distribution of discharge across passage routes. Constant conditions are favorable when the goal is point estimation under a given condition, but understanding how survival or passage varies in response to dam operations requires data over a wide range of conditions. Studies conducted in any given year must deal with the environmental conditions that nature delivers, which often leads to a narrow range of study conditions within a single year. Analyzing data across multiple years is better suited to developing quantitative relationships than are annual studies, because operational and environmental variation typically will be higher over a 5-10 year period than within any given year. Furthermore, multi-year analyses benefit from the large sample sizes, which can reduce statistical uncertainty and help to identify relationships that might otherwise be statistically undetectable. We analyzed 6 years (2004-09) of passage and survival data collected at McNary Dam to determine how dam operations and environmental conditions affect passage and survival of juvenile salmonids.

\section{Description of Study Area}

McNary Dam is the fourth dam on the Columbia River, located 470 river kilometers (rkm) upriver from the Pacific Ocean and 52 rkm downriver from the confluence of the Columbia and Snake Rivers (fig. 1). The reservoir formed by McNary Dam (Lake Wallula) extends $98 \mathrm{rkm}$ upriver to the Hanford Reach of the Columbia River, and impounds 16 rkm of the Snake River downstream of Ice Harbor Dam. The river downstream of McNary Dam (Lake Umatilla) is impounded by John Day Dam located $123 \mathrm{rkm}$ downriver of McNary Dam. Our study area extended from Hat Rock State Park, Oregon, $10 \mathrm{rkm}$ upriver of McNary Dam, to Sundale, Washington, $92 \mathrm{rkm}$ downstream of McNary Dam, where our last detection array was located.

McNary Dam is oriented perpendicular to the river channel and includes a navigation lock, spillway, powerhouse, and earthen dam. The spillway is 399-m-long with 22 vertical lift-type spill gates that regulate discharge through the dam. The spillway discharges water at the ogee crest approximately $14 \mathrm{~m}$ below the water surface. During the sampling seasons in years $2007-09$, temporary spillway weirs (TSWs) were installed as part of a strategy to improve fish passage at the dam.

\section{River Conditions}

Average daily discharge throughout a season was variable depending upon year. The 10-year average (2000-09) in mid-April was about $210 \mathrm{kcfs}$, with discharge increasing to greater than $250 \mathrm{kcfs}$ by late May (fig. 2). River-flows for the 10-year average decreased through June and July, ending less than $150 \mathrm{kcfs}$ by August. Average daily spill at McNary Dam from 2000 to 2009 followed a similar trend to mean daily outflow (fig. 3). Mean daily spill in mid-April, at the start of the season, averaged $80 \mathrm{kcfs}$ and peaked in late May or early June at $125 \mathrm{kcfs}$ for the 10-year average. During 2004 and 2005 , flow through the dam was low compared to the 10-year average. In 2004, very little flow was discharged through the spillway during the summer season. More detailed information regarding average daily discharge, including how discharge varied during the day and night periods, can be found in the annual reports of research.

Water temperature steadily increased during the study period, from $9^{\circ} \mathrm{C}$ in April to a peak of about $21^{\circ} \mathrm{C}$ in late July or early August (fig. 4). Water temperatures were slightly lower $\left(1-2^{\circ} \mathrm{C}\right)$ in 2008 than during the other 5 study years. For more detailed information on the environmental conditions and dam operations for individual years included in this analysis, please refer to Adams and Evans (2011). 


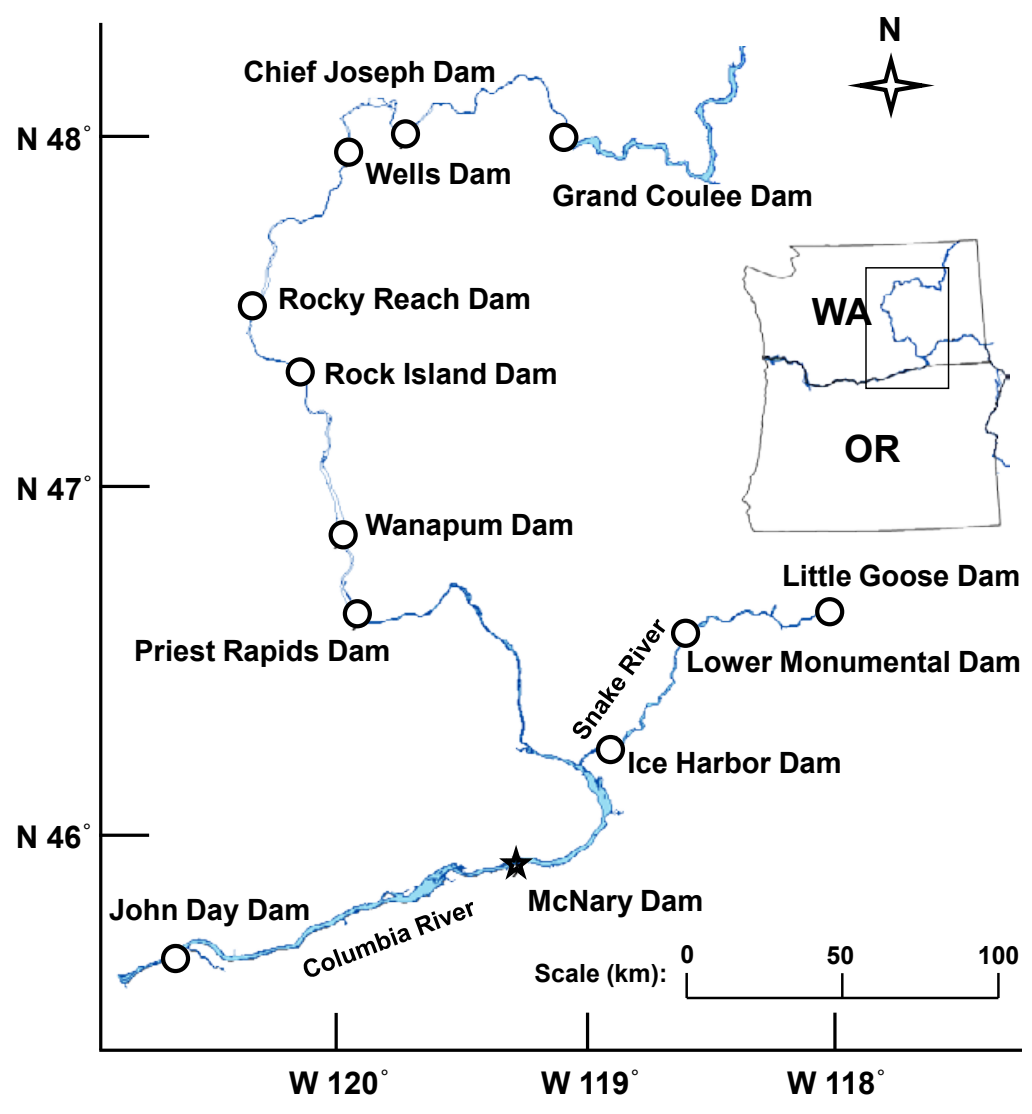

Figure 1. Map showing Columbia and Snake Rivers and location of McNary Dam relative to other major hydroelectric projects in the region. 


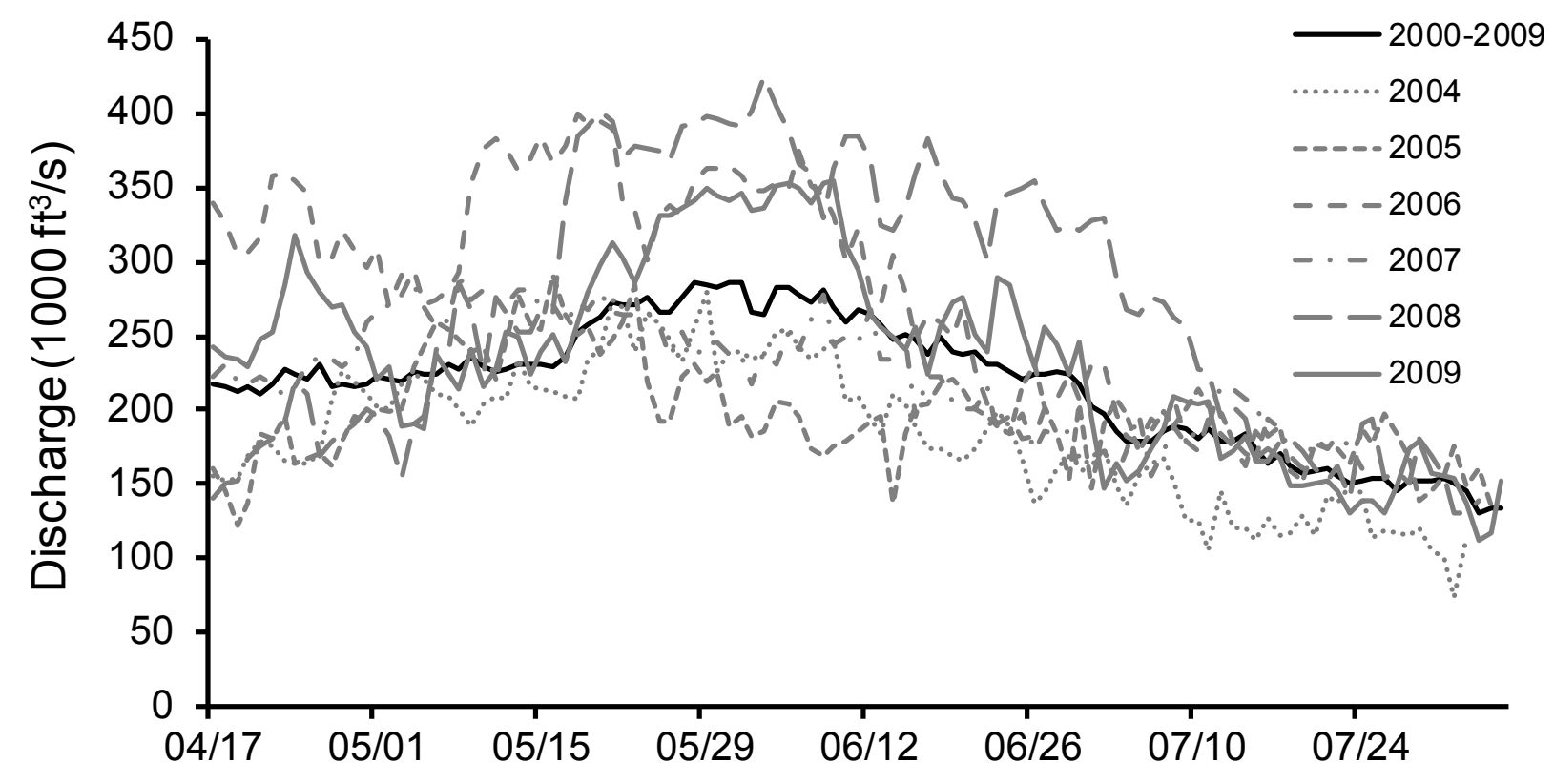

Figure 2. Hydrograph of mean daily project outflow during radio and acoustic telemetry study dates at McNary Dam, 2004-09, and the10-year average, 2000-2009. Data obtained from Columbia River DART website: http://www.cbr.washington.edu/dartriver.html.

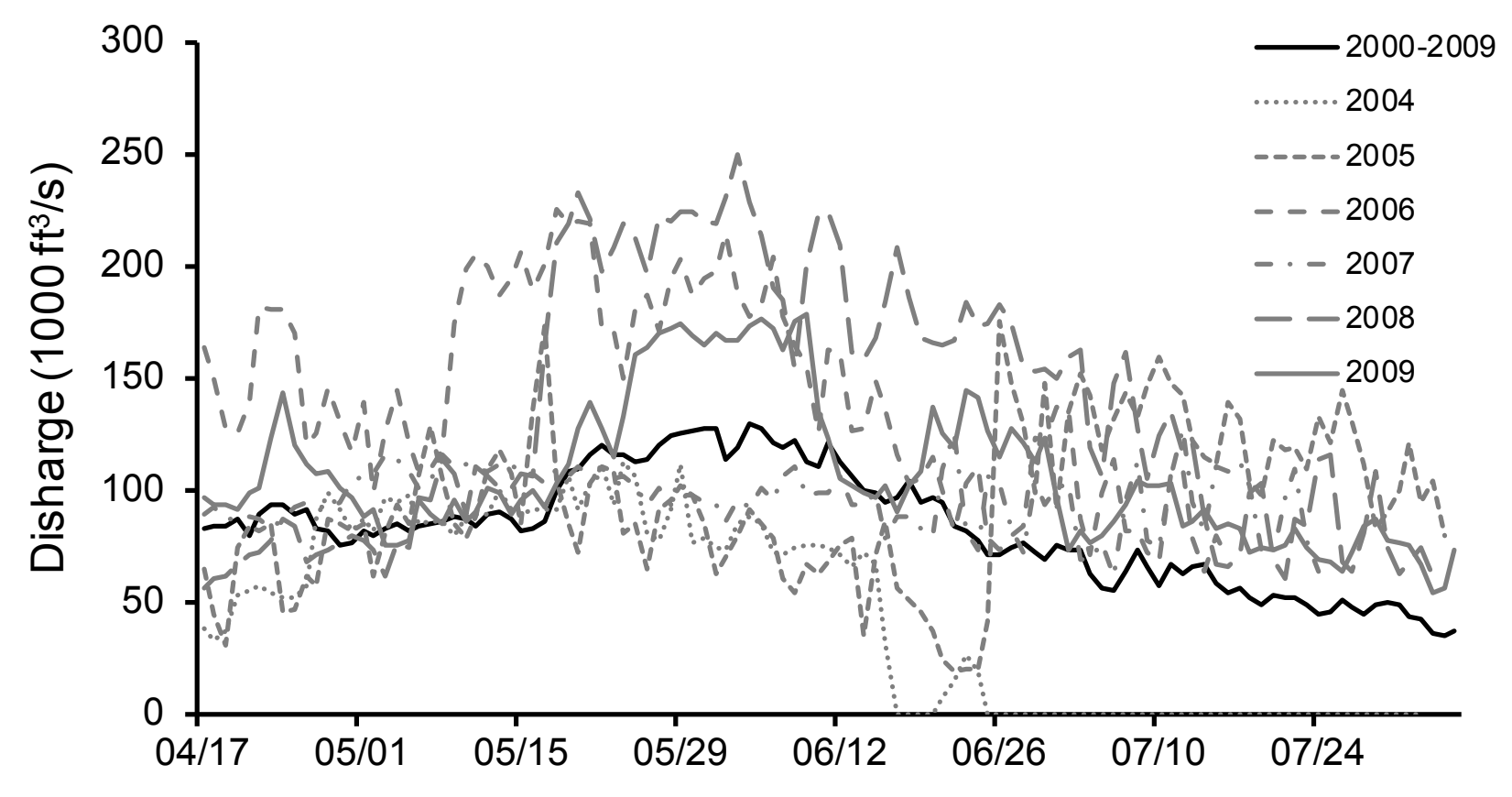

Figure 3. Hydrograph of mean daily project spill during radio and acoustic telemetry study dates at McNary Dam, 2004-09, and the 10-year average, 2000-2009. Data obtained from Columbia River DART website: http://www.cbr.washington.edu/dartriver.html. 


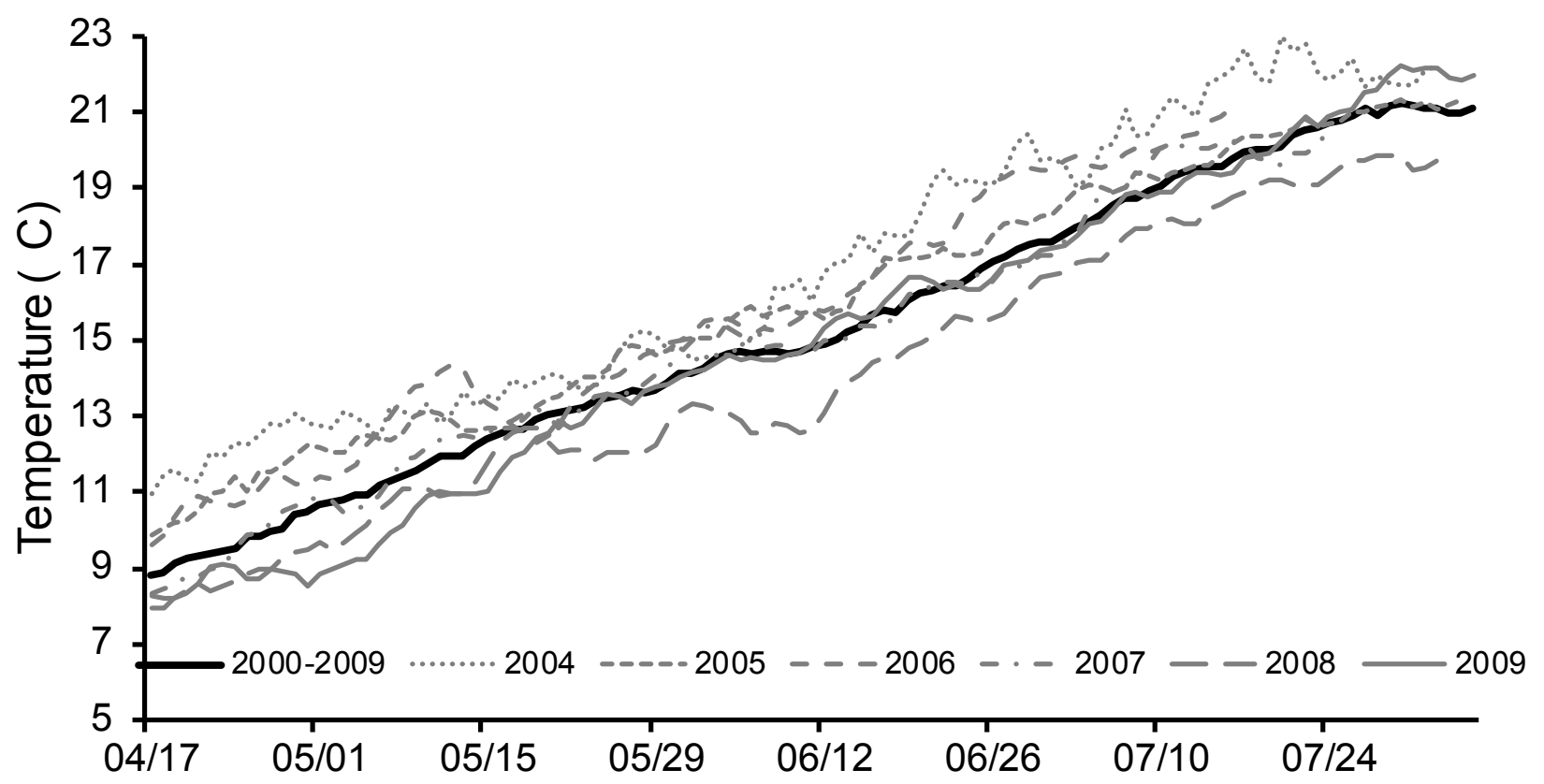

Figure 4. Hydrograph of mean daily water temperature of the Columbia River at McNary Dam during radio and acoustic telemetry study dates, 2004-09, and the 10-year average, 2000-2009. Data obtained from Columbia River DART website: $h$ ttp://www.cbr.washington.edu/dartriver.html. 


\section{Methods}

\section{Data}

We analyzed radio and acoustic telemetry data collected during the spring and summer seasons of 2004 through 2009 to investigate factors that might influence passage and survival of juvenile salmonids at McNary Dam. Telemetry equipment was deployed upstream, at, and downstream of McNary Dam to monitor fish movements. During the years included in this analysis, more than 42,000 yearling Chinook salmon, juvenile steelhead, and subyearling Chinook salmon were obtained from the juvenile fish collection and bypass facility at McNary Dam (table 1) and implanted with transmitters. Yearling Chinook salmon and juvenile steelhead were hatchery-reared and subyearling Chinook salmon were of unknown origin. Fish were released $10 \mathrm{~km}$ upstream of McNary Dam and about $0.5 \mathrm{~km}$ downstream of the dam. Details on the methods used to tag fish and collect the data are given in Adams and others (1998, 2008), Adams and Counihan (2009), Perry and others (2006, 2007), and Adams and Liedtke (2010). Survival was assessed from dam passage to a downstream array located at rkm 446-448.

Table 1. Sample sizes of fish used in this analysis that passed or were released in the tailrace of McNary Dam, 2004-09.

\begin{tabular}{lccc}
\hline \multicolumn{1}{c}{ Route } & Yearling Chinook salmon & Juvenile steelhead & Subyearling Chinook salmon \\
\hline Forebay & 6,841 & 3,912 & 5,966 \\
Turbine & 1,301 & 354 & 1,971 \\
Bypass & 2,888 & 1,397 & 1,853 \\
Spillway & 5,452 & 4,309 & 4,903 \\
Tailrace/Control & 6,736 & 2,316 & 6,547 \\
\hline
\end{tabular}

\section{Passage Analysis}

To examine how environmental variables and dam operations affect the probability that fish pass a particular route at McNary Dam, a multinomial logistic regression was used. A multinomial logit link was used to relate individual and group covariates (table 2) of environmental variables assigned at passage to passage probabilities and were estimated using maximum likelihood techniques. To accomplish this, the R software (R Development Core Team, 2007) was used to estimate parameters using an optimization (optim in R) routine. We formed an initial full model that included all possible explanatory variables and then eliminated variables that failed to improve model fit to the data. Each variable was removed one-at-a-time from the full model, fit to the data, and a likelihood ratio test was used to determine whether removing the variable resulted in a significantly poorer fit of the model to the data. The variable with the largest $P$-value was eliminated from the model, a new model was formed, and variables were again removed one-at-a-time and fit to the data. This process was repeated until no further variables could be removed from the model at $a=0.05$. Detection probabilities were not incorporated into this analysis because they were very high at the dam for all routes and years averaging 0.985 . 


\section{Survival Analysis}

To examine how environmental variables and dam operations affect survival of juvenile salmonids passing through McNary Dam, we used Cormack-Jolly-Seber release-recapture models (CJS) developed by Cormack (1964), Jolly (1965), and Seber (1965). Further, a logit link was used to relate individual and group covariates of environmental variables assigned at forebay entry, dam passage, and release time (table 2) to survival in the forebay, passage route, and tailrace, respectively. To accomplish this, the R software (R Development Core Team, 2011) and RMark package (Laake and Rextad, 2008) were used to create models for the program MARK (White and Burnham, 1999). Group covariates for photoperiod were determined by using civil twilight for each day. Furthermore, group covariates for passage location were defined based on gear consistencies across years so that fish were assigned as passing through a particular part of the spillway or powerhouse (that is, north, south). Fish passing through spill bays 1-15 were defined as passing north and fish passing through bays 16-20 were defined as passing south (table 2). For the powerhouse, north was defined for fish passing through units 8-14 and south was defined for fish passing units 1-7.

Table 2. List of variables used in analyses of survival for fish passing McNary Dam and for those released in the tailrace, 2004-09.

[Tag burden was used as a secondary variable in lieu of fish weight after all other variables were evaluated due to high correlation. Head was used as a secondary variable in lieu of discharge after all other variables were evaluated due to high correlation. Year and TSW were used as secondary variables to assess if additional variation could be explained temporally]

\begin{tabular}{llll}
\hline Type & Name & Definition & Note \\
\hline Group & Photoperiod & Day, Night & \\
Group & Year & yyyy & Secondary \\
Group & PassLoc & North or South passage location at powerhouse or spillway & \\
Group & TSW & Not installed (0) or Installed (1) & Secondary \\
Individual & Temperature & Average daily tailrace water temperature & \\
Individual & Discharge & River discharge & Secondary \\
Individual & Head & Forebay elevation - tailrace elevation & \\
Individual & \%Spill & Percent discharge through spillway & \\
Individual & Weight & Fish weight & Secondary \\
Individual & Tag Weight & Tag weight & Secondary \\
Individual & Tag Burden & Tag weight/fish weight & \\
\hline
\end{tabular}

An initial step in the modeling process was to assign probabilities to detection histories that represent detections of fish downstream. For example, the possible histories for survival in this study included: $111,101,110$, and 100 , where a ' 1 ' represents detection at a telemetry array and a ' 0 ' represents non-detection. The first digit represents a fish that was released or passed through a particular passage route, the second digit represents the first downstream detection array, and the last digit represents detection at the last detection array. The probabilities of these capture histories were then incorporated into a multinomial probability model to estimate model parameters using maximum likelihood methods. The model parameters estimated as probabilities using the detection history information with a CJS model include: $\varnothing$, the probability of survival in the forebay or from dam passage 
through a route to the first downstream detection array; $p$, the probability of being detected at the first downstream detection array given the individual survived; and $\lambda$, the joint probability of surviving and being detected from the first downstream array to the next downstream array (fig. 5). The probabilities of the possible detection histories can be expressed as:

$P_{111}: \varnothing p \lambda$

$P_{101}: \varnothing(1-p) \lambda$

$P_{110}: \varnothing p(1-\lambda)$

$P_{100}:(1-\varnothing)+\varnothing(1-p)(1-\lambda),(1)$

For example, to express survival as a function of a photoperiod effect and a continuous covariate $x$, the following expression is used;

$\varnothing($ photo $+x i)=\exp \left(\beta_{0}+\beta_{\text {photo }}+\beta_{1} x i\right) / 1+\exp \left(\beta_{0}+\beta_{\text {photo }}+\beta_{1} x i\right),(2)$

In this expression, $\beta$ represents the intercept and slope coefficients. The logit link was also used for estimating our detection parameters. The logit link was used to estimate all model parameters because it constrains parameter estimates between 0 and 1 which is appropriate for probabilities. A quadratic term was included in models where there was potential for a unimodal curvilinear response of fish survival to a particular covariate. We found that adding this term to the models never resulted in a significant effect, and as such, we do not present the results.

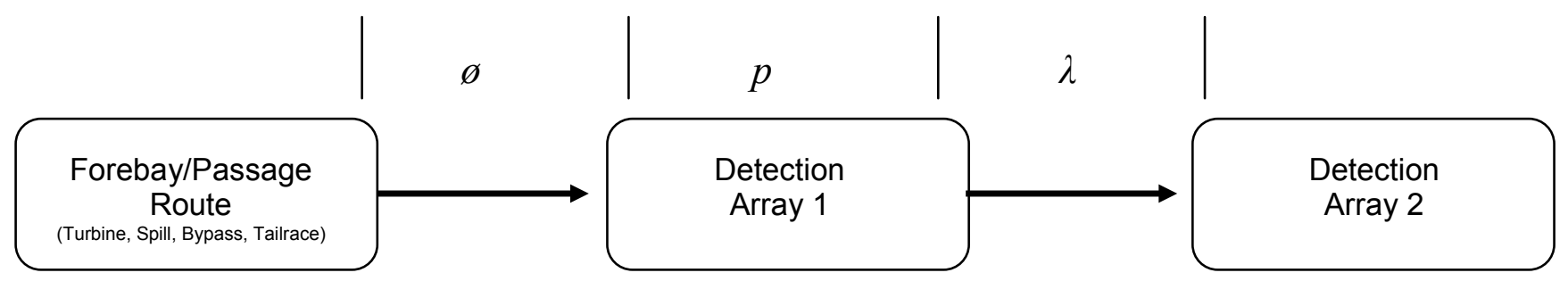

Figure 5. Schematic of the mark-recapture model used to estimate survival $(\varnothing)$ and detection probabilities $(p)$ of juvenile salmonids passing through McNary Dam for releases made during 2004-09.

To assess how well the covariates or environmental variables explained model parameters, Akaike's Information Criterion (AIC; Akaike, 1973; Burnham and Anderson, 1998) was used for comparing models. AIC allowed us to measure how well the model fit relative to the number of parameters used in the model:

$$
A I C=-2 L+2 N,(3)
$$

where $N$ is the number of parameters and $L$ is the log-likelihood. Although our sample sizes were large, we used $\mathrm{AIC} c$

$$
A I C c=-2 L+2 N+(2 N(N+1) /(n-N-1)),(4)
$$

which is a modification for small sample sizes in relation to the number of parameters in a model where $n$ is sample size. When sample sizes are large in relation to the number of parameters in a model, AIC $c$ is equivalent to $\mathrm{AIC}$; thus, $\mathrm{AIC} c$ provides more flexibility when sample size varies over a large range. Furthermore, AIC $c$ can be used to compare nested and non-nested models, as opposed to strictly using a Likelihood Ratio Test. 
We interpreted $\triangle \mathrm{AIC} c$ values based on the recommendations of Burnham and Anderson (1998), where $\triangle \mathrm{AIC} c<2$ between two models suggests no evidence that one of the models is best; $2<\Delta \mathrm{AIC} c<$ 4 suggests weak evidence; $4<\Delta \mathrm{AIC} c<7$ suggests some evidence; $7<\Delta \mathrm{AIC} c<10$ suggests strong evidence; and $\triangle \mathrm{AIC} c>10$ suggests very strong evidence that the model with the smaller AIC $c$ is the best model. Many of our model runs resulted in models with $\triangle \mathrm{AIC} c<2$. In these cases, models were nested and the best model could be determined by examining the deviance or likelihood and then testing using a likelihood ratio test. If the additional parameters explained a significant amount of variation then they were included in the best model.

We took a hierarchical approach to model selection by first evaluating detection parameters and determining the best model using AIC similar to the strategy of Lebreton and others (1992). This best model for detection probabilities was used for all other analyses when examining survival. We began our model selection at the farthest downstream parameter, $\lambda$, and worked our way upriver (that is, starting with $\lambda$ and then $p$ ). Next, we considered the possibility that detection probabilities could be related to total discharge so this was tested because this type of relationship could confound our results. We then assessed survival of juvenile salmonids as a function of covariates. Various combinations of variables were created for each model representing potential drivers, or a priori hypotheses, as to what influences survival of juvenile salmonids as they migrate past McNary Dam. Although hypotheses were developed a priori, selection procedures were used conceptually so that important hypotheses were not left out of the model set, which could occur without a systematic approach to hypotheses development (Collett, 2003). The effect of "study year" was then added to the best models to assess additional variation not explained by covariates. A TSW variable also was used to represent when the TSWs were operated over the multiple years of the analysis. Furthermore, environmental variables that were highly correlated (for example, fish weight vs. tag burden, total discharge vs. head) were substituted into the best models with their correlate to assess which variable was more important or had more influence regarding model fit, but were not kept within the same model if they were highly correlated or had high variance inflation factors.

In addition to constructing CJS models along with their covariates, a model was utilized for estimating survival at the weekly and yearly (that is, migration period) temporal scale. This allowed us to compare how well our covariate estimates of survival predicted survival at multiple temporal scales and provided additional temporal information for survival.

Assumptions are made when using mark-recapture models. For CJS models, these assumptions relate to inferences to the population of interest, error in interpreting acoustic signals, and statistical fit of the data to the structure of the model. The assumptions are:

1. Tagged individuals are representative of the population of interest.

2. Survival probabilities of tagged fish are the same as that of untagged fish. For example, the tagging procedures or detection of fish at downstream telemetry arrays should not influence survival or detection probabilities. If the tag negatively affects survival, then estimates of survival rates will be biased accordingly. In this study, tag weight and tag burden were examined as covariates.

3. All sampling events are instantaneous. That is, sampling should take place over a short distance relative to the distance between telemetry arrays so that the chance of mortality at a telemetry array is minimized. This assumption is necessary to attribute mortality correctly to a specific river reach. This assumption usually is satisfied by the location of telemetry arrays.

4. The fate of each tagged fish is independent of the fate of other tagged fish. Therefore, survival or mortality of one fish has no effect on the survival or mortality of the other fish. 
5. The prior detection history of a tagged fish has no effect on its subsequent survival. This assumption could be violated if parts of the river are not monitored for tagged fish. For acoustic telemetry, this assumption usually is satisfied by the passive nature of detecting acoustic tags, by monitoring all routes of passage at a dam, and by monitoring the entire cross-section of the river channel.

6. All tagged fish alive at a sampling location have the same detection probability. This assumption also could be violated as described in assumption 5, but usually is satisfied with acoustic telemetry by monitoring the entire cross section of the river channel.

7. All tags are identified correctly and the status of tagged fish (that is, alive or dead) is known without error. This assumes that fish do not lose their tags and that the tag is functioning when the fish is in the study area. Additionally, the assumption is that all detections are of live fish and that dead fish are not detected and interpreted as live (that is, false-positive detections). This assumption is addressed by releasing tagged, dead fish and seeing if we detect them at our detection arrays used to assess survival of juvenile salmonids. Furthermore, tag life studies are performed in conjunction with field studies.

There are formal ways proposed by Burnham and others (1987) to test some of these assumptionssuch as the independence of fate of individuals, fish in a group having equal survival and detection probabilities, and prior recapture history not influencing survival and detection probabilities downstream. However, these tests require three downstream detection sites which we did not have during all study years. Nonetheless, others have found that survival estimates generated with CJS models are robust to many violations of these assumptions (Skalski and others, 1998). Tag life studies and the release of tagged, dead fish were conducted during field studies (Adams and others, 1998, 2008; Perry and others, 2006, 2007; Adams and Counihan, 2009; Adams and Liedtke, 2010).

\section{Results}

\section{Yearling Chinook Salmon}

\section{Passage Analysis}

The best model explaining passage probabilities for yearling Chinook salmon at McNary Dam included covariates for photoperiod, percent spill, total discharge, and the interaction between percent spill and total discharge for all routes, as well as fish weight for fish passing through the bypass (table 3). As percent spill increased, more fish passed the dam through the spillway, and less passed through both turbines and the juvenile bypass system (figs. 6 and 7). As total discharge increased, more fish passed through the spillway and less passed through turbines. With increased discharge, more fish also passed through the bypass system when percent spill was low, and more fish passed through turbines during low discharge and low percent spill conditions (figs. 6 and 7). Furthermore, passage through both the spillway and bypass were higher during the day. Fish weight was significant for fish passing through the juvenile bypass system and showed that smaller fish had a higher probability of passing the dam through the juvenile bypass system (tables 3 and 4 , fig. 8). Temperature was not significant in explaining passage probabilities for all routes. A factor for whether the TSWs were installed explained a significant amount of variation for passage and was positively $\left(\beta_{\mathrm{TSW}}=0.218\right)$ related to passage through the spillway only when a year factor was not included. 
Table 3. Passage models for yearling Chinook salmon that passed through McNary Dam, 2004-09, comparing the full model with models that have one variable removed.

(NLL, negative log likelihood; Df, degrees of freedom; $\chi^{2}$, chi-square statistic for likelihood ratio test).

\begin{tabular}{|c|c|c|c|c|c|c|}
\hline Model & $\begin{array}{l}\text { Number of } \\
\text { parameters }\end{array}$ & $\mathrm{AIC} c$ & NLL & $\chi^{2}$ & Df & $p$ \\
\hline \multicolumn{7}{|c|}{ Spillway } \\
\hline Full (all variables) & 14 & $18,793.95$ & $9,382.98$ & NA & NA & NA \\
\hline - Photoperiod & 13 & $18,867.92$ & $9,420.96$ & 75.97 & 1 & 0 \\
\hline - \%Spill & 13 & $18,860.16$ & $9,417.08$ & 68.21 & 1 & 0 \\
\hline - Discharge & 13 & $18,872.72$ & $9,423.36$ & 80.76 & 1 & 0 \\
\hline - Weight & 13 & $18,795.37$ & $9,384.68$ & 3.42 & 1 & 0.0644 \\
\hline - Temperature & 13 & $18,791.97$ & $9,382.99$ & 0.02 & 1 & 0.8875 \\
\hline - \%Spill $\times$ Discharge & 13 & $18,799.75$ & $9,386.87$ & 7.8 & 1 & 0.0052 \\
\hline \multicolumn{7}{|c|}{ Bypass } \\
\hline - Photoperiod & 13 & $18,817.78$ & $9,395.89$ & 25.83 & 1 & 0 \\
\hline - \%Spill & 13 & $18,811.6$ & $9,392.8$ & 19.65 & 1 & 0 \\
\hline - Discharge & 13 & $18,810.77$ & $9,392.39$ & 18.82 & 1 & 0 \\
\hline - Weight & 13 & $18,798.85$ & $9,386.43$ & 6.9 & 1 & 0.0086 \\
\hline - Temperature & 13 & $18,793.84$ & $9,383.92$ & 1.89 & 1 & 0.1692 \\
\hline$-\%$ Spill $\times$ Discharge & 13 & $18,835.65$ & $9,404.83$ & 43.7 & 1 & 0 \\
\hline
\end{tabular}




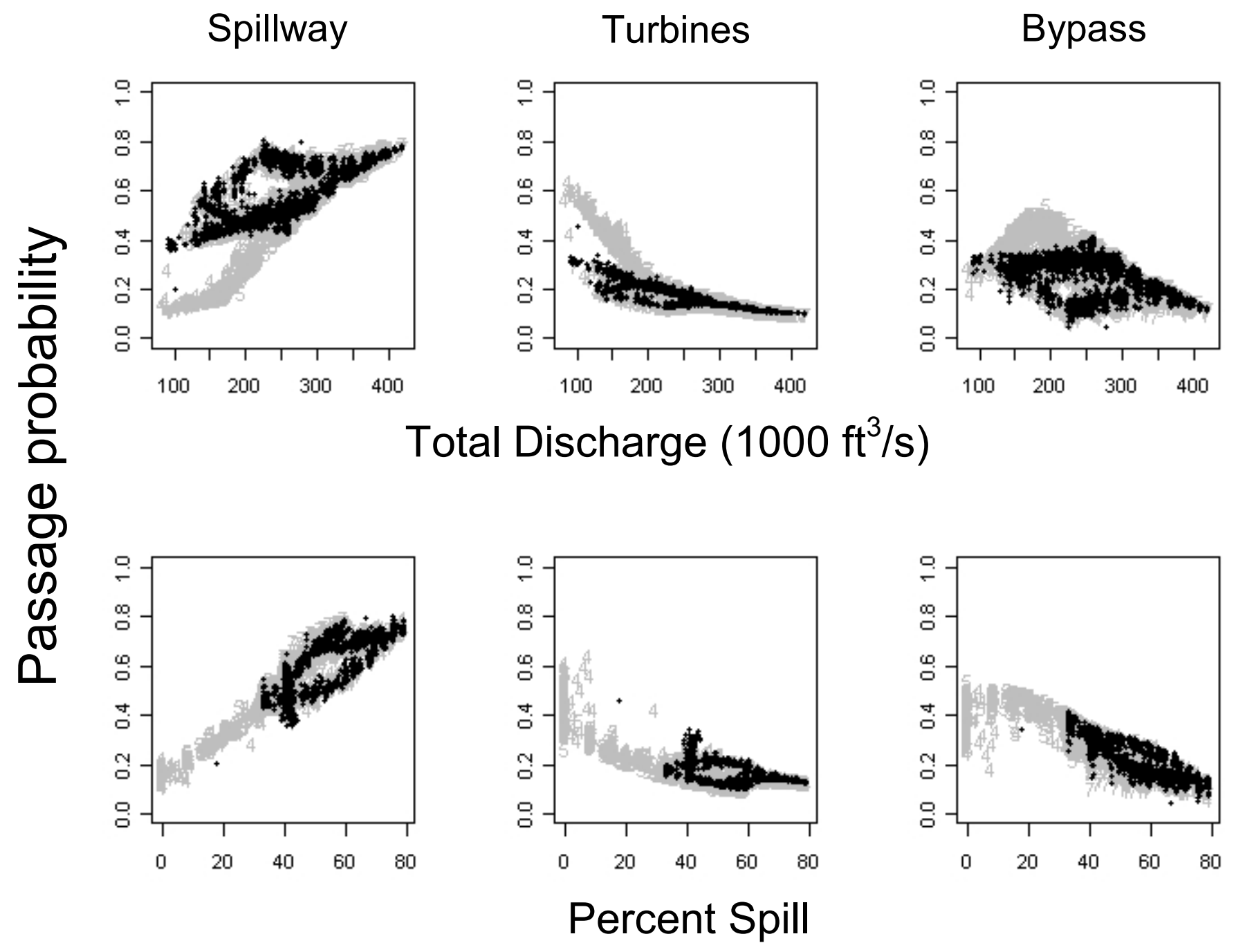

Figure 6. Passage probabilities of yearling Chinook salmon predicted by continuous covariates at McNary Dam through the spillway, turbines, and the juvenile bypass system in relation to total discharge and percent spill during day (grey symbols) and night (black symbols) photoperiods, 2004-09. Numbers used as plot symbols represent year of study, for example, 4=2004). 
Spillway

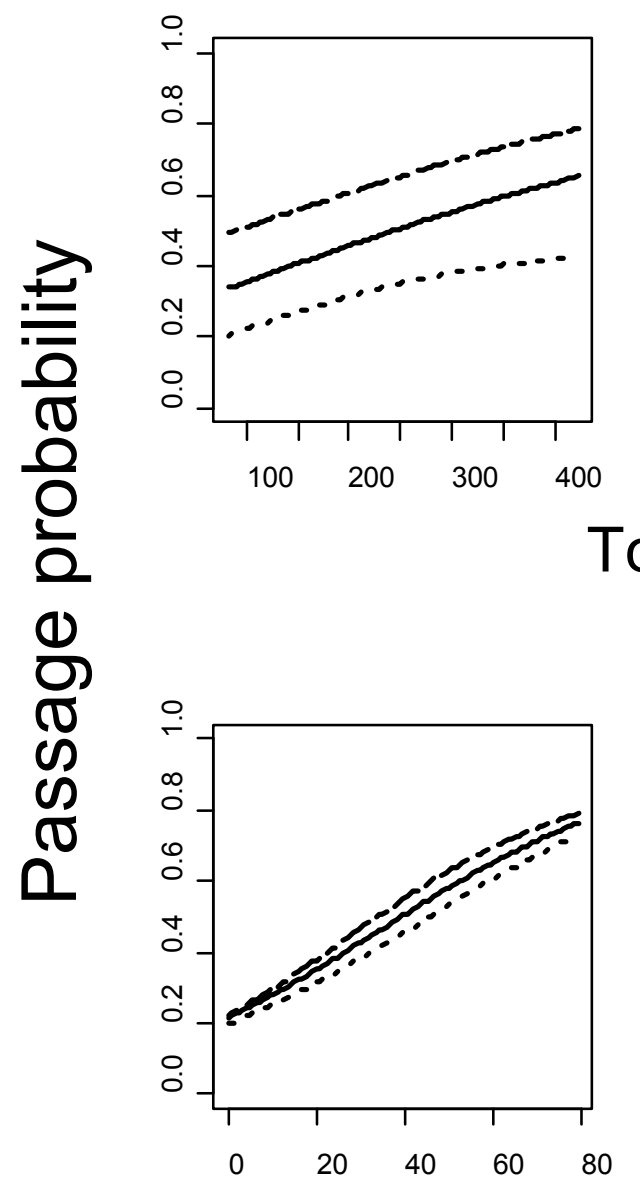

Turbines

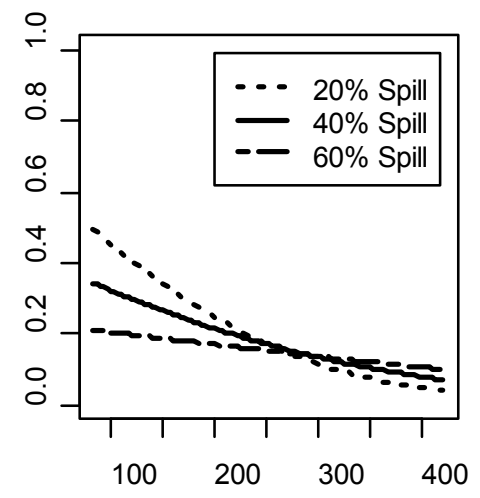

Total Discharge $\left(1000 \mathrm{ft}^{3} / \mathrm{s}\right)$
Bypass

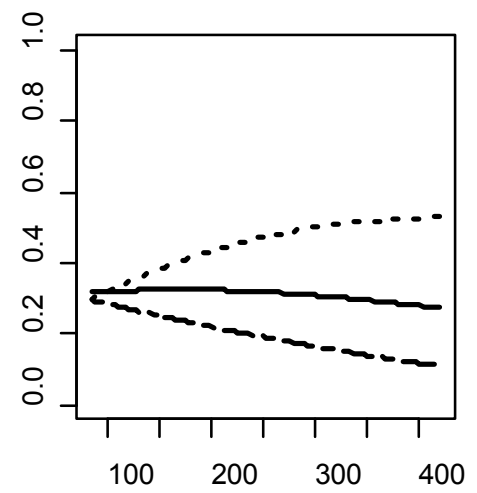

$\begin{array}{llll}100 & 200 & 300 & 400\end{array}$

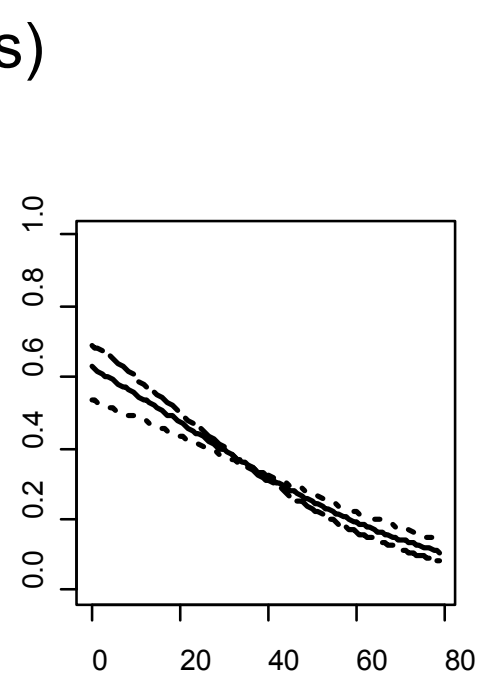

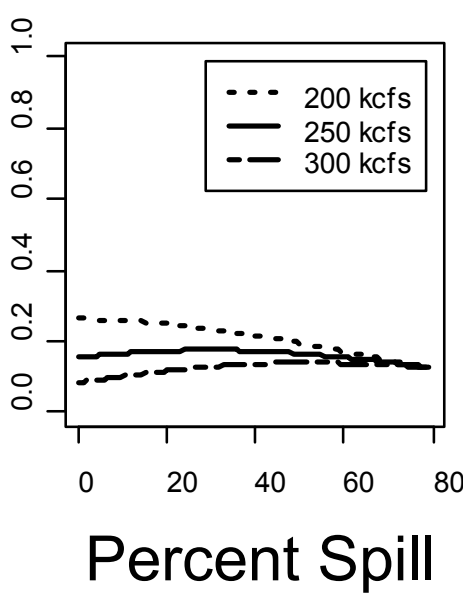




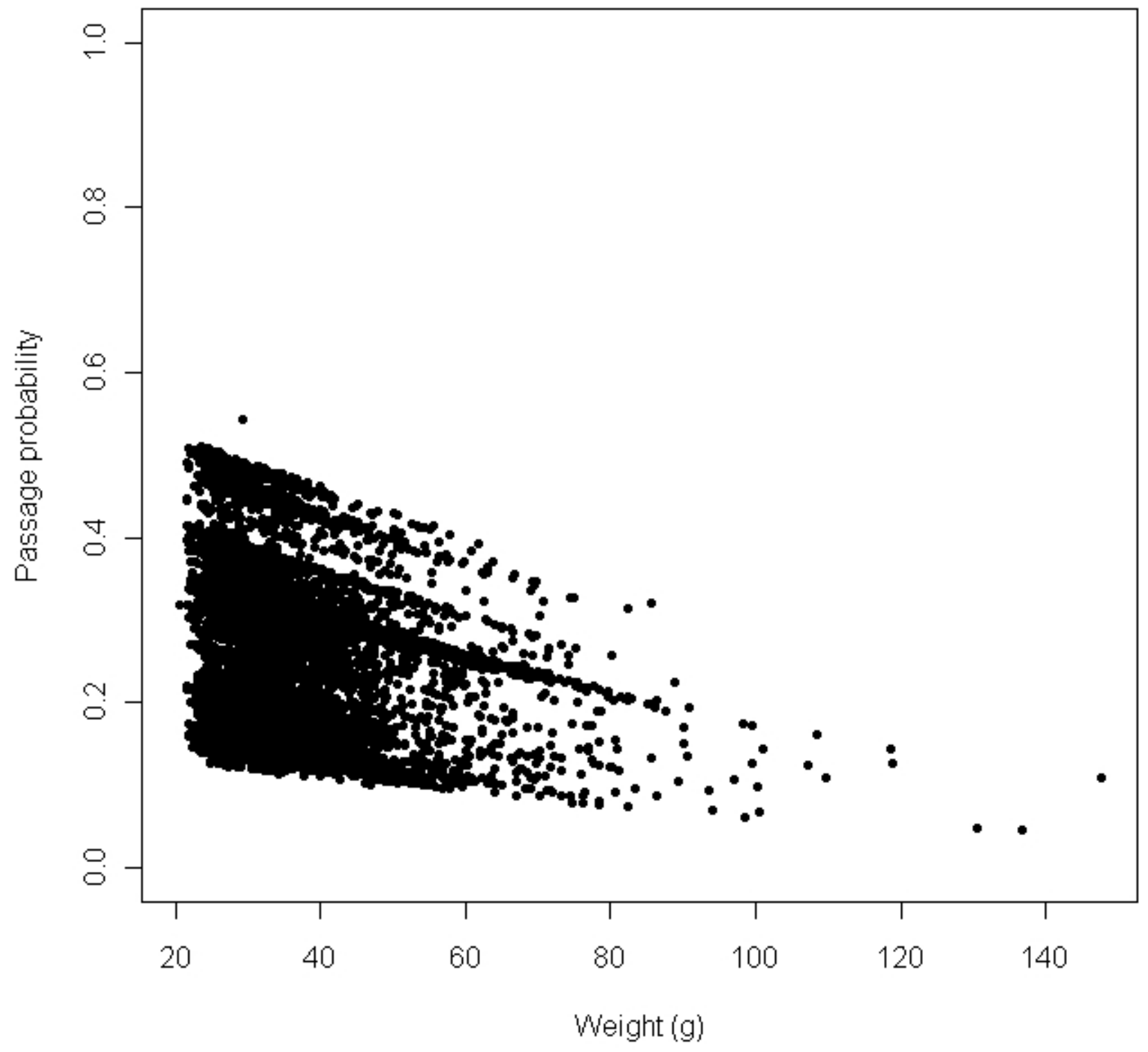

Figure 8. Passage probabilities of yearling Chinook salmon predicted by continuous covariates at McNary Dam through the juvenile bypass system in relation to fish weight, 2004-09. 
Table 4. Slope (Beta) coefficients, standard error, and 95-percent confidence limits of model parameters of passage probabilities for yearling Chinook salmon passing McNary Dam, 2004-09.

[CL, confidence limit]

\begin{tabular}{lcccc}
\hline \multicolumn{1}{c}{ Variable } & \multicolumn{1}{c}{ Beta } & $\begin{array}{c}\text { Standard } \\
\text { error }\end{array}$ & Lower CL & Upper CL \\
\hline \multicolumn{4}{c}{ Spillway } \\
& 0.788 & 0.055 & 0.680 & 0.896 \\
\hline Photoperiod (dark) & 0.581 & 0.066 & 0.452 & 0.709 \\
Photoperiod (light) & 0.414 & 0.050 & 0.315 & 0.513 \\
\%Spill & 0.353 & 0.039 & 0.276 & 0.430 \\
Discharge & -0.131 & 0.048 & -0.226 & -0.036 \\
Discharge×\%Spill & Bypass & & & \\
\hline & 0.295 & 0.061 & 0.176 & 0.415 \\
\hline Photoperiod (dark) & 0.383 & 0.073 & 0.240 & 0.527 \\
Photoperiod (light) & -0.128 & 0.024 & -0.175 & -0.080 \\
Weight & -0.250 & 0.058 & -0.363 & -0.138 \\
\%Spill & 0.179 & 0.038 & 0.104 & 0.253 \\
Discharge & -0.233 & 0.036 & -0.303 & -0.163 \\
Discharge $\times$ Spill & & & & \\
\hline
\end{tabular}




\section{Survival Analysis}

For the majority of the analyses of yearling Chinook salmon survival, the best model for $p$ and $\lambda$ was the model that expressed both parameters with a year effect (table 5). The exception was for survival through the turbines where the best model expressed $\lambda$ constant but $p$ separate across years.

Table 5. Model selection results based on varying $\lambda$ and $p$ with respect to year for yearling Chinook salmon, 2004-09.

[The best-fit models are indicated in bold. The best-fit model from assessing $\lambda$ was then used to assess the best-fit model for $p$. $\varnothing$, the probability of survival in the forebay or from dam passage through a route to the first downstream detection array; $p$, the probability of being detected at the first downstream detection array given the individual survived; $\lambda$, the joint probability of surviving and being detected from the first downstream array to the next downstream array; $y$, year]

\begin{tabular}{|c|c|c|c|c|c|}
\hline Number & Model & $\begin{array}{c}\text { Number of } \\
\text { parameters }\end{array}$ & $\mathrm{AIC} c$ & $\Delta \mathrm{AIC} c$ & Deviance \\
\hline \multicolumn{6}{|c|}{ Forebay } \\
\hline 1 & $\theta(\mathrm{y}) p(\mathrm{y}) \lambda(\mathrm{y})$ & 15 & $5,845.51$ & 0.00 & 0.000 \\
\hline 2 & $\varnothing(\mathrm{y}) p(\mathrm{y}) \lambda$ & 11 & $5,915.12$ & 69.61 & 77.628 \\
\hline 3 & $\varnothing(\mathrm{y}) p \lambda(\mathrm{y})$ & 11 & $5,946.40$ & 100.89 & 108.910 \\
\hline \multicolumn{6}{|c|}{ Turbine } \\
\hline 2 & $\boldsymbol{\theta}(\mathrm{y}) p(\mathrm{y}) \lambda$ & 13 & $2,625.05$ & 0.00 & 4.803 \\
\hline 1 & $\varnothing(\mathrm{y}) p(\mathrm{y}) \lambda(\mathrm{y})$ & 18 & $2,630.40$ & 5.35 & 0.000 \\
\hline 3 & $\varnothing(\mathrm{y}) p \lambda$ & 8 & $2,839.62$ & 214.57 & 229.482 \\
\hline \multicolumn{6}{|c|}{ Bypass } \\
\hline 1 & $\theta(\mathrm{y}) p(\mathrm{y}) \lambda(\mathrm{y})$ & 18 & $5,535.64$ & 0.00 & 0.000 \\
\hline 2 & $\varnothing(\mathrm{y}) p(\mathrm{y}) \lambda$ & 13 & $5,544.39$ & 8.76 & 18.822 \\
\hline 3 & $\varnothing(\mathrm{y}) p \lambda(\mathrm{y})$ & 13 & $6,140.59$ & 604.96 & 615.023 \\
\hline \multicolumn{6}{|c|}{ Spillway } \\
\hline 1 & $\theta(\mathrm{y}) p(\mathrm{y}) \lambda(\mathrm{y})$ & 18 & $10,040.63$ & 0.00 & 0.000 \\
\hline 2 & $\varnothing(\mathrm{y}) p(\mathrm{y}) \lambda$ & 13 & $10,069.04$ & 28.42 & 38.452 \\
\hline 3 & $\varnothing(\mathrm{y}) p \lambda(\mathrm{y})$ & 13 & $10,968.23$ & 927.60 & 937.636 \\
\hline \multicolumn{6}{|c|}{ Tailrace Releases } \\
\hline 1 & $\theta(\mathrm{y}) p(\mathrm{y}) \lambda(\mathrm{y})$ & 18 & $11,390.94$ & 0.00 & 0.000 \\
\hline 2 & $\varnothing(\mathrm{y}) p(\mathrm{y}) \lambda$ & 13 & $11,431.49$ & 40.55 & 50.582 \\
\hline 3 & $\varnothing(\mathrm{y}) p \lambda(\mathrm{y})$ & 13 & $12,923.52$ & $1,532.59$ & $1,542.617$ \\
\hline
\end{tabular}


Forebay survival was most related to fish weight, water temperature, and total discharge (table 6). Water temperature was negatively related to forebay survival, resulting in estimates that ranged from 0.948 to 0.999 for the temperatures observed (fig. 9, table 7). Survival of fish in the forebay was positively related to fish weight and discharge (fig. 9, table 7). Survival predicted by discharge ranged from 0.929 to 0.999 , whereas, survival predicted by fish weight ranged from 0.986 to 0.999 . Tag burden was substituted into the models to determine how tag burden and fish weight affected the fit of the model. We found that the best model supported fish weight by greater than 5 AIC units.

The best-fit model for survival of fish passing through the turbines included photoperiod, fish weight, and water temperature (table 8). The addition of passage location contributed nothing to model fit. Survival for yearling Chinook salmon passing through the turbines was positively related to fish weight, negatively related to temperature, and was lower during the day photoperiod (table 7, fig. 10). Photoperiod explained a significant amount of variation in survival. The models that did not include group covariates for photoperiod had AICc values 10 units higher than the models that included covariates for photoperiod (table 8). Because fish weight and tag burden were highly correlated $(\mathrm{r}=$ 0.850), tag burden was examined and a negative relationship was observed to survival (table 7, fig. 10). The model that included tag burden had an AIC value more than two units lower than the model that included fish weight, which indicated weak evidence for choosing one model over the other (table 8).

For yearling Chinook salmon that passed McNary Dam through the juvenile bypass system, the best model included only group covariates for photoperiod (table 9). There were other models within two AIC units, but these did not contribute significantly to model fit. Survival of fish passing through the bypass system was higher during the night (0.962) than the day (0.933) photoperiod by about 3 percent. The model that included photoperiod explained a significant amount of variation and fit better than the model without photoperiod, having a difference of greater than four AIC units.

For yearling Chinook salmon that passed McNary Dam through the spillway, the best model included spillway passage location, fish weight, and total discharge (table 10). Survival was positively related to both discharge and fish weight (fig. 11). Passage location was an important variable describing variation in survival only because of the 'Unknown' category for fish that could not be assigned to a particular section of the spillway. We were able to determine that these fish passed the spillway, but we could not definitively assign them to the north or south passage areas. This group of 'Unknown' fish had lower survival than fish that were assigned as either passing the north or south portion of the spillway. Survival estimates between fish that passed the north versus the south spillway were similar. Fish weight and tag burden were highly correlated $(r=-0.863)$ so tag burden was examined and a negative relation was observed to survival (fig. 11). The model that included tag burden was within two AIC units of the model that included fish weight, which indicated that both models were supported equally.

Fish released in the tailrace were not significantly related to the covariates we examined (table 11). Furthermore, release location also was tested to determine if there was a difference in survival among fish released in the north, middle, or south portion of the channel in the tailrace and there was no statistical difference.

A year factor was added to the best model for survival of yearling Chinook salmon for each route or reach to determine if it would explain any additional variation in survival. Adding a year effect only explained additional variation for fish migrating through the forebay. Furthermore, a factor indicating whether a TSW was installed during a particular year also was included in place of year when examining survival through the spillway and survival in the forebay. This factor was not significant for survival through the spillway, but was significant for survival of fish migrating through the forebay and was about 0.8 percent higher during years where TSWs were installed (fig. 9). 

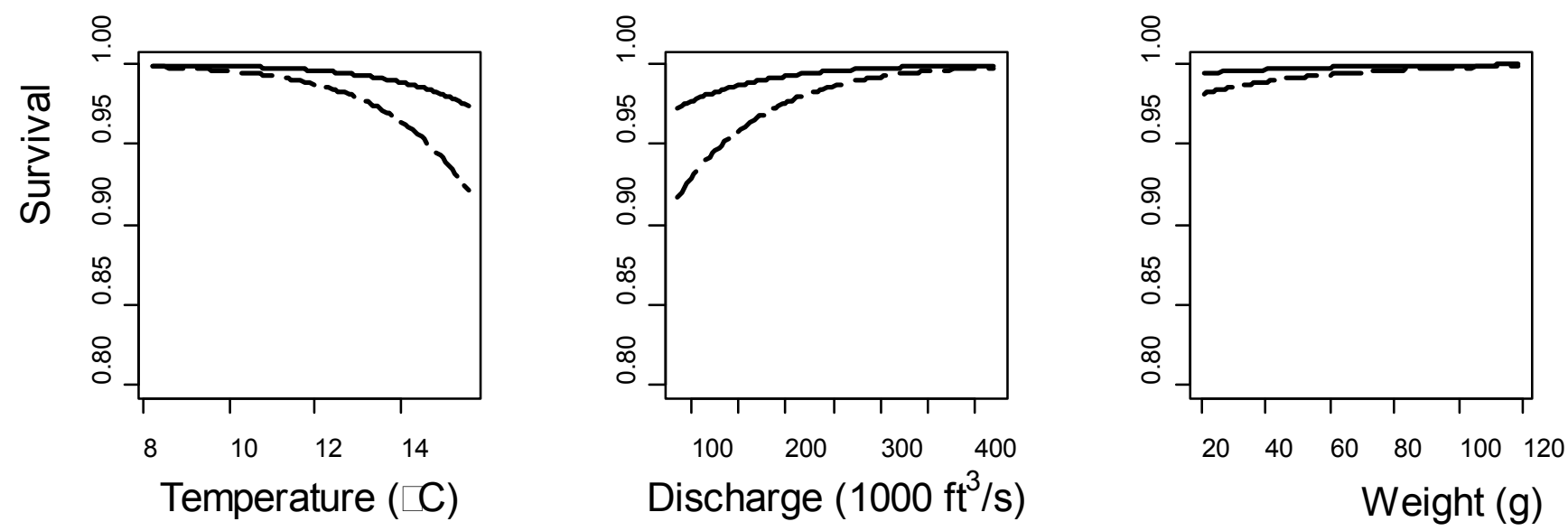

Figure 9. Survival of yearling Chinook salmon migrating through the forebay of McNary Dam when temporary spillway weirs were not installed (solid line, 2005 and 2006) and were installed (dashed line, 2007-09) in relation to water temperature, total discharge, and fish weight. Other covariates in the model were held constant at their mean value to examine the relationship to the variable of interest. 
Table 6. Model selection for fish that passed through the forebay at McNary Dam relating survival $(\varnothing)$ as a function of environmental variables using the fixed structure of the best model for $p$ and $\lambda$ parameters for yearling Chinook salmon, 2005-09.

[The best-fit model is indicated in bold]

\begin{tabular}{|c|c|c|c|c|c|}
\hline Model & $\begin{array}{c}\text { Number of } \\
\text { parameters }\end{array}$ & $\mathrm{AIC} c$ & $\begin{array}{l}\Delta \mathrm{AIC} c \\
\text { (group) }\end{array}$ & $\begin{array}{c}\Delta \mathrm{AIC} c \\
\text { (overall) }\end{array}$ & Deviance \\
\hline Intercept only & 11 & $5,908.80$ & 92.58 & 92.58 & 90.076 \\
\hline Temperature & 12 & $5,868.13$ & 51.91 & 51.91 & $5,844.110$ \\
\hline Discharge & 12 & $5,886.24$ & 70.02 & 70.02 & $5,862.213$ \\
\hline$\%$ Spill & 12 & $5,896.48$ & 80.25 & 80.25 & $5,872.452$ \\
\hline Weight & 12 & $5,901.39$ & 85.17 & 85.17 & $5,877.365$ \\
\hline Temperature + Discharge & 13 & $5,820.09$ & 3.87 & 3.87 & $5,794.063$ \\
\hline Temperature $+\%$ Spill & 13 & $5,853.03$ & 36.81 & 36.81 & $5,827.005$ \\
\hline$\%$ Spill + Discharge & 13 & $5,885.84$ & 69.62 & 69.62 & $5,859.811$ \\
\hline Weight + Temperature + Discharge & 14 & $5,816.22$ & 0.00 & 0.00 & $5,788.189$ \\
\hline Temperature + Discharge $+\%$ Spill & 14 & $5,822.06$ & 5.84 & 5.84 & $5,794.031$ \\
\hline Weight + Temperature $+\%$ Spill & 14 & $5,851.39$ & 35.17 & 35.17 & $5,823.361$ \\
\hline Weight $+\%$ Spill + Discharge & 14 & $5,873.75$ & 57.53 & 57.53 & $5,845.714$ \\
\hline$\%$ Spill + Discharge $+\%$ Spill $\times$ Discharge & 14 & $5,887.44$ & 71.22 & 71.22 & $5,859.413$ \\
\hline Weight + Temperature + Discharge $+\%$ Spill & 15 & $5,818.13$ & 1.91 & 1.91 & $5,788.094$ \\
\hline Temperature + Discharge $+\%$ Spill $+\%$ Spill $\times$ Discharge & 15 & $5,823.58$ & 7.36 & 7.36 & $5,793.545$ \\
\hline Weight $+\%$ Spill + Discharge $+\%$ Spill $\times$ Discharge & 15 & $5,875.02$ & 58.80 & 58.80 & $5,844.985$ \\
\hline Weight + Temperature + Discharge $+\%$ Spill $+\%$ Spill $\times$ Discharge & 16 & $5,819.42$ & 3.20 & 3.20 & $5,787.376$ \\
\hline
\end{tabular}


Table 6 (continued). Model selection for fish that passed through the forebay at McNary Dam relating survival (ø) as a function of environmental variables and group covariates for photoperiod using the fixed structure of the best model for $p$ and $\lambda$ parameters for yearling Chinook salmon, 2005-09.

\begin{tabular}{|c|c|c|c|c|c|}
\hline Model & $\begin{array}{l}\text { Number of } \\
\text { parameters }\end{array}$ & $\mathrm{AlC}_{c}$ & $\begin{array}{l}\Delta \mathrm{AIC} c \\
\text { (group) }\end{array}$ & $\begin{array}{c}\Delta \mathrm{AIC} c \\
\text { (overall) }\end{array}$ & Deviance \\
\hline Photoperiod + Discharge & 13 & $5,886.76$ & $6 \quad 67.28$ & 70.54 & $5,860.736$ \\
\hline Photoperiod $+\%$ Spill & 13 & $5,897.74$ & $4 \quad 78.25$ & 81.52 & $5,871.710$ \\
\hline Photoperiod + Temperature & 13 & $5,866.96$ & $6 \quad 50.74$ & 50.74 & $5,840.929$ \\
\hline Photoperiod + Weight & 13 & $5,899.85$ & $5 \quad 80.37$ & 83.63 & $5,873.827$ \\
\hline Photoperiod + Weight + Discharge & 14 & $5,873.87$ & $7 \quad 54.38$ & 57.65 & $5,845.836$ \\
\hline Photoperiod + Weight + Temperature & 14 & $5,864.62$ & 248.40 & 48.40 & $5,836.587$ \\
\hline Photoperiod $+\%$ Spill + Discharge & 14 & $5,887.27$ & $7 \quad 67.78$ & 71.05 & $5,859.235$ \\
\hline Photoperiod + Weight $+\%$ Spill & 14 & $5,890.31$ & 170.82 & 74.09 & $5,862.279$ \\
\hline Photoperiod $+\%$ Spill + Discharge + Temperature & 15 & $5,823.44$ & 3.96 & 7.22 & $5,793.408$ \\
\hline Photoperiod + Weight $+\%$ Spill + Discharge & 15 & $5,875.09$ & 955.61 & 58.87 & $5,845.058$ \\
\hline Photoperiod $+\%$ Spill + Discharge $+\%$ Spill $\times$ Discharge & 15 & $5,888.95$ & $5 \quad 69.46$ & 72.73 & $5,858.914$ \\
\hline Photoperiod + Weight $+\%$ Spill + Discharge + Temperature & 16 & $5,819.49$ & 0.00 & 3.27 & $5,787.446$ \\
\hline Photoperiod $+\%$ Spill + Discharge + Temperature $+\%$ Spill $\times$ Discharge & 16 & $5,825.07$ & 5.58 & 8.85 & $5,793.030$ \\
\hline Photoperiod + Weight $+\%$ Spill + Discharge $+\%$ Spill $\times$ Discharge & 16 & $5,876.49$ & 957.01 & 60.27 & $5,844.454$ \\
\hline Photoperiod + Weight $+\%$ Spill + Discharge + Temperature $+\%$ Spill $\times$ Discharge & 17 & $5,820.91$ & 1.43 & 4.69 & $5,786.867$ \\
\hline
\end{tabular}


Table 7. Slope (Beta) coefficients, standard error, and 95-percent confidence limits of model parameters for yearling Chinook salmon passing McNary Dam, 2004-09.

[CL, confidence limit]

\begin{tabular}{|c|c|c|c|c|}
\hline Variable & Beta & $\begin{array}{l}\text { Standard } \\
\text { error }\end{array}$ & Lower CL & Upper CL \\
\hline \multicolumn{5}{|c|}{ Forebay } \\
\hline Intercept (TSW not installed) & 6.907 & 1.128 & 4.697 & 9.118 \\
\hline TSW (installed) & 1.170 & 0.285 & 0.612 & 1.728 \\
\hline Weight & 0.027 & 0.015 & -0.002 & 0.056 \\
\hline Temperature & -0.533 & 0.077 & -0.685 & -0.382 \\
\hline Discharge & 0.011 & 0.002 & 0.008 & 0.015 \\
\hline \multicolumn{5}{|c|}{ Turbine } \\
\hline Intercept & 4.732 & 0.658 & 3.443 & 6.021 \\
\hline Temperature & -0.114 & 0.048 & -0.207 & -0.020 \\
\hline Photoperiod (light) & -0.643 & 0.200 & -1.034 & -0.251 \\
\hline Tag Burden & -0.273 & 0.079 & -0.428 & -0.117 \\
\hline \multicolumn{5}{|c|}{ Bypass } \\
\hline Intercept & 3.236 & 0.266 & 2.714 & 3.757 \\
\hline Photoperiod (light) & -0.598 & 0.282 & -1.150 & -0.046 \\
\hline \multicolumn{5}{|c|}{ Spillway } \\
\hline Intercept & 0.653 & 0.530 & -0.385 & 1.692 \\
\hline SpillPassLoc (north) & 0.925 & 0.289 & 0.358 & 1.493 \\
\hline SpillPassLoc (south) & 0.689 & 0.289 & 0.122 & 1.255 \\
\hline Weight & 0.024 & 0.009 & 0.006 & 0.042 \\
\hline Discharge & 0.002 & 0.001 & 0.0001 & 0.005 \\
\hline \multicolumn{5}{|c|}{ Tailrace Releases } \\
\hline Intercept & 3.695 & 0.111 & 3.478 & 3.913 \\
\hline
\end{tabular}


Table 8. Model selection for fish that passed through turbines at McNary Dam relating survival (ø) as a function of environmental variables using the fixed structure of the best model for $p$ and $\lambda$ parameters for yearling Chinook salmon, 2004-09.

\begin{tabular}{|c|c|c|c|c|c|}
\hline Model & $\begin{array}{l}\text { Number of } \\
\text { parameters }\end{array}$ & $\mathrm{AIC} c$ & $\begin{array}{l}\Delta \mathrm{AIC} c \\
\text { (group) }\end{array}$ & $\begin{array}{c}\Delta \mathrm{AIC} c \\
\text { (overall) }\end{array}$ & Deviance \\
\hline Intercept only & 8 & $2,626.34$ & 14.03 & 24.34 & 90.481 \\
\hline Weight & 9 & $2,616.67$ & 4.36 & 14.67 & $2,598.580$ \\
\hline Temperature & 9 & $2,618.42$ & 6.11 & 16.42 & $2,600.333$ \\
\hline Discharge & 9 & $2,627.29$ & 14.99 & 25.30 & $2,609.208$ \\
\hline$\%$ Spill & 9 & $2,628.33$ & 16.02 & 26.33 & $2,610.243$ \\
\hline Temperature + Discharge & 10 & $2,620.43$ & 8.12 & 18.44 & $2,600.324$ \\
\hline Temperature $+\%$ Spill & 10 & $2,620.43$ & 8.12 & 18.44 & $2,600.325$ \\
\hline Weight + Temperature & 10 & $2,612.31$ & 0.00 & 10.31 & $2,592.201$ \\
\hline$\%$ Spill + Discharge & 10 & $2,628.44$ & 16.13 & 26.44 & $2,608.331$ \\
\hline$\%$ Spill + Discharge $+\%$ Spill $\times$ Discharge & 11 & $2,628.26$ & 15.95 & 26.26 & $2,606.132$ \\
\hline Weight + Temperature $+\%$ Spill & 11 & $2,614.29$ & 1.99 & 12.30 & $2,592.168$ \\
\hline Weight + Temperature + Discharge & 11 & $2,614.32$ & 2.01 & 12.32 & $2,592.192$ \\
\hline Weight $+\%$ Spill + Discharge & 11 & $2,619.76$ & 7.45 & 17.76 & $2,597.633$ \\
\hline Temperature + Discharge $+\%$ Spill & 11 & $2,622.42$ & 10.11 & 20.42 & $2,600.289$ \\
\hline Weight + Temperature + Discharge $+\%$ Spill & 12 & $2,616.32$ & 4.01 & 14.32 & $2,592.167$ \\
\hline Weight $+\%$ Spill + Discharge $+\%$ Spill $\times$ Discharge & 12 & $2,620.52$ & 8.21 & 18.52 & $2,596.368$ \\
\hline Temperature + Discharge $+\%$ Spill $+\%$ Spill $\times$ Discharge & 12 & $2,623.37$ & 11.07 & 21.38 & $2,599.223$ \\
\hline Weight + Temperature + Discharge $+\%$ Spill $+\%$ Spill $\times$ Discharge & 13 & $2,617.69$ & 5.38 & 15.69 & $2,591.512$ \\
\hline
\end{tabular}


Table 8 (continued). Model selection for fish that passed through turbines at McNary Dam relating survival $(\boldsymbol{\theta})$ as a function of environmental variables and group covariates for photoperiod using the fixed structure of the best model for $\boldsymbol{p}$ and $\lambda$ parameters for yearling Chinook salmon, 2004-09.

[The best-fit model is indicated in bold]

\begin{tabular}{|c|c|c|c|c|c|}
\hline Model & $\begin{array}{l}\text { Number of } \\
\text { parameters }\end{array}$ & $\mathrm{AIC} c$ & $\begin{array}{l}\Delta \mathrm{AIC} c \\
\text { (group) }\end{array}$ & $\begin{array}{c}\Delta \mathrm{AIC} c \\
\text { (overall) }\end{array}$ & Deviance \\
\hline Photoperiod & 9 & $2,617.07$ & 15.07 & 15.07 & 79.192 \\
\hline Photoperiod + Weight & 10 & $2,605.55$ & 3.55 & 3.55 & $2,585.441$ \\
\hline Photoperiod + Temperature & 10 & $2,609.52$ & 7.53 & 7.53 & $2,589.417$ \\
\hline Photoperiod + Discharge & 10 & $2,616.68$ & 14.68 & 14.68 & $2,596.570$ \\
\hline Photoperiod + \%Spill & 10 & $2,617.62$ & 15.63 & 15.63 & $2,597.519$ \\
\hline Photoperiod + Weight + Temperature & 11 & $2,602.00$ & 0.00 & 0.00 & $2,579.868$ \\
\hline Photoperiod + Weight $+\%$ Spill & 11 & $2,605.89$ & 3.89 & 3.89 & $2,583.760$ \\
\hline Photoperiod + Weight + Discharge & 11 & $2,606.07$ & 4.08 & 4.08 & $2,583.943$ \\
\hline Photoperiod $+\%$ Spill + Discharge & 11 & $2,618.60$ & 16.61 & 16.61 & $2,596.477$ \\
\hline Photoperiod + Weight $+\%$ Spill + Discharge & 12 & $2,607.65$ & 5.65 & 5.65 & $2,583.498$ \\
\hline Photoperiod $+\%$ Spill + Discharge + Temperature & 12 & $2,611.82$ & 9.82 & 9.82 & $2,587.669$ \\
\hline Photoperiod $+\%$ Spill + Discharge $+\%$ Spill $\times$ Discharge & 12 & $2,617.86$ & 15.87 & 15.87 & $2,593.712$ \\
\hline Photoperiod + Weight $+\%$ Spill + Discharge + Temperature & 13 & $2,604.20$ & 2.20 & 2.20 & $2,578.020$ \\
\hline Photoperiod + Weight + Photoperiod $+\%$ Spill + Discharge $+\%$ Spill $\times$ Discharge & 13 & $2,608.07$ & 6.07 & 6.07 & $2,581.890$ \\
\hline Photoperiod $+\%$ Spill + Discharge + Temperature $+\%$ Spill $\times$ Discharge & 13 & $2,612.29$ & 10.30 & 10.30 & $2,586.117$ \\
\hline Photoperiod + Weight $+\%$ Spill + Discharge + Temperature $+\%$ Spill $\times$ Discharge & 14 & $2,605.22$ & 3.22 & 3.22 & $2,577.013$ \\
\hline
\end{tabular}


Table 8 (continued). Model selection for fish that passed through turbines at McNary Dam relating survival $(\boldsymbol{\theta})$ as a function of environmental variables and group covariates for turbine passage location (i.e. north, south) using the fixed structure of the best model for $\boldsymbol{p}$ and $\lambda$ parameters for yearling Chinook salmon, 2004-09.

\begin{tabular}{|c|c|c|c|c|c|}
\hline Model & $\begin{array}{l}\text { Number of } \\
\text { parameters }\end{array}$ & $\mathrm{AIC}_{c}$ & $\begin{array}{l}\Delta \mathrm{AIC} c \\
\text { (group) }\end{array}$ & $\begin{array}{c}\Delta \mathrm{AIC} c \\
\text { (overall) }\end{array}$ & Deviance \\
\hline TurbPassLoc & 9 & $2,625.97$ & 13.16 & 23.97 & 88.095 \\
\hline TurbPassLoc + Weight & 10 & $2,616.41$ & 3.60 & 14.42 & $2,596.305$ \\
\hline TurbPassLoc + Discharge & 10 & $2,626.05$ & 13.24 & 24.06 & $2,605.945$ \\
\hline TurbPassLoc $+\%$ Spill & 10 & $2,627.97$ & 15.16 & 25.97 & $2,607.861$ \\
\hline TurbPassLoc + Temperature & 10 & $2,619.15$ & 6.35 & 17.16 & $2,599.047$ \\
\hline TurbPassLoc + Weight + Discharge & 11 & $2,617.37$ & 4.56 & 15.37 & $2,595.239$ \\
\hline TurbPassLoc + Weight $+\%$ Spill & 11 & $2,618.42$ & 5.62 & 16.43 & $2,596.297$ \\
\hline TurbPassLoc + Weight + Temperature & 11 & $2,612.81$ & 0.00 & 10.81 & $2,590.679$ \\
\hline TurbPassLoc + Temperature + Discharge & 11 & $2,620.98$ & 8.18 & 18.99 & $2,598.855$ \\
\hline TurbPassLoc + Temperature $+\%$ Spill & 11 & $2,621.15$ & 8.35 & 19.16 & $2,599.027$ \\
\hline TurbPassLoc + \%Spill + Discharge & 11 & $2,627.46$ & 14.65 & 25.47 & $2,605.334$ \\
\hline TurbPassLoc + Weight + Temperature + Discharge & 12 & $2,614.75$ & 1.94 & 12.75 & $2,590.595$ \\
\hline TurbPassLoc + Weight $+\%$ Spill + Discharge & 12 & $2,619.00$ & 6.19 & 17.01 & $2,594.850$ \\
\hline TurbPassLoc + Temperature + Discharge $+\%$ Spill & 12 & $2,622.98$ & 10.17 & 20.98 & $2,598.827$ \\
\hline TurbPassLoc $+\%$ Spill + Discharge $+\%$ Spill $\times$ Discharge & 12 & $2,627.79$ & 14.98 & 25.79 & $2,603.638$ \\
\hline TurbPassLoc + Weight + Temperature + Discharge $+\%$ Spill & 13 & $2,616.75$ & 3.94 & 14.75 & $2,590.573$ \\
\hline TurbPassLoc + Temperature + Discharge $+\%$ Spill $+\%$ Spill $\times$ Discharge & 13 & $2,624.15$ & 11.34 & 22.15 & $2,597.974$ \\
\hline TurbPassLoc + Weight + Temperature + Discharge $+\%$ Spill $+\%$ Spill $\times$ Discharge & 14 & $2,618.32$ & 5.51 & 16.32 & $2,590.114$ \\
\hline
\end{tabular}


Table 8 (continued). Model selection for fish that passed through turbines at McNary Dam relating survival $(\boldsymbol{\theta})$ as a function of environmental variables and group covariates for turbine passage location (i.e. north, south) and photoperiod using the fixed structure of the best model for $p$ and $\lambda$ parameters for yearling Chinook salmon, 2004-09.

\begin{tabular}{|c|c|c|c|c|c|}
\hline Model & $\begin{array}{l}\text { Number of } \\
\text { parameters }\end{array}$ & $\operatorname{AIC} c$ & $\begin{array}{l}\triangle \mathrm{AIC} c \\
\text { (group) }\end{array}$ & $\begin{array}{c}\Delta \mathrm{AIC} c \\
\text { (overall) }\end{array}$ & Deviance \\
\hline TurbPassLoc + Photoperiod & 10 & $2,616.77$ & 14.20 & 14.77 & 76.877 \\
\hline TurbPassLoc + Photoperiod + Weight & 11 & $2,605.41$ & 2.84 & 3.41 & $2,583.281$ \\
\hline TurbPassLoc + Photoperiod + Temperature & 11 & $2,610.34$ & 7.77 & 8.35 & $2,588.215$ \\
\hline TurbPassLoc + Photoperiod + Temperature & 11 & $2,610.34$ & 7.77 & 8.35 & $2,588.215$ \\
\hline TurbPassLoc + Photoperiod + Discharge & 11 & $2,614.88$ & 12.31 & 12.89 & $2,592.754$ \\
\hline TurbPassLoc + Photoperiod $+\%$ Spill & 11 & $2,616.10$ & 13.53 & 14.10 & $2,593.973$ \\
\hline TurbPassLoc + Photoperiod + Weight + Temperature & 12 & $2,602.57$ & 0.00 & 0.58 & $2,578.422$ \\
\hline TurbPassLoc + Photoperiod + Weight $+\%$ Spill & 12 & $2,604.47$ & 1.90 & 2.48 & $2,580.324$ \\
\hline TurbPassLoc + Photoperiod + Weight + Discharge & 12 & $2,604.60$ & 2.03 & 2.61 & $2,580.451$ \\
\hline TurbPassLoc + Photoperiod $+\%$ Spill + Discharge & 12 & $2,616.58$ & 14.00 & 14.58 & $2,592.425$ \\
\hline TurbPassLoc + Photoperiod + Weight $+\%$ Spill + Discharge & 13 & $2,605.86$ & 3.29 & 3.87 & $2,579.685$ \\
\hline TurbPassLoc + Photoperiod $+\%$ Spill + Discharge + Temperature & 13 & $2,611.75$ & 9.17 & 9.75 & $2,585.571$ \\
\hline TurbPassLoc + Photoperiod $+\%$ Spill + Discharge $+\%$ Spill $\times$ Discharge & 13 & $2,616.45$ & 13.88 & 14.46 & 2,590.276 \\
\hline TurbPassLoc + Photoperiod + Weight + Photoperiod $+\%$ Spill + Discharge + Temperature & 14 & $2,603.89$ & 1.31 & 1.89 & $2,575.683$ \\
\hline TurbPassLoc + Photoperiod + Weight $+\%$ Spill + Discharge $+\%$ Spill $\times$ Discharge & 14 & $2,606.78$ & 4.21 & 4.79 & $2,578.580$ \\
\hline TurbPassLoc + Photoperiod $+\%$ Spill + Discharge + Temperature $+\%$ Spill $\times$ Discharge & 14 & $2,612.52$ & 9.95 & 10.52 & $2,584.315$ \\
\hline TurbPassLoc + Photoperiod + Weight $+\%$ Spill + Discharge + Temperature $+\%$ Spill $\times$ Discharge & 15 & $2,605.19$ & 2.62 & 3.20 & $2,574.963$ \\
\hline
\end{tabular}



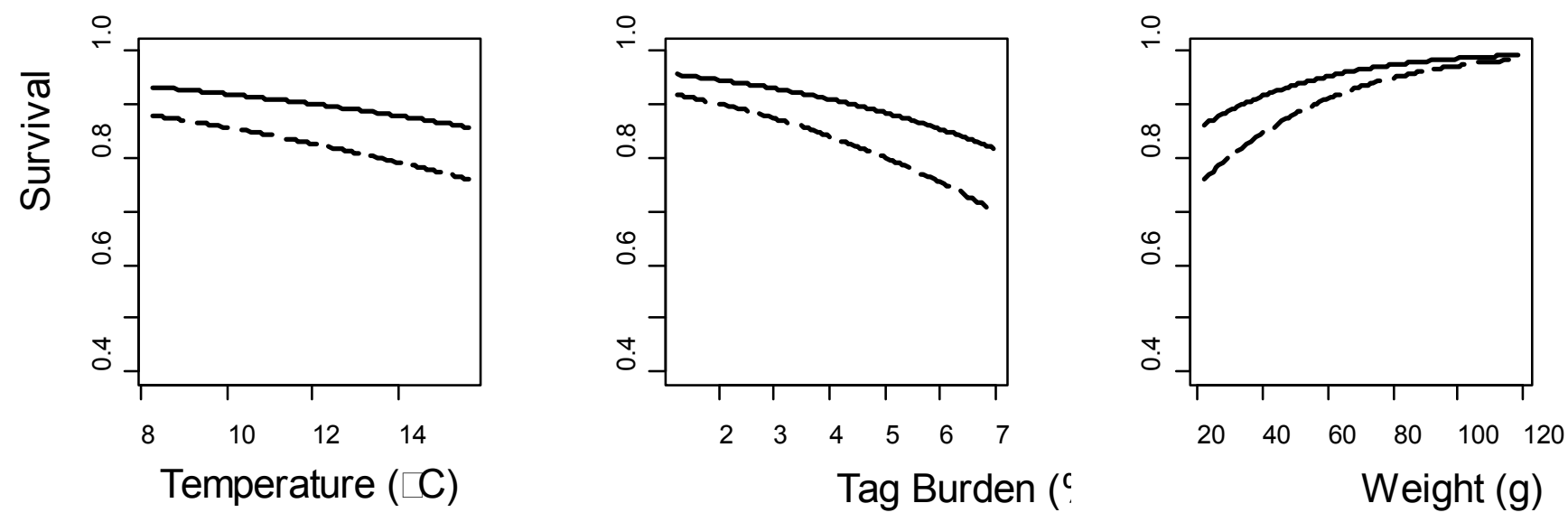

Figure 10. Survival of yearling Chinook salmon that passed through turbines at McNary Dam in relation to water temperature, tag burden (tag weightfish weight $\times 100$ ), and fish weight during day (dashed line) and night (solid line) photoperiods, 2004-09. Other covariates in the model were held constant at their mean value to examine the relationship to the variable of interest. 
Table 9. Model selection for fish that passed through the juvenile bypass system at McNary Dam relating survival (ø) as a function of environmental variables using the fixed structure of the best model for $p$ and $\lambda$ parameters for yearling Chinook salmon, 2004-09.

\begin{tabular}{|c|c|c|c|c|c|}
\hline Model & $\begin{array}{l}\text { Number of } \\
\text { parameters }\end{array}$ & $\mathrm{AlC} c$ & $\begin{array}{c}\Delta \mathrm{AIC} c \\
\text { (group) }\end{array}$ & $\begin{array}{c}\Delta \mathrm{AIC} c \\
\text { (overall) }\end{array}$ & Deviance \\
\hline Intercept only & 13 & $5,535.84$ & 2.69 & 4.96 & 33.829 \\
\hline$\%$ Spill & 14 & $5,534.96$ & 1.81 & 4.08 & $5,506.872$ \\
\hline Discharge & 14 & $5,535.30$ & 2.15 & 4.42 & $5,507.211$ \\
\hline Temperature & 14 & $5,535.72$ & 2.57 & 4.85 & $5,507.637$ \\
\hline Weight & 14 & $5,536.13$ & 2.97 & 5.25 & $5,508.038$ \\
\hline Temperature + Discharge & 15 & $5,533.48$ & 0.33 & 2.60 & $5,503.379$ \\
\hline Temperature $+\%$ Spill & 15 & $5,535.03$ & 1.88 & 4.15 & $5,504.929$ \\
\hline$\%$ Spill + Discharge & 15 & $5,536.24$ & 3.09 & 5.36 & $5,506.139$ \\
\hline$\%$ Spill + Discharge $+\%$ Spill $\times$ Discharge & 16 & $5,533.42$ & 0.27 & 2.54 & $5,501.306$ \\
\hline Weight + Temperature + Discharge & 16 & $5,533.66$ & 0.51 & 2.79 & $5,501.551$ \\
\hline Temperature + Discharge $+\%$ Spill & 16 & $5,535.12$ & 1.96 & 4.24 & $5,503.001$ \\
\hline Weight + Temperature $+\%$ Spill & 16 & $5,535.95$ & 2.80 & 5.07 & $5,503.837$ \\
\hline Weight $+\%$ Spill + Discharge & 16 & $5,536.12$ & 2.97 & 5.24 & $5,504.008$ \\
\hline Temperature + Discharge $+\%$ Spill $+\%$ Spill $\times$ Discharge & 17 & $5,533.15$ & 0.00 & 2.27 & $5,499.024$ \\
\hline Weight $+\%$ Spill + Discharge $+\%$ Spill $\times$ Discharge & 17 & $5,533.38$ & 0.23 & 2.50 & $5,499.254$ \\
\hline Weight + Temperature + Discharge $+\%$ Spill & 17 & $5,535.47$ & 2.32 & 4.59 & $5,501.342$ \\
\hline Weight + Temperature + Discharge $+\%$ Spill $+\%$ Spill $\times$ Discharge & 18 & $5,533.45$ & 0.30 & 2.57 & $5,497.309$ \\
\hline
\end{tabular}


Table 9 (continued). Model selection for fish that passed through the juvenile bypass system at McNary Dam relating survival $(\boldsymbol{o})$ as a function of environmental variables and group covariates for photoperiod using the fixed structure of the best model for $\boldsymbol{p}$ and $\lambda$ parameters for yearling Chinook salmon, 2004-09.

[The best-fit model is indicated in bold]

\begin{tabular}{|c|c|c|c|c|c|}
\hline Model & $\begin{array}{l}\text { Number of } \\
\text { parameters }\end{array}$ & $\mathrm{AlC} c$ & $\begin{array}{c}\Delta \mathrm{AIC} c \\
\text { (group) }\end{array}$ & $\begin{array}{c}\Delta \mathrm{AIC} c \\
\text { (overall) }\end{array}$ & Deviance \\
\hline Photoperiod & 14 & $5,532.34$ & 1.46 & 1.46 & 28.310 \\
\hline Photoperiod + Discharge & 15 & $5,532.08$ & 1.20 & 1.20 & $5,501.977$ \\
\hline Photoperiod + Temperature & 15 & $5,532.56$ & 1.68 & 1.68 & $5,502.463$ \\
\hline Photoperiod + Weight & 15 & $5,532.61$ & 1.74 & 1.74 & $5,502.514$ \\
\hline Photoperiod + Discharge + Temperature & 16 & $5,531.02$ & 0.14 & 0.14 & $5,498.903$ \\
\hline Photoperiod $+\%$ Spill & 15 & $5,533.01$ & 2.13 & 2.13 & $5,502.905$ \\
\hline Photoperiod + Weight + Discharge & 16 & $5,531.66$ & 0.78 & 0.78 & $5,499.542$ \\
\hline Photoperiod + Weight + Temperature & 16 & $5,533.32$ & 2.44 & 2.44 & $5,501.203$ \\
\hline Photoperiod + Weight $+\%$ Spill & 16 & $5,533.45$ & 2.57 & 2.57 & $5,501.335$ \\
\hline Photoperiod $+\%$ Spill + Discharge & 16 & $5,533.95$ & 3.07 & 3.07 & $5,501.835$ \\
\hline Photoperiod $+\%$ Spill + Discharge $+\%$ Spill $\times$ Discharge & 17 & $5,531.12$ & 0.24 & 0.24 & $5,496.992$ \\
\hline Photoperiod $+\%$ Spill + Discharge + Temperature & 17 & $5,533.03$ & 2.15 & 2.15 & $5,498.903$ \\
\hline Photoperiod + Weight $+\%$ Spill + Discharge & 17 & $5,533.66$ & 2.78 & 2.78 & $5,499.530$ \\
\hline Photoperiod + Weight $+\%$ Spill + Discharge $+\%$ Spill $\times$ Discharge & 18 & $5,530.88$ & 0.00 & 0.00 & $5,494.737$ \\
\hline Photoperiod $+\%$ Spill + Discharge + Temperature $+\%$ Spill $\times$ Discharge & 18 & $5,531.03$ & 0.15 & 0.15 & $5,494.885$ \\
\hline Photoperiod + Weight $+\%$ Spill + Discharge + Temperature & 18 & $5,533.26$ & 2.38 & 2.38 & $5,497.114$ \\
\hline Photoperiod + Weight $+\%$ Spill + Discharge + Temperature $+\%$ Spill $\times$ Discharge & 19 & $5,531.16$ & 0.28 & 0.28 & $5,493.005$ \\
\hline
\end{tabular}


Table 10. Model selection for fish that passed through the spillway at McNary Dam relating survival $(\varnothing)$ as a function of environmental variables using the fixed structure of the best model for $p$ and $\lambda$ parameters for yearling Chinook salmon, 2004-09.

\begin{tabular}{|c|c|c|c|c|c|}
\hline Model & $\begin{array}{l}\text { Number of } \\
\text { parameters }\end{array}$ & $\mathrm{AIC} c$ & $\begin{array}{l}\Delta \mathrm{AIC} c \\
\text { (group) }\end{array}$ & $\begin{array}{c}\Delta \mathrm{AIC} c \\
\text { (overall) }\end{array}$ & Deviance \\
\hline Intercept only & 13 & $10,033.42$ & 5.86 & 12.06 & 110.520 \\
\hline Weight & 14 & $10,029.52$ & 1.96 & 8.16 & $10,001.472$ \\
\hline Discharge & 14 & $10,032.62$ & 5.07 & 11.27 & $10,004.579$ \\
\hline Temperature & 14 & $10,033.32$ & 5.77 & 11.97 & $10,005.274$ \\
\hline$\%$ Spill & 14 & $10,035.15$ & 7.60 & 13.80 & $10,007.103$ \\
\hline Temperature + Discharge & 15 & $10,034.26$ & 6.71 & 12.91 & $10,004.207$ \\
\hline$\%$ Spill + Discharge & 15 & $10,034.62$ & 7.07 & 13.27 & $10,004.566$ \\
\hline Temperature $+\%$ Spill & 15 & $10,035.33$ & 7.77 & 13.97 & $10,005.274$ \\
\hline Weight + Discharge & 15 & $10,026.21$ & 1.34 & 4.85 & $9,996.155$ \\
\hline Weight + Temperature + Discharge & 16 & $10,027.55$ & 0.00 & 6.20 & $9,995.494$ \\
\hline Weight $+\%$ Spill + Discharge & 16 & $10,028.04$ & 0.49 & 6.69 & $9,995.981$ \\
\hline Weight + Temperature $+\%$ Spill & 16 & $10,029.78$ & 2.22 & 8.42 & $9,997.717$ \\
\hline$\%$ Spill + Discharge $+\%$ Spill $\times$ Discharge & 16 & $10,035.49$ & 7.94 & 14.14 & $10,003.431$ \\
\hline Temperature + Discharge $+\%$ Spill & 16 & $10,036.26$ & 8.71 & 14.91 & $10,004.205$ \\
\hline Weight $+\%$ Spill + Discharge $+\%$ Spill $\times$ Discharge & 17 & $10,029.17$ & 1.62 & 7.82 & $9,995.105$ \\
\hline Weight + Temperature + Discharge $+\%$ Spill & 17 & $10,029.50$ & 1.95 & 8.15 & $9,995.434$ \\
\hline Temperature + Discharge $+\%$ Spill $+\%$ Spill $\times$ Discharge & 17 & $10,037.21$ & 9.66 & 15.86 & $10,003.143$ \\
\hline Weight + Temperature + Discharge $+\%$ Spill $+\%$ Spill $\times$ Discharge & 18 & $10,030.72$ & 3.16 & 9.36 & $9,994.642$ \\
\hline
\end{tabular}


Table 10 (continued). Model selection for fish that passed through the spillway at McNary Dam relating survival $(\boldsymbol{o})$ as a function of environmental variables and group covariates for photoperiod using the fixed structure of the best model for $\boldsymbol{p}$ and $\lambda$ parameters for yearling Chinook salmon, 2004-09.

\begin{tabular}{lrrrrr}
\hline \multicolumn{1}{c}{ Model } & $\begin{array}{c}\text { Number of } \\
\text { parameters }\end{array}$ & AlC $\boldsymbol{c}$ & \multicolumn{1}{c}{$\begin{array}{c}\Delta \text { AIC } \boldsymbol{c} \\
\text { (group) }\end{array}$} & \multicolumn{1}{c}{$\begin{array}{c}\Delta \text { AIC } \boldsymbol{c} \\
\text { (overall) }\end{array}$} & \multicolumn{1}{c}{ Deviance } \\
\hline Photoperiod & 14 & $10,034.73$ & 6.64 & 13.37 & 109.826 \\
Photoperiod + Discharge & 15 & $10,034.11$ & 6.02 & 12.75 & $10,004.055$ \\
Photoperiod + Weight & 15 & $10,031.20$ & 3.11 & 9.85 & $10,001.152$ \\
Photoperiod + \%Spill & 15 & $10,036.13$ & 8.04 & 14.78 & $10,006.076$ \\
Photoperiod + Weight + Discharge & 16 & $10,028.09$ & 0.00 & 6.74 & $9,996.031$ \\
Photoperiod + Temperature & 15 & $10,034.67$ & 6.58 & 13.32 & $10,004.622$ \\
Photoperiod + \%Spill + Discharge & 16 & $10,035.98$ & 7.89 & 14.63 & $10,003.924$ \\
Photoperiod + Weight + Temperature & 16 & $10,029.65$ & 1.56 & 8.29 & $9,997.586$ \\
Photoperiod + Weight + \%Spill & 16 & $10,032.00$ & 3.91 & 10.65 & $9,999.945$ \\
Photoperiod + Weight + \%Spill + Discharge & 17 & $10,029.79$ & 1.70 & 8.43 & $9,995.719$ \\
Photoperiod + \%Spill + Discharge + \%Spill $\times$ Discharge & 17 & $10,036.95$ & 8.86 & 15.60 & $10,002.885$ \\
Photoperiod + \%Spill + Discharge + Temperature & 17 & $10,037.69$ & 9.60 & 16.34 & $10,003.622$ \\
Photoperiod + Weight + \%Spill + Discharge + \%Spill $\times$ Discharge & 18 & $10,030.97$ & 2.88 & 9.61 & $9,994.892$ \\
Photoperiod + \%Spill + Discharge + Temperature + \%Spill $\times$ Discharge & 18 & $10,038.72$ & 10.63 & 17.36 & $10,002.641$ \\
Photoperiod + Weight + \%Spill + Discharge + Temperature & 18 & $10,031.30$ & 3.21 & 9.95 & $9,995.225$ \\
Photoperiod + Weight + \%Spill + Discharge + Temperature + \%Spill $\times$ Discharge & 19 & $10,032.55$ & 4.46 & 11.20 & $9,994.470$ \\
\hline
\end{tabular}


Table 10 (continued). Model selection for fish that passed through the spillway at McNary Dam relating survival $(\boldsymbol{\theta})$ as a function of environmental variables and group covariates for spillway passage location (i.e. north, south) using the fixed structure of the best model for $\boldsymbol{p}$ and $\lambda$ parameters for yearling Chinook salmon, 2004-09.

[The best-fit model is indicated in bold]

\begin{tabular}{|c|c|c|c|c|c|}
\hline Model & $\begin{array}{l}\text { Number of } \\
\text { parameters }\end{array}$ & $\mathrm{AIC} c$ & $\begin{array}{l}\Delta \mathrm{AIC} c \\
\text { (group) }\end{array}$ & $\begin{array}{c}\Delta \mathrm{AIC} c \\
\text { (overall) }\end{array}$ & Deviance \\
\hline SpillPassLoc & 15 & $10,027.58$ & 6.23 & 6.23 & 100.673 \\
\hline SpillPassLoc + Discharge & 16 & $10,027.49$ & 6.13 & 6.13 & $9,995.426$ \\
\hline SpillPassLoc $+\%$ Spill & 16 & $10,029.12$ & 7.77 & 7.77 & $9,997.059$ \\
\hline SpillPassLoc + Temperature & 16 & $10,027.95$ & 6.60 & 6.60 & $9,995.893$ \\
\hline SpillPassLoc + Weight & 16 & $10,023.53$ & 2.17 & 2.17 & $9,991.466$ \\
\hline SpillPassLoc + Weight + Discharge & 17 & $10,021.35$ & 0.00 & 0.00 & $9,987.287$ \\
\hline SpillPassLoc + Weight $+\%$ Spill & 17 & $10,024.54$ & 3.18 & 3.18 & $9,990.470$ \\
\hline SpillPassLoc + Temperature + Discharge & 17 & $10,029.17$ & 7.82 & 7.82 & $9,995.105$ \\
\hline SpillPassLoc $+\%$ Spill + Discharge & 17 & $10,029.38$ & 8.03 & 8.03 & $9,995.312$ \\
\hline SpillPassLoc + Temperature $+\%$ Spill & 17 & $10,029.89$ & 8.53 & 8.53 & $9,995.819$ \\
\hline SpillPassLoc + Weight + Temperature + Discharge & 18 & $10,022.83$ & 1.48 & 1.48 & $9,986.755$ \\
\hline SpillPassLoc + Weight $+\%$ Spill + Discharge & 18 & $10,023.07$ & 1.71 & 1.71 & $9,986.993$ \\
\hline SpillPassLoc $+\%$ Spill + Discharge $+\%$ Spill $\times$ Discharge & 18 & $10,030.43$ & 9.07 & 9.07 & $9,994.353$ \\
\hline SpillPassLoc + Temperature + Discharge $+\%$ Spill & 18 & $10,031.14$ & 9.78 & 9.78 & $9,995.061$ \\
\hline SpillPassLoc + Weight $+\%$ Spill + Discharge $+\%$ Spill $\times$ Discharge & 19 & $10,024.39$ & 3.04 & 3.04 & $9,986.307$ \\
\hline SpillPassLoc + Weight + Temperature + Discharge $+\%$ Spill & 19 & $10,024.67$ & 3.32 & 3.32 & $9,986.587$ \\
\hline SpillPassLoc + Temperature + Discharge $+\%$ Spill $+\%$ Spill $\times$ Discharge & 19 & $10,032.24$ & 10.89 & 10.89 & $9,994.156$ \\
\hline SpillPassLoc + Weight + Temperature + Discharge $+\%$ Spill $+\%$ Spill $\times$ Discharge & 20 & $10,026.06$ & 4.70 & 4.70 & $9,985.965$ \\
\hline
\end{tabular}


Table 10 (continued). Model selection for fish that passed through the spillway at McNary Dam relating survival $(\boldsymbol{\theta})$ as a function of environmental variables and group covariates for spillway passage location (i.e. north, south) and photoperiod using the fixed structure of the best model for $\boldsymbol{p}$ and $\lambda$ parameters for yearling Chinook salmon, 2004-09.

\begin{tabular}{|c|c|c|c|c|c|}
\hline Model & $\begin{array}{l}\text { Number of } \\
\text { parameters }\end{array}$ & $\mathrm{AIC} c$ & $\begin{array}{l}\Delta \mathrm{AIC} c \\
\text { group }\end{array}$ & $\begin{array}{l}\Delta \mathrm{AIC} c \\
\text { overall }\end{array}$ & Deviance \\
\hline SpillPassLoc + Photoperiod & 16 & $10,029.32$ & 5.97 & 7.97 & 100.41 \\
\hline SpillPassLoc + Photoperiod + Discharge & 17 & $10,029.31$ & 5.96 & 7.96 & $9,995.25$ \\
\hline SpillPassLoc + Photoperiod + Temperature & 17 & $10,029.71$ & 6.36 & 8.36 & $9,995.64$ \\
\hline SpillPassLoc + Photoperiod + Weight & 17 & $10,025.47$ & 2.11 & 4.11 & $9,991.40$ \\
\hline SpillPassLoc + Photoperiod $+\%$ Spill & 17 & $10,030.60$ & 7.25 & 9.25 & $9,996.54$ \\
\hline SpillPassLoc + Photoperiod + Weight + Discharge & 18 & $10,023.35$ & 0.00 & 2.00 & $9,987.28$ \\
\hline SpillPassLoc + Photoperiod + Weight + Temperature & 18 & $10,024.61$ & 1.26 & 3.26 & $9,988.54$ \\
\hline SpillPassLoc + Photoperiod + Weight $+\%$ Spill & 18 & $10,026.25$ & 2.90 & 4.90 & $9,990.18$ \\
\hline SpillPassLoc + Photoperiod $+\%$ Spill + Discharge & 18 & $10,031.08$ & 7.73 & 9.73 & $9,995.01$ \\
\hline SpillPassLoc + Photoperiod + Weight $+\%$ Spill + Discharge & 19 & $10,025.01$ & 1.65 & 3.65 & $9,986.92$ \\
\hline SpillPassLoc + Photoperiod $+\%$ Spill + Discharge $+\%$ Spill $\times$ Discharge & 19 & $10,032.18$ & 8.83 & 10.83 & $9,994.10$ \\
\hline SpillPassLoc + Photoperiod $+\%$ Spill + Discharge + Temperature & 19 & $10,032.87$ & 9.51 & 11.51 & $9,994.78$ \\
\hline SpillPassLoc + Photoperiod + Weight $+\%$ Spill + Discharge $+\%$ Spill $\times$ Discharge & 20 & $10,026.35$ & 2.99 & 4.99 & $9,986.26$ \\
\hline SpillPassLoc + Photoperiod + Weight + Photoperiod $+\%$ Spill + Discharge + Temperature & 20 & $10,026.63$ & 3.27 & 5.27 & $9,986.54$ \\
\hline SpillPassLoc + Photoperiod $+\%$ Spill + Discharge + Temperature $+\%$ Spill $\times$ Discharge & 20 & $10,034.02$ & 10.66 & 12.66 & $9,993.92$ \\
\hline SpillPassLoc + Photoperiod + Weight + Photoperiod $+\%$ Spill + Discharge + Temperature $+\%$ Spill $\times$ Discharge & 21 & $10,028.03$ & 4.67 & 6.67 & $9,985.93$ \\
\hline
\end{tabular}



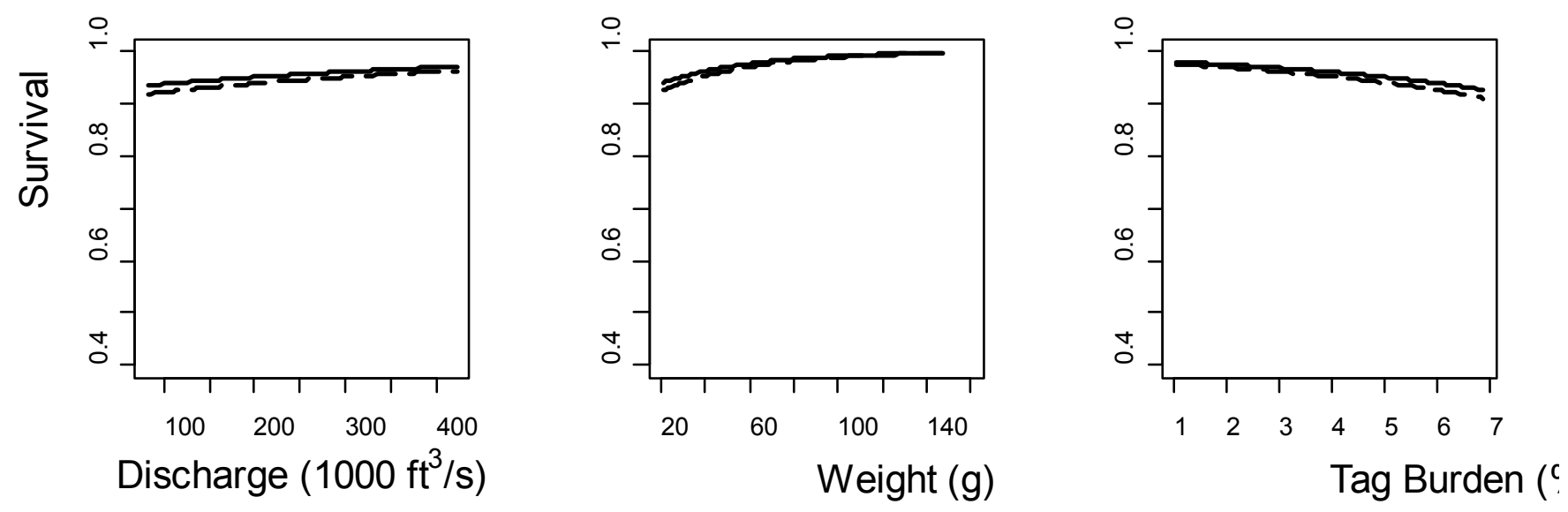

Figure 11. Survival of yearling Chinook salmon that passed through the north (solid line) and south (dashed line) portion of the spillway at McNary Dam in relation to total discharge, fish weight, and tag burden (tag burden=tag weightffish weight $\times 100,2004-09$. Other covariates in the model were held constant at their mean value to examine the relationship to the variable of interest. 
Table 11. Model selection for fish released in the tailrace of McNary Dam relating survival $(\varnothing)$ as a function of environmental variables using the fixed structure of the best model for $p$ and $\lambda$ parameters for yearling Chinook salmon, 2004-09.

[The best-fit model is indicated in bold]

\begin{tabular}{|c|c|c|c|c|c|}
\hline Model & $\begin{array}{l}\text { Number of } \\
\text { parameters }\end{array}$ & $\mathrm{AIC} c$ & $\begin{array}{l}\Delta \mathrm{AIC} c \\
\text { (group) }\end{array}$ & $\begin{array}{c}\Delta \mathrm{AIC} c \\
\text { (overall) }\end{array}$ & Deviance \\
\hline Intercept only & 13 & $11,391.16$ & 0.00 & 0.00 & 75.862 \\
\hline$\%$ Spill & 14 & $11,392.54$ & 1.38 & 1.38 & $11,364.505$ \\
\hline Temperature & 14 & $11,392.56$ & 1.41 & 1.41 & $11,364.528$ \\
\hline Weight & 14 & $11,392.61$ & 1.45 & 1.45 & $11,364.575$ \\
\hline Discharge & 14 & $11,392.84$ & 1.68 & 1.68 & $11,364.801$ \\
\hline Temperature + Discharge & 15 & $11,393.17$ & 2.01 & 2.01 & $11,363.123$ \\
\hline Temperature $+\%$ Spill & 15 & $11,393.71$ & 2.55 & 2.55 & $11,363.669$ \\
\hline$\%$ Spill + Discharge & 15 & $11,394.52$ & 3.36 & 3.36 & $11,364.477$ \\
\hline Weight + Temperature + Discharge & 16 & $11,394.61$ & 3.45 & 3.45 & $11,362.560$ \\
\hline Temperature + Discharge $+\%$ Spill & 16 & $11,395.08$ & 3.92 & 3.92 & $11,363.031$ \\
\hline Weight + Temperature $+\%$ Spill & 16 & $11,395.27$ & 4.11 & 4.11 & $11,363.221$ \\
\hline Weight $+\%$ Spill + Discharge & 16 & $11,395.72$ & 4.56 & 4.56 & $11,363.671$ \\
\hline$\%$ Spill + Discharge $+\%$ Spill $\times$ Discharge & 16 & $11,396.50$ & 5.34 & 5.34 & $11,364.456$ \\
\hline Weight + Temperature + Discharge $+\%$ Spill & 17 & $11,396.51$ & 5.36 & 5.36 & $11,362.461$ \\
\hline Temperature + Discharge $+\%$ Spill $+\%$ Spill $\times$ Discharge & 17 & $11,397.08$ & 5.92 & 5.92 & $11,363.029$ \\
\hline Weight $+\%$ Spill + Discharge $+\%$ Spill $\times$ Discharge & 17 & $11,397.70$ & 6.54 & 6.54 & $11,363.643$ \\
\hline Weight + Temperature + Discharge $+\%$ Spill $+\%$ Spill $\times$ Discharge & 18 & $11,398.52$ & 7.36 & 7.36 & $11,362.461$ \\
\hline
\end{tabular}


Table 11 (continued). Model selection for fish released in the tailrace of McNary Dam relating survival ( $\boldsymbol{\theta})$ as a function of environmental variables and group covariates for photoperiod using the fixed structure of the best model for $\boldsymbol{p}$ and $\lambda$ parameters for yearling Chinook salmon, 2004-09.

\begin{tabular}{lccccc}
\hline \multicolumn{1}{c}{ Model } & $\begin{array}{c}\text { Number of } \\
\text { parameters }\end{array}$ & AlC $\boldsymbol{c}$ & $\begin{array}{c}\Delta \text { AIC } \boldsymbol{c} \\
\text { (group) }\end{array}$ & $\begin{array}{c}\Delta \text { AIC } \boldsymbol{c} \\
\text { (overall) }\end{array}$ & \multicolumn{1}{c}{ Deviance } \\
\hline Photoperiod & 14 & $11,392.04$ & 0.00 & 0.88 & 74.739 \\
Photoperiod + Discharge & 15 & $11,393.43$ & 1.39 & 2.27 & $11,363.389$ \\
Photoperiod + \%Spill & 15 & $11,393.60$ & 1.56 & 2.44 & $11,363.556$ \\
Photoperiod + Weight & 15 & $11,393.64$ & 1.60 & 2.49 & $11,363.602$ \\
Temperature + Photoperiod & 15 & $11,393.72$ & 1.67 & 2.56 & $11,363.673$ \\
Weight + Photoperiod + Discharge & 16 & $11,394.73$ & 2.68 & 3.57 & $11,362.679$ \\
Weight + Photoperiod + \%Spill & 16 & $11,395.07$ & 3.03 & 3.91 & $11,363.024$ \\
Photoperiod + \%Spill + Discharge & 16 & $11,395.38$ & 3.34 & 4.23 & $11,363.337$ \\
Weight + Temperature + Photoperiod & 16 & $11,395.44$ & 3.40 & 4.28 & $11,363.396$ \\
Photoperiod + \%Spill + Discharge + Temperature & 17 & $11,396.11$ & 4.07 & 4.96 & $11,362.061$ \\
Weight + Photoperiod + \%Spill + Discharge & 17 & $11,396.69$ & 4.65 & 5.53 & $11,362.635$ \\
Photoperiod + \%Spill + Discharge + \%Spill $\times$ Discharge & 17 & $11,397.38$ & 5.34 & 6.22 & $11,363.324$ \\
Weight + Photoperiod + \%Spill + Discharge + Temperature & 18 & $11,397.62$ & 5.58 & 6.46 & $11,361.557$ \\
Photoperiod + \%Spill + Discharge + Temperature + \%Spill $\times$ Discharge & 18 & $11,398.11$ & 6.07 & 6.96 & $11,362.055$ \\
Weight + Photoperiod + \%Spill + Discharge + \%Spill $\times$ Discharge & 18 & $11,398.68$ & 6.64 & 7.52 & $11,362.618$ \\
Weight + Photoperiod + \%Spill + Discharge + Temperature +\%Spill $\times$ Discharge & 19 & $11,399.62$ & 7.58 & 8.46 & $11,361.556$ \\
\hline
\end{tabular}




\section{Temporal Survival Estimates}

Survival estimates at the weekly (table A1) and annual (table 12) temporal scale were generated to provide temporal information for survival through routes and to compare estimates predicted by covariates. Weekly survival was relatively constant for all years, especially for fish that migrated through the forebay, passed through the spillway, or were released in the tailrace of McNary Dam (figs. 12 and 13). Survival in the forebay decreased substantially during late spring of 2005 (fig. 12). Weekly survival for fish that passed through the juvenile bypass system and turbines was much more variable (fig. 13). Weekly survival for yearling Chinook salmon that went through turbines ranged from 0.622 to 1.00. Furthermore, weekly survival for fish that passed the juvenile bypass system ranged from 0.794 to 1.00. In contrast, survival for fish released in the tailrace of McNary Dam ranged from 0.918 to 1.00 and those that passed through the spillway ranged from 0.851 to 1.00 . Covariates predicted weekly survival estimates well for fish that migrated through the forebay or passed through the spillway, but not so well for fish that passed through the turbines (figs. 14 and 15). Survival of fish that passed through the juvenile bypass or that were released in the tailrace were not significantly related to individual covariates, therefore, model predictions were not presented. At the annual temporal scale, covariates predicted survival better than at the weekly temporal scale (figs. 14 and 15).

Table 12. Annual survival estimates during the spring migration period for yearling Chinook salmon passing through and released in the tailrace of McNary Dam, 2004-09.

[Standard error is in parentheses]

\begin{tabular}{lcccccc}
\hline & $\mathbf{2 0 0 4}$ & $\mathbf{2 0 0 5}$ & $\mathbf{2 0 0 6}$ & $\mathbf{2 0 0 7}$ & $\mathbf{2 0 0 8}$ & $\mathbf{2 0 0 9}$ \\
\hline Forebay & NA & $0.965(0.004)$ & $0.991(0.003)$ & $0.992(0.003)$ & $0.999(0.001)$ & $0.995(0.002)$ \\
Turbine & $0.827(0.038)$ & $0.869(0.021)$ & $0.832(0.026)$ & $0.812(0.033)$ & $0.928(0.024)$ & $0.871(0.025)$ \\
Bypass & $0.858(0.030)$ & $0.931(0.010)$ & $0.943(0.013)$ & $0.961(0.031)$ & $0.957(0.014)$ & $0.954(0.010)$ \\
Spillway & $0.958(0.019)$ & $0.953(0.009)$ & $0.952(0.007)$ & $0.929(0.015)$ & $0.950(0.008)$ & $0.957(0.008)$ \\
Tailrace/Control & $0.975(0.017)$ & $0.975(0.005)$ & $0.977(0.006)$ & $0.997(0.015)$ & $0.988(0.005)$ & $0.964(0.006)$ \\
\hline
\end{tabular}




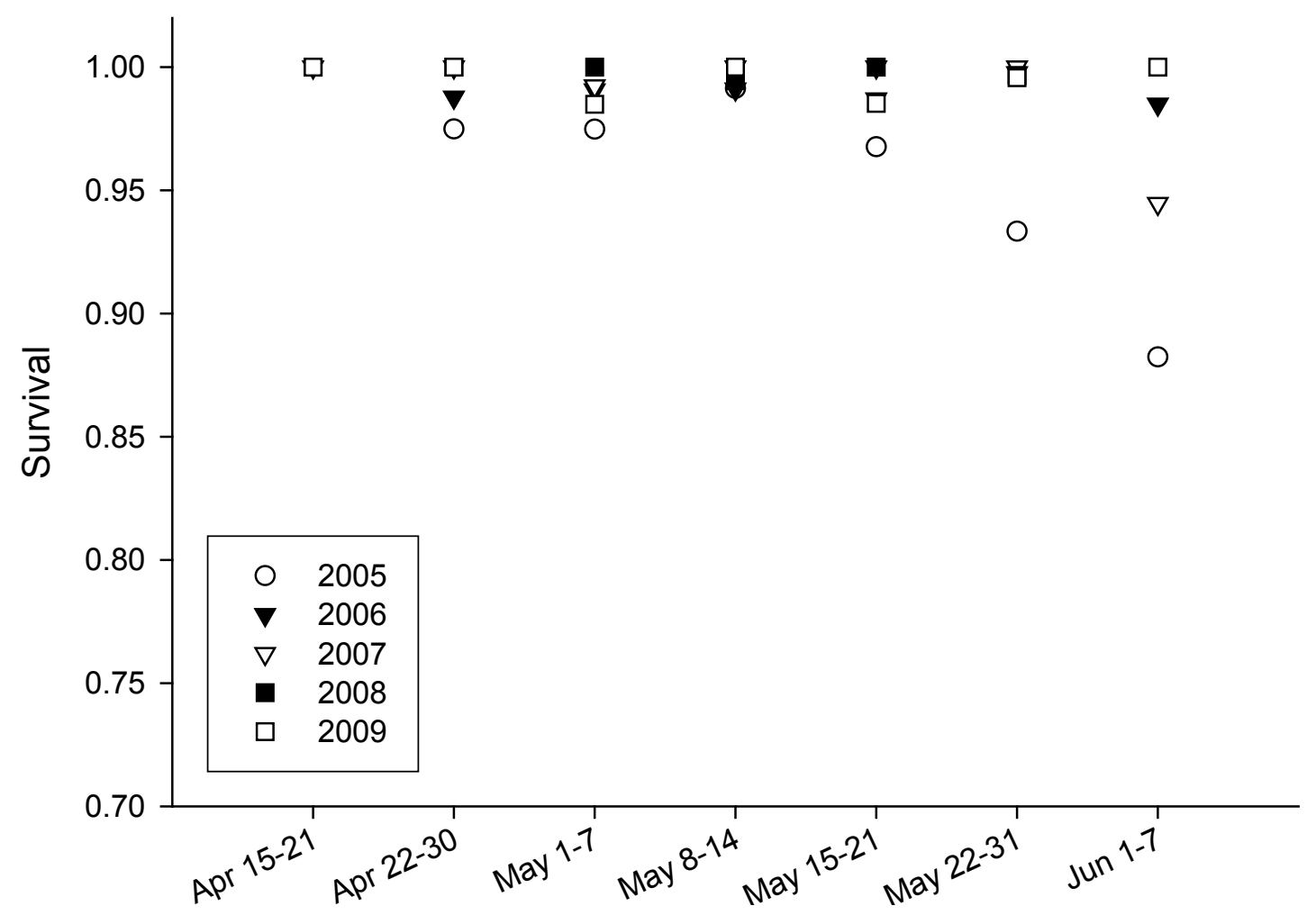

Figure 12. Weekly survival of yearling Chinook salmon in the forebay of McNary Dam, 2005-09. 
Turbine

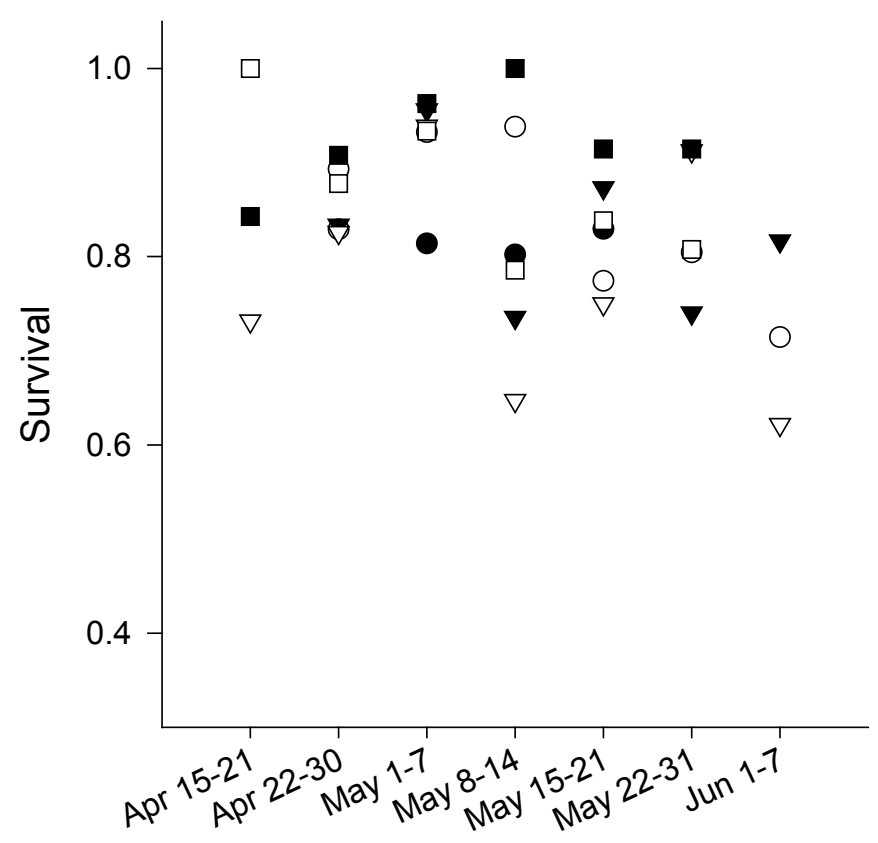

Spillway

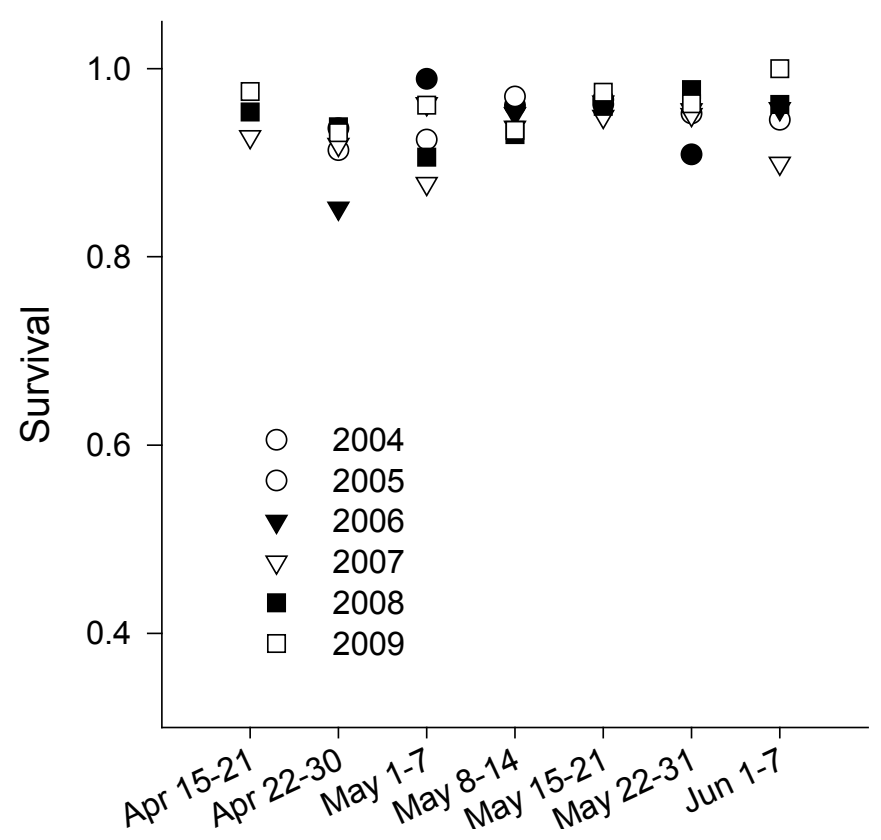

Bypass

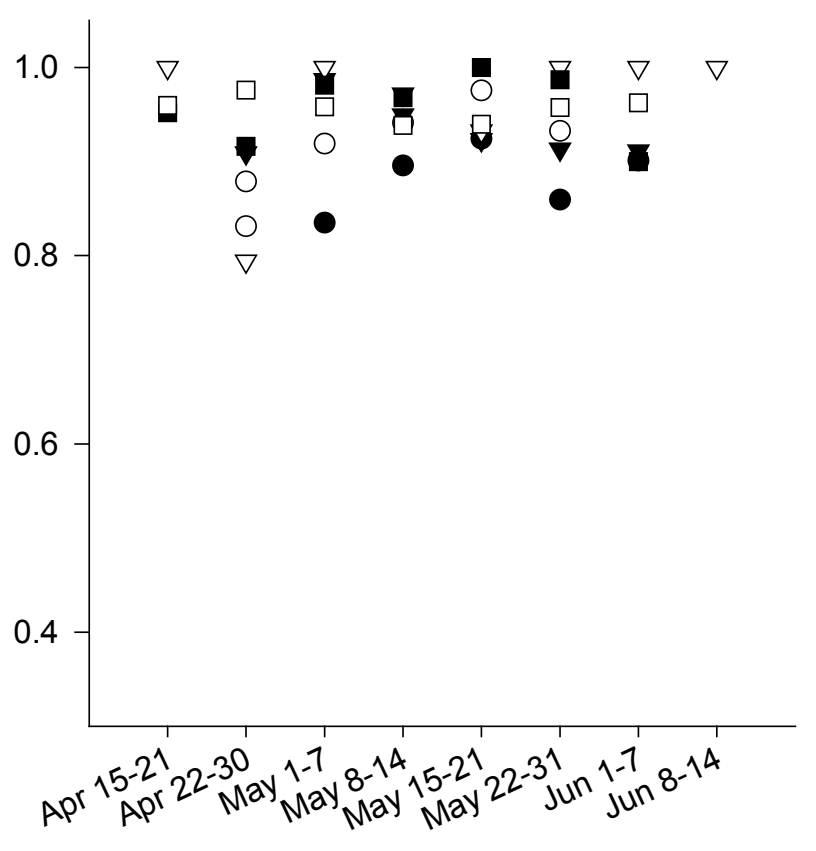

Tailrace/Control

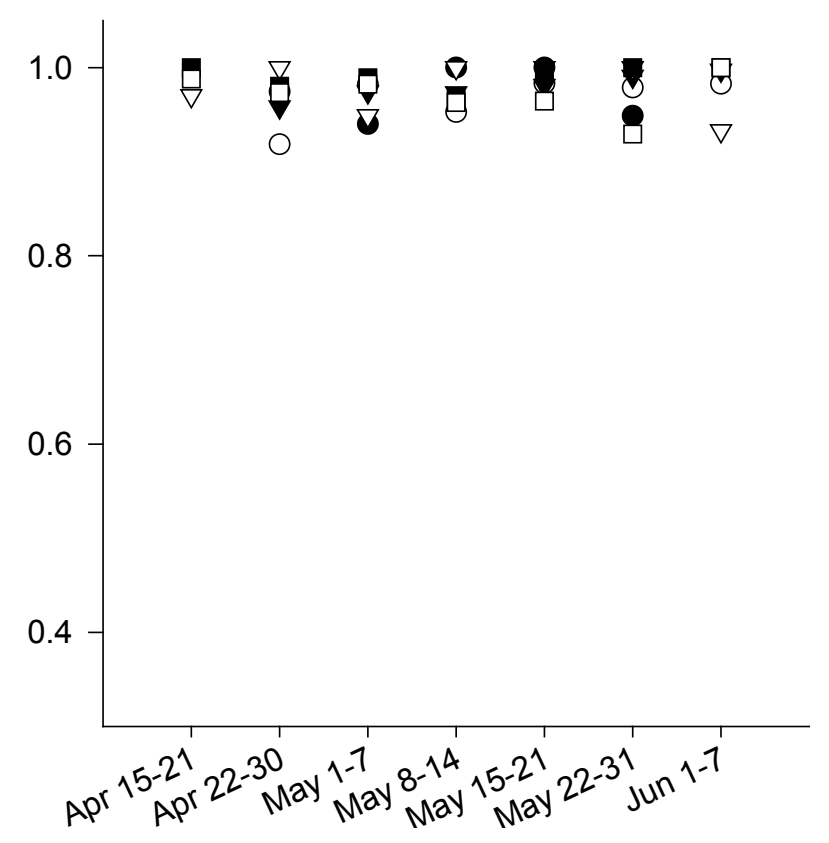

Figure 13. Weekly survival of yearling Chinook salmon for fish that passed through the juvenile bypass system, spillway, turbines, or that were released in the tailrace of McNary Dam, 2004-09. 

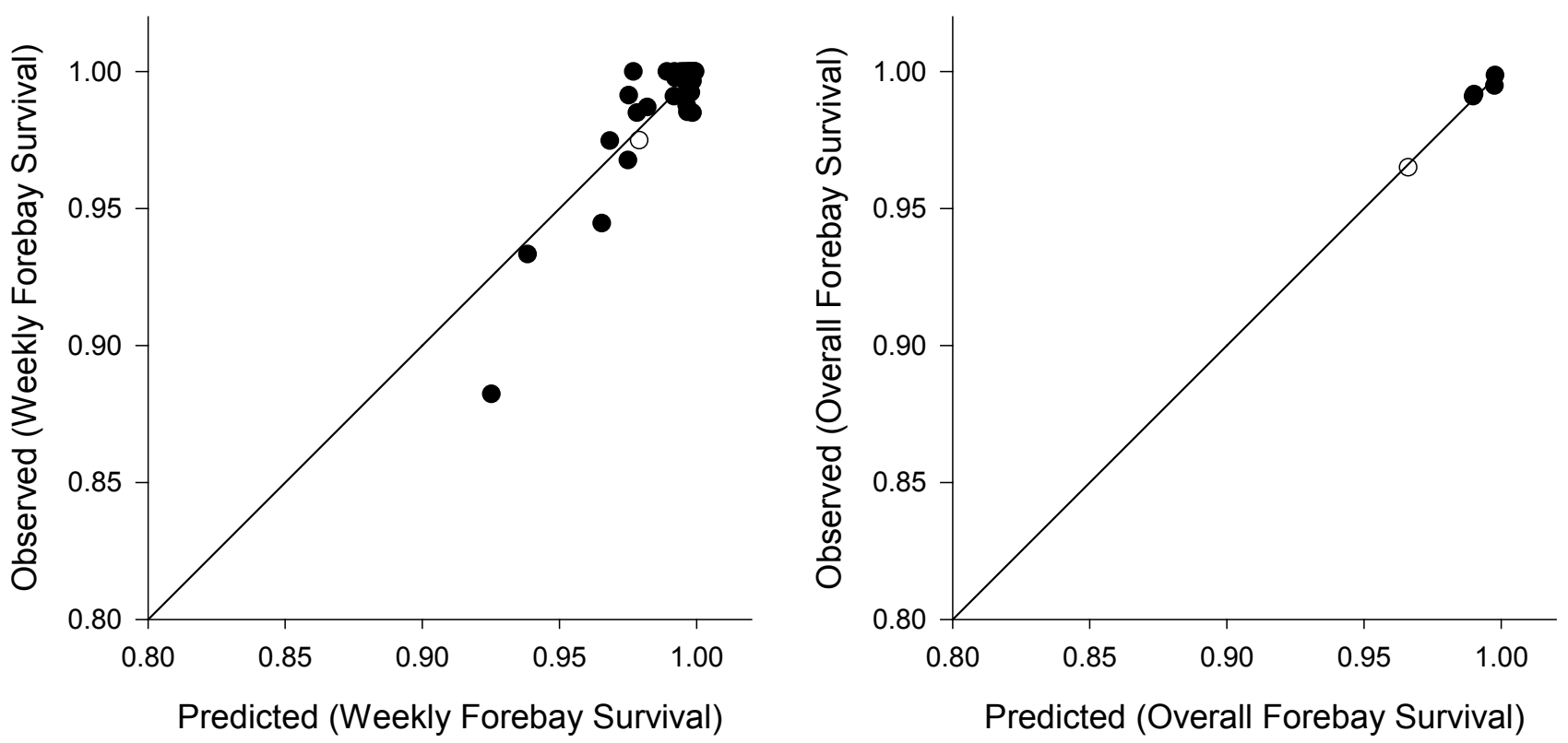

Figure 14. Observed versus predicted weekly (left) and annual survival (right) of yearling Chinook salmon during the spring migration period in the forebay of McNary Dam, 2005-09. Solid line indicates graph coordinates where observed and predicted survival are equivalent. 
Turbine
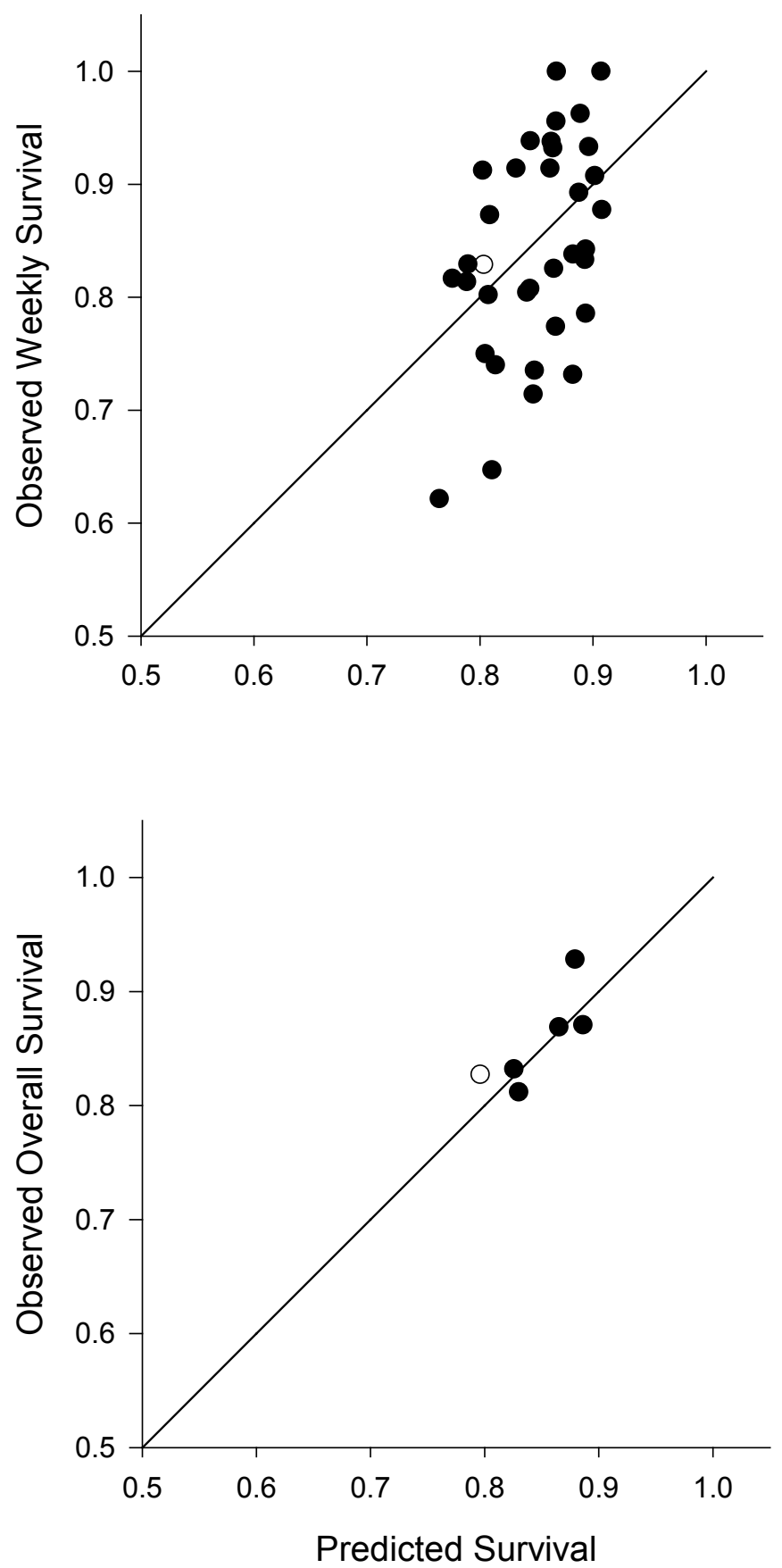

Spillway
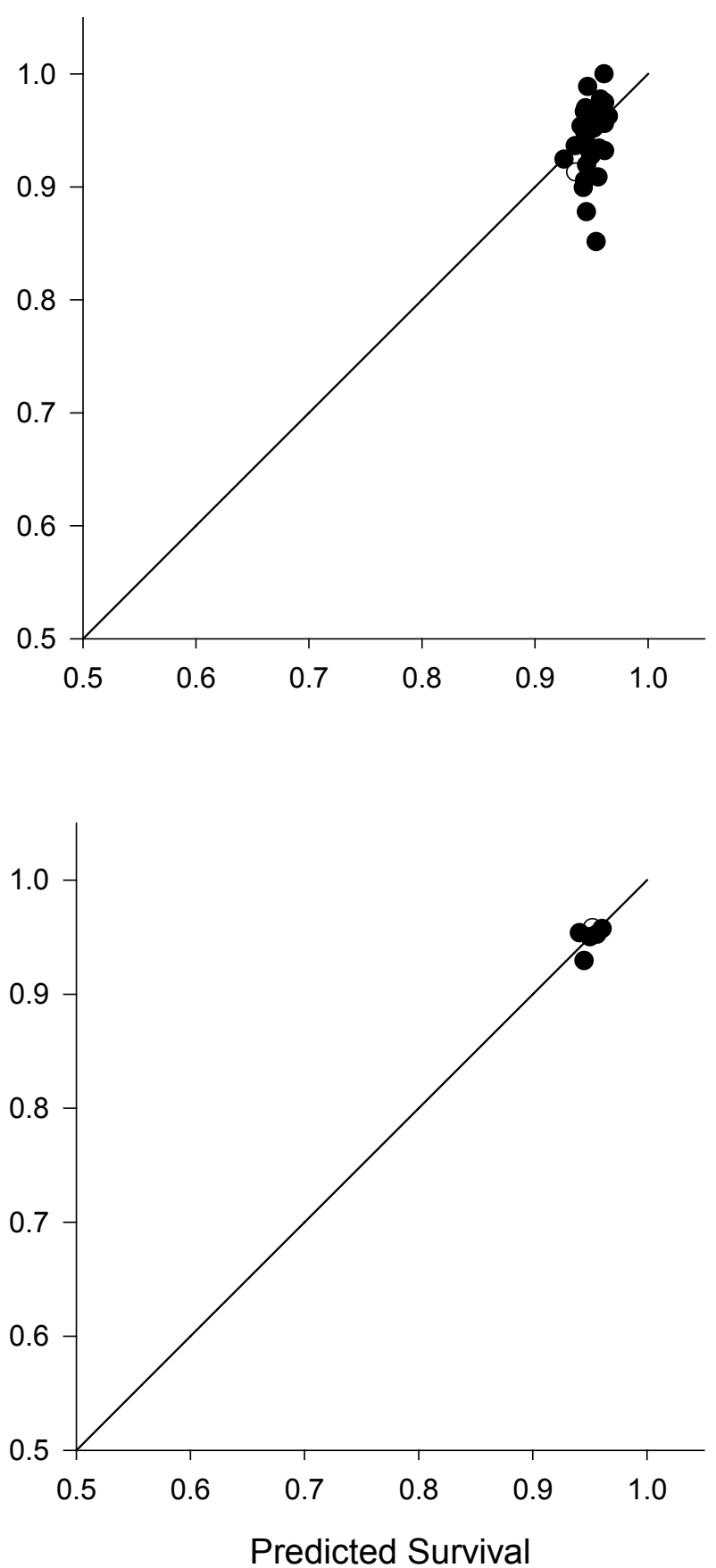

Figure 15. Observed versus predicted weekly (top) and annual survival (bottom) of yearling Chinook salmon during the spring migration period for fish that passed through the turbines (left) and spillway (right) of McNary Dam, 2004-09. Solid line indicates graph coordinates where observed and predicted survival are equivalent. 


\section{Juvenile Steelhead}

\section{Passage Analysis}

The best model explaining passage probabilities for juvenile steelhead at McNary Dam included covariates for percent spill, total discharge, and the interaction between percent spill and total discharge for all routes (table 13). As percent spill increased, more fish passed the dam through the spillway, and less passed through both turbines and the juvenile bypass system (fig. 16). As total discharge increased, more fish passed through the spillway and less passed through turbines. With increased discharge, more fish also passed through the bypass system when percent spill was low and more passed through turbines during low discharge and low percent spill conditions (fig. 17). Furthermore, passage through the juvenile bypass system was lower during the day (table 14). Fish weight was significant for fish passing through the spillway and showed that larger fish had a higher probability of passing the dam through the spillway (tables 13 and 14, fig. 18). Temperature also was significant in explaining passage probabilities through the spillway (table 13 and fig. 19). A factor for whether a TSW was installed explained a significant amount of variation for passage and was positively $\left(\beta_{\mathrm{TSW}}=1.016\right)$ related to passage through the spillway only when a year factor was not included.

Table 13. Passage models for juvenile steelhead that passed through McNary Dam, 2004-09, comparing the full model with models that have one variable removed.

[NLL, negative log likelihood; Df, degrees of freedom; $\chi^{2}$, chi-square statistic for likelihood ratio test]

\begin{tabular}{|c|c|c|c|c|c|c|}
\hline Model & $\begin{array}{c}\text { Number of } \\
\text { parameters }\end{array}$ & $\mathrm{AIC} c$ & NLL & $\chi^{2}$ & Df & $p$ \\
\hline \multicolumn{7}{|c|}{ Spillway } \\
\hline Full (all variables) & 14 & $10,536.42$ & $5,254.21$ & NA & NA & NA \\
\hline - Photoperiod & 13 & $10,542.26$ & $5,258.13$ & 7.84 & 1 & 0.0051 \\
\hline - \%Spill & 13 & $10,543.92$ & $5,258.96$ & 9.5 & 1 & 0.0021 \\
\hline - Discharge & 13 & $10,571.46$ & $5,272.73$ & 37.04 & 1 & 0 \\
\hline - Weight & 13 & $10,545.1$ & $5,259.55$ & 10.67 & 1 & 0.0011 \\
\hline - Temperature & 13 & $10,539.67$ & $5,256.83$ & 5.25 & 1 & 0.0219 \\
\hline - \%Spill $\times$ Discharge & 13 & $10,548.14$ & $5,261.07$ & 13.72 & 1 & $2.00 \times 10^{-4}$ \\
\hline \multicolumn{7}{|c|}{ Bypass } \\
\hline - Photoperiod & 13 & $10,576.22$ & $5,275.11$ & 41.8 & 1 & 0 \\
\hline - \%Spill & 13 & $10,567.51$ & $5,270.76$ & 33.09 & 1 & 0 \\
\hline - Discharge & 13 & $10,569.39$ & $5,271.7$ & 34.97 & 1 & 0 \\
\hline - Weight & 13 & $10,536.8$ & $5,255.4$ & 2.37 & 1 & 0.1237 \\
\hline - Temperature & 13 & $10,534.8$ & $5,254.4$ & 0.37 & 1 & 0.543 \\
\hline - \%Spill $\times$ Discharge & 13 & $10,562.47$ & $5,268.23$ & 28.05 & 1 & 0 \\
\hline
\end{tabular}




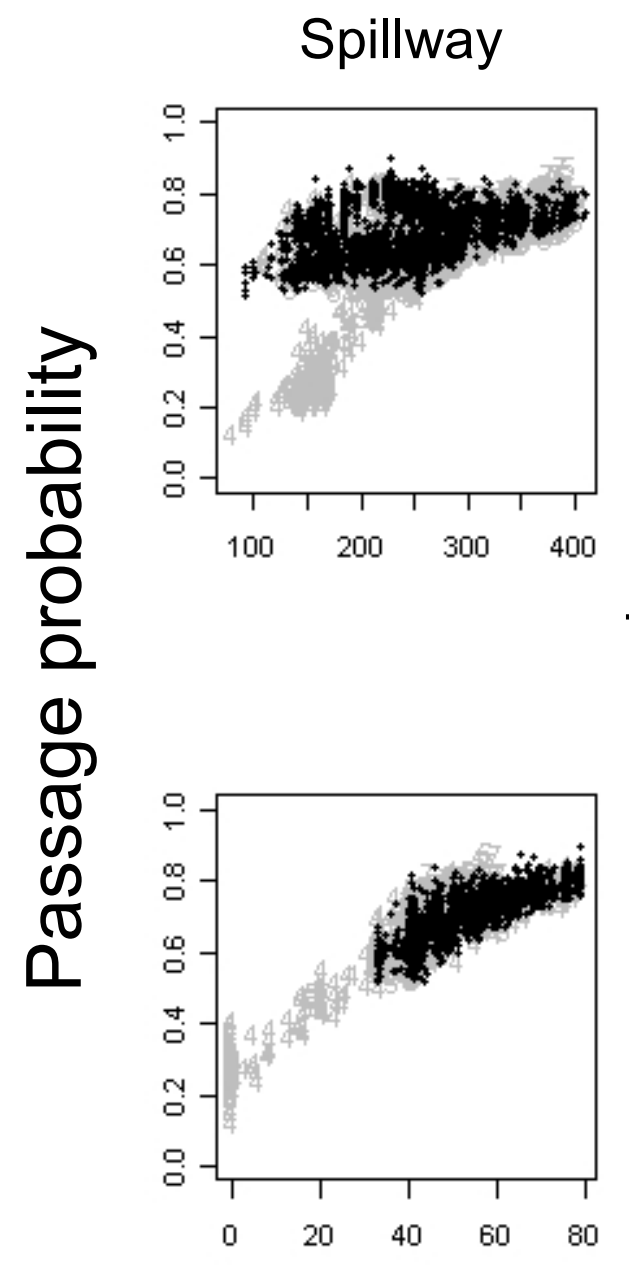

Turbines

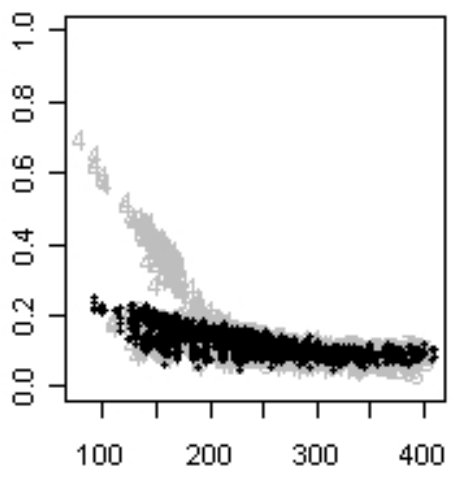

Total Discharge $\left(1000 \mathrm{ft}^{3} / \mathrm{s}\right)$
Bypass

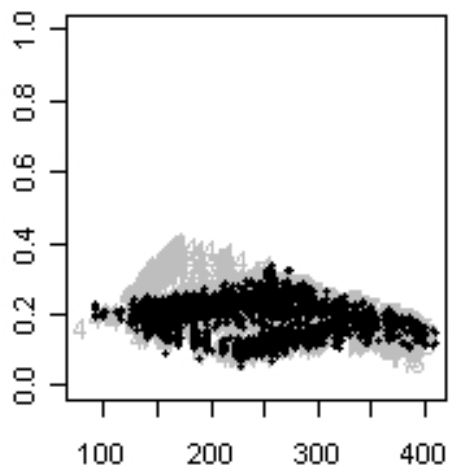

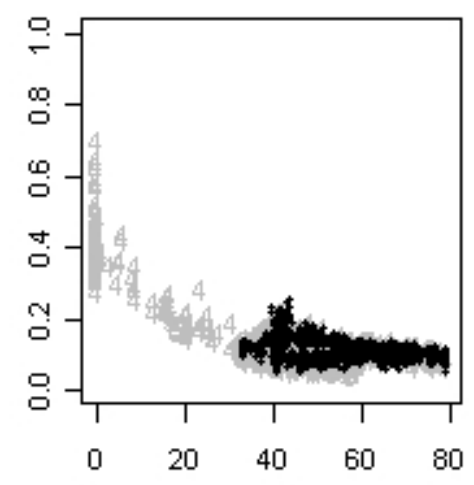

Percent Spill

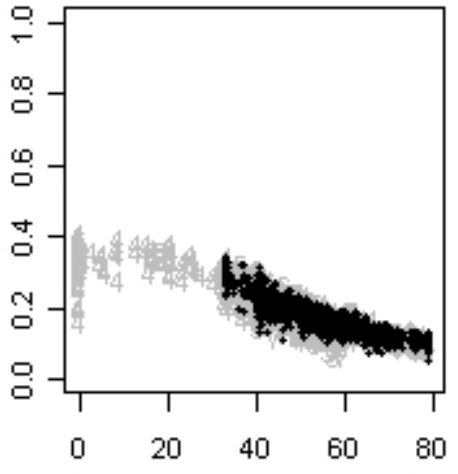

80

Figure 16. Passage probabilities of juvenile steelhead predicted by continuous covariates at McNary Dam through the spillway, turbines, and the juvenile bypass system in relation to total discharge and percent spill during day (grey symbols) and night (black symbols) photoperiods, 2004-09. Numbers used as plot symbols represent year of study, for example, 4=2004). 


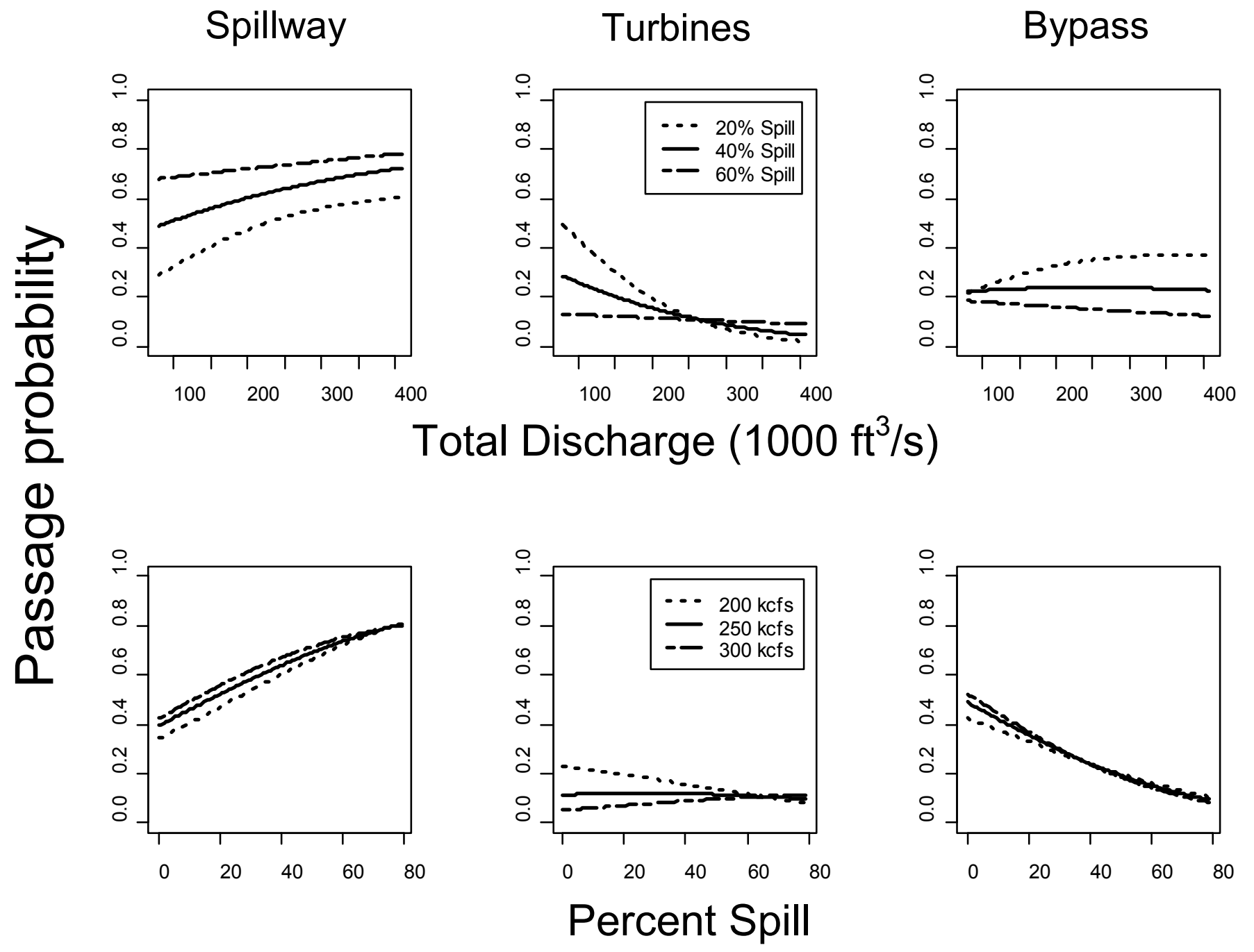

Figure 17. Passage probabilities of juvenile steelhead related to total discharge and percent spill at McNary Dam through the spillway, turbines, and the juvenile bypass system, 2004-09. Other covariates in the model were held constant at their mean value to examine the relationship to the variable of interest. 


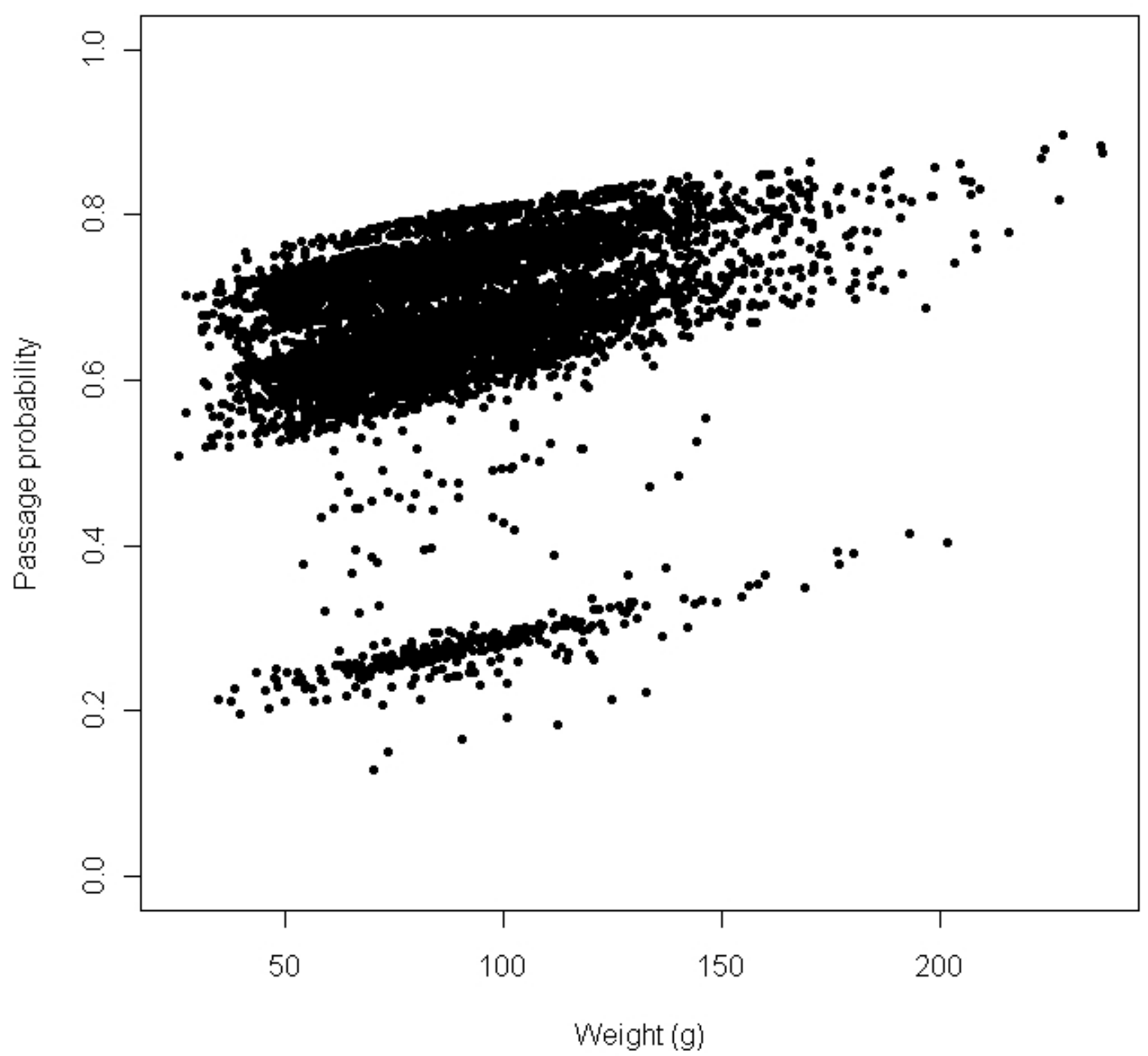

Figure 18. Passage probabilities of juvenile steelhead predicted by continuous covariates at McNary Dam through the spillway in relation to fish weight, 2004-09. 

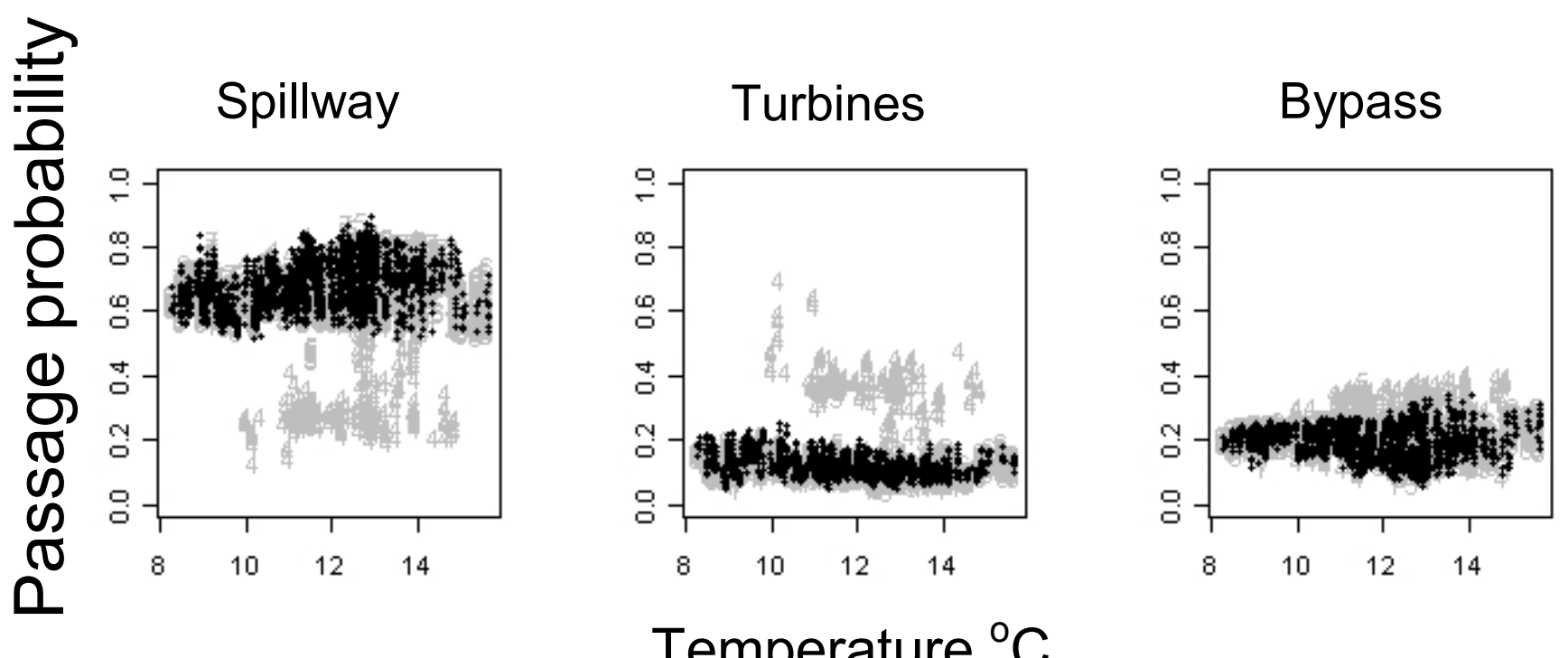

\section{Temperature ${ }^{\circ} \mathrm{C}$}

Figure 19. Passage probabilities of juvenile steelhead predicted by continuous covariates at McNary Dam through the spillway, turbines, and the juvenile bypass system in relation to water temperature during day (grey symbols) and night (black symbols) photoperiods, 2004-09. Numbers used as plot symbols represent year of study, e.g., 4=2004). 
Table 14. Slope (Beta) coefficients, standard error, and 95-percent confidence limits of model parameters of passage probabilities for juvenile steelhead passing McNary Dam, 2004-09.

[CL, confidence limit]

\begin{tabular}{lcccc}
\hline \multicolumn{1}{c}{ Variable } & \multicolumn{1}{c}{ Beta } & $\begin{array}{c}\text { Standard } \\
\text { error }\end{array}$ & Lower CL & Upper CL \\
\hline \multicolumn{2}{c}{ Spillway } \\
& 1.628 & 0.069 & 1.493 & 1.763 \\
\hline Photoperiod (dark) & 0.256 & 0.089 & 0.081 & 0.431 \\
Photoperiod (light) & 0.192 & 0.066 & 0.062 & 0.321 \\
\%Spill & 0.308 & 0.049 & 0.212 & 0.405 \\
Discharge & 0.178 & 0.029 & 0.120 & 0.236 \\
Weight & -0.080 & 0.031 & -0.140 & -0.020 \\
Temperature & -0.244 & 0.063 & -0.369 & -0.120 \\
Discharge×\%Spill & Bypass & & & \\
\hline & 0.947 & 0.074 & 0.801 & 1.093 \\
\hline Photoperiod (dark) & -0.648 & 0.102 & -0.848 & -0.449 \\
Photoperiod (light) & -0.461 & 0.077 & -0.611 & -0.310 \\
\%Spill & 0.323 & 0.051 & 0.222 & 0.423 \\
Discharge & -0.290 & 0.051 & -0.390 & -0.190 \\
Discharge×\%Spill & & & & \\
\hline
\end{tabular}




\section{Survival Analysis}

For survival analyses of juvenile steelhead passing through turbines and the spillway, the best model for $p$ and $\lambda$ was the model that expressed both with a year effect (table 15). The best model for $p$ and $\lambda$ for fish that passed through the juvenile bypass system or were released in the tailrace of McNary Dam expressed $\lambda$ constant across years and $p$ with a year effect.

Forebay survival was best explained by the model that included photoperiod, percent spill, total discharge, and water temperature (table 16). Survival was higher during the night than during day for all covariates and the difference in survival between day and night was greater when the TSWs were not installed compared to when they were (fig. 20). Survival was negatively related to water temperature and predicted survival ranged from 0.912 to 0.995 when other covariates were held constant at their mean value (fig. 20). Survival of fish in the forebay was positively related to discharge (fig. 20). Survival predicted by discharge ranged from 0.839 to 0.996 . Survival through the forebay predicted by percent spill ranged from 0.961 to 0.998 . Survival was fairly constant in relation to water temperature and percent spill when TSWs were installed (during the 2007 through 2009 migration periods). When TSWs were not installed (during the 2004 through 2006 migration periods), survival increased substantially as discharge increased (fig. 20).

The best-fit model of survival for juvenile steelhead passing through turbines included photoperiod and total discharge (table 17). Survival was positively related to total discharge and was more than 15 percent lower during the day (0.883) than the night $(0.728)$, as predicted by covariates while holding other model covariates at their mean value (fig. 21, table 18). Group covariates for photoperiod explained a significant amount of variation and had an AIC value more than 6 units less than the model that did not include photoperiod (table 17). Head was a variable that was highly correlated with total discharge and caused high variance inflation factors when included along with discharge, so it was not included in the same model. However, head was substituted into models for discharge and fit the data better based on AIC values being almost 4 AIC units lower. Predicted survival ranged from 0.477 when head was high to 0.966 when head was at low levels while holding other model covariates at their mean.

For juvenile steelhead that passed McNary Dam through the juvenile bypass system, the best model for survival included temperature and total discharge (table 19). Survival was positively related to discharge but negatively related to water temperature (fig. 22, table 18). In relation to water temperature, predicted survival ranged from about 0.84 to 0.98 as temperature ranged from 8.3 to $15.6^{\circ} \mathrm{C}$ while holding other model covariates at their mean value. Furthermore, survival predicted from total discharge ranged from about 0.88 to 0.98 where discharge ranged from 79 to $407 \mathrm{kcfs}$.

The best fit model for fish passing through the spillway included spillway passage location, fish weight, temperature, discharge, and percent spill (table 20). Survival was positively related to discharge, percent spill, and fish weight, but negatively related to temperature (table 18, figs. 23 and 24). The strongest relationship for survival was with percent spill. Survival increased as percent spill increased and leveled off when percent spill approached 80 percent. Survival predicted by percent spill ranged from 0.870 when percent spill was low to 0.987 when it was high while holding other model covariates at their mean value. Passage location was an important variable describing variation in survival only 
because of the 'Unknown' category for fish that could not be assigned to a particular section of the spillway. We were able to determine that these fish passed the spillway, but we could not definitively assign them to the north or south passage areas. This group of 'Unknown' fish had lower survival than fish that were assigned as either passing the north or south portion of the spillway. Survival estimates between fish that passed the north versus the south spillway were similar. Because fish weight described a significant amount of variation, other similar variables were added to the models such as tag weight and tag burden (tag weight/fish weight). The best model regarding model fit included tag weight and fish weight in the same model. This improved model fit by 8 AIC units compared to either model that had only fish weight or tag burden.

For juvenile steelhead released in the tailrace of McNary Dam, survival was best explained by total discharge, percent spill, and photoperiod (table 21). Survival was higher during the day than the night photoperiod, but all years were not represented regarding fish released during the night photoperiod; therefore, this result could be confounded. Survival was positively related to discharge, but negatively related to percent spill (table 18, fig. 25). The interaction between percent spill and discharge was not significant at the $\alpha$ level of 0.05 , but was significant at the $\alpha$ level of 0.10 indicating that there may be an interaction that could explain the negative relation between survival and percent spill. Including this interaction showed that survival of fish released in the tailrace had higher survival when discharge was high and percent spill was low (figs. 26 and 27). When percent spill was greater than about 50 percent, survival was similar at the various levels of discharge we examined. Release location also was tested to determine if there was a difference in survival among fish released in the north, middle, or south portion of the channel in the tailrace and there was no statistical difference.

A year factor was added to the best model for survival of juvenile steelhead for each route in order to determine if it would explain any additional variation in survival. Adding a year effect did not explain additional variation of significance for fish that went through turbines or for fish released in the tailrace, but did explain additional variation for fish that passed through the juvenile bypass system, spillway, or that were migrating through the forebay. A factor indicating whether a TSW was installed during a particular year also was included in place of year when survival through the spillway was examined and did not explain additional variation in survival for juvenile steelhead passing through the spillway, but did explain additional variation of survival in the forebay (fig. 20). Survival in the forebay was about 0.02 higher during years when the TSWs were installed during the day, and about 0.01 higher at night when other model covariates were held constant at their mean value. 
Table 15. Model selection results based on varying $\lambda$ and $p$ with respect to year for juvenile steelhead at McNary Dam, 2004-09.

[The best-fit models are indicated in bold. The best-fit model from assessing $\lambda$ was then used to assess the best-fit model for p. $\varnothing$, the probability of survival in the forebay or from dam passage through a route to the first downstream detection array; $p$, the probability of being detected at the first downstream detection array given the individual survived; $\lambda$, the joint probability of surviving and being detected from the first downstream array to the next downstream array; y, year]

\begin{tabular}{|c|c|c|c|c|c|}
\hline & Model & $\begin{array}{c}\text { Number of } \\
\text { parameters }\end{array}$ & $\mathrm{AIC} c$ & $\Delta \mathrm{AIC} c$ & Deviance \\
\hline \multicolumn{6}{|c|}{ Forebay } \\
\hline 1 & $\theta(\mathrm{y}) p(\mathrm{y}) \lambda(\mathrm{y})$ & 15 & $3,505.83$ & 0.00 & 0.000 \\
\hline 2 & $\varnothing(\mathrm{y}) p(\mathrm{y}) \lambda$ & 11 & $3,540.09$ & 34.27 & 42.295 \\
\hline 3 & $\varnothing(\mathrm{y}) p \lambda(\mathrm{y})$ & 11 & $6,654.07$ & 148.24 & 156.268 \\
\hline \multicolumn{6}{|c|}{ Turbine } \\
\hline 1 & $\sigma(\mathrm{y}) p(\mathrm{y}) \lambda(\mathrm{y})$ & 18 & 763.31 & 0.00 & 0.000 \\
\hline 2 & $\varnothing(\mathrm{y}) p(\mathrm{y}) \lambda$ & 13 & 769.89 & 6.58 & 17.188 \\
\hline 3 & $\varnothing(\mathrm{y}) p \lambda(\mathrm{y})$ & 13 & 813.86 & 50.55 & 61.158 \\
\hline \multicolumn{6}{|c|}{ Bypass } \\
\hline 2 & $\theta(\mathrm{y}) p(\mathrm{y}) \lambda$ & 13 & $2,505.75$ & 0.00 & 6.928 \\
\hline 1 & $\varnothing(\mathrm{y}) p(\mathrm{y}) \lambda(\mathrm{y})$ & 18 & $2,508.96$ & 3.21 & 0.000 \\
\hline 3 & $\varnothing(\mathrm{y}) p \lambda$ & 8 & $2,727.07$ & 221.32 & 238.342 \\
\hline \multicolumn{6}{|c|}{ Spillway } \\
\hline 1 & $\sigma(\mathrm{y}) p(\mathrm{y}) \lambda(\mathrm{y})$ & 18 & $7,474.63$ & 0.00 & 0.000 \\
\hline 2 & $\varnothing(\mathrm{y}) p(\mathrm{y}) \lambda$ & 13 & $7,498.20$ & 23.57 & 33.614 \\
\hline 3 & $\varnothing(\mathrm{y}) p \lambda(\mathrm{y})$ & 13 & $8,339.33$ & 864.69 & 874.737 \\
\hline \multicolumn{6}{|c|}{ Tailrace Releases } \\
\hline 2 & $\theta(\mathrm{y}) p(\mathrm{y}) \lambda$ & 7 & $3,788.15$ & 0.00 & 1.269 \\
\hline 1 & $\varnothing(\mathrm{y}) p(\mathrm{y}) \lambda(\mathrm{y})$ & 9 & $3,790.90$ & 2.75 & 0.000 \\
\hline 3 & $\varnothing(\mathrm{y}) p \lambda$ & 5 & $4,121.16$ & 333.01 & 338.290 \\
\hline
\end{tabular}




\section{TSW not installed}
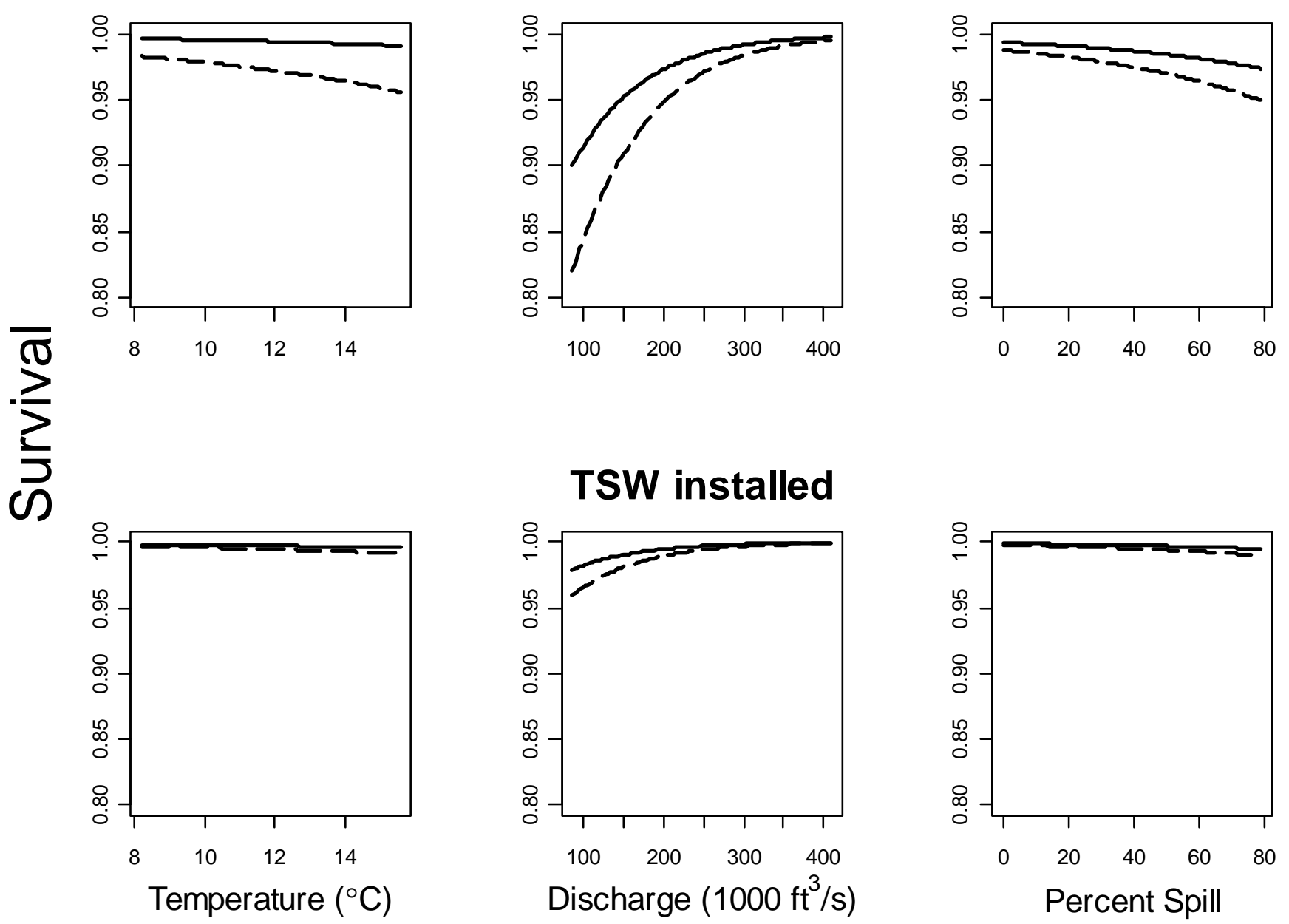

\section{TSW installed}
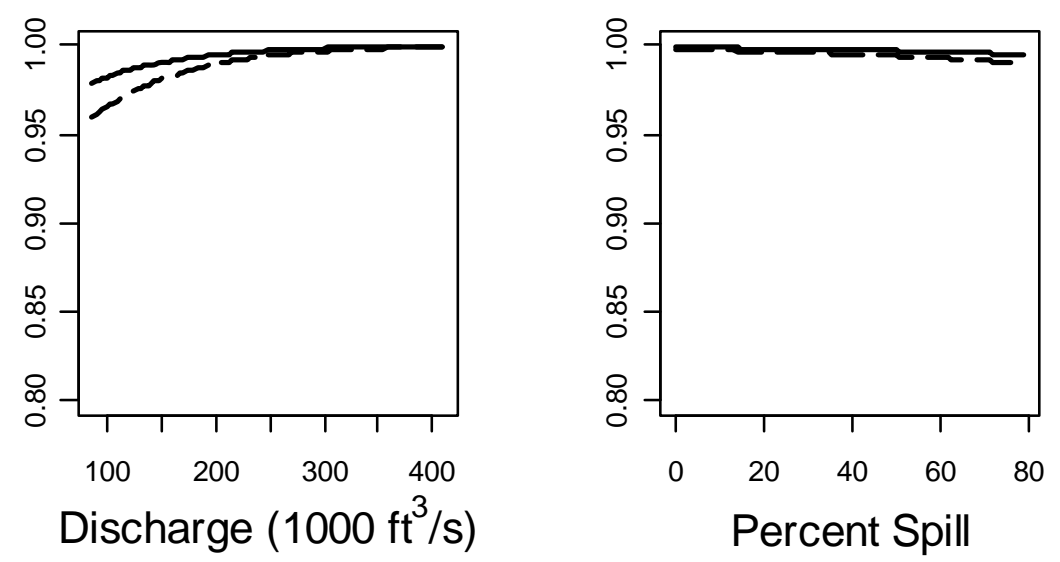

Figure 20. Survival of juvenile steelhead migrating through the forebay of McNary Dam during day (dashed line) and night (solid line) time photoperiods in relation to water temperature, total discharge, and percent spill. The TSWs were not installed during 2004-06. The TSWs were installed during 2007-09. 
Table 16. Model selection for fish that passed through the forebay at McNary Dam relating survival $(\varnothing)$ as a function of environmental variables using the fixed structure of the best model for $p$ and $\lambda$ parameters for juvenile steelhead, 2004-09.

\begin{tabular}{|c|c|c|c|c|c|}
\hline Model & $\begin{array}{l}\text { Number of } \\
\text { parameters }\end{array}$ & $\mathrm{AIC} c$ & $\begin{array}{l}\Delta \mathrm{AIC} c \\
\text { (group) }\end{array}$ & $\begin{array}{c}\Delta \mathrm{AIC} c \\
\text { (overall) }\end{array}$ & Deviance \\
\hline Intercept only & 11 & $3,564.32$ & 31.17 & 33.60 & 90.173 \\
\hline Discharge & 12 & $3,541.70$ & 8.55 & 10.98 & $3,517.664$ \\
\hline$\%$ Spill & 12 & $3,564.25$ & 31.10 & 33.53 & $3,540.214$ \\
\hline Temperature & 12 & $3,565.63$ & 32.48 & 34.91 & $3,541.593$ \\
\hline Weight & 12 & $3,566.17$ & 33.02 & 35.44 & $3,542.127$ \\
\hline Temperature + Discharge & 13 & $3,536.59$ & 3.43 & 5.86 & $3,510.537$ \\
\hline$\%$ Spill + Discharge & 13 & $3,541.10$ & 7.94 & 10.37 & $3,515.048$ \\
\hline Temperature $+\%$ Spill & 13 & $3,565.26$ & 32.11 & 34.54 & $3,539.217$ \\
\hline Temperature + Discharge $+\%$ Spill & 14 & $3,533.83$ & 0.68 & 3.10 & $3,505.775$ \\
\hline Weight + Temperature + Discharge & 14 & $3,537.17$ & 4.02 & 6.44 & $3,509.116$ \\
\hline$\%$ Spill + Discharge $+\%$ Spill $\times$ Discharge & 14 & $3,539.41$ & 6.26 & 8.68 & $3,511.354$ \\
\hline Weight $+\%$ Spill + Discharge & 14 & $3,541.81$ & 8.66 & 11.09 & $3,513.758$ \\
\hline Weight + Temperature $+\%$ Spill & 14 & $3,566.74$ & 33.59 & 36.01 & $3,538.682$ \\
\hline Temperature + Discharge $+\%$ Spill $+\%$ Spill $\times$ Discharge & 15 & $3,533.15$ & 0.00 & 2.43 & $3,503.089$ \\
\hline Weight + Temperature + Discharge $+\%$ Spill & 15 & $3,534.11$ & 0.96 & 3.38 & $3,504.044$ \\
\hline Weight $+\%$ Spill + Discharge $+\%$ Spill $\times$ Discharge & 15 & $3,540.26$ & 7.11 & 9.53 & $3,510.194$ \\
\hline Weight + Temperature + Discharge $+\%$ Spill $+\%$ Spill $\times$ Discharge & 16 & $3,533.63$ & 0.48 & 2.91 & $3,501.562$ \\
\hline
\end{tabular}


Table 16 (continued). Model selection for fish that passed through the forebay at McNary Dam relating survival $(\boldsymbol{\theta})$ as a function of environmental variables and group covariates for photoperiod using the fixed structure of the best model for $\boldsymbol{p}$ and $\lambda$ parameters for juvenile steelhead, 2004-09.

[The best-fit model is indicated in bold]

\begin{tabular}{|c|c|c|c|c|c|}
\hline Model & $\begin{array}{l}\text { Number of } \\
\text { parameters }\end{array}$ & $\mathrm{AIC} c$ & $\begin{array}{l}\Delta \mathrm{AIC} c \\
\text { (group) }\end{array}$ & $\begin{array}{c}\Delta \mathrm{AIC} c \\
\text { (overall) }\end{array}$ & Deviance \\
\hline Photoperiod & 12 & $3,557.07$ & 26.34 & 26.34 & 80.913 \\
\hline Photoperiod + Discharge & 13 & $3,540.59$ & 9.87 & 9.87 & $3,514.546$ \\
\hline Photoperiod $+\%$ Spill & 13 & $3,556.09$ & 25.36 & 25.36 & $3,530.037$ \\
\hline Weight + Photoperiod & 13 & $3,558.45$ & 27.72 & 27.72 & $3,532.402$ \\
\hline Temperature + Photoperiod & 13 & $3,558.51$ & 27.78 & 27.78 & $3,532.459$ \\
\hline Photoperiod $+\%$ Spill + Discharge & 14 & $3,538.38$ & 7.65 & 7.65 & $3,510.322$ \\
\hline Weight + Photoperiod + Discharge & 14 & $3,541.34$ & 10.61 & 10.61 & $3,513.285$ \\
\hline Weight + Photoperiod $+\%$ Spill & 14 & $3,557.58$ & 26.85 & 26.85 & $3,529.523$ \\
\hline Weight + Temperature + Photoperiod & 14 & $3,559.77$ & 29.05 & 29.05 & $3,531.718$ \\
\hline Photoperiod $+\%$ Spill + Discharge + Temperature & 15 & $3,531.56$ & 0.83 & 0.83 & $3,501.497$ \\
\hline Photoperiod $+\%$ Spill + Discharge $+\%$ Spill $\times$ Discharge & 15 & $3,536.45$ & 5.72 & 5.72 & $3,506.388$ \\
\hline Weight + Photoperiod $+\%$ Spill + Discharge & 15 & $3,539.03$ & 8.30 & 8.30 & $3,508.966$ \\
\hline Photoperiod $+\%$ Spill + Discharge + Temperature $+\%$ Spill $\times$ Discharge & 16 & $3,530.73$ & 0.00 & 0.00 & $3,498.655$ \\
\hline Weight + Photoperiod $+\%$ Spill + Discharge + Temperature & 16 & $3,531.92$ & 1.19 & 1.19 & $3,499.847$ \\
\hline Weight + Photoperiod $+\%$ Spill + Discharge $+\%$ Spill $\times$ Discharge & 16 & $3,537.17$ & 6.45 & 6.45 & $3,505.103$ \\
\hline Weight + Photoperiod $+\%$ Spill + Discharge + Temperature $+\%$ Spill $\times$ Discharge & 17 & $3,531.22$ & 0.50 & 0.50 & $3,497.143$ \\
\hline
\end{tabular}


Table 17. Model selection for fish that passed through turbines at McNary Dam relating survival $(\varnothing)$ as a function of environmental variables using the fixed structure of the best model for $p$ and $\lambda$ parameters for juvenile steelhead, 2004-09.

\begin{tabular}{|c|c|c|c|c|c|}
\hline Model & $\begin{array}{l}\text { Number of } \\
\text { parameters }\end{array}$ & $\mathrm{AIC} c$ & $\begin{array}{l}\Delta \mathrm{AIC} c \\
\text { (group) }\end{array}$ & $\begin{array}{c}\Delta \mathrm{AIC} c \\
\text { (overall) }\end{array}$ & Deviance \\
\hline Intercept only & 13 & 757.54 & 4.96 & 11.54 & 68.793 \\
\hline Discharge & 14 & 752.58 & 0.00 & 6.58 & 723.796 \\
\hline$\%$ Spill & 14 & 757.46 & 4.87 & 11.45 & 728.667 \\
\hline Temperature & 14 & 759.28 & 6.69 & 13.28 & 730.491 \\
\hline Weight & 14 & 759.33 & 6.75 & 13.33 & 730.542 \\
\hline Temperature + Discharge & 15 & 754.54 & 1.96 & 8.54 & 723.642 \\
\hline$\%$ Spill + Discharge & 15 & 754.68 & 2.09 & 8.68 & 723.776 \\
\hline Temperature +\%Spill & 15 & 759.29 & 6.71 & 13.29 & 728.390 \\
\hline Weight + Temperature + Discharge & 16 & 755.80 & 3.22 & 9.80 & 722.775 \\
\hline Weight $+\%$ Spill + Discharge & 16 & 755.92 & 3.33 & 9.91 & 722.892 \\
\hline Temperature + Discharge $+\%$ Spill & 16 & 756.66 & 4.08 & 10.66 & 723.637 \\
\hline Weight + Temperature $+\%$ Spill & 16 & 760.79 & 8.20 & 14.79 & 727.763 \\
\hline Weight $+\%$ Spill + Discharge $+\%$ Spill $\times$ Discharge & 17 & 756.52 & 3.93 & 10.51 & 721.362 \\
\hline Temperature + Discharge $+\%$ Spill $+\%$ Spill $\times$ Discharge & 17 & 756.81 & 4.22 & 10.80 & 721.652 \\
\hline Weight + Temperature + Discharge $+\%$ Spill & 17 & 757.91 & 5.32 & 11.91 & 722.754 \\
\hline Weight + Temperature + Discharge $+\%$ Spill $+\%$ Spill $\times$ Discharge & 18 & 758.12 & 5.54 & 12.12 & 720.826 \\
\hline
\end{tabular}


Table 17 (continued). Model selection for fish that passed through turbines at McNary Dam relating survival $(\boldsymbol{o})$ as a function of environmental variables and group covariates for photoperiod using the fixed structure of the best model for $\boldsymbol{p}$ and $\lambda$ parameters for juvenile steelhead, 2004-09.

[The best-fit model is indicated in bold]

\begin{tabular}{|c|c|c|c|c|c|}
\hline Model & $\begin{array}{l}\text { Number of } \\
\text { parameters }\end{array}$ & $\mathrm{AIC} c$ & $\begin{array}{c}\Delta \mathrm{AIC} c \\
\text { (group) }\end{array}$ & $\begin{array}{c}\Delta \mathrm{AICc} \\
\text { (overall) }\end{array}$ & Deviance \\
\hline Photoperiod & 14 & 753.07 & 7.07 & 7.07 & 62.218 \\
\hline Photoperiod + Discharge & 15 & 746.80 & 0.79 & 0.79 & 715.894 \\
\hline Photoperiod + Temperature & 15 & 754.07 & 8.06 & 8.06 & 723.164 \\
\hline Photoperiod $+\%$ Spill & 15 & 754.82 & 8.82 & 8.82 & 723.921 \\
\hline Photoperiod + Weight & 15 & 754.90 & 8.90 & 8.90 & 724.002 \\
\hline Photoperiod $+\%$ Spill + Discharge & 16 & 746.00 & 0.00 & 0.00 & 712.978 \\
\hline Photoperiod + Weight + Discharge & 16 & 748.10 & 2.10 & 2.10 & 715.075 \\
\hline Photoperiod + Weight + Temperature & 16 & 755.89 & 9.88 & 9.88 & 722.862 \\
\hline Photoperiod + Weight $+\%$ Spill & 16 & 756.53 & 10.53 & 10.53 & 723.510 \\
\hline Photoperiod $+\%$ Spill + Discharge $+\%$ Spill $\times$ Discharge & 17 & 746.01 & 0.01 & 0.01 & 710.855 \\
\hline Photoperiod + Weight $+\%$ Spill + Discharge & 17 & 747.69 & 1.69 & 1.69 & 712.536 \\
\hline Photoperiod $+\%$ Spill + Discharge + Temperature & 17 & 748.09 & 2.09 & 2.09 & 712.933 \\
\hline Photoperiod + Weight $+\%$ Spill + Discharge $+\%$ Spill $\times$ Discharge & 18 & 747.77 & 1.77 & 1.77 & 710.475 \\
\hline Photoperiod $+\%$ Spill + Discharge + Temperature $+\%$ Spill $\times$ Discharge & 18 & 747.82 & 1.81 & 1.81 & 710.523 \\
\hline Photoperiod + Weight $+\%$ Spill + Discharge + Temperature & 18 & 749.78 & 3.78 & 3.78 & 712.490 \\
\hline Photoperiod + Weight $+\%$ Spill + Discharge + Temperature $+\%$ Spill $\times$ Discharge & 19 & 749.57 & 3.57 & 3.57 & 710.130 \\
\hline
\end{tabular}


Table 17 (continued). Model selection for fish that passed through turbines at McNary Dam relating survival $(\boldsymbol{\theta})$ as a function of environmental variables and group covariates for turbine passage location (that is, north, south) using the fixed structure of the best model for $\boldsymbol{p}$ and $\lambda$ parameters for juvenile steelhead, 2004-09.

\begin{tabular}{|c|c|c|c|c|c|}
\hline Model & $\begin{array}{l}\text { Number of } \\
\text { parameters }\end{array}$ & $\mathrm{AIC}_{c}$ & $\begin{array}{l}\Delta \mathrm{AlC} c \\
\text { (group) }\end{array}$ & $\begin{array}{c}\Delta \mathrm{AIC} c \\
\text { (overall) }\end{array}$ & Deviance \\
\hline TurbPassLoc & 14 & 757.55 & 3.18 & 11.55 & 66.697 \\
\hline TurbPassLoc + Discharge & 15 & 754.38 & 0.00 & 8.37 & 723.475 \\
\hline TurbPassLoc $+\%$ Spill & 15 & 758.25 & 3.87 & 12.25 & 727.347 \\
\hline TurbPassLoc + Weight & 15 & 759.21 & 4.84 & 13.21 & 728.312 \\
\hline TurbPassLoc + Temperature & 15 & 759.22 & 4.84 & 13.22 & 728.315 \\
\hline TurbPassLoc + Weight + Discharge & 16 & 755.59 & 1.21 & 9.59 & 722.563 \\
\hline TurbPassLoc + Temperature + Discharge & 16 & 756.43 & 2.05 & 10.42 & 723.402 \\
\hline TurbPassLoc $+\%$ Spill + Discharge & 16 & 756.49 & 2.11 & 10.48 & 723.463 \\
\hline TurbPassLoc + Weight $+\%$ Spill & 16 & 759.68 & 5.31 & 13.68 & 726.660 \\
\hline TurbPassLoc + Temperature $+\%$ Spill & 16 & 760.01 & 5.63 & 14.01 & 726.985 \\
\hline TurbPassLoc $+\%$ Spill + Discharge $+\%$ Spill $\times$ Discharge & 17 & 756.71 & 2.33 & 10.70 & 721.551 \\
\hline TurbPassLoc + Weight + Temperature + Discharge & 17 & 757.64 & 3.26 & 11.64 & 722.485 \\
\hline TurbPassLoc + Weight $+\%$ Spill + Discharge & 17 & 757.68 & 3.31 & 11.68 & 722.529 \\
\hline TurbPassLoc + Temperature + Discharge $+\%$ Spill & 17 & 758.55 & 4.18 & 12.55 & 723.398 \\
\hline TurbPassLoc + Weight + Temperature $+\%$ Spill & 17 & 761.44 & 7.06 & 15.44 & 726.286 \\
\hline TurbPassLoc + Temperature + Discharge $+\%$ Spill $+\%$ Spill $\times$ Discharge & 18 & 758.49 & 4.11 & 12.49 & 721.197 \\
\hline TurbPassLoc + Weight + Temperature + Discharge $+\%$ Spill & 18 & 759.76 & 5.38 & 13.76 & 722.465 \\
\hline TurbPassLoc + Weight + Temperature + Discharge $+\%$ Spill $+\%$ Spill $\times$ Discharge & 19 & 759.73 & 5.35 & 13.73 & 720.290 \\
\hline
\end{tabular}


Table 17 (continued). Model selection for fish that passed through turbines at McNary Dam relating survival $(\boldsymbol{o})$ as a function of environmental variables and group covariates for turbine passage location (that is, north, south) and photoperiod using the fixed structure of the best model for $\boldsymbol{p}$ and $\lambda$ parameters for juvenile steelhead, 2004-09.

\begin{tabular}{|c|c|c|c|c|c|}
\hline Model & $\begin{array}{l}\text { Number of } \\
\text { parameters }\end{array}$ & $\mathrm{AIC} c$ & $\begin{array}{c}\Delta \mathrm{AlC} c \\
\text { (group) }\end{array}$ & $\begin{array}{c}\Delta \mathrm{AIC} c \\
\text { (overall) }\end{array}$ & Deviance \\
\hline TurbPassLoc + Photoperiod & 15 & 752.33 & 5.53 & 6.33 & 59.363 \\
\hline TurbPassLoc + Photoperiod + Discharge & 16 & 748.46 & 1.66 & 2.46 & 715.439 \\
\hline TurbPassLoc + Photoperiod + Temperature & 16 & 753.21 & 6.41 & 7.21 & 720.185 \\
\hline TurbPassLoc + Photoperiod + Temperature & 16 & 753.21 & 6.41 & 7.21 & 720.185 \\
\hline TurbPassLoc + Photoperiod + Weight & 16 & 754.00 & 7.20 & 8.00 & 720.979 \\
\hline TurbPassLoc + Photoperiod $+\%$ Spill & 16 & 754.45 & 7.65 & 8.45 & 721.428 \\
\hline TurbPassLoc + Photoperiod $+\%$ Spill + Discharge & 17 & 747.31 & 0.51 & 1.31 & 712.155 \\
\hline TurbPassLoc + Photoperiod + Weight + Discharge & 17 & 749.69 & 2.89 & 3.69 & 714.539 \\
\hline TurbPassLoc + Photoperiod + Weight + Temperature & 17 & 754.89 & 8.09 & 8.89 & 719.734 \\
\hline TurbPassLoc + Photoperiod + Weight $+\%$ Spill & 17 & 756.11 & 9.31 & 10.11 & 720.954 \\
\hline TurbPassLoc + Photoperiod $+\%$ Spill + Discharge $+\%$ Spill $\times$ Discharge & 18 & 746.80 & 0.00 & 0.80 & 709.508 \\
\hline TurbPassLoc + Photoperiod + Weight $+\%$ Spill + Discharge & 18 & 748.93 & 2.13 & 2.93 & 711.639 \\
\hline TurbPassLoc + Photoperiod $+\%$ Spill + Discharge + Temperature & 18 & 749.45 & 2.65 & 3.44 & 712.154 \\
\hline TurbPassLoc + Photoperiod + Weight $+\%$ Spill + Discharge $+\%$ Spill $\times$ Discharge & 19 & 748.45 & 1.65 & 2.45 & 709.015 \\
\hline TurbPassLoc + Photoperiod $+\%$ Spill + Discharge + Temperature $+\%$ Spill $\times$ Discharge & 19 & 748.80 & 1.99 & 2.79 & 709.356 \\
\hline TurbPassLoc + Photoperiod + Weight $+\%$ Spill + Discharge + Temperature & 19 & 751.08 & 4.28 & 5.07 & 711.638 \\
\hline TurbPassLoc + Photoperiod + Weight $+\%$ Spill + Discharge + Temperature $+\%$ Spill $\times$ Discharge & 20 & 750.44 & 3.64 & 4.44 & 708.848 \\
\hline
\end{tabular}



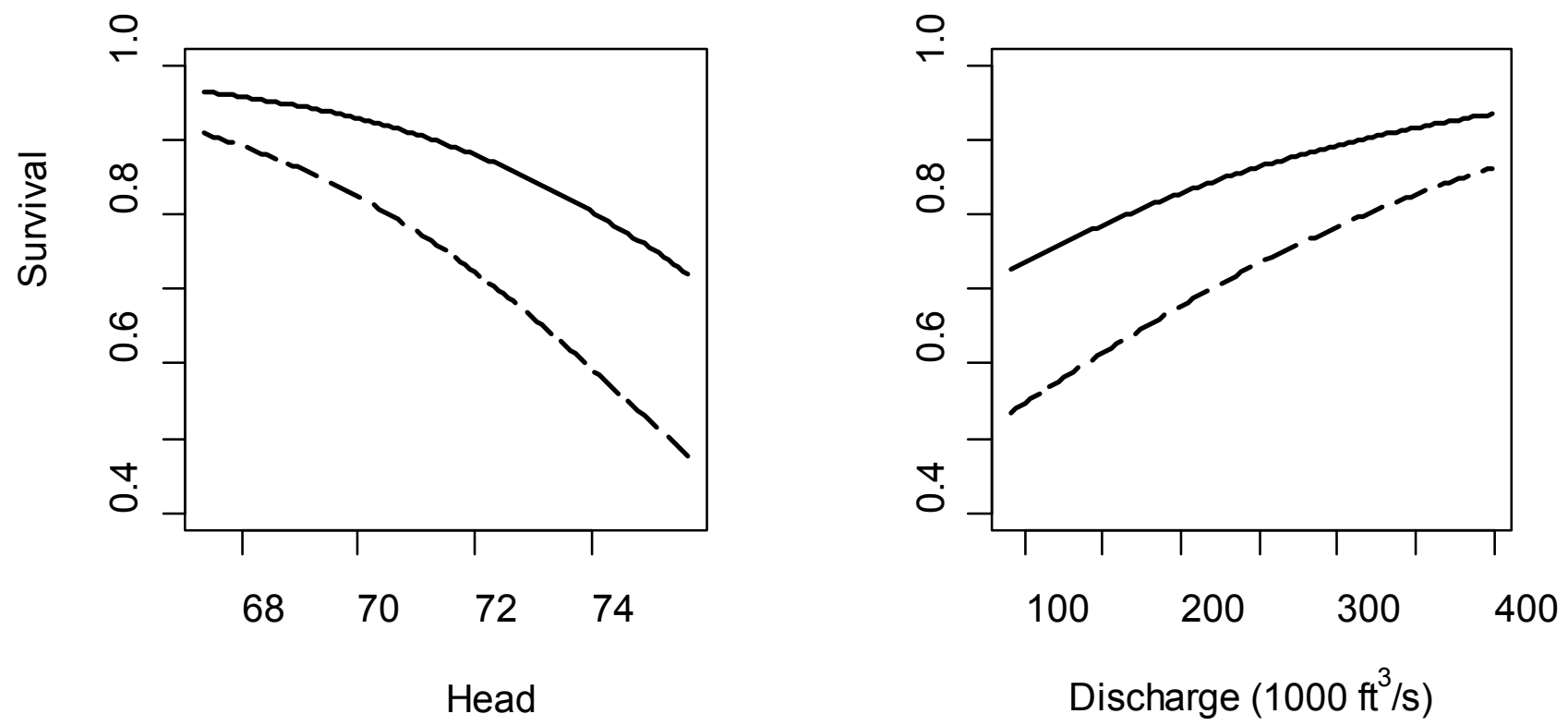

Figure 21. Survival of juvenile steelhead that passed through turbines at McNary Dam in relation to head (forebay elevation - tailrace elevation) and total discharge during day (dashed line) and night (solid line) photoperiods, 2004-09. 
Table 18. Slope (Beta) coefficients, standard error, and 95-percent confidence limits of model parameters for juvenile steelhead passing McNary Dam, 2004-09.

[CL, confidence limit]

\begin{tabular}{|c|c|c|c|c|}
\hline Variable & Beta & $\begin{array}{c}\text { Standard } \\
\text { error }\end{array}$ & Lower CL & Upper CL \\
\hline \multicolumn{5}{|c|}{ Forebay } \\
\hline Intercept (TSW not installed) & 2.731 & 1.302 & 0.179 & 5.283 \\
\hline TSW (installed) & 1.663 & 0.389 & 0.901 & 2.425 \\
\hline$\%$ Spill & -0.015 & 0.007 & -0.029 & -0.0005 \\
\hline Discharge & 0.012 & 0.002 & 0.007 & 0.017 \\
\hline Temperature & -0.131 & 0.115 & -0.357 & 0.094 \\
\hline \multicolumn{5}{|c|}{ Turbine } \\
\hline Intercept & 22.851 & 6.640 & 9.837 & 35.866 \\
\hline Photoperiod (light) & -1.037 & 0.342 & -1.708 & -0.366 \\
\hline Head & -0.290 & 0.090 & -0.466 & -0.113 \\
\hline \multicolumn{5}{|c|}{ Bypass } \\
\hline Intercept & 5.257 & 1.098 & 3.105 & 7.408 \\
\hline Temperature & -0.318 & 0.090 & -0.494 & -0.142 \\
\hline Discharge & 0.006 & 0.002 & 0.002 & 0.009 \\
\hline \multicolumn{5}{|c|}{ Spillway } \\
\hline Intercept & 3.562 & 0.918 & 1.762 & 5.361 \\
\hline SpillPassLoc (North) & 1.357 & 0.397 & 0.579 & 2.135 \\
\hline SpillPassLoc (South) & 1.471 & 0.392 & 0.702 & 2.240 \\
\hline Weight & 0.013 & 0.003 & 0.006 & 0.019 \\
\hline Tag Weight & -1.791 & 0.577 & -2.922 & -0.659 \\
\hline Temperature & -0.317 & 0.055 & -0.425 & -0.209 \\
\hline$\%$ Spill & 0.049 & 0.012 & 0.026 & 0.073 \\
\hline Discharge & 0.004 & 0.002 & 0.001 & 0.008 \\
\hline \multicolumn{5}{|c|}{ Tailrace Releases } \\
\hline Intercept & 2.394 & 0.493 & 1.428 & 3.360 \\
\hline Photoperiod (light) & 0.438 & 0.249 & -0.050 & 0.926 \\
\hline$\%$ Spill & -0.015 & 0.007 & -0.029 & -0.001 \\
\hline Discharge & 0.003 & 0.002 & 0.000 & 0.007 \\
\hline
\end{tabular}


Table 19. Model selection for fish that passed through the juvenile bypass system at McNary Dam relating survival (ø) as a function of environmental variables using the fixed structure of the best model for $p$ and $\lambda$ parameters for juvenile steelhead, 2004-09.

[The best-fit model is indicated in bold]

\begin{tabular}{|c|c|c|c|c|c|}
\hline Model & $\begin{array}{l}\text { Number of } \\
\text { parameters }\end{array}$ & $\mathrm{AIC} c$ & $\begin{array}{l}\Delta \mathrm{AIC} c \\
\text { (group) }\end{array}$ & $\begin{array}{c}\Delta \mathrm{AIC} c \\
\text { (overall) }\end{array}$ & Deviance \\
\hline Intercept only & 8 & $2,523.65$ & 14.31 & 14.31 & 73.312 \\
\hline Temperature & 9 & $2,516.87$ & 7.52 & 7.52 & $2,498.794$ \\
\hline Discharge & 9 & $2,521.33$ & 11.98 & 11.98 & $2,503.252$ \\
\hline$\%$ Spill & 9 & $2,521.43$ & 12.08 & 12.08 & $2,503.354$ \\
\hline Weight & 9 & $2,525.67$ & 16.32 & 16.32 & $2,507.592$ \\
\hline Temperature + Discharge & 10 & $2,509.86$ & 0.51 & 0.51 & $2,489.764$ \\
\hline Temperature $+\%$ Spill & 10 & $2,515.05$ & 5.70 & 5.70 & $2,494.955$ \\
\hline$\%$ Spill + Discharge & 10 & $2,521.93$ & 12.58 & 12.58 & $2,501.839$ \\
\hline Weight + Temperature + Discharge & 11 & $2,511.71$ & 2.37 & 2.37 & $2,489.602$ \\
\hline Temperature + Discharge $+\%$ Spill & 11 & $2,511.77$ & 2.43 & 2.43 & $2,489.661$ \\
\hline Weight + Temperature $+\%$ Spill & 11 & $2,517.06$ & 7.71 & 7.71 & $2,494.947$ \\
\hline$\%$ Spill + Discharge $+\%$ Spill $\times$ Discharge & 11 & $2,520.45$ & 11.11 & 11.11 & $2,498.340$ \\
\hline Weight $+\%$ Spill + Discharge & 11 & $2,523.91$ & 14.57 & 14.57 & $2,501.802$ \\
\hline Temperature + Discharge $+\%$ Spill $+\%$ Spill $\times$ Discharge & 12 & $2,509.35$ & 0.00 & 0.00 & $2,485.215$ \\
\hline Weight + Temperature + Discharge $+\%$ Spill & 12 & $2,513.65$ & 4.30 & 4.30 & $2,489.517$ \\
\hline Weight $+\%$ Spill + Discharge $+\%$ Spill $\times$ Discharge & 12 & $2,522.41$ & 13.07 & 13.07 & $2,498.281$ \\
\hline Weight + Temperature + Discharge $+\%$ Spill $+\%$ Spill $\times$ Discharge & 13 & $2,511.21$ & 1.86 & 1.86 & $2,485.052$ \\
\hline
\end{tabular}


Table 19 (continued). Model selection for fish that passed through the juvenile bypass system at McNary Dam relating survival ( $\boldsymbol{\theta})$ as a function of environmental variables and group covariates for photoperiod using the fixed structure of the best model for $\boldsymbol{p}$ and $\lambda$ parameters for juvenile steelhead, 2004-09.

\begin{tabular}{|c|c|c|c|c|c|}
\hline Model & $\begin{array}{l}\text { Number of } \\
\text { parameters }\end{array}$ & $\mathrm{AIC} c$ & $\begin{array}{l}\Delta \mathrm{AIC} c \\
\text { (group) }\end{array}$ & $\begin{array}{c}\Delta \mathrm{AIC} c \\
\text { (overall) }\end{array}$ & Deviance \\
\hline Photoperiod & 9 & $2,524.64$ & 13.34 & 15.30 & 72.287 \\
\hline Photoperiod + Temperature & 10 & $2,518.56$ & 7.25 & 9.21 & $2,498.463$ \\
\hline Photoperiod + Discharge & 10 & $2,522.05$ & 10.74 & 12.70 & $2,501.954$ \\
\hline Photoperiod $+\%$ Spill & 10 & $2,523.35$ & 12.04 & 14.00 & $2,503.255$ \\
\hline Photoperiod + Weight & 10 & $2,526.65$ & 15.34 & 17.30 & $2,506.559$ \\
\hline Photoperiod + Weight + Temperature & 11 & $2,520.57$ & 9.26 & 11.23 & $2,498.461$ \\
\hline Photoperiod $+\%$ Spill + Discharge & 11 & $2,523.54$ & 12.23 & 14.19 & $2,501.425$ \\
\hline Photoperiod + Weight + Discharge & 11 & $2,524.03$ & 12.72 & 14.68 & $2,501.919$ \\
\hline Photoperiod + Discharge + Temperature & 11 & $2,511.76$ & 0.45 & 2.41 & $2,489.643$ \\
\hline Photoperiod + Weight $+\%$ Spill & 11 & $2,525.37$ & 14.06 & 16.02 & $2,503.255$ \\
\hline Photoperiod $+\%$ Spill + Discharge + Temperature & 12 & $2,513.75$ & 2.44 & 4.40 & $2,489.613$ \\
\hline Photoperiod $+\%$ Spill + Discharge $+\%$ Spill $\times$ Discharge & 12 & $2,521.99$ & 10.68 & 12.64 & $2,497.854$ \\
\hline Photoperiod + Weight $+\%$ Spill + Discharge & 12 & $2,525.53$ & 14.22 & 16.18 & $2,501.393$ \\
\hline Photoperiod $+\%$ Spill + Discharge + Temperature $+\%$ Spill $\times$ Discharge & 13 & $2,511.31$ & 0.00 & 1.96 & $2,485.154$ \\
\hline Photoperiod + Weight $+\%$ Spill + Discharge + Temperature & 13 & $2,515.62$ & 4.32 & 6.28 & $2,489.469$ \\
\hline Photoperiod + Weight $+\%$ Spill + Discharge $+\%$ Spill $\times$ Discharge & 13 & $2,523.95$ & 12.64 & 14.60 & $2,497.797$ \\
\hline Photoperiod + Weight $+\%$ Spill + Discharge + Temperature $+\%$ Spill $\times$ Discharge & 14 & $2,513.17$ & 1.86 & 3.82 & $2,484.989$ \\
\hline
\end{tabular}



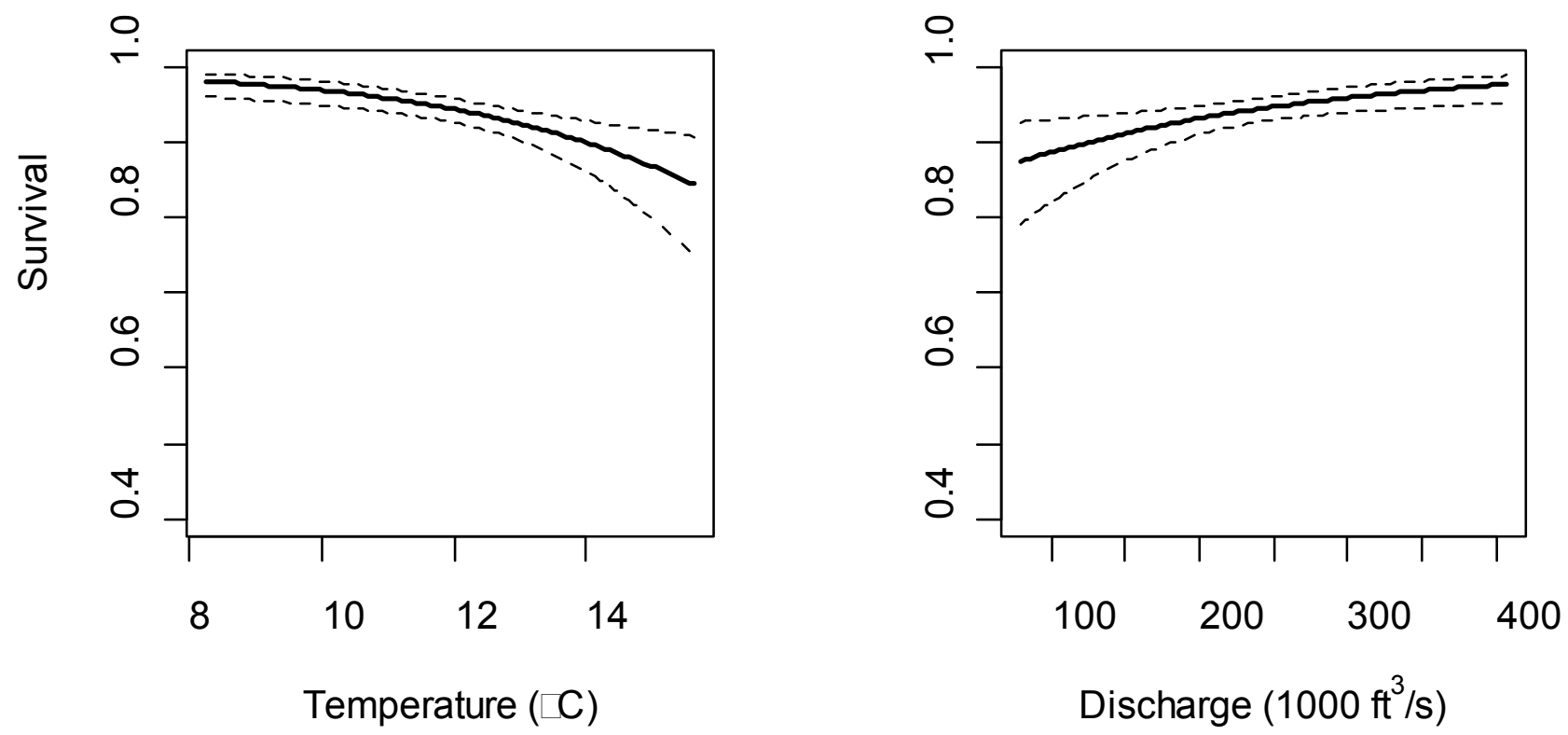

Figure 22. Survival of juvenile steelhead that passed through the juvenile bypass system at McNary Dam in relation to temperature and total discharge, 2004-09. The dashed lines represent the 95-percent confidence limits. Other covariates in the model were held constant at their mean value to examine the relationship to the variable of interest. 
Table 20. Model selection for fish that passed through the spillway at McNary Dam relating survival ( $\varnothing)$ as a function of environmental variables using the fixed structure of the best model for $p$ and $\lambda$ parameters for juvenile steelhead, 2004-09.

\begin{tabular}{|c|c|c|c|c|c|}
\hline Model & $\begin{array}{l}\text { Number of } \\
\text { parameters }\end{array}$ & $\mathrm{AlC} c$ & $\begin{array}{c}\Delta \mathrm{AIC} c \\
\text { (group) }\end{array}$ & $\begin{array}{c}\Delta \mathrm{AIC} c \\
\text { (overall) }\end{array}$ & Deviance \\
\hline Intercept only & 13 & $7,490.78$ & 44.22 & 46.09 & 178.848 \\
\hline Weight & 14 & $7,478.72$ & 32.16 & 34.03 & $7,450.663$ \\
\hline Temperature & 14 & $7,479.44$ & 32.88 & 34.75 & $7,451.380$ \\
\hline$\%$ Spill & 14 & $7,490.34$ & 43.78 & 45.65 & $7,462.283$ \\
\hline Discharge & 14 & $7,492.47$ & 45.91 & 47.78 & $7,464.408$ \\
\hline Temperature $+\%$ Spill & 15 & $7,468.95$ & 22.39 & 24.26 & $7,438.884$ \\
\hline Temperature + Discharge & 15 & $7,472.84$ & 26.28 & 28.16 & $7,442.778$ \\
\hline$\%$ Spill + Discharge & 15 & $7,492.21$ & 45.65 & 47.52 & $7,462.143$ \\
\hline Weight + Temperature + Discharge & 16 & $7,456.45$ & 9.89 & 11.76 & $7,424.372$ \\
\hline Weight + Temperature $+\%$ Spill & 16 & $7,458.22$ & 11.65 & 13.53 & $7,426.140$ \\
\hline Temperature + Discharge $+\%$ Spill & 16 & $7,462.00$ & 15.44 & 17.31 & $7,429.926$ \\
\hline Weight $+\%$ Spill + Discharge & 16 & $7,479.55$ & 32.99 & 34.87 & $7,447.477$ \\
\hline$\%$ Spill + Discharge $+\%$ Spill $\times$ Discharge & 16 & $7,489.73$ & 43.17 & 45.04 & $7,457.656$ \\
\hline Weight + Temperature + Discharge $+\%$ Spill & 17 & $7,447.74$ & 1.18 & 3.05 & $7,413.656$ \\
\hline Temperature + Discharge $+\%$ Spill $+\%$ Spill $\times$ Discharge & 17 & $7,460.91$ & 14.34 & 16.22 & $7,426.821$ \\
\hline Weight $+\%$ Spill + Discharge $+\%$ Spill $\times$ Discharge & 17 & $7,476.78$ & 30.22 & 32.10 & $7,442.697$ \\
\hline Weight + Temperature + Discharge $+\%$ Spill $+\%$ Spill $\times$ Discharge & 18 & $7,446.56$ & 0.00 & 1.87 & $7,410.466$ \\
\hline
\end{tabular}


Table 20 (continued). Model selection for fish that passed through the spillway at McNary Dam relating survival ( $\varnothing$ ) as a function of environmental variables and group covariates for photoperiod using the fixed structure of the best model for $p$ and $\lambda$ parameters for juvenile steelhead, 2004-09.

\begin{tabular}{|c|c|c|c|c|c|}
\hline Model & $\begin{array}{l}\text { Number of } \\
\text { parameters }\end{array}$ & $\mathrm{AIC} c$ & $\begin{array}{l}\Delta \mathrm{AIC} c \\
\text { (group) }\end{array}$ & $\begin{array}{c}\Delta \mathrm{AIC} c \\
\text { (overall) }\end{array}$ & Deviance \\
\hline Photoperiod & 14 & $7,492.11$ & 43.57 & 47.43 & 178.174 \\
\hline Photoperiod + Temperature & 15 & $7,480.17$ & 31.62 & 35.48 & $7,450.098$ \\
\hline Photoperiod $+\%$ Spill & 15 & $7,492.24$ & 43.70 & 47.55 & $7,462.173$ \\
\hline Photoperiod + Discharge & 15 & $7,493.49$ & 44.95 & 48.80 & $7,463.421$ \\
\hline Photoperiod + Weight & 15 & $7,480.69$ & 32.15 & 36.00 & $7,450.623$ \\
\hline Photoperiod + Weight + Temperature & 16 & $7,469.70$ & 21.16 & 25.01 & $7,437.624$ \\
\hline Photoperiod + Weight $+\%$ Spill & 16 & $7,480.45$ & 31.91 & 35.77 & $7,448.379$ \\
\hline Photoperiod + Weight + Discharge & 16 & $7,480.98$ & 32.43 & 36.29 & $7,448.899$ \\
\hline Photoperiod $+\%$ Spill + Discharge & 16 & $7,494.00$ & 45.46 & 49.32 & $7,461.929$ \\
\hline Photoperiod + Weight + Temperature + Discharge & 17 & $7,456.18$ & 7.63 & 11.49 & $7,422.090$ \\
\hline Photoperiod $+\%$ Spill + Discharge + Temperature & 17 & $7,463.49$ & 14.95 & 18.80 & $7,429.405$ \\
\hline Photoperiod + Weight $+\%$ Spill + Discharge & 17 & $7,481.56$ & 33.02 & 36.87 & $7,447.476$ \\
\hline Photoperiod $+\%$ Spill + Discharge $+\%$ Spill $\times$ Discharge & 17 & $7,491.55$ & 43.00 & 46.86 & $7,457.460$ \\
\hline Photoperiod + Weight $+\%$ Spill + Discharge + Temperature & 18 & $7,449.70$ & 1.16 & 5.01 & $7,413.603$ \\
\hline Photoperiod $+\%$ Spill + Discharge + Temperature $+\%$ Spill $\times$ Discharge & 18 & $7,462.46$ & 13.92 & 17.77 & $7,426.363$ \\
\hline Photoperiod + Weight $+\%$ Spill + Discharge $+\%$ Spill $\times$ Discharge & 18 & $7,478.79$ & 30.25 & 34.10 & $7,442.694$ \\
\hline Photoperiod + Weight $+\%$ Spill + Discharge + Temperature $+\%$ Spill $\times$ Discharge & 19 & $7,448.54$ & 0.00 & 3.85 & $7,410.436$ \\
\hline
\end{tabular}


Table 20 (continued). Model selection for fish that passed through the spillway at McNary Dam relating survival ( $\varnothing)$ as a function of environmental variables and group covariates for spillway passage location (i.e. north, south) using the fixed structure of the best model for $p$ and $\lambda$ parameters for juvenile steelhead, 2004-09.

[The best-fit model is indicated in bold]

\begin{tabular}{|c|c|c|c|c|c|}
\hline Model & $\begin{array}{l}\text { Number of } \\
\text { parameters }\end{array}$ & $\mathrm{AIC} c$ & $\begin{array}{l}\Delta \mathrm{AIC} c \\
\text { (group) }\end{array}$ & $\begin{array}{c}\Delta \text { AIC } c \\
\text { (overall) }\end{array}$ & Deviance \\
\hline SpillPassLoc & 15 & $7,487.75$ & 43.06 & 43.06 & 171.802 \\
\hline SpillPassLoc + Temperature & 16 & $7,477.12$ & 32.44 & 32.44 & $7,445.048$ \\
\hline SpillPassLoc $+\%$ Spill & 16 & $7,484.92$ & 40.23 & 40.23 & $7,452.844$ \\
\hline SpillPassLoc + Discharge & 16 & $7,489.65$ & 44.96 & 44.96 & $7,457.571$ \\
\hline SpillPassLoc + Weight & 16 & $7,475.34$ & 30.65 & 30.65 & $7,443.265$ \\
\hline SpillPassLoc + Temperature $+\%$ Spill & 17 & $7,464.85$ & 20.17 & 20.17 & $7,430.768$ \\
\hline SpillPassLoc + Temperature + Discharge & 17 & $7,472.11$ & 27.42 & 27.42 & $7,438.026$ \\
\hline SpillPassLoc + Temperature + Weight & 17 & $7,465.46$ & 20.77 & 20.77 & $7,431.372$ \\
\hline SpillPassLoc + Weight $+\%$ Spill & 17 & $7,472.39$ & 27.70 & 27.70 & $7,438.305$ \\
\hline SpillPassLoc + Weight + Discharge & 17 & $7,476.26$ & 31.57 & 31.57 & $7,442.172$ \\
\hline SpillPassLoc $+\%$ Spill + Discharge & 17 & $7,486.93$ & 42.24 & 42.24 & $7,452.840$ \\
\hline SpillPassLoc + Weight + Temperature + Discharge & 18 & $7,456.09$ & 11.40 & 11.40 & $7,419.993$ \\
\hline SpillPassLoc + Temperature + Discharge $+\%$ Spill & 18 & $7,460.00$ & 15.31 & 15.31 & $7,423.906$ \\
\hline SpillPassLoc + Weight $+\%$ Spill + Discharge & 18 & $7,473.97$ & 29.28 & 29.28 & $7,437.875$ \\
\hline SpillPassLoc $+\%$ Spill + Discharge $+\%$ Spill $\times$ Discharge & 18 & $7,485.13$ & 40.44 & 40.44 & $7,449.033$ \\
\hline SpillPassLoc + Weight + Temperature + Discharge $+\%$ Spill & 19 & $7,445.72$ & 1.03 & 1.03 & 7,407.609 \\
\hline SpillPassLoc + Temperature + Discharge $+\%$ Spill $+\%$ Spill $\times$ Discharge & 19 & $7,459.03$ & 14.34 & 14.34 & $7,420.921$ \\
\hline SpillPassLoc + Weight $+\%$ Spill + Discharge $+\%$ Spill $\times$ Discharge & 19 & $7,471.97$ & 27.29 & 27.29 & $7,433.867$ \\
\hline SpillPassLoc + Weight + Temperature + Discharge $+\%$ Spill $+\%$ Spill $\times$ Discharge & 20 & $7,444.69$ & 0.00 & 0.00 & $7,404.570$ \\
\hline
\end{tabular}


Table 20 (continued). Model selection for fish that passed through the spillway at McNary Dam relating survival ( $\varnothing)$ as a function of environmental variables and group covariates for spillway passage location (i.e. north, south) and photoperiod using the fixed structure of the best model for $p$ and $\lambda$ parameters for juvenile steelhead, 2004-09.

\begin{tabular}{|c|c|c|c|c|c|}
\hline Model & $\begin{array}{l}\text { Number of } \\
\text { parameters }\end{array}$ & $\mathrm{AlC} c$ & $\begin{array}{l}\triangle \mathrm{AIC} c \\
\text { group }\end{array}$ & $\begin{array}{l}\Delta \mathrm{AIC} c \\
\text { overall }\end{array}$ & Deviance \\
\hline SpillPassLoc + Photoperiod & 16 & $7,488.14$ & 41.70 & 43.46 & 170.185 \\
\hline SpillPassLoc + Photoperiod $+\%$ Spill & 17 & $7,486.37$ & 39.93 & 41.69 & $7,452.289$ \\
\hline SpillPassLoc + Photoperiod + Temperature & 17 & $7,477.37$ & 30.93 & 32.69 & $7,443.289$ \\
\hline SpillPassLoc + Photoperiod + Discharge & 17 & $7,489.63$ & 43.19 & 44.94 & $7,455.543$ \\
\hline SpillPassLoc + Photoperiod + Weight $+\%$ Spill & 18 & $7,474.37$ & 27.93 & 29.68 & $7,438.273$ \\
\hline SpillPassLoc + Photoperiod + Weight + Temperature & 18 & $7,466.91$ & 20.47 & 22.22 & $7,430.812$ \\
\hline SpillPassLoc + Photoperiod + Weight + Discharge & 18 & $7,477.29$ & 30.85 & 32.61 & $7,441.199$ \\
\hline SpillPassLoc + Photoperiod $+\%$ Spill + Discharge & 18 & $7,488.29$ & 41.84 & 43.60 & $7,452.190$ \\
\hline SpillPassLoc + Photoperiod + Weight + Temperature + Discharge & 19 & $7,455.34$ & 8.90 & 10.65 & $7,417.232$ \\
\hline SpillPassLoc + Photoperiod $+\%$ Spill + Discharge + Temperature & 19 & $7,461.04$ & 14.60 & 16.35 & $7,422.936$ \\
\hline SpillPassLoc + Photoperiod + Weight $+\%$ Spill + Discharge & 19 & $7,475.83$ & 29.38 & 31.14 & $7,437.720$ \\
\hline SpillPassLoc + Photoperiod $+\%$ Spill + Discharge $+\%$ Spill $\times$ Discharge & 19 & $7,486.53$ & 40.09 & 41.85 & $7,448.427$ \\
\hline SpillPassLoc + Photoperiod + Weight $+\%$ Spill + Discharge + Temperature & 20 & $7,447.43$ & 0.98 & 2.74 & $7,407.308$ \\
\hline SpillPassLoc + Photoperiod $+\%$ Spill + Discharge + Temperature $+\%$ Spill $\times$ Discharge & 20 & $7,460.16$ & 13.72 & 15.47 & $7,420.041$ \\
\hline SpillPassLoc + Photoperiod + Weight $+\%$ Spill + Discharge $+\%$ Spill $\times$ Discharge & 20 & $7,473.85$ & 27.41 & 29.17 & $7,433.735$ \\
\hline SpillPassLoc + Photoperiod + Weight $+\%$ Spill + Discharge + Temperature $+\%$ Spill $\times$ Discharge & 21 & $7,446.44$ & 0.00 & 1.75 & $7,404.313$ \\
\hline
\end{tabular}



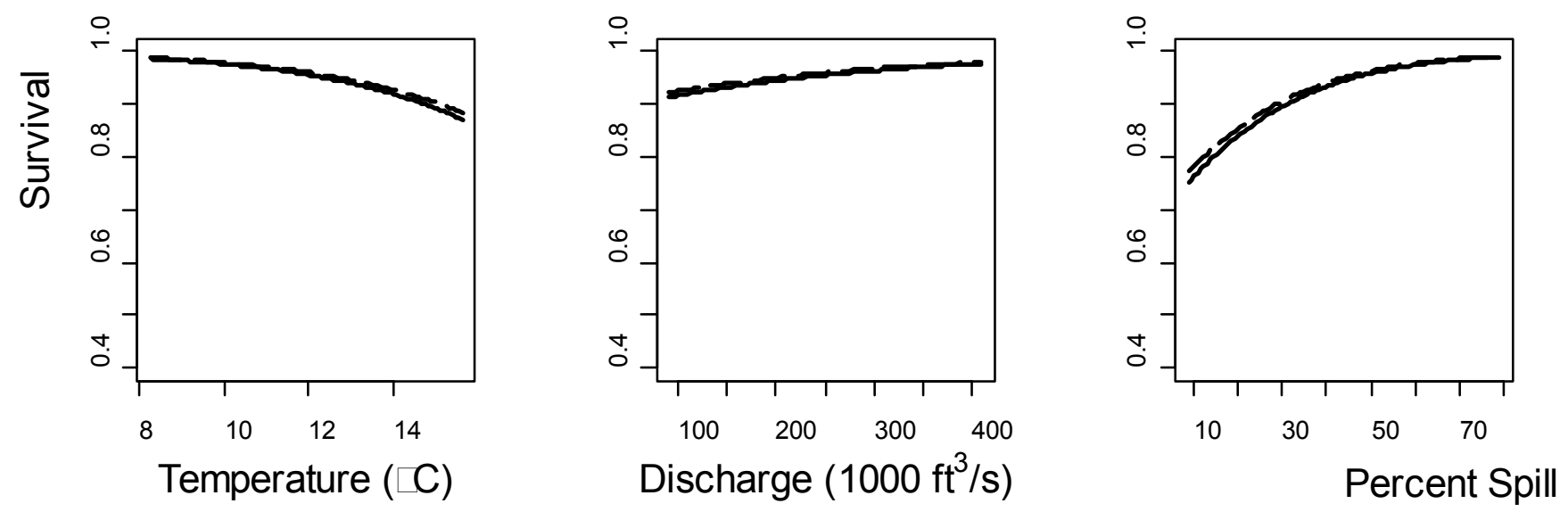

Figure 23. Survival of juvenile steelhead that passed through the north (solid line) and south (dashed line) portion of the spillway at McNary Dam in relation to temperature, total discharge, and percent spill, 2004-09. Other covariates in the model were held constant at their mean value to examine the relationship to the variable of interest. 

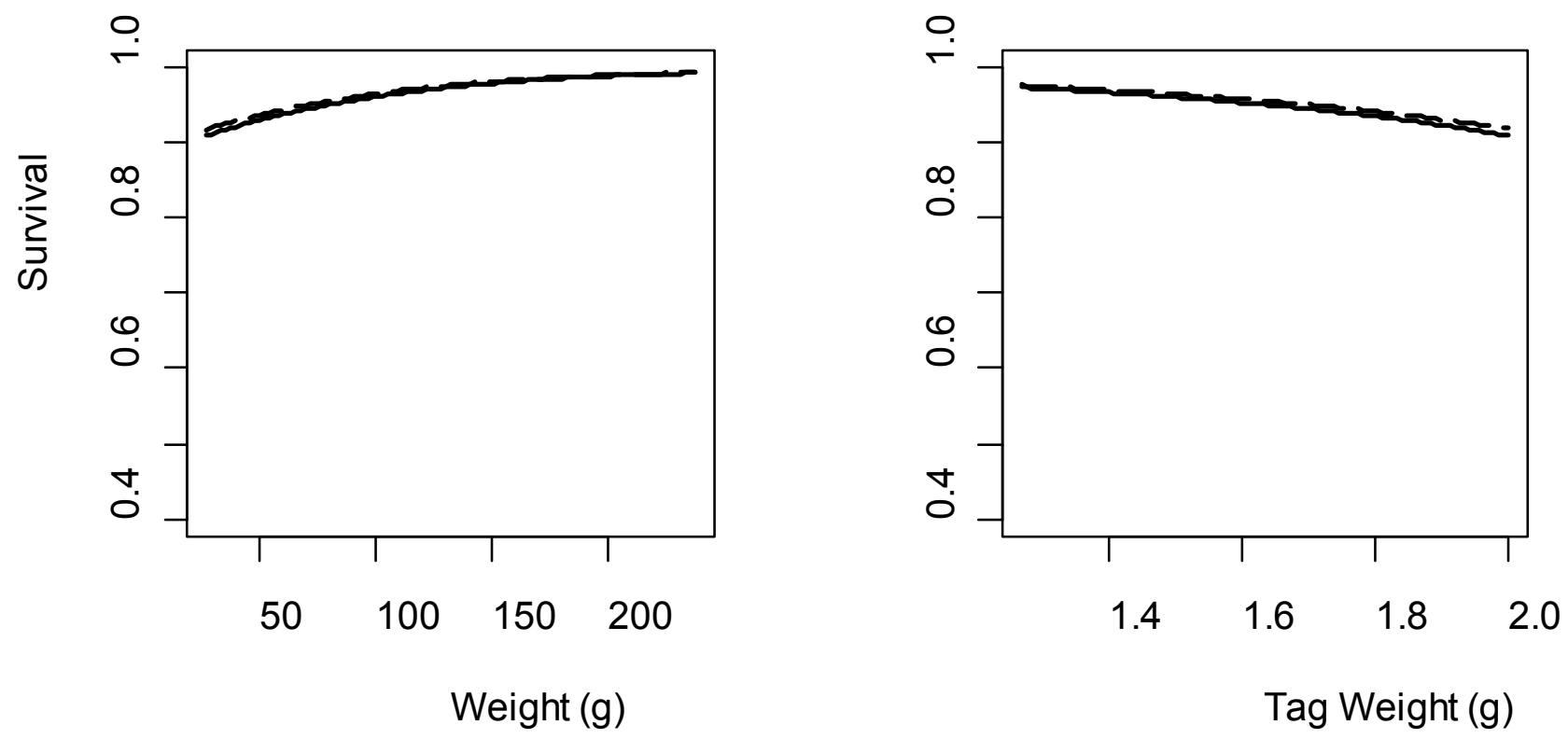

Figure 24. Survival of juvenile steelhead that passed through the north (solid line) and south (dashed line) portion of the spillway at McNary Dam in relation to fish weight and tag weight, 2004-09. Other covariates in the model were held constant at their mean value to examine the relationship to the variable of interest. 
Table 21. Model selection for fish released in the tailrace of McNary Dam relating survival ( $\varnothing)$ as a function of environmental variables using the fixed structure of the best model for $p$ and $\lambda$ parameters for juvenile steelhead, 2004-09.

\begin{tabular}{|c|c|c|c|c|c|}
\hline Model & $\begin{array}{l}\text { Number of } \\
\text { parameters }\end{array}$ & $\mathrm{AIC} c$ & $\begin{array}{l}\Delta \mathrm{AlC} c \\
\text { (group) }\end{array}$ & $\begin{array}{c}\Delta \mathrm{AIC} c \\
\text { (overall) }\end{array}$ & Deviance \\
\hline Intercept & 5 & $3,790.39$ & 6.42 & 7.31 & 29.538 \\
\hline$\%$ Spill & 6 & $3,788.72$ & 4.75 & 5.64 & $3,776.699$ \\
\hline Weight & 6 & $3,790.60$ & 6.62 & 7.52 & $3,778.575$ \\
\hline Discharge & 6 & $3,791.55$ & 7.58 & 8.47 & $3,779.531$ \\
\hline Temperature & 6 & $3,792.34$ & 8.36 & 9.26 & $3,780.315$ \\
\hline \%Spill + Discharge & 7 & $3,785.63$ & 1.66 & 2.56 & $3,771.606$ \\
\hline Temperature $+\%$ Spill & 7 & $3,790.67$ & 6.70 & 7.59 & $3,776.642$ \\
\hline Temperature + Discharge & 7 & $3,793.33$ & 9.36 & 10.25 & $3,779.301$ \\
\hline Weight $+\%$ Spill + Discharge & 8 & $3,785.41$ & 1.44 & 2.33 & $3,769.376$ \\
\hline Temperature + Discharge $+\%$ Spill & 8 & $3,786.42$ & 2.45 & 3.34 & $3,770.388$ \\
\hline Weight + Temperature $+\%$ Spill & 8 & $3,791.11$ & 7.14 & 8.03 & $3,775.072$ \\
\hline Weight + Temperature + Discharge & 8 & $3,793.04$ & 9.07 & 9.96 & $3,777.004$ \\
\hline Weight $+\%$ Spill + Discharge $+\%$ Spill $\times$ Discharge & 9 & $3,783.97$ & 0.00 & 0.89 & $3,765.927$ \\
\hline Temperature + Discharge $+\%$ Spill $+\%$ Spill $\times$ Discharge & 9 & $3,786.56$ & 2.59 & 3.48 & $3,768.514$ \\
\hline Weight + Temperature + Discharge $+\%$ Spill & 9 & $3,785.72$ & 1.75 & 2.64 & $3,767.673$ \\
\hline Temperature + Discharge $+\%$ Spill $+\%$ Spill $\times$ Discharge & 9 & $3,786.56$ & 2.59 & 3.48 & $3,768.514$ \\
\hline Weight + Temperature + Discharge $+\%$ Spill $+\%$ Spill $\times$ Discharge & 10 & $3,785.79$ & 1.82 & 2.71 & $3,765.732$ \\
\hline
\end{tabular}


Table 21 (continued). Model selection for fish released in the tailrace of McNary Dam relating survival $(\varnothing)$ as a function of environmental variables and group covariates for photoperiod using the fixed structure of the best model for $p$ and $\lambda$ parameters for juvenile steelhead, 2004-09.

[The best-fit model is indicated in bold]

\begin{tabular}{|c|c|c|c|c|c|}
\hline Model & $\begin{array}{l}\text { Number of } \\
\text { parameters }\end{array}$ & $\mathrm{AlC} c$ & $\begin{array}{c}\Delta \mathrm{AlC} c \\
\text { (group) }\end{array}$ & $\begin{array}{c}\Delta \mathrm{AIC} c \\
\text { (overall) }\end{array}$ & Deviance \\
\hline Photoperiod & 6 & $3,785.49$ & 2.42 & 2.42 & 22.635 \\
\hline Photoperiod $+\%$ Spill & 7 & $3,785.87$ & 2.79 & 2.79 & $3,771.838$ \\
\hline Photoperiod + Discharge & 7 & $3,786.85$ & 3.77 & 3.77 & $3,772.818$ \\
\hline Photoperiod + Weight & 7 & $3,785.28$ & 2.20 & 2.20 & $3,771.251$ \\
\hline Photoperiod + Temperature & 7 & $3,787.28$ & 4.20 & 4.20 & $3,773.248$ \\
\hline Photoperiod + \%Spill + Discharge & 8 & $3,784.67$ & 1.59 & 1.59 & $3,768.635$ \\
\hline Photoperiod + Weight $+\%$ Spill & 8 & $3,785.84$ & 2.76 & 2.76 & $3,769.805$ \\
\hline Photoperiod + Weight + Discharge & 8 & $3,786.40$ & 3.33 & 3.33 & $3,770.369$ \\
\hline Photoperiod + Weight + Temperature & 8 & $3,786.95$ & 3.87 & 3.87 & $3,770.909$ \\
\hline Photoperiod $+\%$ Spill + Discharge $+\%$ Spill $\times$ Discharge & 9 & $3,783.97$ & 0.89 & 0.89 & $3,765.927$ \\
\hline Photoperiod + Weight $+\%$ Spill + Discharge & 9 & $3,784.21$ & 1.13 & 1.13 & $3,766.161$ \\
\hline Photoperiod $+\%$ Spill + Discharge + Temperature & 9 & $3,785.44$ & 2.36 & 2.36 & $3,767.397$ \\
\hline Photoperiod + Weight $+\%$ Spill + Discharge $+\%$ Spill $\times$ Discharge & 10 & $3,783.08$ & 0.00 & 0.00 & $3,763.024$ \\
\hline Photoperiod + Weight $+\%$ Spill + Discharge + Temperature & 10 & $3,784.47$ & 1.39 & 1.39 & $3,764.413$ \\
\hline Photoperiod $+\%$ Spill + Discharge + Temperature $+\%$ Spill $\times$ Discharge & 10 & $3,785.88$ & 2.80 & 2.80 & $3,765.824$ \\
\hline Photoperiod + Weight $+\%$ Spill + Discharge + Temperature $+\%$ Spill $\times$ Discharge & 11 & $3,784.85$ & 1.77 & 1.77 & $3,762.782$ \\
\hline
\end{tabular}



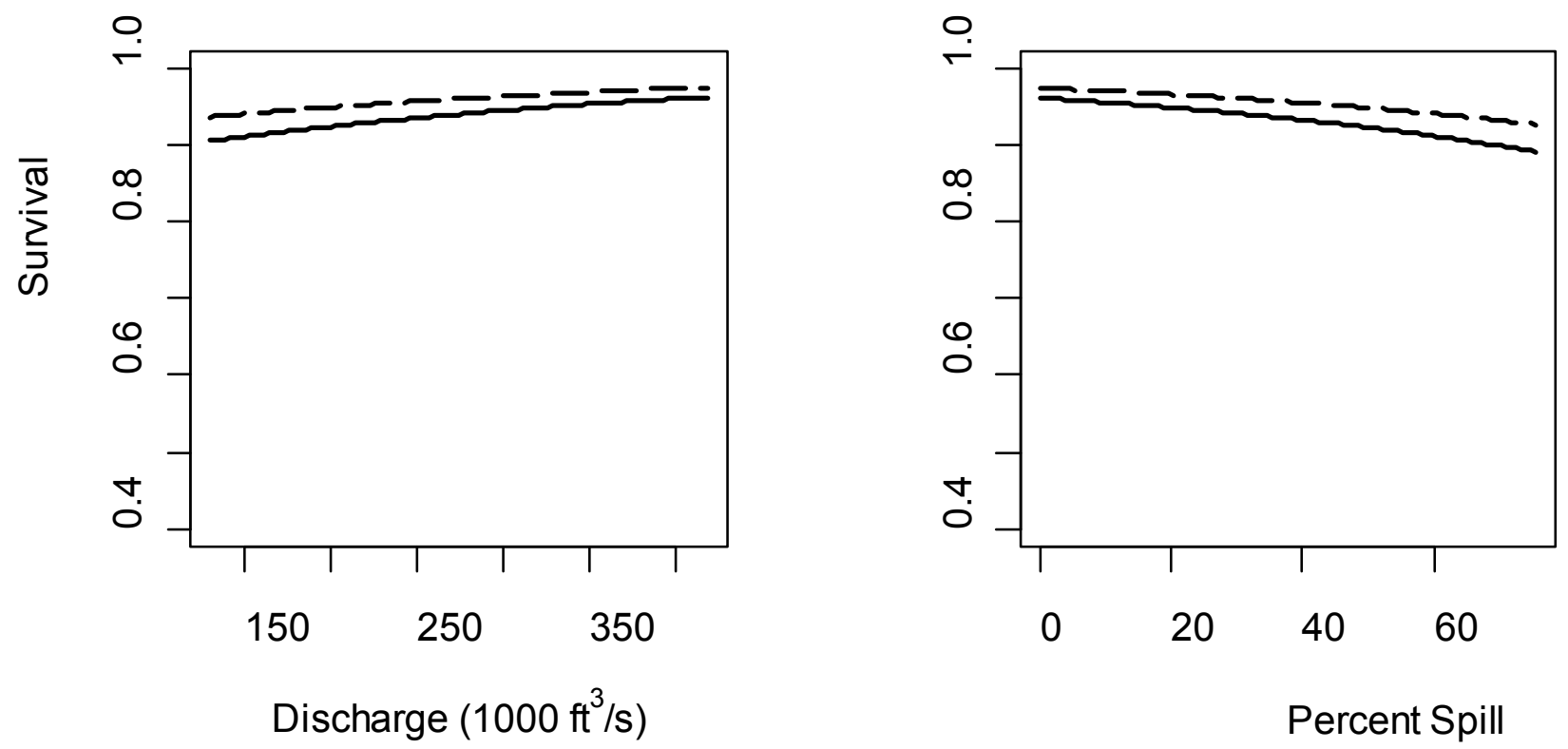

Figure 25. Survival of juvenile steelhead that were released in the tailrace of McNary Dam related to total discharge and percent spill during day (dashed line) and night (solid line) photoperiods, 2004-09. Other covariates in the model were held constant at their mean value to examine the relationship to the variable of interest. 

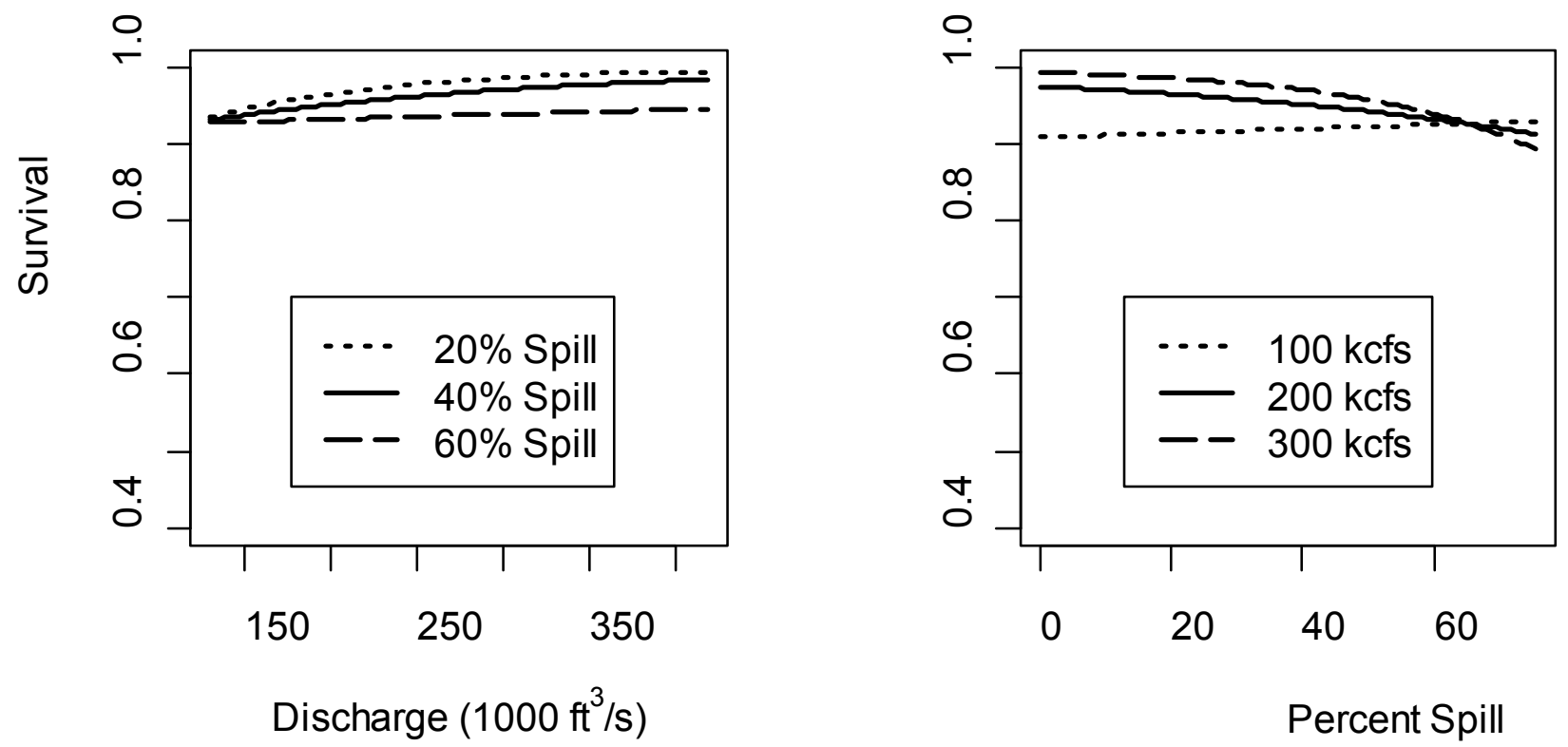

Figure 26. Survival of juvenile steelhead released in the tailrace of McNary Dam in relation to discharge and percent spill at varying levels during the day photoperiod, 2004-09. Other covariates in the model were held constant at their mean value to examine the relationship to the variable of interest. 

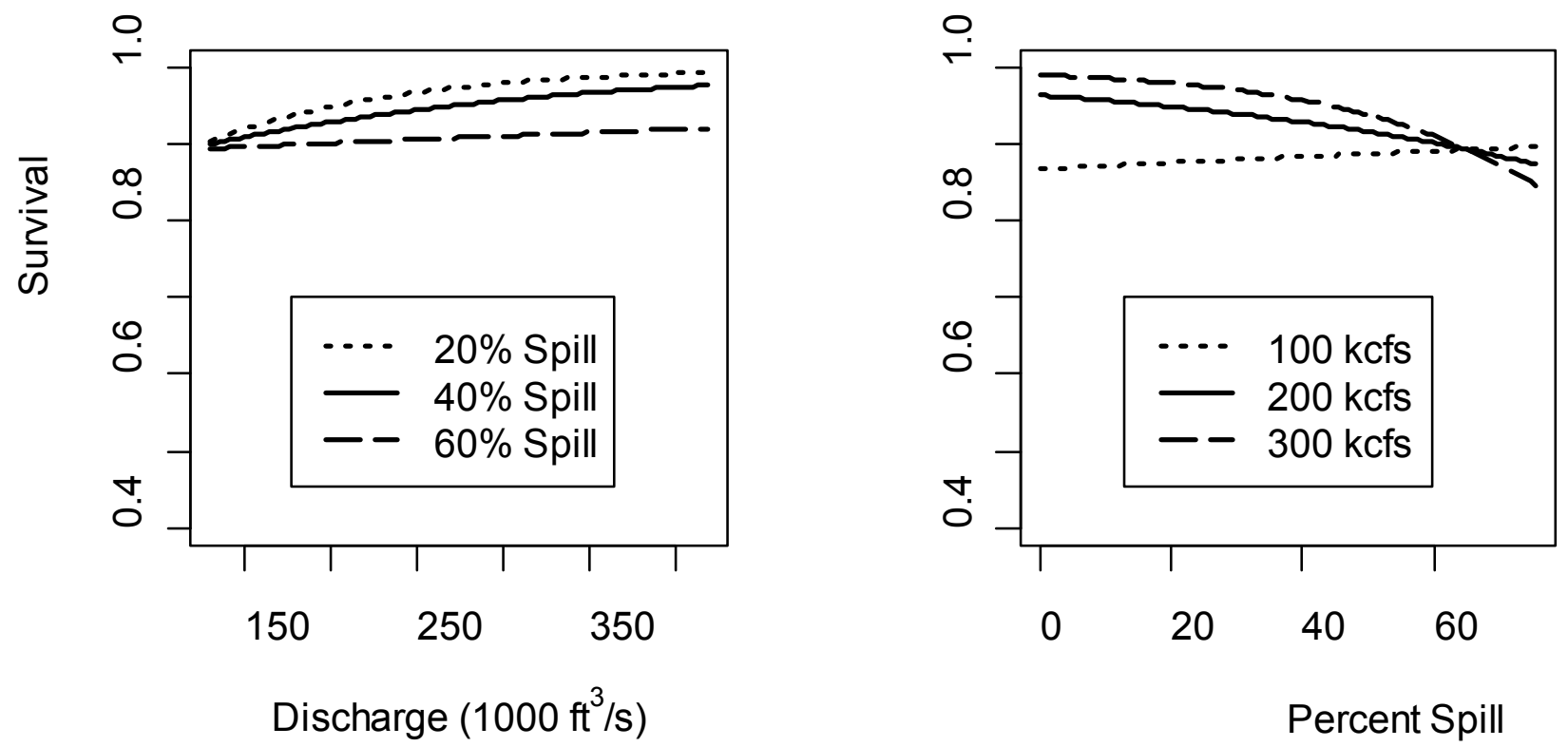

Figure 27. Survival of juvenile steelhead released in the tailrace of McNary Dam in relation to discharge and percent spill at varying levels during the night photoperiod, 2004-09. Other covariates in the model were held constant at their mean value to examine the relationship to the variable of interest. 


\section{Temporal Survival Estimates}

Survival estimates at the weekly (table A2) and annual (table 22) temporal scale were generated to provide temporal information for survival through routes and to compare estimates predicted by covariates. Weekly survival was fairly consistent for all years, especially as fish traveled through the forebay or for those that passed the spillway, juvenile bypass system, or were released in the tailrace of McNary Dam (figs. 28 and 29). Survival in the forebay decreased in late spring during 2005, as was observed with yearling Chinook salmon. Weekly survival for fish that passed through turbines was much more variable. Weekly survival for juvenile steelhead that went through turbines ranged from 0.250 to 1.00 . Furthermore, weekly survival for fish that passed the juvenile bypass system ranged from 0.882 to 1.00. In contrast, survival for fish released in the tailrace of McNary Dam ranged from 0.902 to 1.00 and those that passed through the spillway ranged from 0.823 to 1.00 . Covariates predicted weekly survival estimates well for fish passing through the spillway, juvenile bypass system, and for fish released in the tailrace of McNary Dam (figs. 30 and 31). At the annual temporal scale, covariates predicted survival better than at the weekly temporal scale (table 22, fig. 32).

Table 22. Annual survival estimates during the spring migration period for juvenile steelhead passing through, and released in the tailrace of, McNary Dam, 2004-09.

[Standard error is in parentheses]

\begin{tabular}{lcccccc}
\hline & $\mathbf{2 0 0 4}$ & $\mathbf{2 0 0 5}$ & $\mathbf{2 0 0 6}$ & $\mathbf{2 0 0 7}$ & $\mathbf{2 0 0 8}$ & $\mathbf{2 0 0 9}$ \\
\hline Forebay & NA & $0.939(0.009)$ & $0.995(0.003)$ & $0.995(0.003)$ & $0.996(0.002)$ & $0.994(0.003)$ \\
Turbine & $0.787(0.060)$ & $0.744(0.059)$ & $0.880(0.048)$ & $0.702(0.090)$ & $0.744(0.059)$ & $0.782(0.056)$ \\
Bypass & $0.923(0.022)$ & $0.911(0.018)$ & $0.975(0.018)$ & $0.896(0.041)$ & $0.999(0.015)$ & $0.960(0.012)$ \\
Spillway & $0.939(0.010)$ & $0.938(0.012)$ & $0.986(0.011)$ & $0.914(0.014)$ & $0.978(0.008)$ & $0.952(0.008)$ \\
Tailrace/Control & $0.936(0.011)$ & NA & NA & NA & $0.967(0.009)$ & $0.951(0.007)$ \\
\hline
\end{tabular}




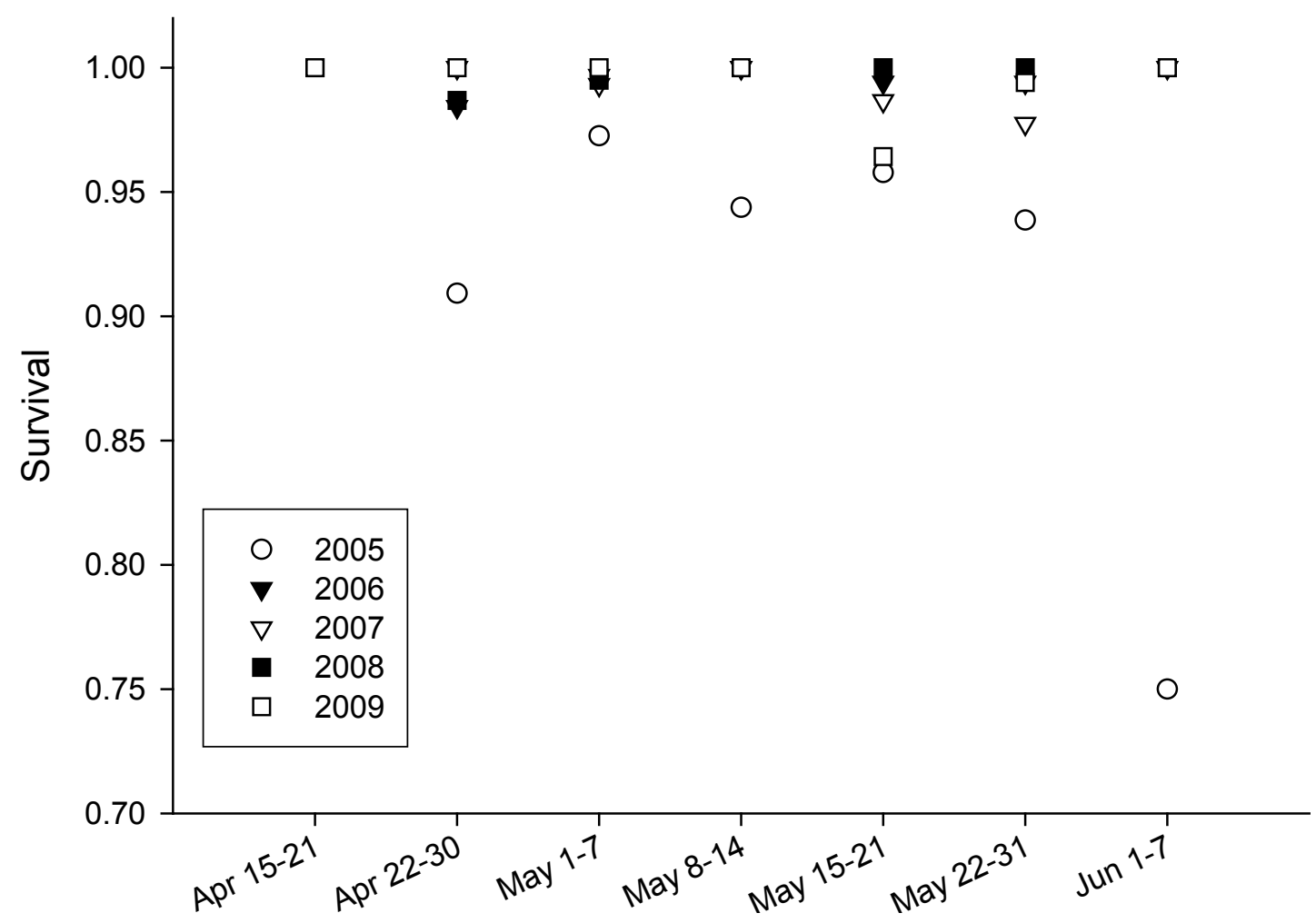

Figure 28. Weekly survival of juvenile steelhead in the forebay of McNary Dam, 2005-09. 
Turbine

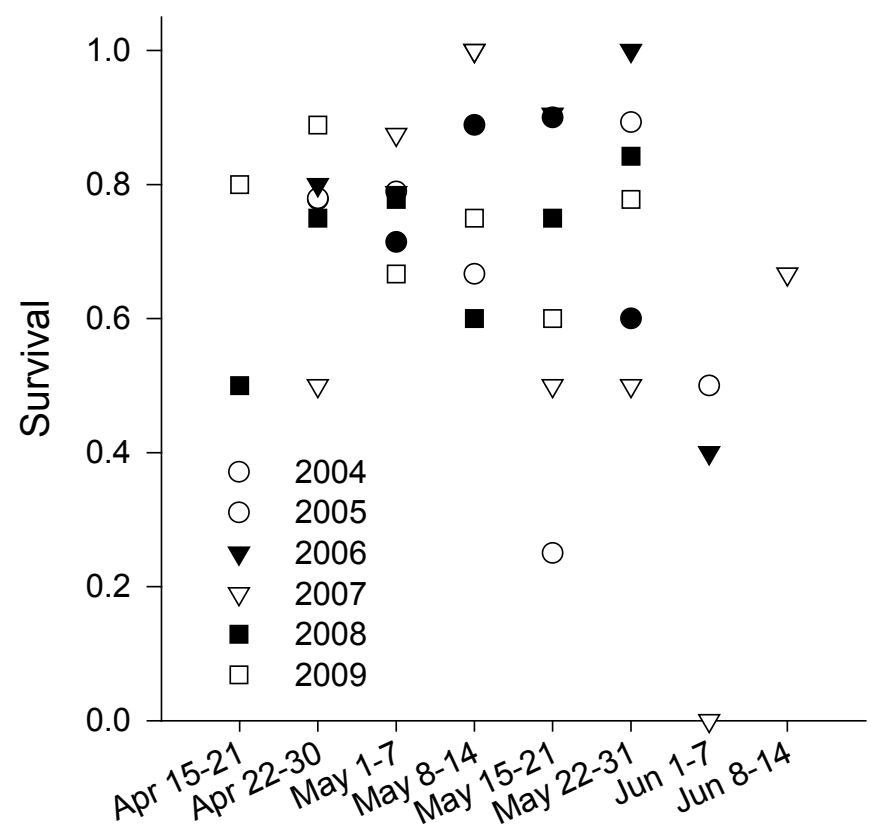

Spillway

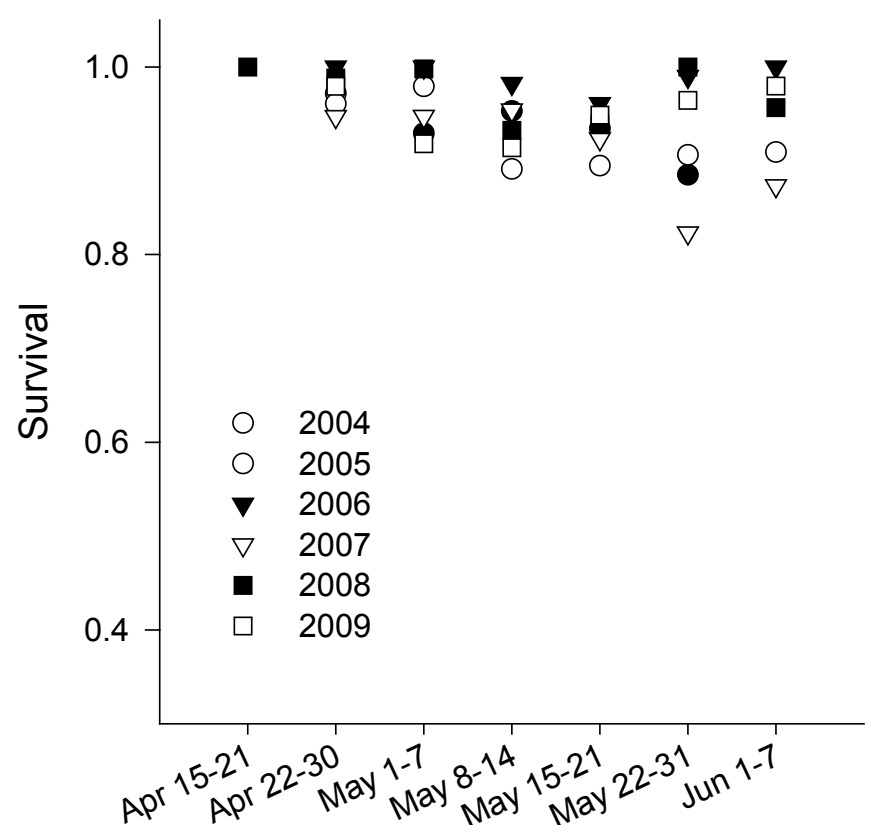

Bypass

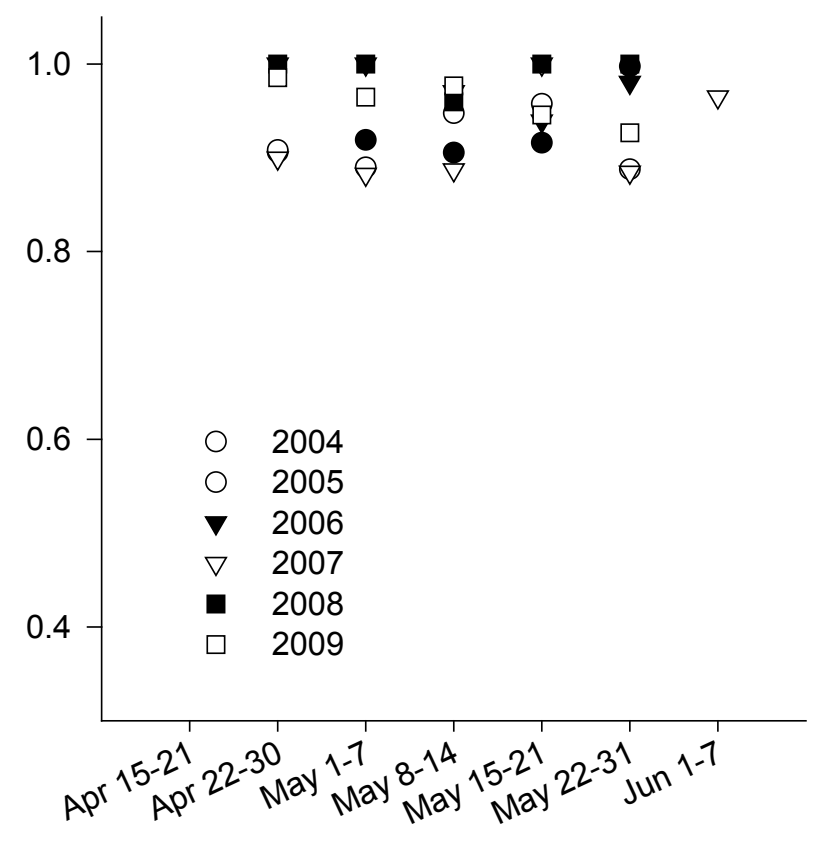

Tailrace/Control

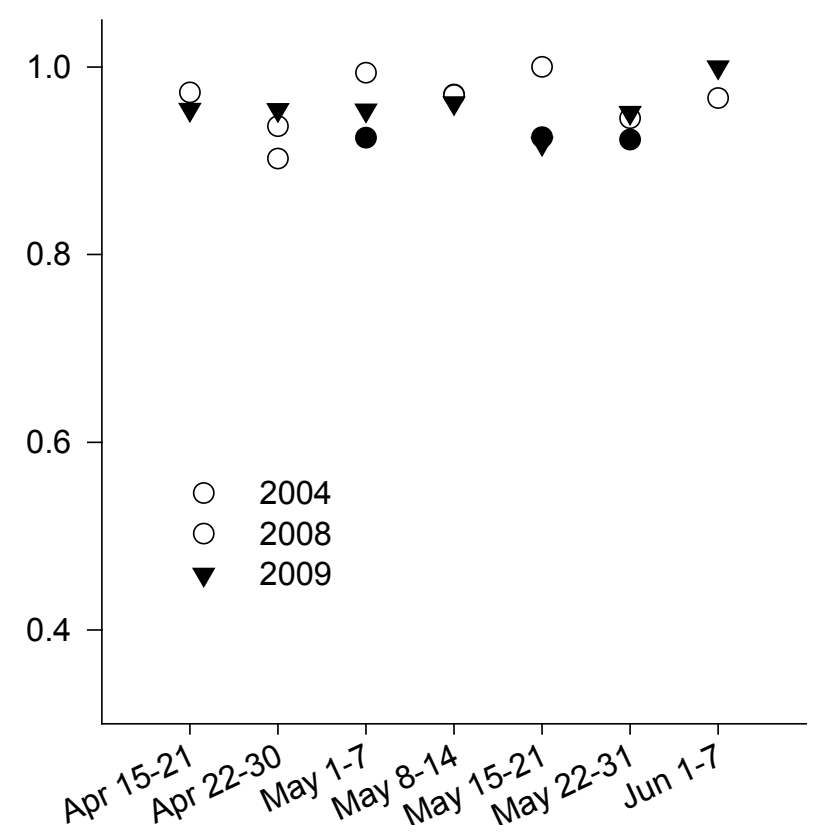

Figure 29. Weekly survival of juvenile steelhead that passed through the juvenile bypass system, spillway, turbines, or that were released in the tailrace of McNary Dam, 2004-09. 

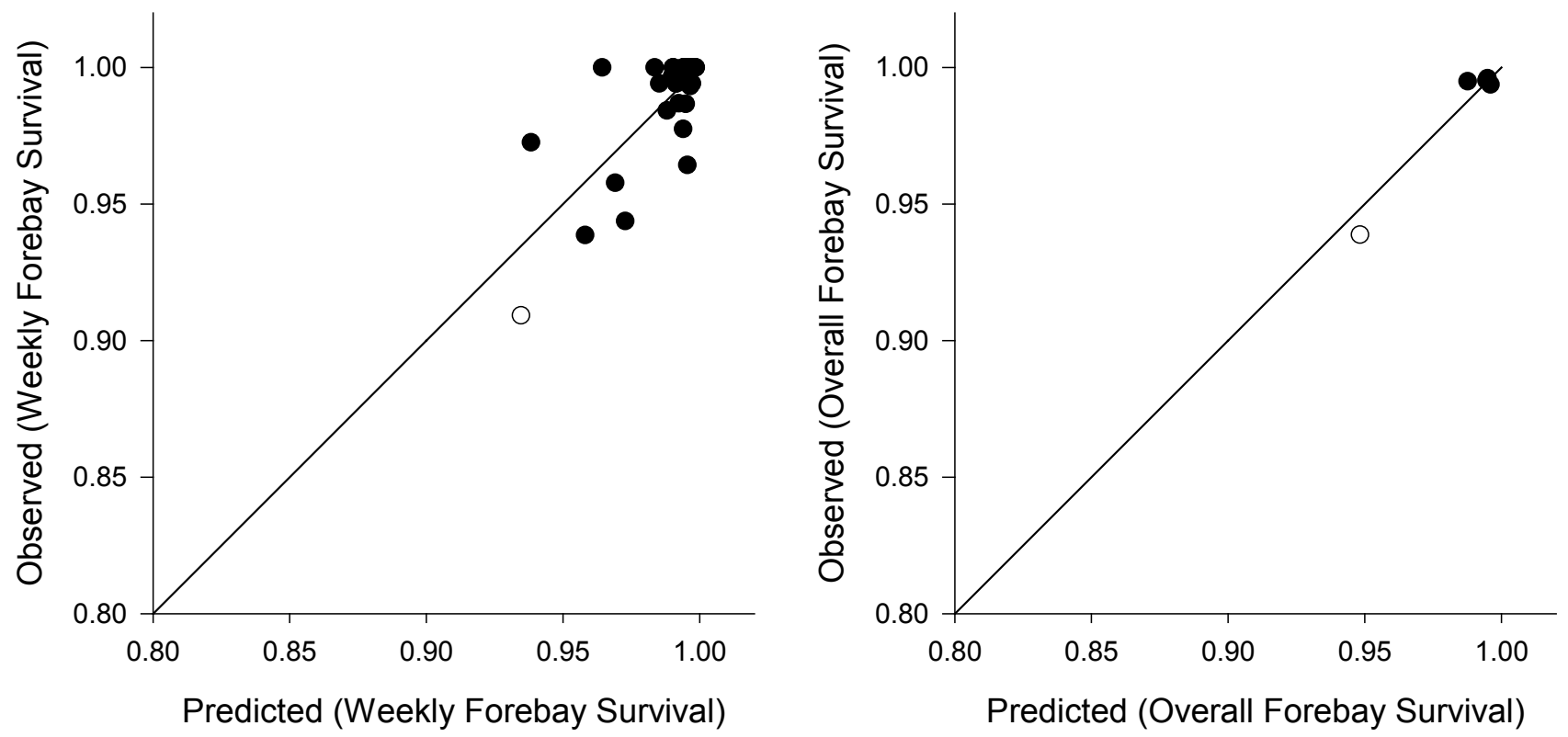

Figure 30. Observed versus predicted weekly (left) and annual survival (right) of juvenile steelhead during the spring migration period in the forebay of McNary Dam, 2005-09. Solid line indicates graph coordinates where observed and predicted survival are equivalent. 
Turbine

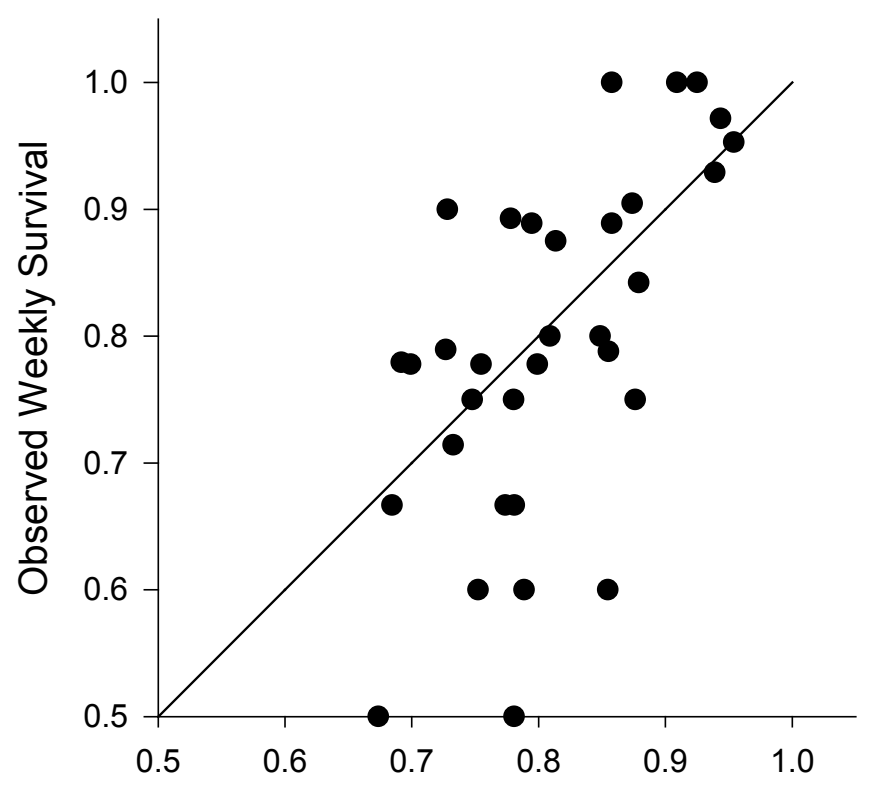

Spillway

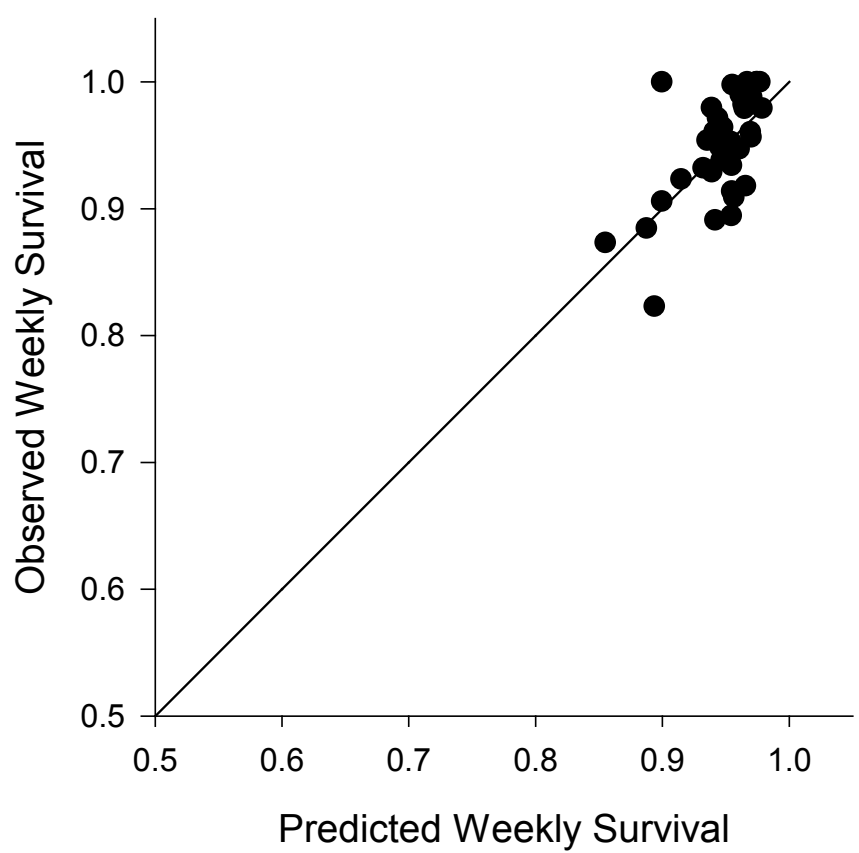

Bypass

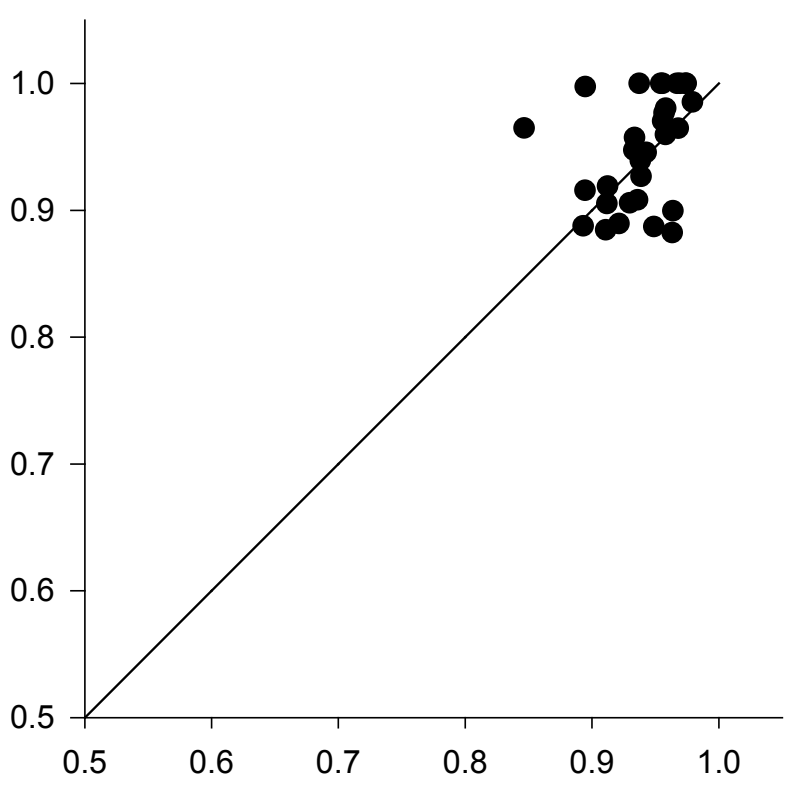

Tailrace/Control

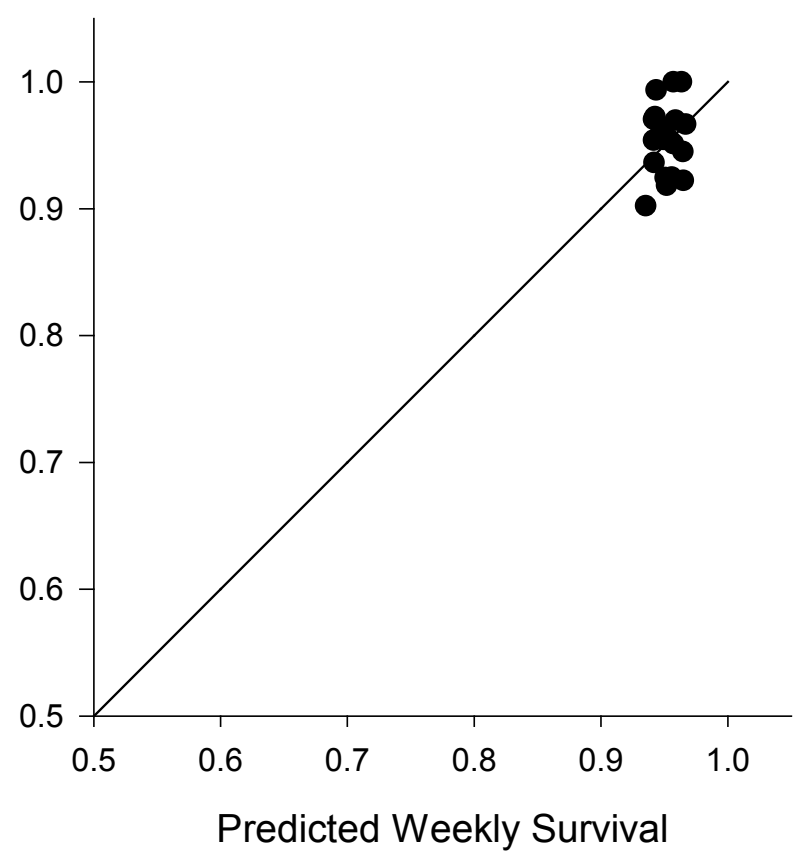

Figure 31. Observed versus predicted weekly survival of juvenile steelhead that passed through the juvenile bypass system, spillway, turbines, or that were released in the tailrace of McNary Dam, 2004-09. Solid line indicates graph coordinates where observed and predicted survival are equivalent. 
Turbine

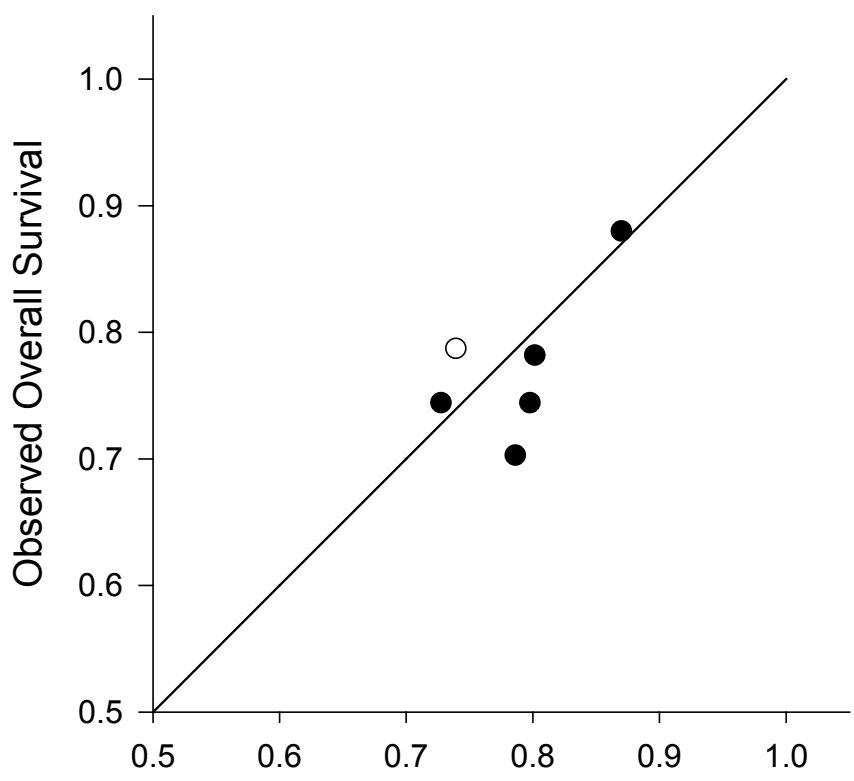

Spillway

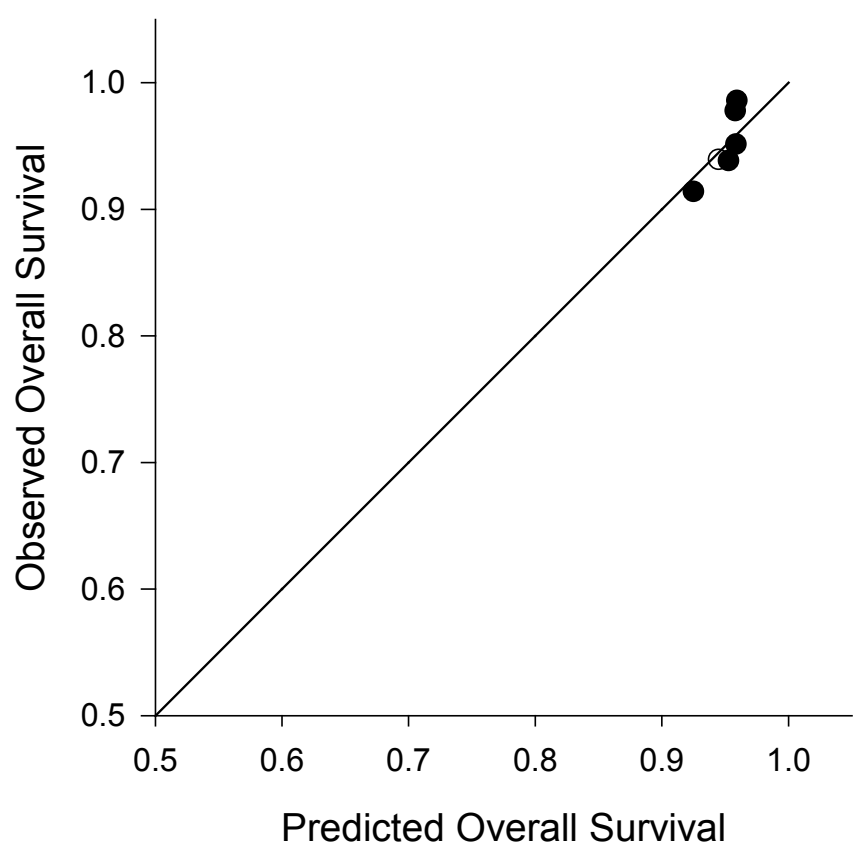

Bypass

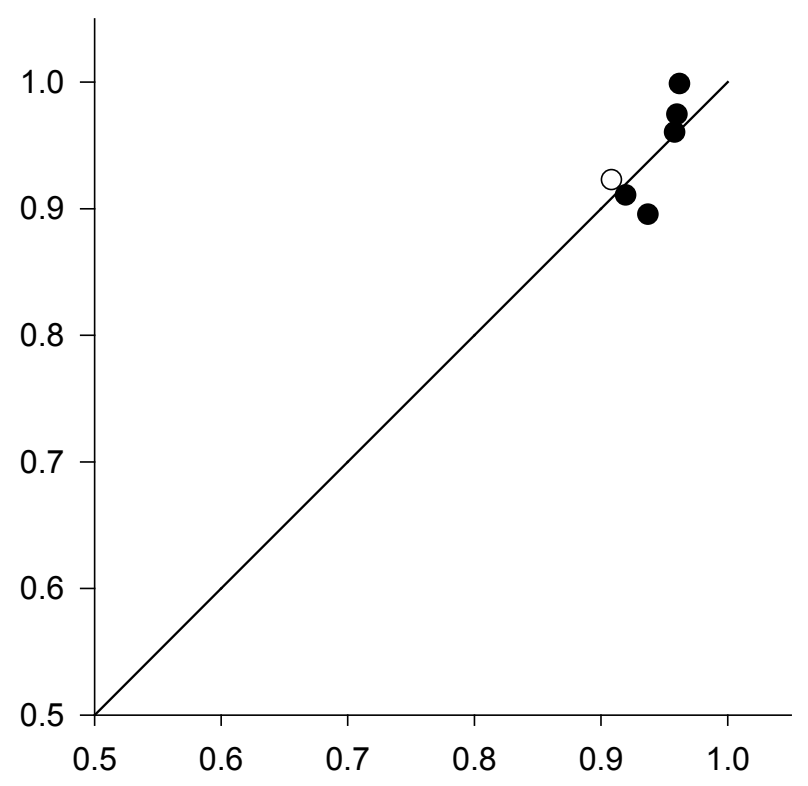

Tailrace/Control

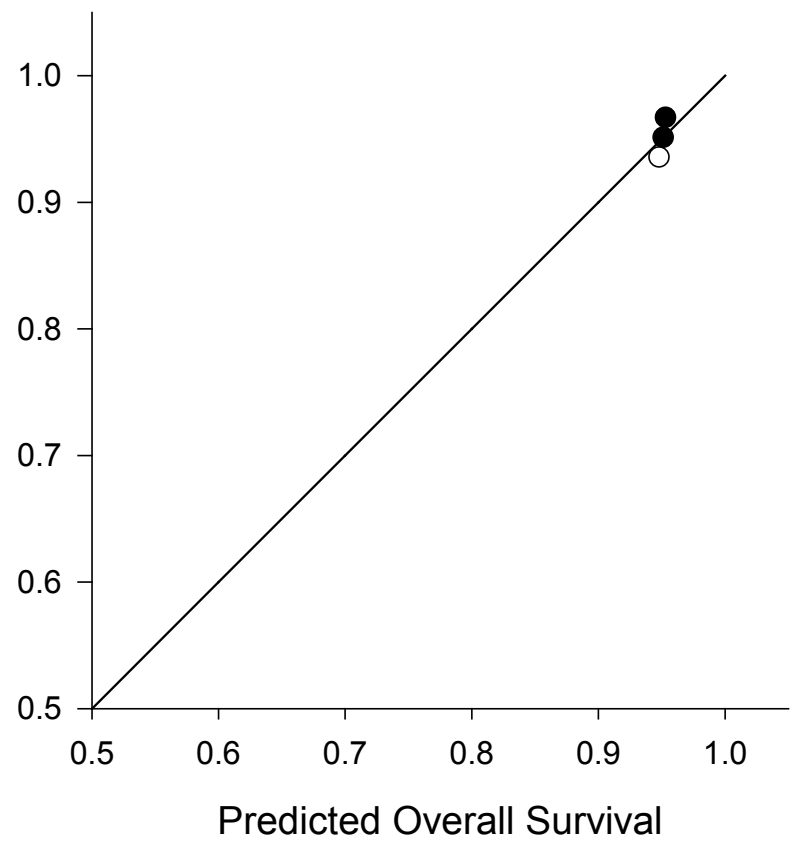

Figure 32. Observed versus predicted annual survival of juvenile steelhead during the spring migration period for fish that passed through the juvenile bypass system, spillway, turbines, or that were released in the tailrace of McNary Dam, 2004-09. Solid line indicates graph coordinates where observed and predicted survival are equivalent. 


\section{Subyearling Chinook Salmon}

\section{Passage Analysis}

The best model explaining passage probabilities at McNary Dam included covariates for photoperiod, percent spill, fish weight, and water temperature for all routes (table 23). As percent spill increased, more fish passed the dam through the spillway, and less passed through both turbines and the juvenile bypass system (table 24, figs. 33 and 36). Passage through all routes was relatively constant, compared to other species as discharge increased at the dam (fig. 36). As temperature increased, passage through the spillway and bypass decreased while it increased through turbines (fig. 34). As fish size increased, passage through the spillway also increased and decreased through turbines and the juvenile bypass system (fig. 35). A factor for whether a TSW was installed explained a significant amount of variation for passage and was positively $\left(\beta_{\mathrm{TSW}}=0.272\right)$ related to passage through the spillway only when a year factor was not included.

Table 23. Passage models for subyearling Chinook salmon that passed through McNary Dam, 2004-09, comparing the full model with models that have one variable removed.

(NLL, negative log likelihood; Df, degrees of freedom; $\chi^{2}$, chi-square statistic for likelihood ratio test]

\begin{tabular}{|c|c|c|c|c|c|c|}
\hline Model & $\begin{array}{l}\text { Number of } \\
\text { parameters }\end{array}$ & $\mathrm{AIC} c$ & NLL & $\chi^{2}$ & Df & $p$ \\
\hline \multicolumn{7}{|c|}{ Spillway } \\
\hline Full (all variables) & 14 & $15,850.72$ & $7,911.36$ & NA & NA & NA \\
\hline - Photoperiod & 13 & $16,085.22$ & $8,029.61$ & 236.51 & 1 & 0 \\
\hline - \%Spill & 13 & $16,079.70$ & $8,026.85$ & 230.98 & 1 & 0 \\
\hline - Discharge & 13 & $15,867.99$ & $7,920.99$ & 19.27 & 1 & 0 \\
\hline - Weight & 13 & $15,879.73$ & $7,926.87$ & 31.02 & 1 & 0 \\
\hline - Temperature & 13 & $15,955.45$ & $7,964.73$ & 106.74 & 1 & 0 \\
\hline - \%Spill $\times$ Discharge & 13 & $15,851.38$ & $7,912.69$ & 2.67 & 1 & 0.1023 \\
\hline \multicolumn{7}{|c|}{ Bypass } \\
\hline - Photoperiod & 13 & $15,871.88$ & $7,922.94$ & 23.16 & 1 & 0 \\
\hline - \%Spill & 13 & $15,912.25$ & $7,943.13$ & 63.54 & 1 & 0 \\
\hline - Discharge & 13 & $15,856.30$ & $7,915.15$ & 7.58 & 1 & 0.0059 \\
\hline - Weight & 13 & $15,852.98$ & $7,913.49$ & 4.27 & 1 & 0.0388 \\
\hline - Temperature & 13 & $15,936.20$ & $7,955.10$ & 87.48 & 1 & 0 \\
\hline$-\%$ Spill $\times$ Discharge & 13 & $15,855.28$ & 7,914.64 & 6.56 & 1 & 0.0104 \\
\hline
\end{tabular}



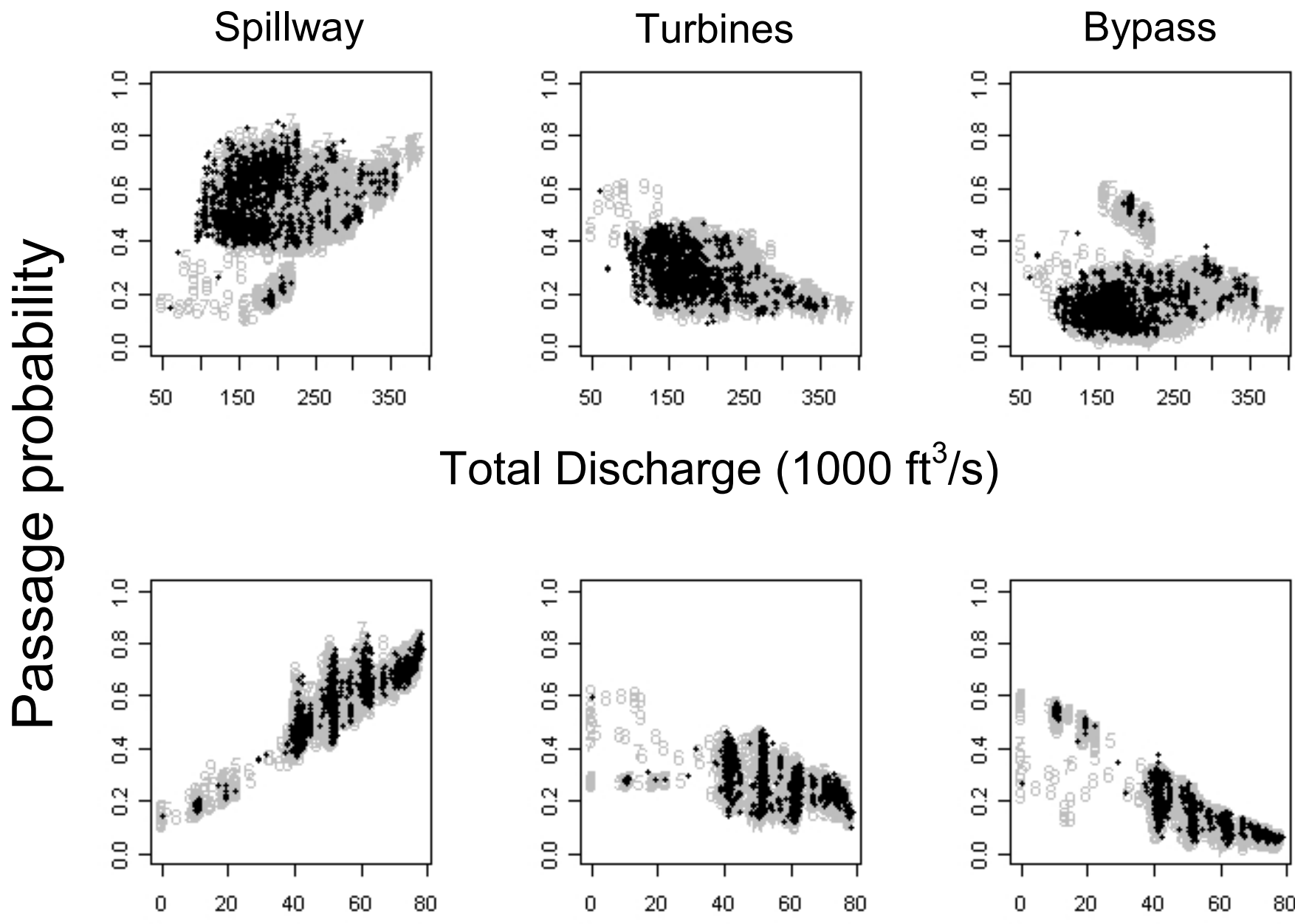

Total Discharge $\left(1000 \mathrm{ft}^{3} / \mathrm{s}\right)$
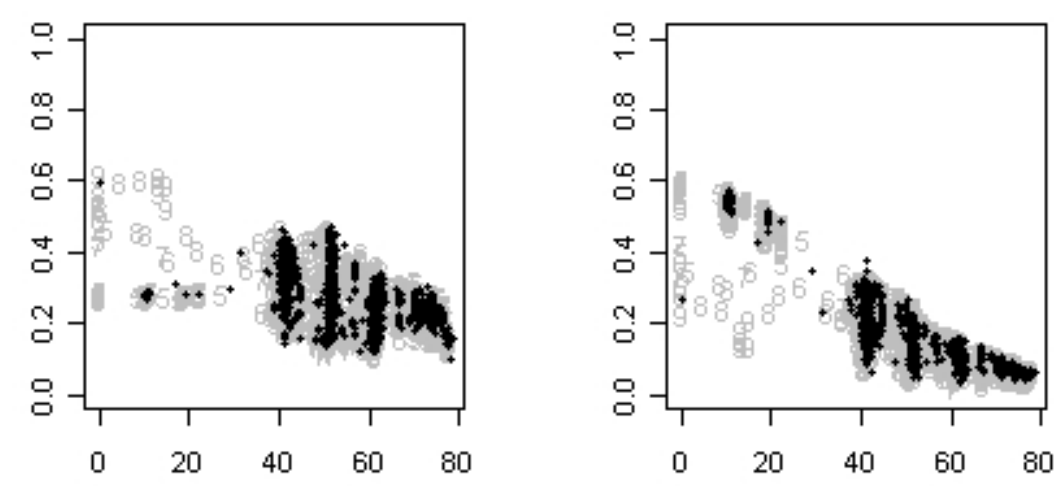

\section{Percent Spill}

Figure 33. Passage probabilities of subyearling Chinook salmon predicted by continuous covariates at McNary Dam through the spillway, turbines, and the juvenile bypass system in relation to total discharge and percent spill during day (grey symbols) and night (black symbols) photoperiods, 2004-09. Numbers used as plot symbols represent year of study, for example, $4=2004$ ). 

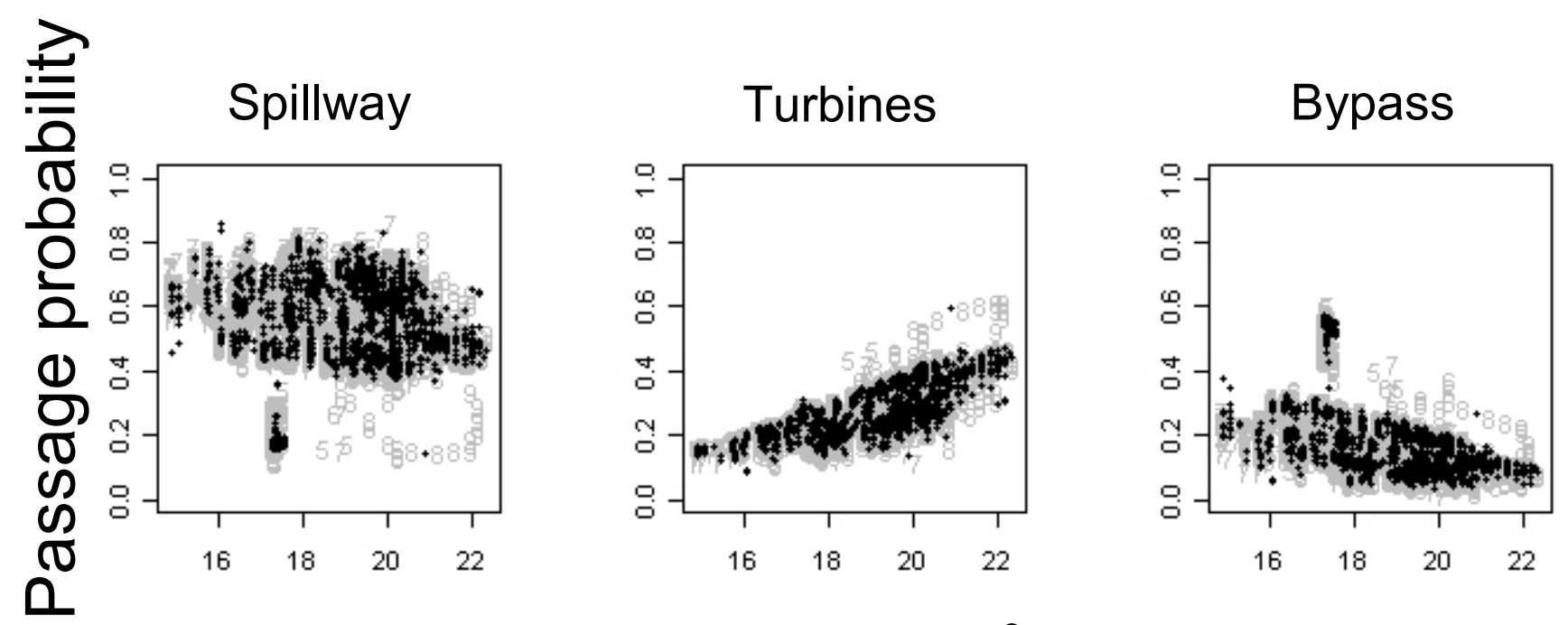

\section{Temperature ${ }^{\circ} \mathrm{C}$}

Figure 34. Passage probabilities of subyearling Chinook salmon predicted by continuous covariates at McNary Dam through the spillway, turbines, and the juvenile bypass system in relation to water temperature during day (grey symbols) and night (black symbols) photoperiods, 2004-09. Numbers used as plot symbols represent year of study, for example, 4=2004). 



Weight (g)

Figure 35. Passage probabilities of subyearling Chinook salmon predicted by continuous covariates at McNary Dam through the spillway, turbines, and juvenile bypass system in relation to fish weight, 2004-09. 


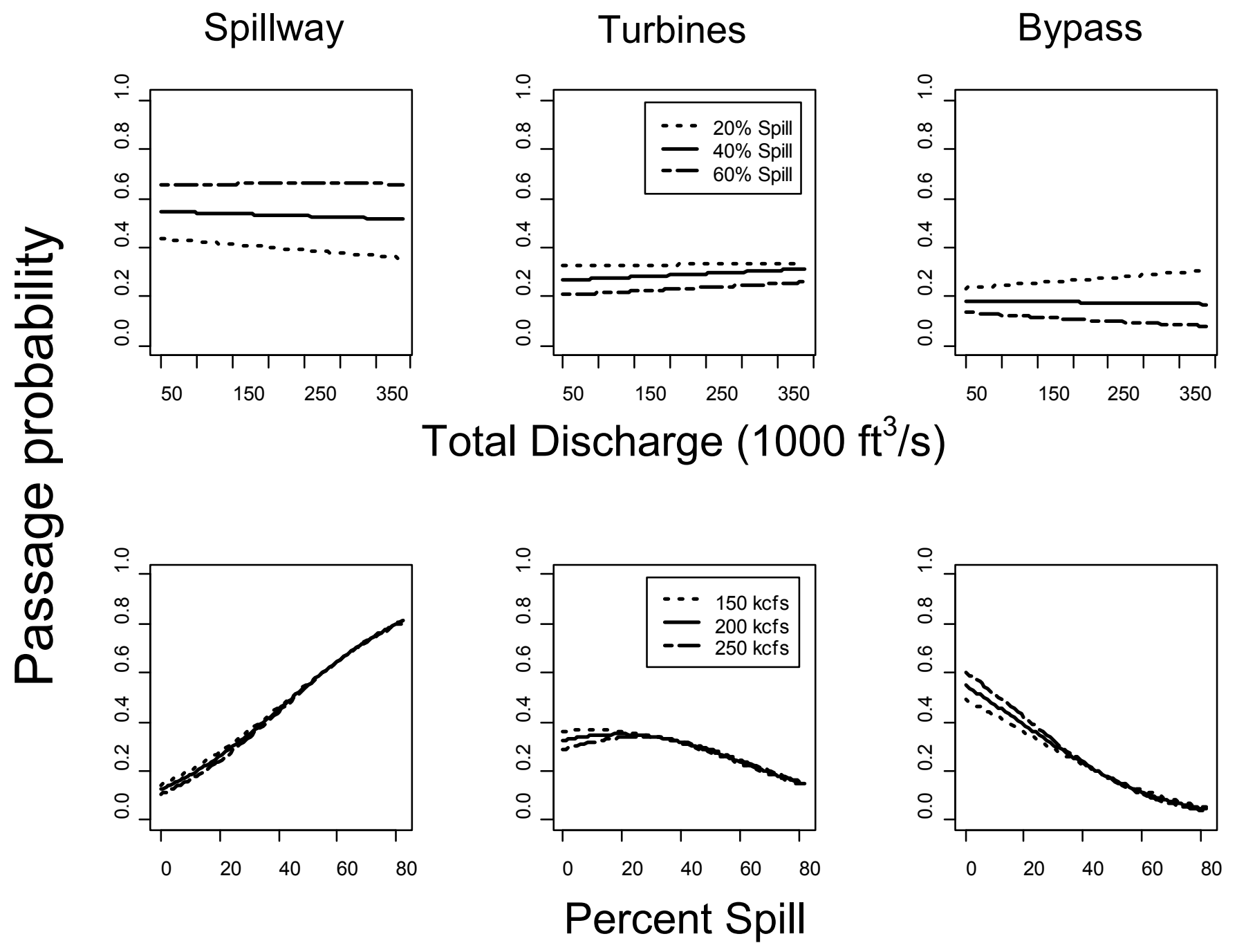

Figure 36. Passage probabilities of subyearling Chinook salmon related to total discharge and percent spill at McNary Dam through the spillway, turbines, and the juvenile bypass, 2004-09. Other covariates in the model were held constant at their mean value to examine the relationship to the variable of interest. 
Table 24. Slope (Beta) coefficients, standard error, and 95-percent confidence limits of model parameters of passage probabilities for subyearling Chinook salmon passing McNary Dam, 2004-09.

[CL, confidence limit]

\begin{tabular}{lcccc}
\hline \multicolumn{1}{c}{ Variable } & \multicolumn{1}{c}{ Beta } & $\begin{array}{c}\text { Standard } \\
\text { error }\end{array}$ & Lower CL & Upper CL \\
\hline \multicolumn{2}{c}{ Spillway } \\
Photoperiod (dark) & -0.025 & 0.056 & -0.134 & 0.085 \\
Photoperiod (light) & 0.987 & 0.064 & 0.861 & 1.113 \\
\%Spill & 0.468 & 0.029 & 0.412 & 0.525 \\
Discharge & -0.152 & 0.036 & -0.223 & -0.080 \\
Weight & 0.149 & 0.028 & 0.095 & 0.203 \\
Temperature & -0.371 & 0.036 & -0.442 & -0.299 \\
\hline & Bypass & & & \\
\hline Photoperiod (dark) & -0.862 & 0.072 & -1.004 & -0.720 \\
Photoperiod (light) & 0.394 & 0.083 & 0.231 & 0.557 \\
\%Spill & -0.275 & 0.036 & -0.345 & -0.205 \\
Discharge & -0.126 & 0.049 & -0.222 & -0.031 \\
Weight & -0.084 & 0.040 & -0.163 & -0.004 \\
Temperature & -0.452 & 0.049 & -0.547 & -0.356 \\
Discharge×\%Spill & -0.090 & 0.044 & -0.176 & -0.003 \\
\hline
\end{tabular}




\section{Survival Analysis}

For the majority of the analyses of subyearling Chinook salmon survival, the best model for $p$ and $\lambda$ was the model that expressed both of these with a year effect (table 25). The exception was for survival through the spillway where the best model expressed $\lambda$ constant across years but $p$ separate across years.

Forebay survival was most related to water temperature, total discharge, and percent spill. The interaction between discharge and percent spill also was significant (table 26). Survival was negatively related to water temperature and predicted survival that ranged from 0.918 to 0.999 while holding other variables at their mean value (table 27, figs. 37 and 38). Furthermore, survival of fish in the forebay was positively related to discharge. Survival predicted by discharge ranged from 0.973 to 0.998 . Survival through the forebay predicted by percent spill ranged from 0.939 to 1.00 . Although percent spill was significant and showed a decrease in survival when it was greater than 60 percent, these high spill values mostly occurred in a single year during 2005, so caution must be exercised when interpreting results.

The best-fit model for fish survival through turbines included water temperature, percent spill, total discharge, and the interaction between percent spill and total discharge (table 28). This was consistent when models included group covariates (for example, photoperiod, passage location). There were other models within two AIC units; however, they included more covariates and did not increase model fit as determined by a likelihood ratio test or examination of the deviance. Survival for subyearling Chinook salmon passing through the turbines was negatively related to temperature and predicted survival estimates ranging from 0.486 to 0.899 (table 27 , fig. 39), but related positively to discharge when percent spill was fixed at its mean value and had predicted estimates ranging from 0.662 to 0.786 when other model covariates were held constant at their mean value (fig. 40). A positive relation between survival and discharge was observed when percent spill was greater than 20 percent. However, when spill was about 20 percent a negative relation was observed. Survival through the turbines also was positively related to percent spill when total discharge was fixed at its mean value, but slopes were small and predicted estimates were within a small range (0.702-0.729). The positive relation between survival and percent spill was apparent as discharge increased to greater than $200 \mathrm{kcfs}$. A negative relation was observed between survival and percent spill when discharge was less than about $200 \mathrm{kcfs}$. Lastly, because discharge had a strong negative correlation to head $(r=-0.832)$, head was substituted for discharge in the model set, however, the best models favored discharge by greater than three AIC units.

For fish that passed McNary Dam through the juvenile bypass system, the best model included only temperature and was consistent with models that included group covariates (table 29). Again, there were other models within two AIC units but they did not contribute significantly to model fit. Survival of fish passing through the bypass system was negatively related to temperature (table 27, fig. 41). The survival estimates predicted by temperature ranged from 0.548 to 0.97 . 
Table 25. Model selection results based on varying $\lambda$ and $p$ with respect to year for subyearling Chinook salmon, 2004-09.

[The best-fit models are indicated in bold. The best-fit model from assessing $\lambda$ was then used to assess the best-fit model for $p$. $\varnothing$, the probability of survival in the forebay or from dam passage through a route to the first downstream detection array; $p$, the probability of being detected at the first downstream detection array given the individual survived; $\lambda$, the joint probability of surviving and being detected from the first downstream array to the next downstream array; y, year]

\begin{tabular}{|c|c|c|c|c|c|}
\hline & Model & $\begin{array}{l}\text { Number of } \\
\text { parameters }\end{array}$ & $\mathrm{AIC} c$ & $\Delta \mathrm{AIC} c$ & Deviance \\
\hline \multicolumn{6}{|c|}{ Forebay } \\
\hline 1 & $\theta(\mathrm{y}) p(\mathrm{y}) \lambda(\mathrm{y})$ & 15 & $7,687.93$ & 0.00 & 0.000 \\
\hline 2 & $\varnothing(\mathrm{y}) p(\mathrm{y}) \lambda$ & 11 & $7,749.94$ & 62.02 & 70.036 \\
\hline 3 & $\varnothing(\mathrm{y}) p \lambda(\mathrm{y})$ & 11 & $7,821.87$ & 133.94 & 141.961 \\
\hline \multicolumn{6}{|c|}{ Turbine } \\
\hline 1 & $\theta(\mathrm{y}) p(\mathrm{y}) \lambda(\mathrm{y})$ & 18 & $4,604.69$ & 0.00 & 0.000 \\
\hline 2 & $\varnothing(\mathrm{y}) p(\mathrm{y}) \lambda$ & 13 & $4,666.00$ & 61.31 & 71.414 \\
\hline 3 & $\varnothing(\mathrm{y}) p \lambda(\mathrm{y})$ & 13 & $4,768.51$ & 163.82 & 173.926 \\
\hline \multicolumn{6}{|c|}{ Bypass } \\
\hline 1 & $\theta(\mathrm{y}) p(\mathrm{y}) \lambda(\mathrm{y})$ & 18 & $4,231.94$ & 0.00 & 0.000 \\
\hline 2 & $\varnothing(\mathrm{y}) p(\mathrm{y}) \lambda$ & 13 & $4,285.00$ & 53.06 & 63.170 \\
\hline 3 & $\varnothing(\mathrm{y}) p \lambda(\mathrm{y})$ & 13 & $4,468.55$ & 236.61 & 246.715 \\
\hline \multicolumn{6}{|c|}{ Spillway } \\
\hline 2 & $\sigma(\mathrm{y}) p(\mathrm{y}) \lambda$ & 11 & $9,944.89$ & 0.00 & 5.167 \\
\hline 1 & $\varnothing(\mathrm{y}) p(\mathrm{y}) \lambda(\mathrm{y})$ & 15 & $9,947.75$ & 2.86 & 0.000 \\
\hline 3 & $\varnothing(\mathrm{y}) p \lambda$ & 7 & $10,699.04$ & 754.149 & 767.334 \\
\hline \multicolumn{6}{|c|}{ Tailrace Releases } \\
\hline 1 & $\sigma(\mathrm{y}) p(\mathrm{y}) \lambda(\mathrm{y})$ & 18 & $13,453.79$ & 0.00 & 0.000 \\
\hline 2 & $\varnothing(\mathrm{y}) p(\mathrm{y}) \lambda$ & 13 & $13,619.47$ & 165.68 & 175.706 \\
\hline 3 & $\varnothing(\mathrm{y}) p \lambda(\mathrm{y})$ & 13 & $14,645.28$ & $1,191.49$ & $1,201.520$ \\
\hline
\end{tabular}



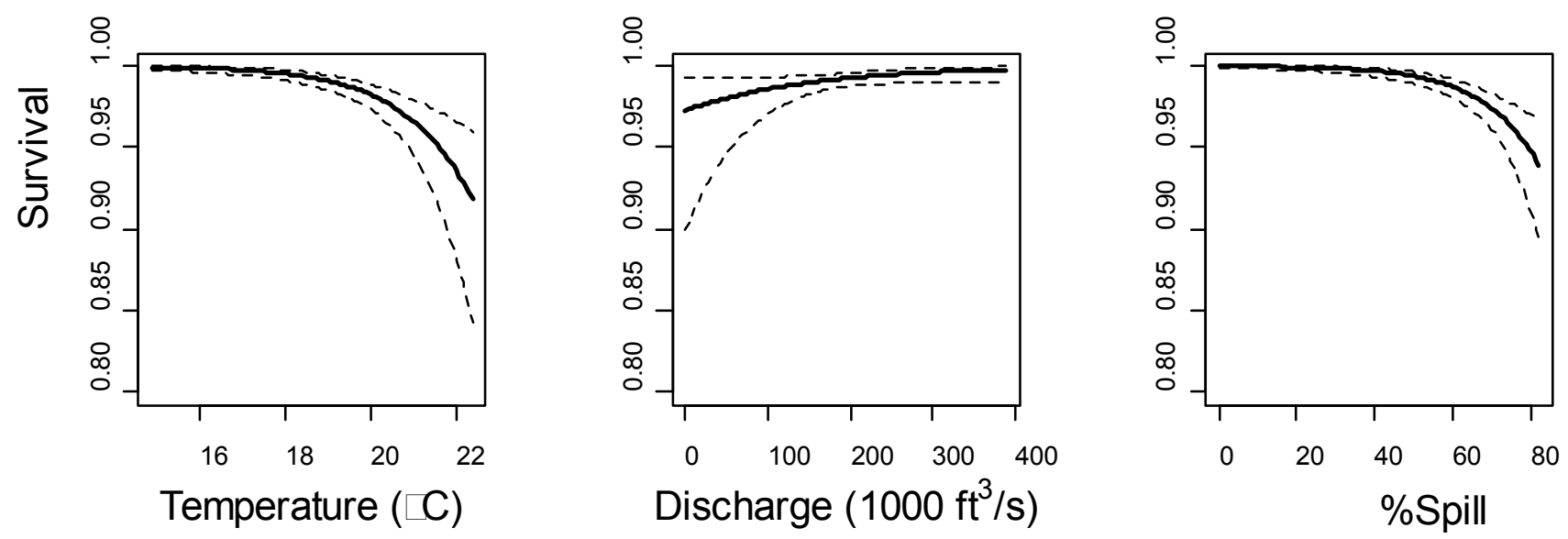

Figure 37. Survival of subyearling Chinook salmon migrating through the forebay of McNary Dam in relation to water temperature, total discharge, and percent spill, 2004-09. Note that most values for percent spill greater than $60 \%$ occurred in a single year during 2005. Other covariates in the model were held constant at their mean value to examine the relationship to the variable of interest. Dashed lines indicate the 95-percent confidence limits. 

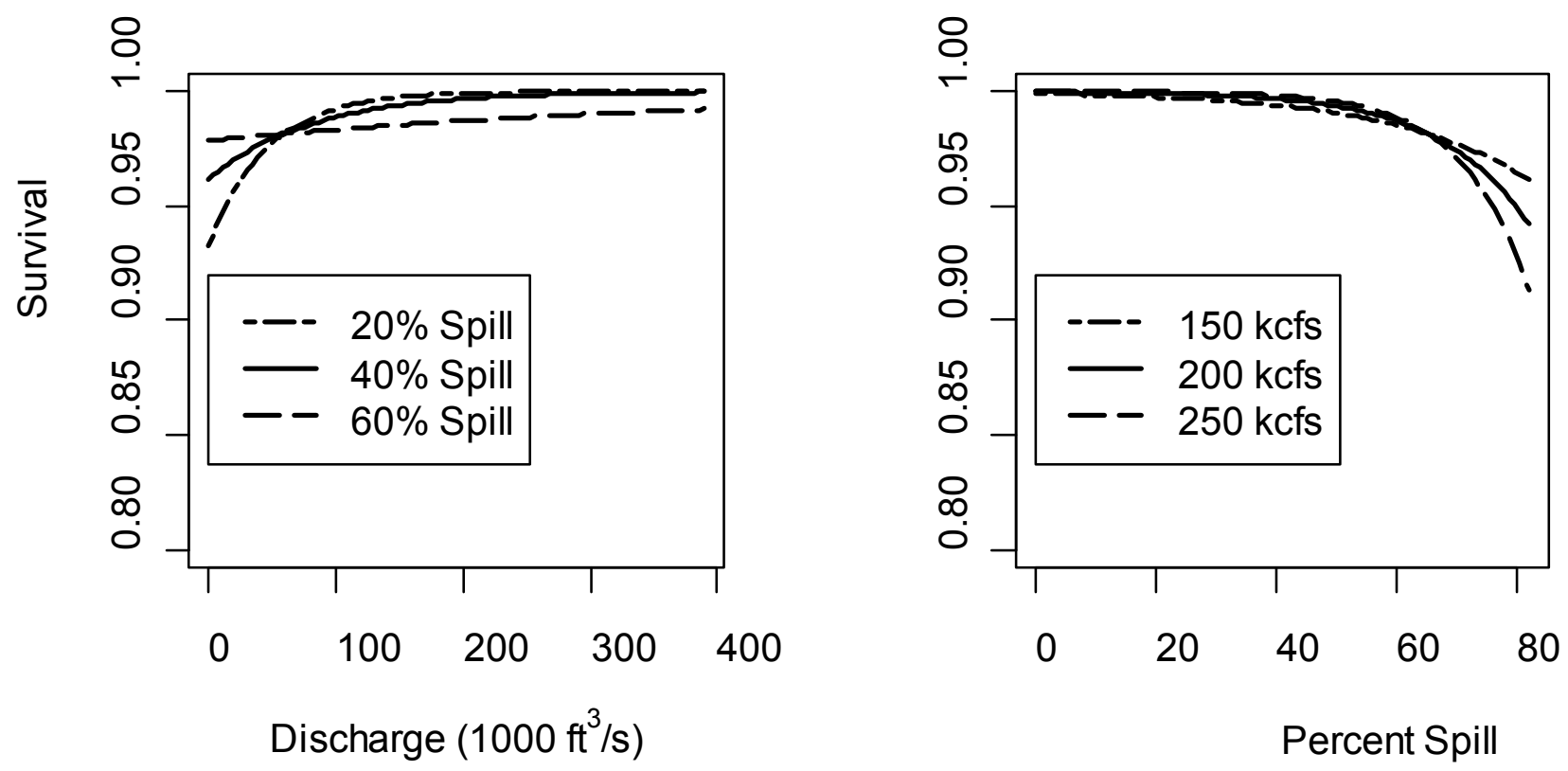

Figure 38. Survival of subyearling Chinook salmon migrating through the forebay of McNary Dam in relation to total discharge, percent spill, and their interaction, 2004-09. Note that most values for percent spill greater than $60 \%$ occurred in a single year during 2005. Other covariates in the model were held constant at their mean value to examine the relationship to the variable of interest. 
Table 26. Model selection for fish that passed through the forebay at McNary Dam relating survival ( $\varnothing)$ as a function of environmental variables using the fixed structure of the best model for $p$ and $\lambda$ parameters for subyearling Chinook salmon, 2004-09.

[The best-fit model is indicated in bold]

\begin{tabular}{|c|c|c|c|c|c|}
\hline Model & $\begin{array}{l}\text { Number of } \\
\text { parameters }\end{array}$ & $\mathrm{AIC} c$ & $\begin{array}{c}\Delta \mathrm{AIC} c \\
\text { (group) }\end{array}$ & $\begin{array}{c}\Delta \mathrm{AIC} c \\
\text { (overall) }\end{array}$ & Deviance \\
\hline Intercept only & 11 & $7,738.13$ & 82.70 & 83.91 & 86.801 \\
\hline Temperature & 12 & $7,675.34$ & 19.90 & 21.12 & $7,651.310$ \\
\hline Discharge & 12 & $7,706.42$ & 50.98 & 52.20 & $7,682.391$ \\
\hline$\%$ Spill & 12 & $7,720.31$ & 64.88 & 66.10 & $7,696.287$ \\
\hline Weight & 12 & $7,738.86$ & 83.43 & 84.64 & $7,714.835$ \\
\hline Temperature $+\%$ Spill & 13 & $7,658.76$ & 3.33 & 4.55 & $7,632.732$ \\
\hline Temperature + Discharge & 13 & $7,675.95$ & 20.51 & 21.73 & $7,649.917$ \\
\hline$\%$ Spill + Discharge & 13 & $7,689.66$ & 34.23 & 35.44 & $7,663.631$ \\
\hline Temperature + Discharge $+\%$ Spill & 14 & $7,658.70$ & 3.27 & 4.48 & $7,630.666$ \\
\hline Weight + Temperature $+\%$ Spill & 14 & $7,660.73$ & 5.29 & 6.51 & $7,632.690$ \\
\hline Weight + Temperature + Discharge & 14 & $7,677.09$ & 21.65 & 22.87 & $7,649.051$ \\
\hline$\%$ Spill + Discharge $+\%$ Spill $\times$ Discharge & 14 & $7,688.14$ & 32.70 & 33.92 & $7,660.100$ \\
\hline Weight $+\%$ Spill + Discharge & 14 & $7,691.50$ & 36.07 & 37.28 & $7,663.465$ \\
\hline Temperature + Discharge $+\%$ Spill $+\%$ Spill $\times$ Discharge & 15 & 7,655.44 & $\mathbf{0 . 0 0}$ & 1.22 & $7,625.394$ \\
\hline Weight + Temperature + Discharge $+\%$ Spill & 15 & $7,660.60$ & 5.16 & 6.38 & $7,630.556$ \\
\hline Weight $+\%$ Spill + Discharge $+\%$ Spill $\times$ Discharge & 15 & $7,689.73$ & 34.30 & 35.51 & $7,659.691$ \\
\hline Weight + Temperature + Discharge $+\%$ Spill $+\%$ Spill $\times$ Discharge & 16 & $7,657.43$ & 1.99 & 3.21 & $7,625.380$ \\
\hline
\end{tabular}


Table 26 (continued). Model selection for fish that passed through the forebay at McNary Dam relating survival ( $\varnothing)$ as a function of environmental variables and group covariates for photoperiod using the fixed structure of the best model for $p$ and $\lambda$ parameters for subyearling Chinook salmon, 2004-09.

\begin{tabular}{|c|c|c|c|c|c|}
\hline Model & $\begin{array}{l}\text { Number of } \\
\text { parameters }\end{array}$ & $\mathrm{AIC} c$ & $\begin{array}{l}\triangle \mathrm{AIC} c \\
\text { (group) }\end{array}$ & $\begin{array}{c}\Delta \mathrm{AIC} c \\
\text { (overall) }\end{array}$ & Deviance \\
\hline Photoperiod & 12 & $7,728.34$ & 74.12 & 74.12 & 75.002 \\
\hline Photoperiod + Temperature & 13 & $7,670.97$ & 16.75 & 16.75 & $7,644.936$ \\
\hline Photoperiod + Discharge & 13 & $7,703.54$ & 49.32 & 49.32 & $7,677.509$ \\
\hline Photoperiod $+\%$ Spill & 13 & $7,712.86$ & 58.65 & 58.65 & $7,686.832$ \\
\hline Photoperiod + Weight & 13 & $7,729.40$ & 75.18 & 75.18 & $7,703.364$ \\
\hline Photoperiod + Weight + Temperature & 14 & $7,672.17$ & 17.96 & 17.96 & $7,644.137$ \\
\hline Photoperiod +\%Spill + Discharge & 14 & $7,687.39$ & 33.17 & 33.17 & $7,659.354$ \\
\hline Photoperiod + Weight + Discharge & 14 & $7,705.54$ & 51.33 & 51.33 & $7,677.507$ \\
\hline Photoperiod + Weight $+\%$ Spill & 14 & $7,712.61$ & 58.39 & 58.39 & $7,684.570$ \\
\hline Photoperiod $+\%$ Spill + Discharge + Temperature & 15 & $7,656.94$ & 2.72 & 2.72 & $7,626.895$ \\
\hline Photoperiod $+\%$ Spill + Discharge $+\%$ Spill $\times$ Discharge & 15 & $7,686.05$ & 31.83 & 31.83 & $7,656.008$ \\
\hline Photoperiod + Weight $+\%$ Spill + Discharge & 15 & $7,689.19$ & 34.97 & 34.97 & $7,659.145$ \\
\hline Photoperiod $+\%$ Spill + Discharge + Temperature $+\%$ Spill $\times$ Discharge & 16 & $7,654.22$ & 0.00 & 0.00 & $7,622.171$ \\
\hline Photoperiod + Weight $+\%$ Spill + Discharge + Temperature & 16 & $7,658.90$ & 4.68 & 4.68 & $7,626.852$ \\
\hline Photoperiod + Weight $+\%$ Spill + Discharge $+\%$ Spill $\times$ Discharge & 16 & $7,687.64$ & 33.42 & 33.42 & $7,655.591$ \\
\hline Photoperiod + Weight $+\%$ Spill + Discharge + Temperature $+\%$ Spill $\times$ Discharge & 17 & $7,656.22$ & 2.00 & 2.00 & $7,622.170$ \\
\hline
\end{tabular}


Table 27. Slope (Beta) coefficients, standard error, and 95-percent confidence limits of model parameters for subyearling Chinook salmon passing McNary Dam, 2004-09.

[CL, confidence limit]

\begin{tabular}{|c|c|c|c|c|}
\hline Variable & Beta & $\begin{array}{r}\text { Standard } \\
\text { error }\end{array}$ & Lower CL & Upper CL \\
\hline \multicolumn{5}{|c|}{ Forebay } \\
\hline Intercept & 14.194 & 3.018 & 8.279 & 20.109 \\
\hline Temperature & -0.667 & 0.131 & -0.923 & -0.411 \\
\hline Discharge & 0.034 & 0.011 & 0.014 & 0.055 \\
\hline$\%$ Spill & 0.032 & 0.029 & -0.025 & 0.089 \\
\hline Discharge $\times \%$ Spill & -0.0005 & 0.0001 & -0.0009 & -0.0002 \\
\hline \multicolumn{5}{|c|}{ Turbine } \\
\hline Intercept & 7.197 & 1.259 & 4.730 & 9.665 \\
\hline Temperature & -0.299 & 0.052 & -0.401 & -0.197 \\
\hline Discharge & -0.003 & 0.003 & -0.010 & 0.003 \\
\hline$\%$ Spill & -0.026 & 0.011 & -0.048 & -0.005 \\
\hline Discharge $\times \%$ Spill & 0.000148 & 0.0000689 & 0.0000133 & 0.000284 \\
\hline \multicolumn{5}{|c|}{ Bypass } \\
\hline Intercept & 10.350 & 1.220 & 7.958 & 12.742 \\
\hline Temperature & -0.458 & 0.062 & -0.580 & -0.336 \\
\hline \multicolumn{5}{|c|}{ Spillway } \\
\hline Intercept & 5.266 & 1.296 & 2.727 & 7.806 \\
\hline Tag Burdens & -0.192 & 0.063 & -0.316 & -0.068 \\
\hline Temperature & -0.240 & 0.053 & -0.344 & -0.137 \\
\hline Discharge & 0.011 & 0.002 & 0.007 & 0.014 \\
\hline$\%$ Spill & 0.004 & 0.005 & -0.006 & 0.014 \\
\hline \multicolumn{5}{|c|}{ Tailrace Releases } \\
\hline Intercept & 9.124 & 0.808 & 7.541 & 10.708 \\
\hline Temperature & -0.372 & 0.040 & -0.451 & -0.293 \\
\hline Photoperiod (light) & 0.300 & 0.132 & 0.041 & 0.558 \\
\hline
\end{tabular}


Table 28. Model selection for fish that passed through turbines at McNary Dam relating survival $(\varnothing)$ as a function of environmental variables using the fixed structure of the best model for $p$ and $\lambda$ parameters for subyearling Chinook salmon, 2004-09.

[The best-fit model is indicated in bold]

\begin{tabular}{|c|c|c|c|c|c|}
\hline Model & $\begin{array}{l}\text { Number of } \\
\text { parameters }\end{array}$ & $\mathrm{AIC} c$ & $\begin{array}{c}\Delta \mathrm{AIC} c \\
\text { (group) }\end{array}$ & $\begin{array}{c}\Delta \mathrm{AlC} c \\
\text { (overall) }\end{array}$ & Deviance \\
\hline Intercept only & 13 & $4,606.15$ & 91.85 & 91.85 & 108.526 \\
\hline Weight & 14 & $4,606.06$ & 91.77 & 91.77 & $4,577.923$ \\
\hline Discharge & 14 & $4,551.19$ & 36.89 & 36.89 & $4,523.049$ \\
\hline$\%$ Spill & 14 & $4,604.79$ & 90.49 & 90.49 & $4,576.650$ \\
\hline Temperature & 14 & $4,516.87$ & 2.58 & 2.58 & $4,488.733$ \\
\hline$\%$ Spill + Discharge & 15 & $4,551.07$ & 36.77 & 36.77 & $4,520.910$ \\
\hline Temperature + Discharge & 15 & $4,517.02$ & 2.72 & 2.72 & $4,486.860$ \\
\hline Temperature $+\%$ Spill & 15 & $4,518.28$ & 3.99 & 3.99 & $4,488.127$ \\
\hline Weight $+\%$ Spill + Discharge & 16 & $4,552.83$ & 38.54 & 38.54 & $4,520.656$ \\
\hline Weight + Temperature + Discharge & 16 & $4,516.33$ & 2.03 & 2.03 & $4,484.149$ \\
\hline Weight + Temperature $+\%$ Spill & 16 & $4,518.51$ & 4.22 & 4.22 & $4,486.335$ \\
\hline Temperature + Discharge $+\%$ Spill & 16 & $4,517.20$ & 2.91 & 2.91 & $4,485.027$ \\
\hline$\%$ Spill + Discharge $+\%$ Spill $\times$ Discharge & 16 & $4,547.73$ & 33.44 & 33.44 & $4,515.553$ \\
\hline Weight + Temperature + Discharge $+\%$ Spill & 17 & $4,517.13$ & 2.84 & 2.84 & $4,482.932$ \\
\hline Weight $+\%$ Spill + Discharge $+\%$ Spill $\times$ Discharge & 17 & $4,549.55$ & 35.25 & 35.25 & $4,515.347$ \\
\hline Temperature + Discharge $+\%$ Spill $+\%$ Spill $\times$ Discharge & 17 & 4,514.46 & 0.16 & 0.16 & $4,480.256$ \\
\hline Weight + Temperature + Discharge $+\%$ Spill $+\%$ Spill $\times$ Discharge & 18 & $4,514.29$ & 0.00 & 0.00 & $4,478.071$ \\
\hline
\end{tabular}


Table 28 (continued). Model selection for fish that passed through turbines at McNary Dam relating survival ( $\varnothing)$ as a function of environmental variables and group covariates for photoperiod using the fixed structure of the best model for $p$ and $\lambda$ parameters for subyearling Chinook salmon, 2004-09.

\begin{tabular}{|c|c|c|c|c|c|}
\hline Model & $\begin{array}{l}\text { Number of } \\
\text { parameters }\end{array}$ & $\mathrm{AIC} c$ & $\begin{array}{l}\Delta \mathrm{AIC} c \\
\text { (group) }\end{array}$ & $\begin{array}{c}\Delta \mathrm{AIC} c \\
\text { (overall) }\end{array}$ & Deviance \\
\hline Photoperiod & 14 & $4,603.29$ & 87.06 & 89.00 & 103.651 \\
\hline Photoperiod + Discharge & 15 & $4,552.88$ & 36.65 & 38.59 & $4,522.725$ \\
\hline Photoperiod + \%Spill & 15 & $4,602.56$ & 86.33 & 88.27 & $4,572.405$ \\
\hline Photoperiod + Weight & 15 & $4,603.81$ & 87.58 & 89.51 & $4,573.652$ \\
\hline Photoperiod + Temperature & 15 & $4,518.88$ & 2.65 & 4.59 & $4,488.724$ \\
\hline Photoperiod + Weight $+\%$ Spill & 16 & $4,603.87$ & 87.64 & 89.58 & $4,571.696$ \\
\hline Photoperiod + Weight + Discharge & 16 & $4,554.38$ & 38.15 & 40.08 & $4,522.200$ \\
\hline Photoperiod $+\%$ Spill + Discharge & 16 & $4,552.61$ & 36.38 & 38.32 & $4,520.436$ \\
\hline Photoperiod + Weight + Temperature & 16 & $4,518.75$ & 2.52 & 4.45 & $4,486.569$ \\
\hline Photoperiod + Weight $+\%$ Spill + Discharge & 17 & $4,554.39$ & 38.16 & 40.10 & $4,520.190$ \\
\hline Photoperiod $+\%$ Spill + Discharge + Temperature & 17 & $4,519.02$ & 2.79 & 4.73 & $4,484.821$ \\
\hline Photoperiod $+\%$ Spill + Discharge $+\%$ Spill $\times$ Discharge & 17 & $4,549.50$ & 33.27 & 35.20 & $4,515.297$ \\
\hline Photoperiod + Weight $+\%$ Spill + Discharge + Temperature & 18 & $4,518.96$ & 2.72 & 4.66 & $4,482.733$ \\
\hline Photoperiod + Weight $+\%$ Spill + Discharge $+\%$ Spill $\times$ Discharge & 18 & $4,551.32$ & 35.09 & 37.03 & $4,515.097$ \\
\hline Photoperiod $+\%$ Spill + Discharge + Temperature $+\%$ Spill $\times$ Discharge & 18 & $4,516.38$ & 0.15 & 2.09 & $4,480.161$ \\
\hline Photoperiod + Weight $+\%$ Spill + Discharge + Temperature $+\%$ Spill $\times$ Discharge & 19 & $4,516.23$ & 0.00 & 1.94 & 4,477.984 \\
\hline
\end{tabular}


Table 28 (continued). Model selection for fish that passed through turbines at McNary Dam relating survival $(\varnothing)$ as a function of environmental variables and group covariates for turbine passage location (i.e. north, south) using the fixed structure of the best model for $p$ and $\lambda$ parameters for subyearling Chinook salmon, 2004-09.

\begin{tabular}{|c|c|c|c|c|c|}
\hline Model & $\begin{array}{l}\text { Number of } \\
\text { parameters }\end{array}$ & $\mathrm{AIC} c$ & $\begin{array}{l}\Delta \mathrm{AIC} c \\
\text { (group) }\end{array}$ & $\begin{array}{c}\Delta \mathrm{AIC} c \\
\text { (overall) }\end{array}$ & Deviance \\
\hline TurbPassLoc & 14 & $4,607.53$ & 91.31 & 93.24 & 107.891 \\
\hline TurbPassLoc + Temperature & 15 & $4,518.86$ & 2.64 & 4.56 & $4,488.700$ \\
\hline TurbPassLoc + Discharge & 15 & $4,553.17$ & 36.96 & 38.88 & $4,523.019$ \\
\hline TurbPassLoc $+\%$ Spill & 15 & $4,606.56$ & 90.34 & 92.27 & $4,576.406$ \\
\hline TurbPassLoc + Weight & 15 & $4,607.53$ & 91.31 & 93.24 & $4,577.373$ \\
\hline TurbPassLoc $+\%$ Spill + Discharge & 16 & $4,553.09$ & 36.87 & 38.79 & $4,520.909$ \\
\hline TurbPassLoc + Temperature + Discharge & 16 & $4,519.03$ & 2.82 & 4.74 & $4,486.858$ \\
\hline TurbPassLoc + Weight $+\%$ Spill + Discharge & 17 & $4,554.85$ & 38.64 & 40.56 & $4,520.655$ \\
\hline TurbPassLoc + Temperature $+\%$ Spill & 16 & $4,520.21$ & 3.99 & 5.91 & 4,488.032 \\
\hline TurbPassLoc + Weight + Temperature + Discharge & 17 & $4,518.34$ & 2.12 & 4.05 & $4,484.143$ \\
\hline TurbPassLoc + Temperature + Discharge $+\%$ Spill & 17 & $4,519.17$ & 2.96 & 4.88 & $4,484.975$ \\
\hline TurbPassLoc $+\%$ Spill + Discharge $+\%$ Spill $\times$ Discharge & 17 & $4,549.74$ & 33.53 & 35.45 & $4,515.546$ \\
\hline TurbPassLoc + Weight + Temperature + Discharge $+\%$ Spill & 18 & $4,519.11$ & 2.89 & 4.81 & $4,482.883$ \\
\hline TurbPassLoc + Weight $+\%$ Spill + Discharge $+\%$ Spill $\times$ Discharge & 18 & $4,551.56$ & 35.35 & 37.27 & $4,515.341$ \\
\hline TurbPassLoc + Temperature + Discharge $+\%$ Spill $+\%$ Spill $\times$ Discharge & 18 & $4,516.38$ & 0.16 & 2.08 & $4,480.153$ \\
\hline TurbPassLoc + Weight + Temperature + Discharge $+\%$ Spill $+\%$ Spill $\times$ Discharge & 19 & $4,516.22$ & 0.00 & 1.92 & $4,477.970$ \\
\hline
\end{tabular}


Table 28 (continued). Model selection for fish that passed through turbines at McNary Dam relating survival (ø) as a function of environmental variables and group covariates for turbine passage location (i.e. north, south) and photoperiod using the fixed structure of the best model for $p$ and $\lambda$ parameters for subyearling Chinook salmon, 2004-09.

\begin{tabular}{|c|c|c|c|c|c|}
\hline Model & $\begin{array}{l}\text { Number of } \\
\text { parameters }\end{array}$ & $\mathrm{AIC}_{c}$ & $\begin{array}{l}\Delta \mathrm{AIC} c \\
\text { (group) }\end{array}$ & $\begin{array}{c}\Delta \mathrm{AIC} c \\
\text { (overall) }\end{array}$ & Deviance \\
\hline TurbPassLoc + Photoperiod & 15 & $4,604.84$ & 86.68 & 90.54 & 103.179 \\
\hline TurbPassLoc + Photoperiod + Weight & 16 & $4,605.41$ & 87.25 & 91.11 & $4,573.230$ \\
\hline TurbPassLoc + Photoperiod + Discharge & 16 & $4,554.87$ & 36.71 & 40.58 & $4,522.692$ \\
\hline TurbPassLoc + Photoperiod $+\%$ Spill & 16 & $4,604.39$ & 86.24 & 90.10 & $4,572.218$ \\
\hline TurbPassLoc + Photoperiod + Weight + Discharge & 17 & $4,556.37$ & 38.21 & 42.08 & $4,522.171$ \\
\hline TurbPassLoc + Photoperiod + Temperature & 16 & $4,520.87$ & 2.71 & 6.57 & $4,488.691$ \\
\hline TurbPassLoc + Photoperiod + Weight $+\%$ Spill & 17 & $4,605.70$ & 87.55 & 91.41 & $4,571.506$ \\
\hline TurbPassLoc + Photoperiod $+\%$ Spill + Discharge & 17 & $4,554.63$ & 36.48 & 40.34 & $4,520.436$ \\
\hline TurbPassLoc + Photoperiod + Weight + Temperature & 17 & $4,520.72$ & 2.56 & 6.42 & $4,486.519$ \\
\hline TurbPassLoc + Photoperiod + Weight $+\%$ Spill + Discharge & 18 & $4,556.41$ & 38.25 & 42.12 & $4,520.190$ \\
\hline TurbPassLoc + Photoperiod $+\%$ Spill + Discharge + Temperature & 18 & $4,520.99$ & 2.84 & 6.70 & $4,484.770$ \\
\hline TurbPassLoc + Photoperiod $+\%$ Spill + Discharge $+\%$ Spill $\times$ Discharge & 18 & $4,551.51$ & 33.36 & 37.22 & $4,515.290$ \\
\hline TurbPassLoc + Photoperiod + Weight $+\%$ Spill + Discharge + Temperature & 19 & $4,520.93$ & 2.77 & 6.64 & $4,482.684$ \\
\hline TurbPassLoc + Photoperiod + Weight $+\%$ Spill + Discharge $+\%$ Spill $\times$ Discharge & 19 & $4,553.34$ & 35.18 & 39.04 & $4,515.091$ \\
\hline TurbPassLoc + Photoperiod $+\%$ Spill + Discharge + Temperature $+\%$ Spill $\times$ Discharge & 19 & $4,518.31$ & 0.15 & 4.01 & $4,480.059$ \\
\hline TurbPassLoc + Photoperiod + Weight $+\%$ Spill + Discharge + Temperature $+\%$ Spill $\times$ Discharge & 20 & $4,518.16$ & 0.00 & 3.86 & $4,477.884$ \\
\hline
\end{tabular}




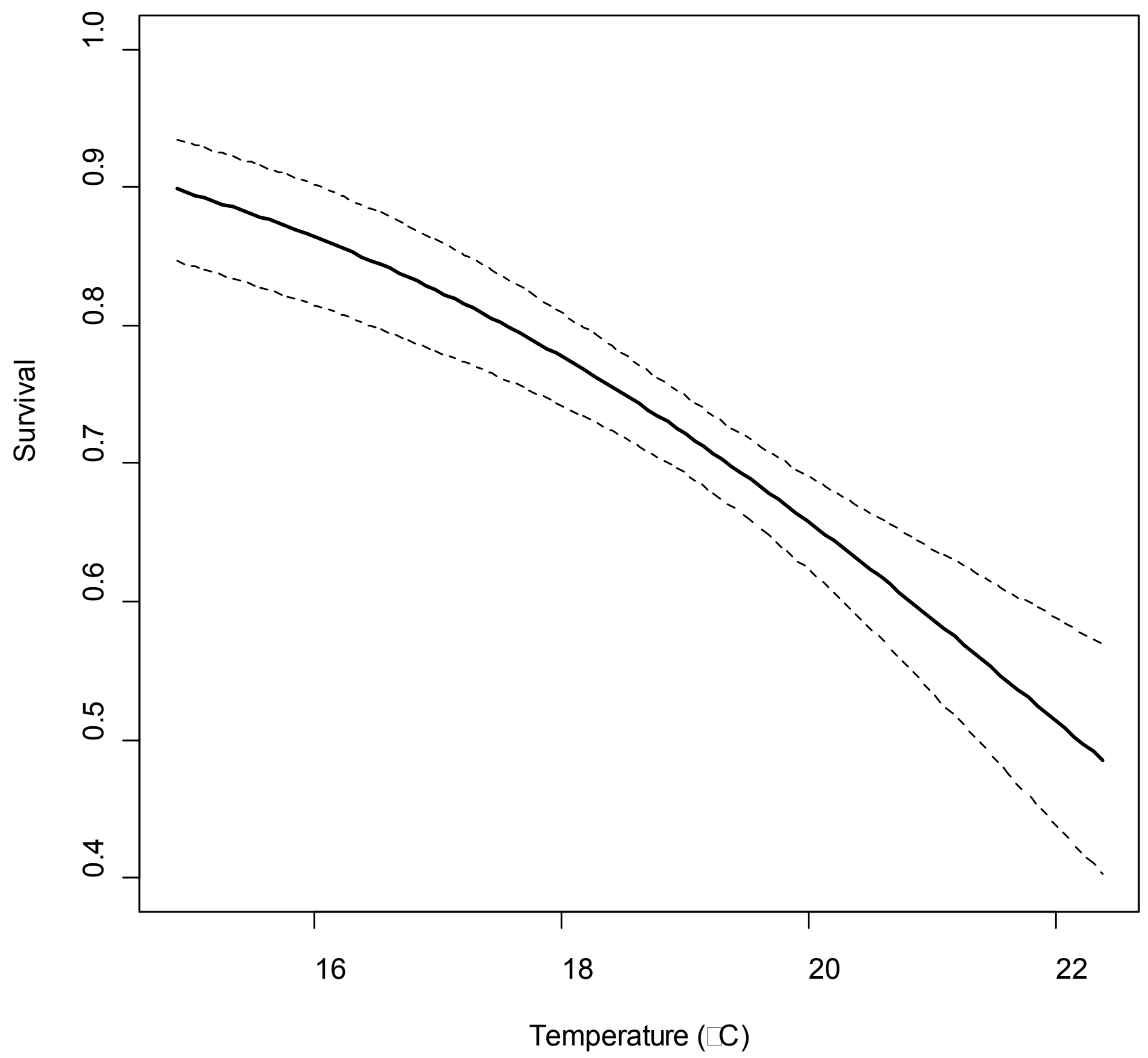

Figure 39. Survival of subyearling Chinook salmon passing through turbines at McNary Dam in relation to water temperature, 2004-09. The dashed lines represent the 95-percent confidence limits. Other covariates in the model were held constant at their mean value to examine the relationship to the variable of interest. 

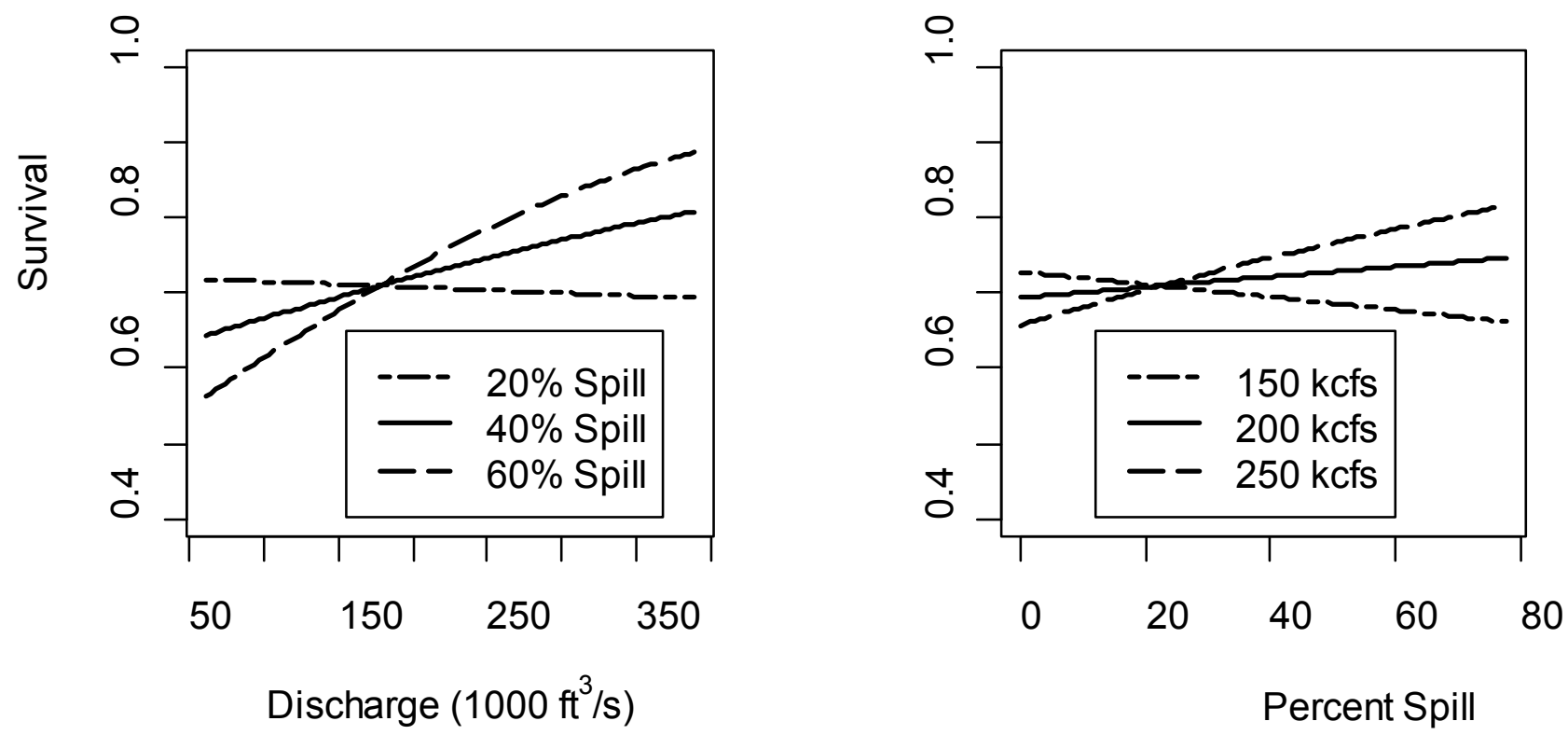

Figure 40. Survival of subyearling Chinook salmon passing through turbines at McNary Dam in relation to percent spill and discharge, 2004-09. Other covariates in the model were held constant at their mean value to examine the relationship to the variable of interest. 
Table 29. Model selection for fish that passed through the juvenile bypass system at McNary Dam relating survival $(\varnothing)$ as a function of environmental variables using the fixed structure of the best model for $p$ and $\lambda$ parameters for subyearling Chinook salmon, 2004-09.

[The best-fit model is indicated in bold]

\begin{tabular}{|c|c|c|c|c|c|}
\hline Model & $\begin{array}{c}\text { Number of } \\
\text { parameters }\end{array}$ & $\mathrm{AIC} c$ & $\begin{array}{c}\Delta \mathrm{AIC} c \\
\text { (group) }\end{array}$ & $\begin{array}{c}\Delta \mathrm{AIC} c \\
\text { (overall) }\end{array}$ & Deviance \\
\hline Intercept only & 13 & $4,249.56$ & 71.34 & 71.34 & 46.159 \\
\hline Temperature & 14 & $4,179.34$ & 1.12 & 1.12 & $4,151.205$ \\
\hline Discharge & 14 & $4,212.64$ & 34.42 & 34.42 & $4,184.499$ \\
\hline$\%$ Spill & 14 & $4,234.69$ & 56.47 & 56.47 & $4,206.554$ \\
\hline Weight & 14 & $4,248.88$ & 70.66 & 70.66 & $4,220.744$ \\
\hline Temperature $+\%$ Spill & 15 & $4,178.62$ & 0.40 & 0.40 & $4,148.461$ \\
\hline Temperature + Discharge & 15 & $4,180.49$ & 2.27 & 2.27 & $4,150.332$ \\
\hline$\%$ Spill + Discharge & 15 & $4,211.51$ & 33.29 & 33.29 & $4,181.354$ \\
\hline Weight + Temperature $+\%$ Spill & 16 & $4,178.22$ & 0.00 & 0.00 & $4,146.040$ \\
\hline Temperature + Discharge $+\%$ Spill & 16 & $4,180.52$ & 2.30 & 2.30 & $4,148.337$ \\
\hline Weight + Temperature + Discharge & 16 & $4,181.26$ & 3.04 & 3.04 & $4,149.076$ \\
\hline$\%$ Spill + Discharge $+\%$ Spill $\times$ Discharge & 16 & $4,211.51$ & 33.29 & 33.29 & $4,179.333$ \\
\hline Weight $+\%$ Spill + Discharge & 16 & $4,213.47$ & 35.25 & 35.25 & $4,181.288$ \\
\hline Weight + Temperature + Discharge $+\%$ Spill & 17 & $4,180.09$ & 1.87 & 1.87 & $4,145.892$ \\
\hline Temperature + Discharge $+\%$ Spill $+\%$ Spill $\times$ Discharge & 17 & $4,181.60$ & 3.38 & 3.38 & $4,147.400$ \\
\hline Weight $+\%$ Spill + Discharge $+\%$ Spill $\times$ Discharge & 17 & $4,213.40$ & 35.18 & 35.18 & $4,179.201$ \\
\hline Weight + Temperature + Discharge $+\%$ Spill $+\%$ Spill $\times$ Discharge & 18 & $4,181.37$ & 3.14 & 3.14 & $4,145.138$ \\
\hline
\end{tabular}


Table 29 (continued). Model selection for fish that passed through the juvenile bypass system at McNary Dam relating survival (ø) as a function of environmental variables and group covariates for photoperiod using the fixed structure of the best model for $p$ and $\lambda$ parameters for subyearling Chinook salmon, 2004-09.

\begin{tabular}{|c|c|c|c|c|c|}
\hline Model & $\begin{array}{l}\text { Number of } \\
\text { parameters }\end{array}$ & $\mathrm{AIC} c$ & $\begin{array}{l}\Delta \mathrm{AIC} c \\
\text { (group) }\end{array}$ & $\begin{array}{c}\Delta \mathrm{AIC} c \\
\text { (overall) }\end{array}$ & Deviance \\
\hline Photoperiod & 14 & $4,251.22$ & 69.86 & 73.00 & 45.796 \\
\hline Photoperiod + Discharge & 15 & $4,213.97$ & 32.62 & 35.75 & $4,183.812$ \\
\hline Photoperiod $+\%$ Spill & 15 & $4,236.25$ & 54.90 & 58.03 & $4,206.094$ \\
\hline Photoperiod + Temperature & 15 & $4,181.36$ & 0.00 & 3.13 & $4,151.196$ \\
\hline Photoperiod + Weight & 15 & $4,250.50$ & 69.14 & 72.28 & $4,220.339$ \\
\hline Photoperiod $+\%$ Spill + Discharge & 16 & $4,213.17$ & 31.81 & 34.94 & $4,180.985$ \\
\hline Photoperiod + Weight + Temperature & 16 & $4,182.39$ & 1.04 & 4.17 & $4,150.212$ \\
\hline Photoperiod + Weight + Discharge & 16 & $4,215.98$ & 34.63 & 37.76 & $4,183.803$ \\
\hline Photoperiod + Weight $+\%$ Spill & 16 & $4,237.89$ & 56.53 & 59.67 & $4,205.709$ \\
\hline Photoperiod + Weight $+\%$ Spill + Discharge & 17 & $4,215.11$ & 33.75 & 36.89 & $4,180.905$ \\
\hline Photoperiod $+\%$ Spill + Discharge $+\%$ Spill $\times$ Discharge & 17 & $4,213.21$ & 31.85 & 34.99 & $4,179.005$ \\
\hline Photoperiod $+\%$ Spill + Discharge + Temperature & 17 & $4,182.53$ & 1.17 & 4.30 & $4,148.322$ \\
\hline Photoperiod + Weight $+\%$ Spill + Discharge + Temperature & 18 & $4,182.09$ & 0.73 & 3.87 & $4,145.859$ \\
\hline Photoperiod $+\%$ Spill + Discharge + Temperature $+\%$ Spill $\times$ Discharge & 18 & $4,183.62$ & 2.26 & 5.40 & $4,147.391$ \\
\hline Photoperiod + Weight $+\%$ Spill + Discharge $+\%$ Spill $\times$ Discharge & 18 & $4,215.09$ & 33.73 & 36.87 & $4,178.860$ \\
\hline Photoperiod + Weight $+\%$ Spill + Discharge + Temperature $+\%$ Spill $\times$ Discharge & 19 & $4,183.37$ & 2.01 & 5.15 & $4,145.117$ \\
\hline
\end{tabular}




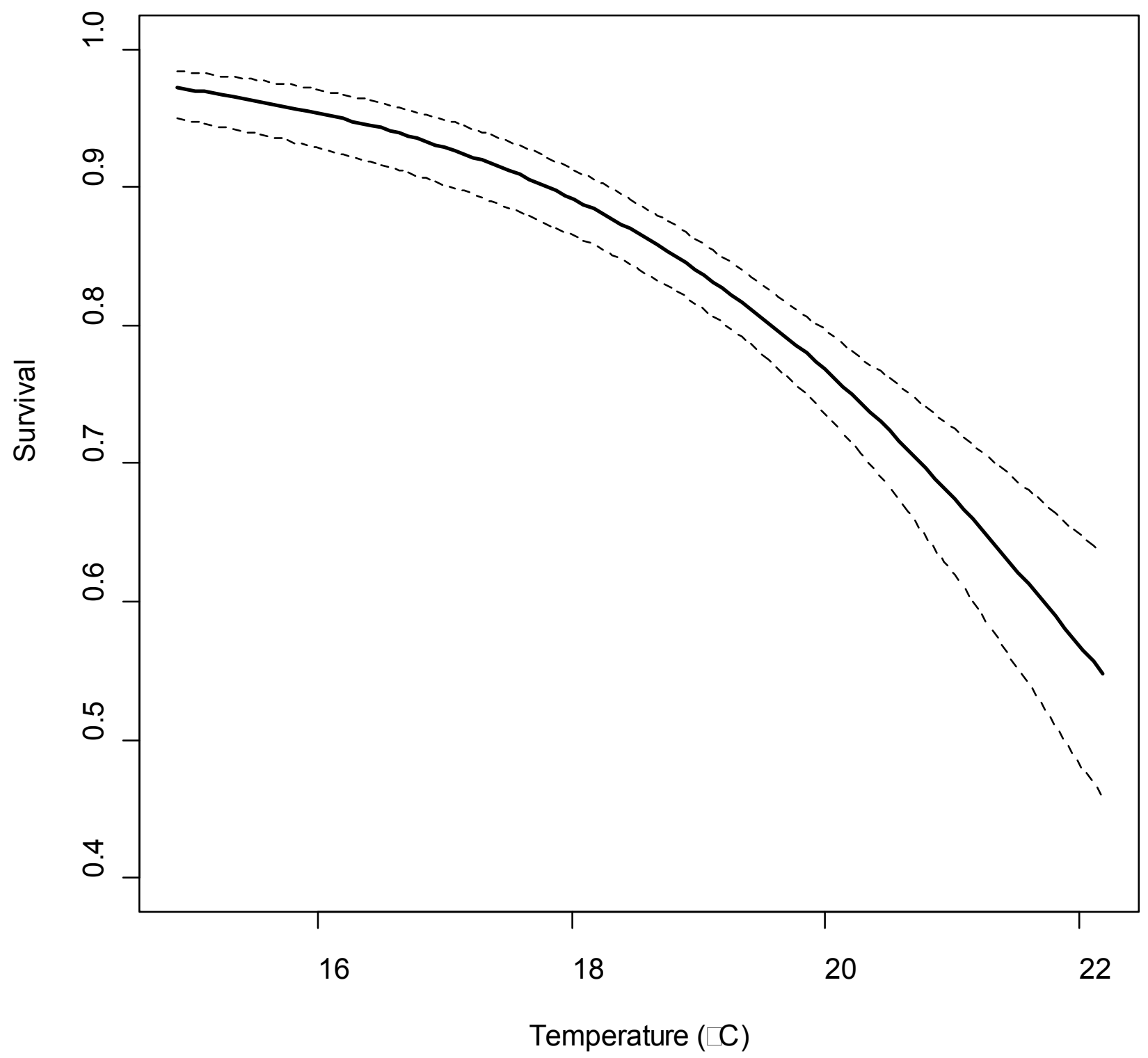

Figure 41. Survival of subyearling Chinook salmon passing through the juvenile bypass system at McNary Dam in relation to water temperature, 2004-09. The dashed lines represent the 95-percent confidence limits. 
The best model for describing survival for fish passage through the spillway included fish weight, water temperature, total discharge, and percent spill (table 30). Models that included group covariates were consistent with this pattern, except for models containing group covariates for passage location (that is, north vs. south). In this instance, percent spill was significant at the $\alpha$ level of 0.10 , but was not significant at the $\alpha$ level of 0.05 determined by a likelihood ratio test. Survival of fish through the spillway was negatively related to temperature and ranged from 0.819 to 0.964 when other model covariates were held constant at their mean value, but was not as strong as a relation observed for fish passing through the juvenile bypass system or turbine routes (table 27). Predicted survival was higher at the lower end of the range for water temperatures and lower at higher temperatures. Survival through the spillway was positively related to discharge and percent spill, the stronger relation being to discharge predicting survival estimates ranging from 0.819 to 0.964 (fig. 42). The slope for survival through the spillway versus percent spill was fairly flat and was not significantly different than zero (table 27, fig. 42). This indicates that percent spill was less important in explaining variation of survival estimates. Because fish weight was an important variable in describing variation of survival of fish through the spillway, variables highly correlated (for example, tag burden) with fish weight were substituted for fish weight in the best model. The model with tag burden fit the data significantly better than the model with fish weight. The model with tag burden had an AIC value 4 units lower than the model with fish weight; therefore, tag burden was used to predict survival when examining relations between spillway survival and important environmental variables.

Survival for fish released in the tailrace of McNary Dam were best fit with the model that included water temperature and photoperiod (table 31). Survival was slightly higher during the day than the night photoperiod. Note there were only releases in the tailrace during the night photoperiod for the years 2004, 2005, and 2009. Therefore, this difference could be related to changes across years, and not due to photoperiod, especially if conditions in years 2006-08 were different than years 2004, 2005, and 2009. Survival predicted by photoperiod and temperature of tailrace released fish ranged from 0.704 to 0.980 and was negatively related to temperature. This was similar to the relation between survival through the spillway and temperature (table 27, fig. 43). Release location was also tested to determine if there was a difference in survival among fish released in the north, middle, or south portion of the channel in the tailrace and there was no statistical difference.

A year factor was added to the best model for survival of subyearling Chinook salmon for each route to determine if it would explain any additional variation in survival. Adding a year effect significantly increased model fit by decreasing AIC by at least 5 units, except for the turbine route. For fish passing the turbine route, adding a year effect did not increase model fit. The best model without year regarding model fit was at least 5 AIC units better than the model with year included. A factor indicating whether a TSW was installed during a particular year also was included in place of year when survival through the spillway and forebay was examined. The model with a TSW factor fit significantly better than the best model with a year factor and resulted in decreasing AIC by $>3$ units and showed survival through the spillway being negatively related to the TSW factor $\left(\beta_{\mathrm{TSW}}=-0.569, \mathrm{LCL}=-0.846\right.$, $\mathrm{UCL}=-0.291$ ). Adding a TSW factor variable for survival in the forebay was not significant, however, adding a year factor when modeling forebay survival was significant. The TSW factor is better thought of as a modified year effect where the TSW factor equals ' 0 ' during 2004-06, and ' 1 ' during 2007-09, because it is confounded by inter-annual differences. Therefore, it should not be thought of strictly as an effect of having a TSW installed. Instead, it should be thought of as representing all differences between the years when the TSW was and was not installed at McNary Dam. As a result, caution should be exercised when interpreting the results from the models that included the TSW factor. 
Table 30. Model selection for fish that passed through the spillway at McNary Dam relating survival ( $\varnothing$ ) as a function of environmental variables using the fixed structure of the best model for $p$ and $\lambda$ parameters for subyearling Chinook salmon, 2004-09.

[The best-fit model is indicated in bold]

\begin{tabular}{|c|c|c|c|c|c|}
\hline Model & $\begin{array}{l}\text { Number of } \\
\text { parameters }\end{array}$ & $\mathrm{AIC} c$ & $\begin{array}{c}\Delta \mathrm{AIC} c \\
\text { (group) }\end{array}$ & $\begin{array}{c}\Delta \mathrm{AIC} c \\
\text { (overall) }\end{array}$ & Deviance \\
\hline Intercept only & 7 & $9,977.93$ & 139.63 & 139.63 & 138.419 \\
\hline Discharge & 8 & $9,855.03$ & 16.73 & 16.73 & $9,839.017$ \\
\hline Temperature & 8 & $9,885.28$ & 46.97 & 46.97 & $9,869.259$ \\
\hline Weight & 8 & $9,979.87$ & 141.56 & 141.56 & $9,963.850$ \\
\hline$\%$ Spill & 8 & $9,979.92$ & 141.62 & 141.62 & $9,963.903$ \\
\hline Temperature + Discharge & 9 & $9,841.59$ & 3.28 & 3.28 & $9,823.564$ \\
\hline$\%$ Spill + Discharge & 9 & $9,855.79$ & 17.49 & 17.49 & $9,837.767$ \\
\hline Temperature $+\%$ Spill & 9 & $9,885.01$ & 46.70 & 46.70 & $9,866.985$ \\
\hline Weight + Temperature + Discharge & 10 & $9,840.19$ & 1.88 & 1.88 & $9,820.159$ \\
\hline Temperature + Discharge $+\%$ Spill & 10 & $9,841.64$ & 3.33 & 3.33 & $9,821.609$ \\
\hline Weight $+\%$ Spill + Discharge & 10 & $9,855.43$ & 17.13 & 17.13 & $9,835.407$ \\
\hline$\%$ Spill + Discharge $+\%$ Spill $\times$ Discharge & 10 & $9,857.68$ & 19.38 & 19.38 & $9,837.655$ \\
\hline Weight + Temperature $+\%$ Spill & 10 & $9,882.28$ & 43.98 & 43.98 & $9,862.257$ \\
\hline Weight + Temperature + Discharge $+\%$ Spill & 11 & $9,838.30$ & 0.00 & 0.00 & $9,816.272$ \\
\hline Temperature + Discharge $+\%$ Spill $+\%$ Spill $\times$ Discharge & 11 & $9,843.57$ & 5.27 & 5.27 & $9,821.537$ \\
\hline Weight $+\%$ Spill + Discharge $+\%$ Spill $\times$ Discharge & 11 & $9,857.32$ & 19.02 & 19.02 & $9,835.289$ \\
\hline Weight + Temperature + Discharge $+\%$ Spill $+\%$ Spill $\times$ Discharge & 12 & $9,840.24$ & 1.94 & 1.94 & $9,816.204$ \\
\hline
\end{tabular}


Table 30 (continued). Model selection for fish that passed through the spillway at McNary Dam relating survival ( $\varnothing$ ) as a function of environmental variables and group covariates for photoperiod using the fixed structure of the best model for $p$ and $\lambda$ parameters for subyearling Chinook salmon, 2004-09.

\begin{tabular}{|c|c|c|c|c|c|}
\hline Model & $\begin{array}{c}\text { Number of } \\
\text { parameters }\end{array}$ & $\mathrm{AIC} c$ & $\begin{array}{l}\Delta \mathrm{AIC} c \\
\text { (group) }\end{array}$ & $\begin{array}{c}\Delta \mathrm{AIC}_{c} \\
\text { (overall) }\end{array}$ & Deviance \\
\hline Photoperiod & 8 & $9,970.34$ & 130.31 & 132.04 & 128.822 \\
\hline Photoperiod + Discharge & 9 & $9,856.42$ & 16.39 & 18.12 & $9,838.398$ \\
\hline Photoperiod + Temperature & 9 & $9,883.91$ & 43.88 & 45.61 & $9,865.887$ \\
\hline Photoperiod + Weight & 9 & $9,972.22$ & 132.19 & 133.92 & $9,954.199$ \\
\hline Photoperiod $+\%$ Spill & 9 & $9,972.23$ & 132.21 & 133.93 & $9,954.213$ \\
\hline Photoperiod $+\%$ Spill + Discharge & 10 & $9,857.12$ & 17.09 & 18.81 & $9,837.090$ \\
\hline Photoperiod + Weight + Discharge & 10 & $9,856.97$ & 16.94 & 18.66 & $9,836.940$ \\
\hline Photoperiod + Weight + Temperature & 10 & $9,883.61$ & 43.58 & 45.31 & $9,863.582$ \\
\hline Photoperiod + Weight $+\%$ Spill & 10 & $9,974.16$ & 134.13 & 135.85 & $9,954.129$ \\
\hline Photoperiod $+\%$ Spill + Discharge + Temperature & 11 & $9,843.22$ & 3.19 & 4.91 & $9,821.186$ \\
\hline Photoperiod $+\%$ Spill + Discharge $+\%$ Spill $\times$ Discharge & 11 & $9,859.02$ & 18.99 & 20.72 & $9,836.991$ \\
\hline Photoperiod + Weight + Temperature + Discharge & 11 & $9,841.96$ & 1.94 & 3.66 & $9,819.932$ \\
\hline Photoperiod + Weight $+\%$ Spill + Discharge & 11 & $9,856.85$ & 16.83 & 18.55 & $9,834.822$ \\
\hline Photoperiod + Weight $+\%$ Spill + Discharge + Temperature & 12 & $9,840.03$ & 0.00 & 1.72 & $9,815.991$ \\
\hline Photoperiod $+\%$ Spill + Discharge + Temperature $+\%$ Spill $\times$ Discharge & 12 & $9,845.16$ & 5.13 & 6.86 & $9,821.122$ \\
\hline Photoperiod + Weight $+\%$ Spill + Discharge $+\%$ Spill $\times$ Discharge & 12 & $9,858.75$ & 18.73 & 20.45 & $9,834.717$ \\
\hline Photoperiod + Weight $+\%$ Spill + Discharge + Temperature $+\%$ Spill $\times$ Discharge & 13 & $9,841.97$ & 1.94 & 3.67 & $9,815.929$ \\
\hline
\end{tabular}


Table 30 (continued). Model selection for fish that passed through the spillway at McNary Dam relating survival (ø) as a function of environmental variables and group covariates for spillway passage location (i.e. north, south) using the fixed structure of the best model for $p$ and $\lambda$ parameters for subyearling Chinook salmon, 2004-09.

\begin{tabular}{|c|c|c|c|c|c|}
\hline Model & $\begin{array}{l}\text { Number of } \\
\text { parameters }\end{array}$ & $\mathrm{AIC} c$ & $\begin{array}{l}\Delta \mathrm{AIC} c \\
\text { (group) }\end{array}$ & $\begin{array}{c}\Delta \mathrm{AIC} c \\
\text { (overall) }\end{array}$ & Deviance \\
\hline SpillPassLoc & 9 & $9,975.29$ & 134.29 & 136.98 & 131.765 \\
\hline SpillPassLoc + Discharge & 10 & $9,857.40$ & 16.40 & 19.10 & $9,837.375$ \\
\hline SpillPassLoc + Temperature & 10 & $9,885.12$ & 44.12 & 46.82 & $9,865.096$ \\
\hline SpillPassLoc $+\%$ Spill & 10 & $9,977.28$ & 136.28 & 138.98 & $9,957.256$ \\
\hline SpillPassLoc + Weight & 10 & $9,977.27$ & 136.27 & 138.97 & $9,957.243$ \\
\hline SpillPassLoc + Temperature + Discharge & 11 & $9,843.78$ & 2.78 & 5.47 & $9,821.747$ \\
\hline SpillPassLoc $+\%$ Spill + Discharge & 11 & $9,858.48$ & 17.48 & 20.18 & $9,836.450$ \\
\hline SpillPassLoc + Temperature $+\%$ Spill & 11 & $9,885.68$ & 44.68 & 47.37 & $9,863.645$ \\
\hline SpillPassLoc + Weight + Temperature + Discharge & 12 & $9,841.72$ & 0.72 & 3.42 & $9,817.682$ \\
\hline SpillPassLoc + Temperature + Discharge $+\%$ Spill & 12 & $9,844.39$ & 3.39 & 6.09 & $9,820.353$ \\
\hline SpillPassLoc + Weight $+\%$ Spill + Discharge & 12 & $9,858.15$ & 17.15 & 19.85 & $9,834.116$ \\
\hline SpillPassLoc $+\%$ Spill + Discharge $+\%$ Spill $\times$ Discharge & 12 & $9,860.44$ & 19.44 & 22.13 & $9,836.400$ \\
\hline SpillPassLoc + Weight + Temperature + Discharge $+\%$ Spill & 13 & $9,841.00$ & 0.00 & 2.70 & $9,814.956$ \\
\hline SpillPassLoc + Temperature + Discharge $+\%$ Spill $+\%$ Spill $\times$ Discharge & 13 & $9,846.37$ & 5.37 & 8.07 & $9,820.330$ \\
\hline SpillPassLoc + Weight $+\%$ Spill + Discharge $+\%$ Spill $\times$ Discharge & 13 & $9,860.11$ & 19.11 & 21.81 & $9,834.065$ \\
\hline SpillPassLoc + Weight + Temperature + Discharge $+\%$ Spill $+\%$ Spill $\times$ Discharge & 14 & $9,842.99$ & 1.99 & 4.68 & $9,814.938$ \\
\hline
\end{tabular}


Table 30 (continued). Model selection for fish that passed through the spillway at McNary Dam relating survival ( $\varnothing)$ as a function of environmental variables and group covariates for spillway passage location (i.e. north, south) and photoperiod using the fixed structure of the best model for $p$ and $\lambda$ parameters for subyearling Chinook salmon, 2004-09.

\begin{tabular}{|c|c|c|c|c|c|}
\hline Model & $\begin{array}{l}\text { Number of } \\
\text { parameters }\end{array}$ & $\mathrm{AIC} c$ & $\begin{array}{c}\Delta \mathrm{AIC} c \\
\text { (group) }\end{array}$ & $\begin{array}{c}\Delta \mathrm{AIC} c \\
\text { (overall) }\end{array}$ & Deviance \\
\hline SpillPassLoc + Photoperiod & 10 & $9,967.76$ & 126.76 & 129.45 & 122.230 \\
\hline SpillPassLoc + Photoperiod + Discharge & 11 & $9,858.73$ & 16.04 & 20.43 & $9,836.701$ \\
\hline SpillPassLoc + Photoperiod + Temperature & 11 & $9,883.56$ & 40.86 & 45.25 & $9,861.524$ \\
\hline SpillPassLoc + Photoperiod $+\%$ Spill & 11 & $9,969.76$ & 127.07 & 131.46 & $9,947.730$ \\
\hline SpillPassLoc + Photoperiod + Weight & 11 & $9,969.72$ & 127.03 & 131.42 & $9,947.689$ \\
\hline SpillPassLoc + Photoperiod + Weight + Discharge & 12 & $9,859.03$ & 16.34 & 20.73 & $9,834.995$ \\
\hline SpillPassLoc + Photoperiod + Weight + Temperature & 12 & $9,882.43$ & 39.74 & 44.13 & $9,858.397$ \\
\hline SpillPassLoc + Photoperiod + Weight $+\%$ Spill & 12 & $9,971.73$ & 129.03 & 133.42 & $9,947.689$ \\
\hline SpillPassLoc + Photoperiod $+\%$ Spill + Discharge & 12 & $9,859.79$ & 17.10 & 21.49 & $9,835.752$ \\
\hline SpillPassLoc + Photoperiod $+\%$ Spill + Discharge + Temperature & 13 & $9,845.95$ & 3.25 & 7.64 & $9,819.902$ \\
\hline SpillPassLoc + Photoperiod + Weight $+\%$ Spill + Discharge & 13 & $9,859.55$ & 16.85 & 21.24 & $9,833.504$ \\
\hline SpillPassLoc + Photoperiod + Weight + Temperature + Discharge & 13 & $9,843.43$ & 0.74 & 5.13 & $9,817.389$ \\
\hline SpillPassLoc + Photoperiod $+\%$ Spill + Discharge $+\%$ Spill $\times$ Discharge & 13 & $9,861.76$ & 19.06 & 23.45 & $9,835.712$ \\
\hline SpillPassLoc + Photoperiod + Weight $+\%$ Spill + Discharge + Temperature & 14 & $9,842.69$ & 0.00 & 4.39 & $9,814.643$ \\
\hline SpillPassLoc + Photoperiod $+\%$ Spill + Discharge + Temperature $+\%$ Spill $\times$ Discharge & 14 & $9,847.93$ & 5.24 & 9.63 & $9,819.884$ \\
\hline SpillPassLoc + Photoperiod + Weight $+\%$ Spill + Discharge $+\%$ Spill $\times$ Discharge & 14 & $9,861.51$ & 18.82 & 23.21 & $9,833.461$ \\
\hline SpillPassLoc + Photoperiod + Weight $+\%$ Spill + Discharge + Temperature $+\%$ Spill $\times$ Discharge & 15 & $9,844.69$ & 1.99 & 6.38 & $9,814.628$ \\
\hline
\end{tabular}



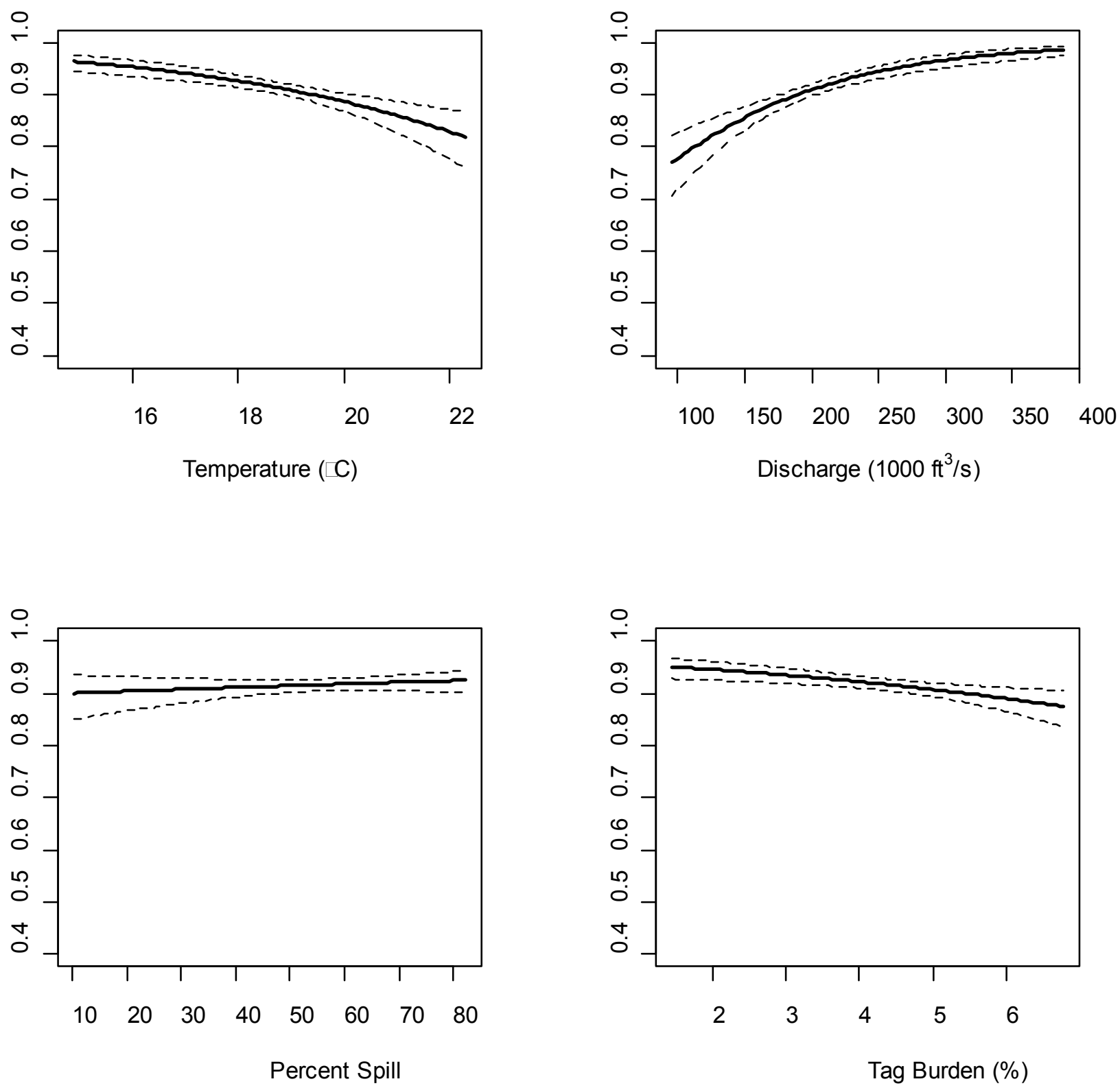

Figure 42. Survival of subyearling Chinook salmon passing through the spillway at McNary Dam in relation to water temperature, discharge, percent spill, and tag burden, 2004-09. The dashed lines represent the 95-percent confidence limits. Other covariates in the model were held constant at their mean value to examine the relationship to the variable of interest. 
Table 31. Model selection for fish released in the tailrace of McNary Dam relating survival ( $\varnothing)$ as a function of environmental variables using the fixed structure of the best model for $p$ and $\lambda$ parameters for subyearling Chinook salmon, 2004-09.

\begin{tabular}{lrrrrr}
\hline \multicolumn{1}{c}{ Model } & $\begin{array}{c}\text { Number of } \\
\text { parameters }\end{array}$ & AIC $\boldsymbol{c}$ & $\begin{array}{c}\Delta \text { AIC } \boldsymbol{c} \\
\text { (group) }\end{array}$ & $\begin{array}{c}\Delta \text { AIC } \boldsymbol{c} \\
\text { (overall) }\end{array}$ & \multicolumn{1}{c}{ Deviance } \\
\hline Intercept only & 13 & $13,469.56$ & 110.35 & 112.96 & 73.204 \\
Temperature & 14 & $13,359.42$ & 0.21 & 2.82 & $13,331.381$ \\
Discharge & 14 & $13,404.91$ & 45.70 & 48.31 & $13,376.871$ \\
\%Spill & 14 & $13,463.39$ & 104.18 & 106.80 & $13,435.356$ \\
Weight & 14 & $13,467.50$ & 108.29 & 110.90 & $13,439.461$ \\
Temperature + Discharge & 15 & $13,359.21$ & 0.00 & 2.61 & $13,329.166$ \\
Temperature + \%Spill & 15 & $13,359.43$ & 0.22 & 2.83 & $13,329.383$ \\
\%Spill + Discharge & 15 & $13,406.17$ & 46.96 & 49.57 & $13,376.126$ \\
Temperature + Discharge + \%Spill & 16 & $13,360.16$ & 0.95 & 3.56 & $13,328.107$ \\
Weight + Temperature + Discharge & 16 & $13,360.73$ & 1.52 & 4.13 & $13,328.678$ \\
Weight + Temperature + \%Spill & 16 & $13,360.77$ & 1.56 & 4.18 & $13,328.725$ \\
\%Spill + Discharge + \%Spill $\times$ Discharge & 16 & $13,407.61$ & 48.40 & 51.01 & $13,375.562$ \\
Weight + \%Spill + Discharge & 16 & $13,408.17$ & 48.97 & 51.58 & $13,376.126$ \\
Weight + Temperature + Discharge + \%Spill & 17 & $13,361.36$ & 2.15 & 4.76 & $13,327.303$ \\
Temperature + Discharge + \%Spill + \%Spill $\times$ Discharge & 17 & $13,362.11$ & 2.90 & 5.51 & $13,328.057$ \\
Weight + \%Spill + Discharge + \%Spill $\times$ Discharge & 17 & $13,409.61$ & 50.41 & 53.02 & $13,375.560$ \\
Weight + Temperature + Discharge + \%Spill + \%Spill $\times$ Discharge & 18 & $13,363.36$ & 4.15 & 6.76 & $13,327.294$ \\
\hline
\end{tabular}


Table 31 (continued). Model selection for fish released in the tailrace of McNary Dam relating survival $(\varnothing)$ as a function of environmental variables and group covariates for photoperiod using the fixed structure of the best model for $p$ and $\lambda$ parameters for subyearling Chinook salmon, 2004-09.

[The best-fit model is indicated in bold]

\begin{tabular}{lrrrrr}
\hline \multicolumn{1}{c}{ Model } & $\begin{array}{c}\text { Number of } \\
\text { parameters }\end{array}$ & AIC $\boldsymbol{c}$ & $\begin{array}{c}\Delta \text { AIC } \boldsymbol{c} \\
\text { (group) }\end{array}$ & \multicolumn{1}{c}{$\begin{array}{c}\Delta \text { AIC } \boldsymbol{c} \\
\text { (overall) }\end{array}$} & \multicolumn{1}{c}{ Deviance } \\
\hline Photoperiod & 14 & $13,461.24$ & 104.64 & 104.64 & 62.881 \\
Photoperiod + Temperature & $\mathbf{1 5}$ & $\mathbf{1 3 , 3 5 6 . 6 0}$ & $\mathbf{0 . 0 0}$ & $\mathbf{0 . 0 0}$ & $\mathbf{1 3 , 3 2 6 . 5 5 5}$ \\
Photoperiod + \%Spill & 15 & $13,453.47$ & 96.87 & 96.87 & $13,423.423$ \\
Photoperiod + Discharge & 15 & $13,404.69$ & 48.09 & 48.09 & $13,374.646$ \\
Photoperiod + Weight & 15 & $13,458.92$ & 102.33 & 102.33 & $13,428.881$ \\
Photoperiod + Temperature + Weight & 16 & $13,358.44$ & 1.84 & 1.84 & $13,326.387$ \\
Photoperiod + \%Spill + Discharge & 16 & $13,405.61$ & 49.01 & 49.01 & $13,373.558$ \\
Photoperiod + Weight + Discharge & 16 & $13,406.66$ & 50.06 & 50.06 & $13,374.611$ \\
Photoperiod + Weight + \%Spill & 16 & $13,453.35$ & 96.75 & 96.75 & $13,421.302$ \\
Photoperiod + \%Spill + Discharge + Temperature & 17 & $13,357.86$ & 1.26 & 1.26 & $13,323.805$ \\
Photoperiod + \%Spill + Discharge + \%Spill $\times$ Discharge & 17 & $13,407.11$ & 50.52 & 50.52 & $13,373.060$ \\
Photoperiod + Weight + \%Spill + Discharge & 17 & $13,407.61$ & 51.01 & 51.01 & $13,373.558$ \\
Photoperiod + Weight + \%Spill + Discharge + Temperature & 18 & $13,359.19$ & 2.59 & 2.59 & $13,323.130$ \\
Photoperiod + \%Spill + Discharge + Temperature + \%Spill $\times$ Discharge & 18 & $13,359.85$ & 3.25 & 3.25 & $13,323.788$ \\
Photoperiod + Weight + \%Spill + Discharge + \%Spill $\times$ Discharge & 18 & $13,409.12$ & 52.52 & 52.52 & $13,373.055$ \\
Photoperiod + Weight + \%Spill + Discharge + Temperature + \%Spill $\times$ Discharge & 19 & $13,361.20$ & 4.60 & 4.60 & $13,323.130$ \\
\hline
\end{tabular}




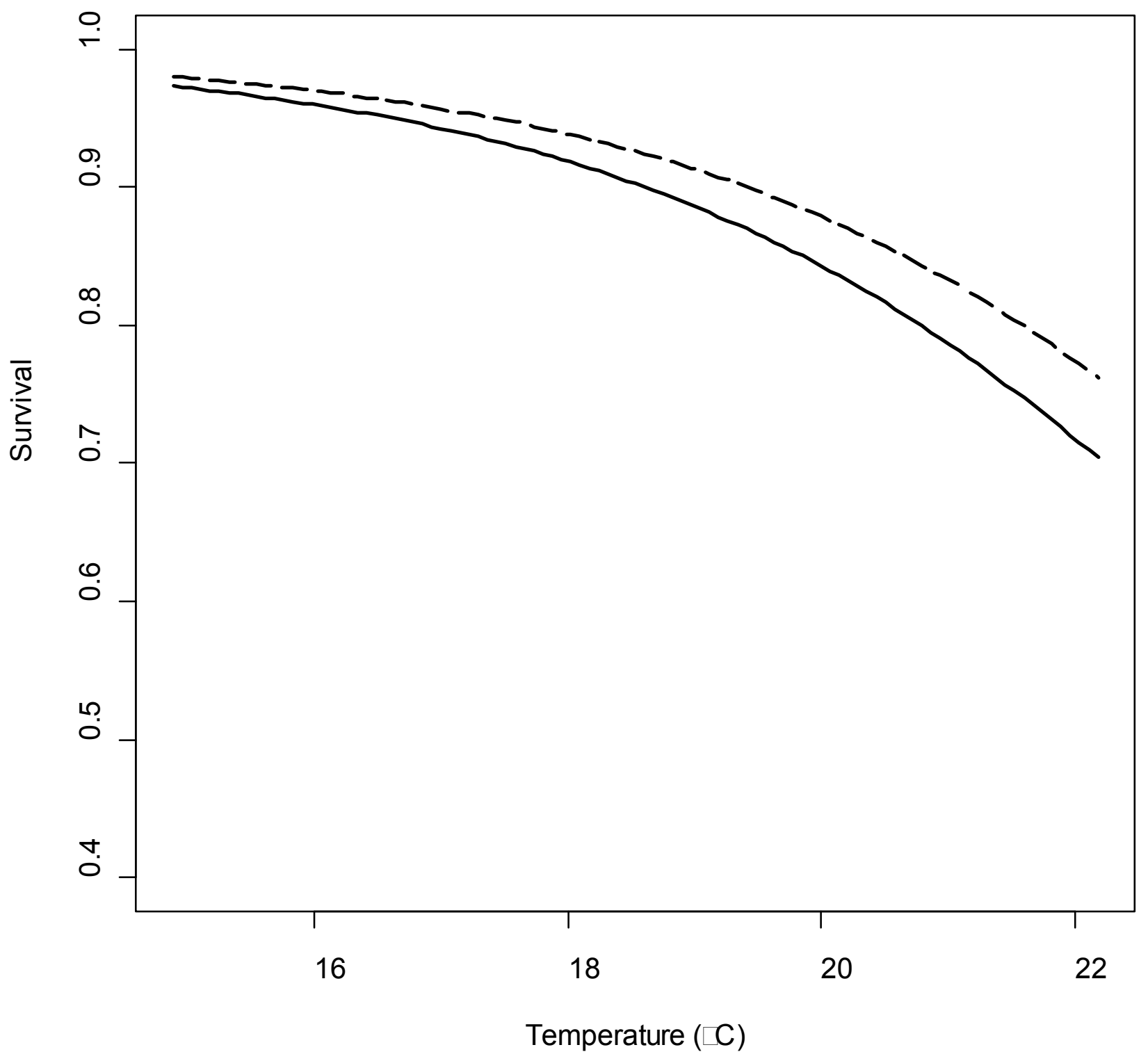

Figure 43. Survival of subyearling Chinook salmon released in the tailrace of McNary Dam in relation to water temperature during day (dashed line) and night (solid line) photoperiods, 2004-09. 


\section{Temporal Survival Estimates}

Survival estimates at the weekly (table A3) and annual (table 32) temporal scale were generated to provide temporal information for survival through routes and to compare estimates predicted by covariates. Survival through the forebay on a weekly basis varied between 0.90 and 1.00, depending on the year of study, but less variation was observed from week to week within each year of study (fig. 44). For all passage routes, survival decreased as the summer migration season progressed (fig. 45). This decrease in survival was higher for juvenile salmon passing through the juvenile bypass system and turbine passage routes. Survival was lowest during the last week in 2009 for all passage routes, although this was not observed for fish released in the tailrace during this time. Estimates of forebay survival were better predicted on an annual scale compared to weekly (fig. 46). Covariates predicted weekly survival estimates the best for fish passing through the spillway and for fish released in the tailrace of McNary Dam (fig. 47). At the annual temporal scale, covariates best predicted survival for fish passing through the spillway and turbine routes and for fish released in the tailrace (fig. 48).

Table 32. Annual survival estimates during the summer migration period for subyearling Chinook salmon passing through and released in the tailrace of McNary Dam, 2004-09.

[Standard error is in parentheses]

\begin{tabular}{lcccccc}
\hline & $\mathbf{2 0 0 4}$ & $\mathbf{2 0 0 5}$ & $\mathbf{2 0 0 6}$ & $\mathbf{2 0 0 7}$ & 2008 & 2009 \\
\hline Forebay & $\mathrm{NA}$ & $0.937(0.009)$ & $0.999(0.003)$ & $0.987(0.004)$ & $0.996(0.003)$ & $0.973(0.005)$ \\
Turbine & $0.680(0.025)$ & $0.764(0.047)$ & $0.741(0.023)$ & $0.728(0.035)$ & $0.770(0.025)$ & $0.670(0.028)$ \\
Bypass & $0.736(0.027)$ & $0.822(0.035)$ & $0.899(0.018)$ & $0.897(0.034)$ & $0.844(0.025)$ & $0.853(0.022)$ \\
Spillway & $\mathrm{NA}$ & $0.899(0.012)$ & $0.945(0.010)$ & $0.880(0.017)$ & $0.913(0.009)$ & $0.855(0.011)$ \\
Tailrace/Control & $0.844(0.016)$ & $0.896(0.013)$ & $0.933(0.009)$ & $0.925(0.019)$ & $0.909(0.009)$ & $0.908(0.008)$ \\
\hline
\end{tabular}




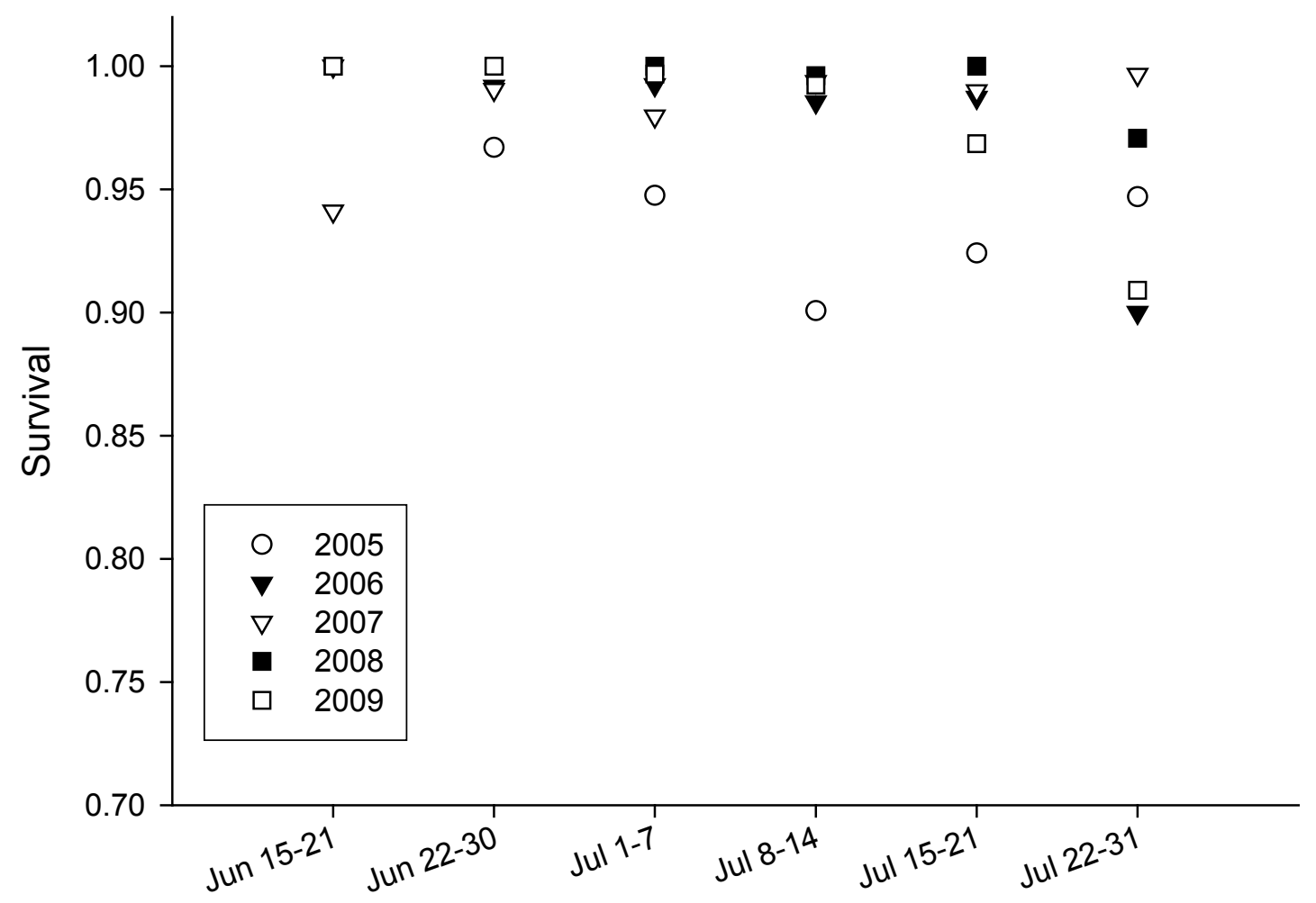

Figure 44. Weekly survival of subyearling Chinook salmon in the forebay of McNary Dam, 2004-09. 
Turbine

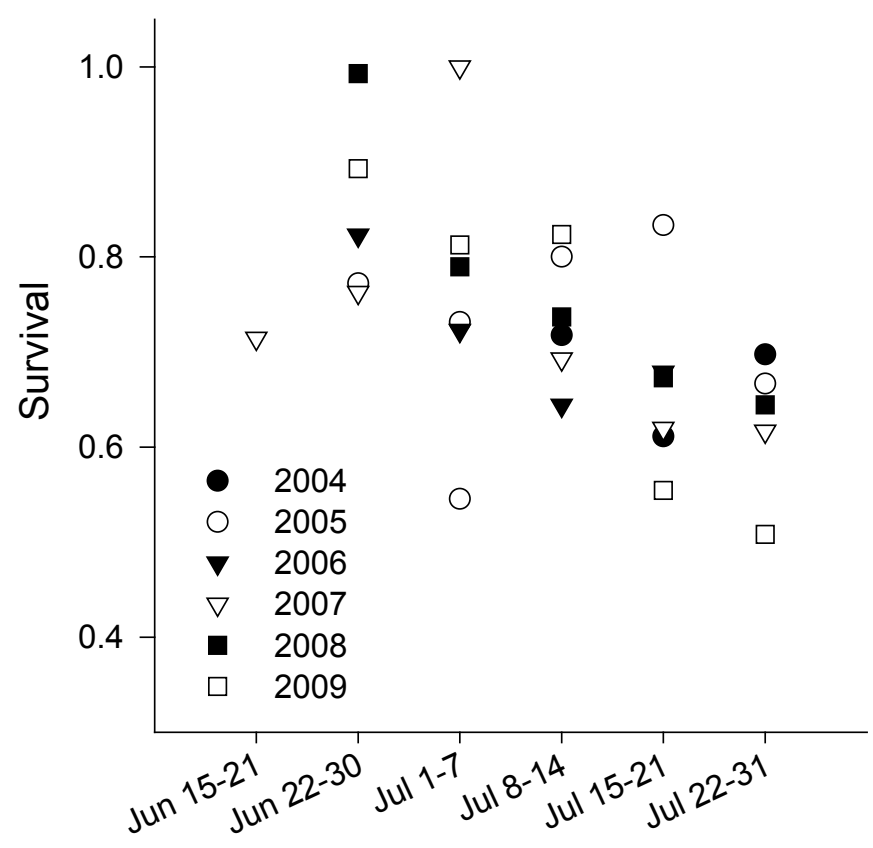

Spillway

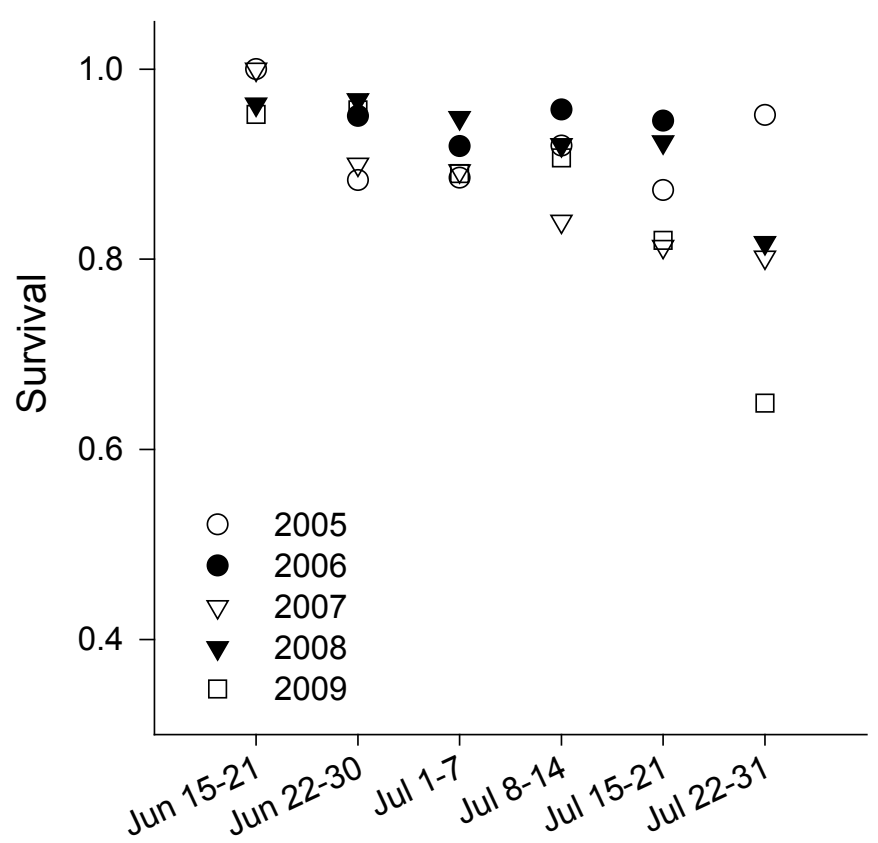

Bypass

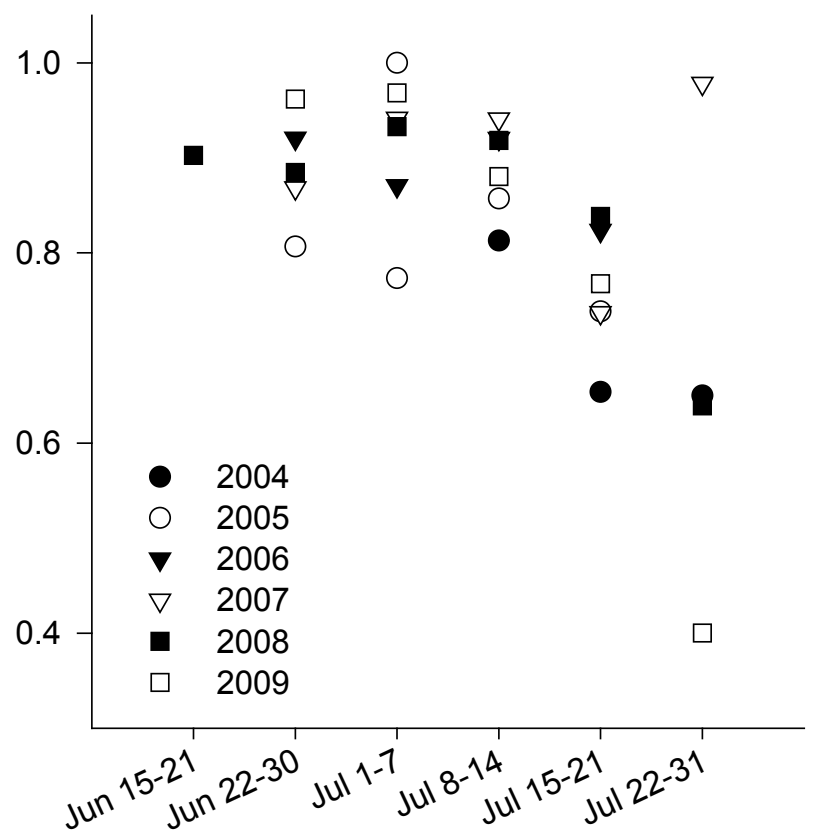

Control/Tailrace

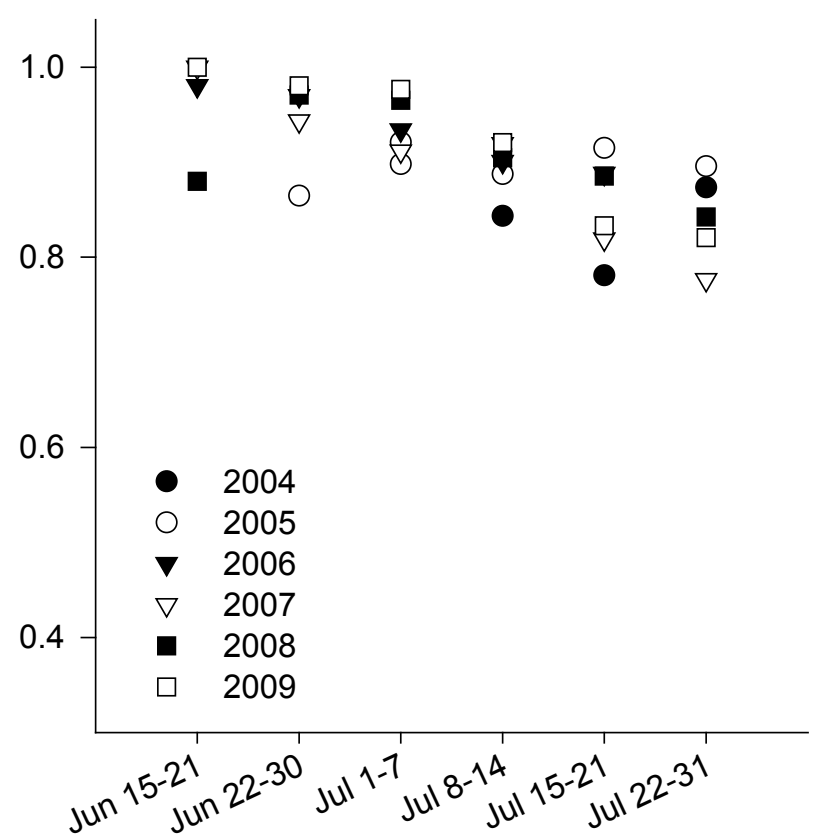

Figure 45. Weekly survival of subyearling Chinook salmon for fish that passed through the juvenile bypass system, spillway, turbines, or that were released in the tailrace of McNary Dam, 2004-09. 

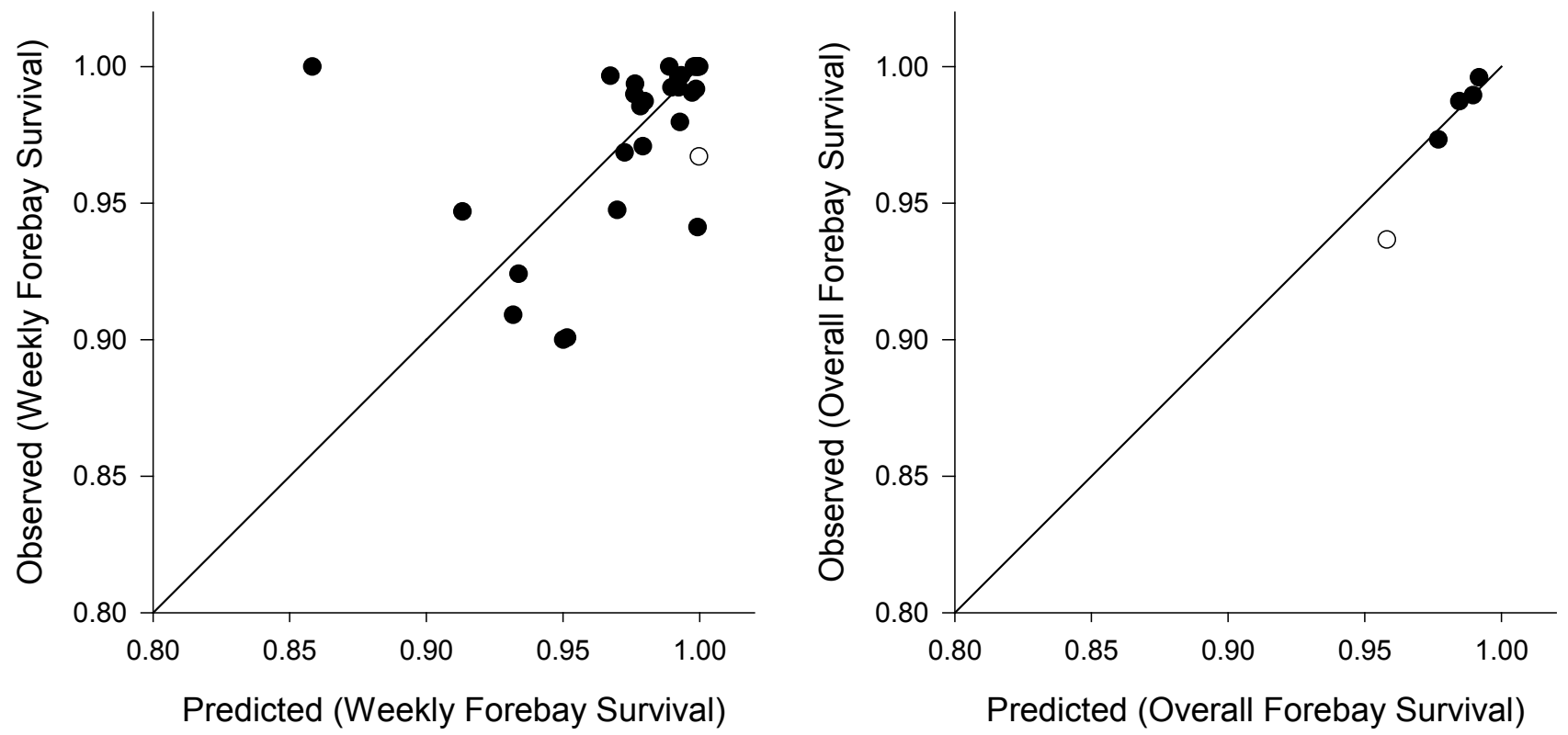

Figure 46. Observed versus predicted weekly (left) and annual survival (right) of subyearling Chinook salmon during the summer migration period in the forebay of McNary Dam, 2005-09. Solid line indicates graph coordinates where observed and predicted survival are equivalent. 
Turbine

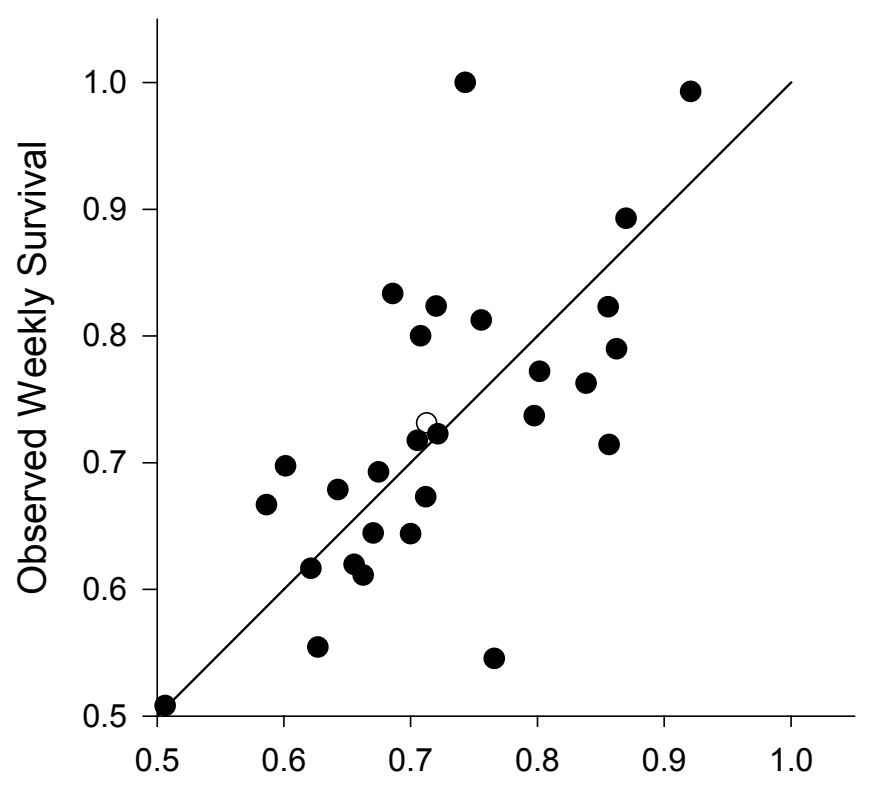

Spllway

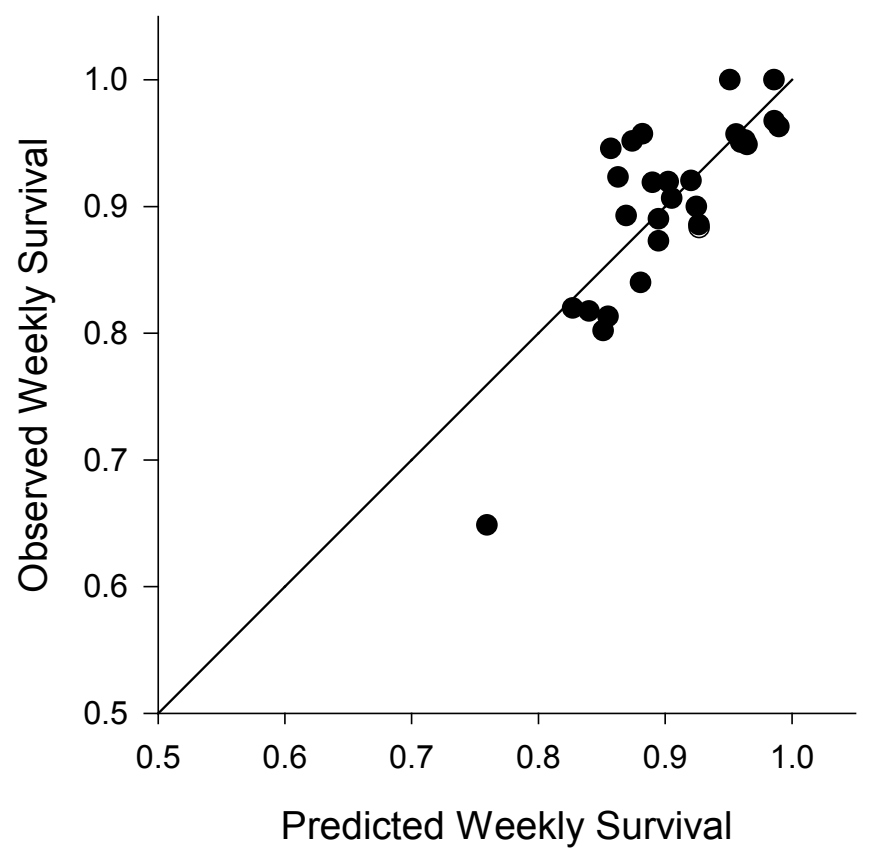

Bypass

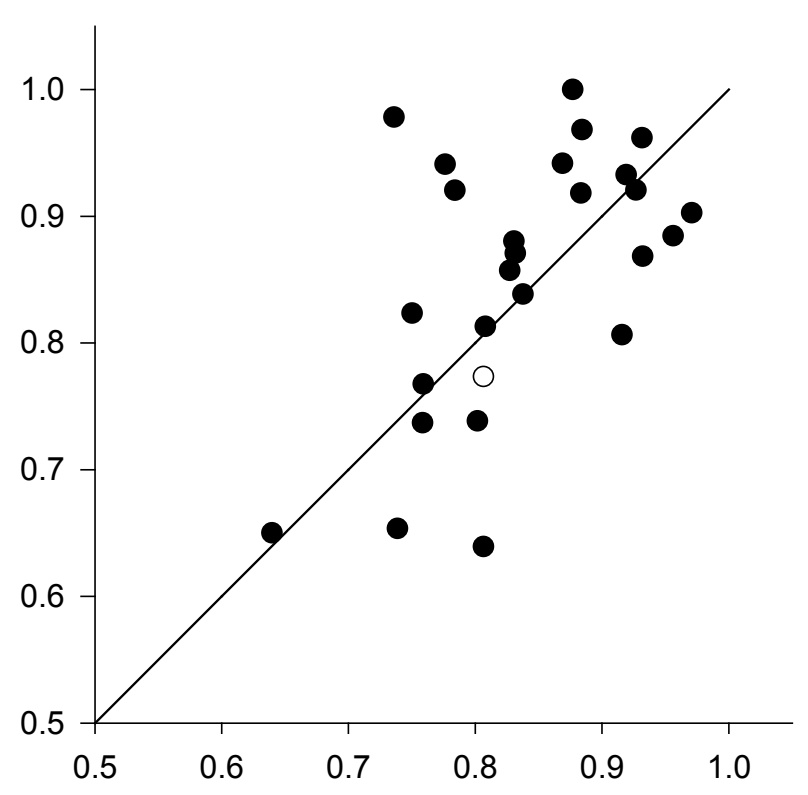

Tailrace/Control

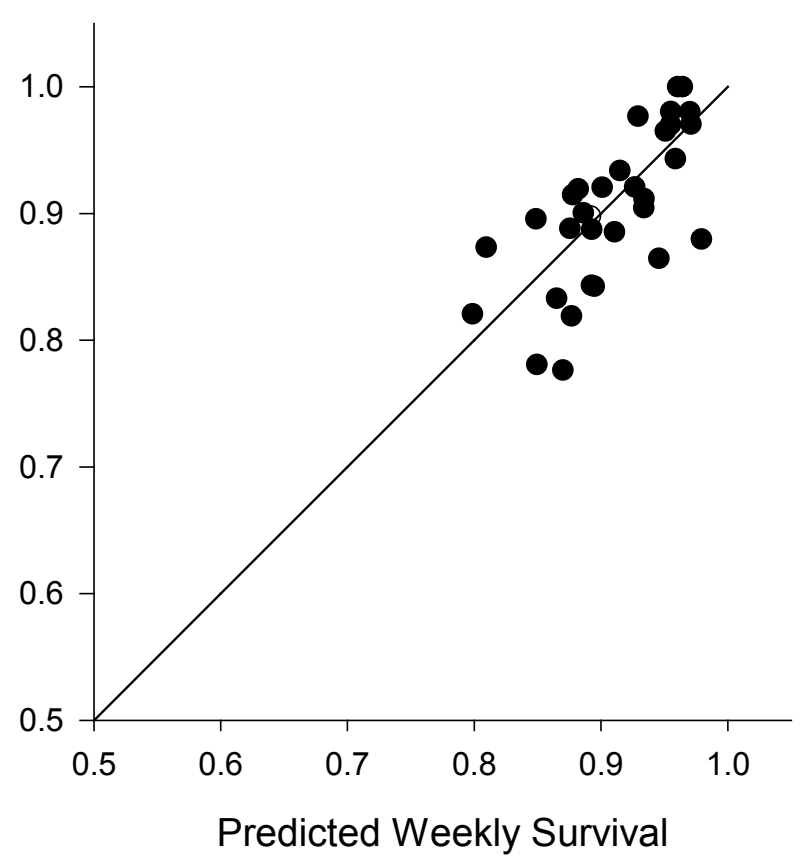

Figure 47. Observed versus predicted weekly survival of subyearling Chinook salmon for fish that passed through the juvenile bypass system, spillway, turbines, or that were released in the tailrace of McNary Dam, 2004-09. Solid line indicates graph coordinates where observed and predicted survival are equivalent. 
Turbine

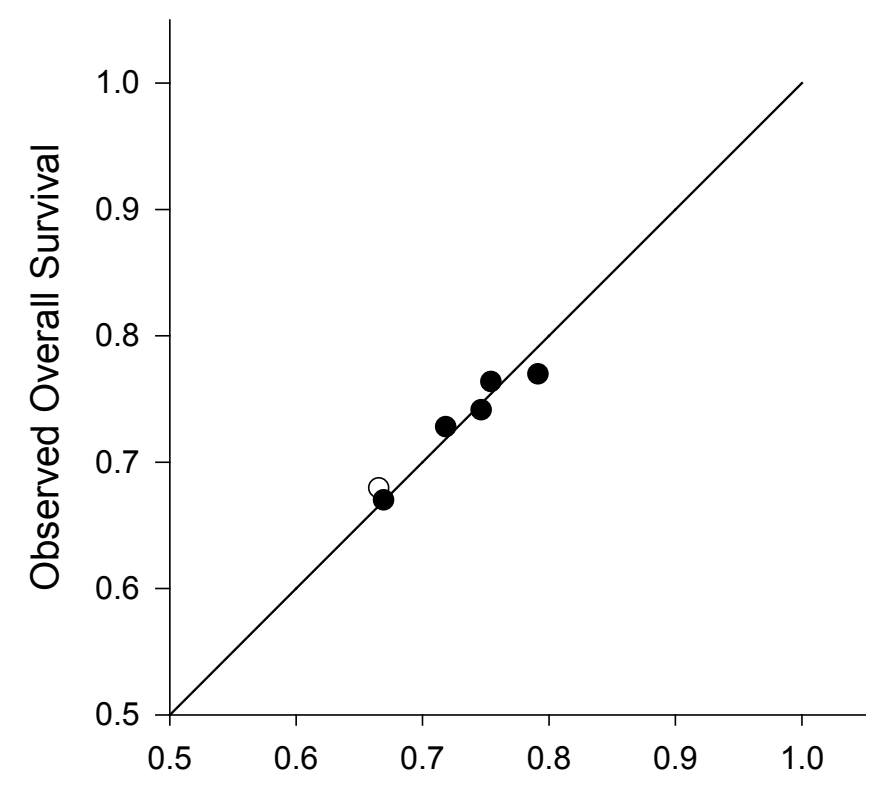

Spillway

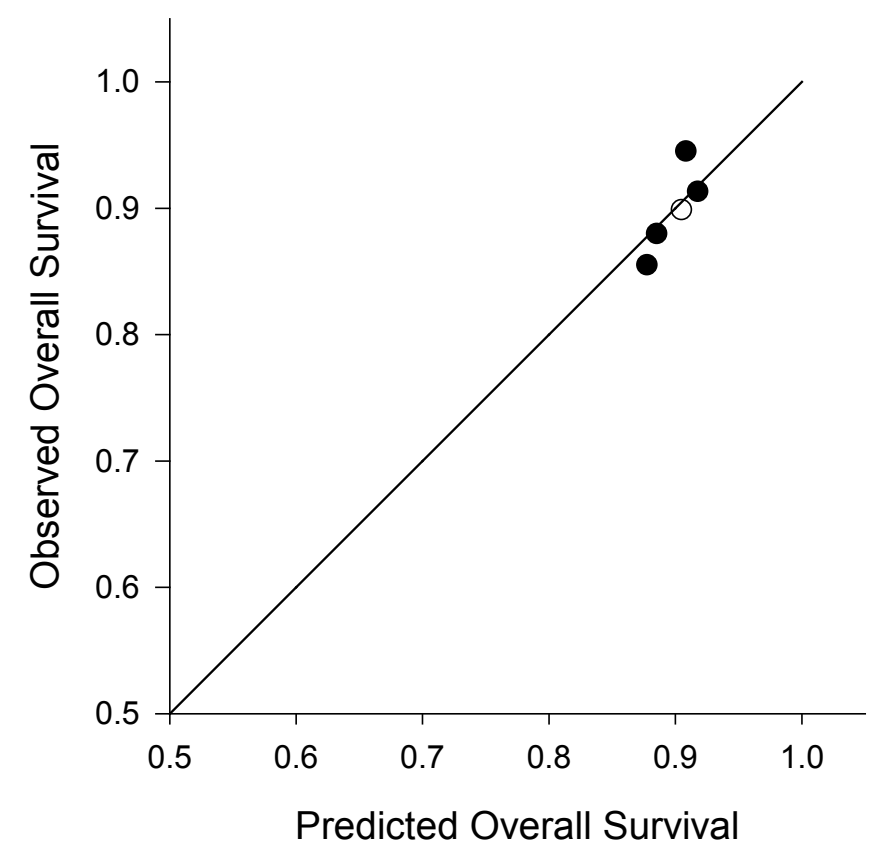

Bypass

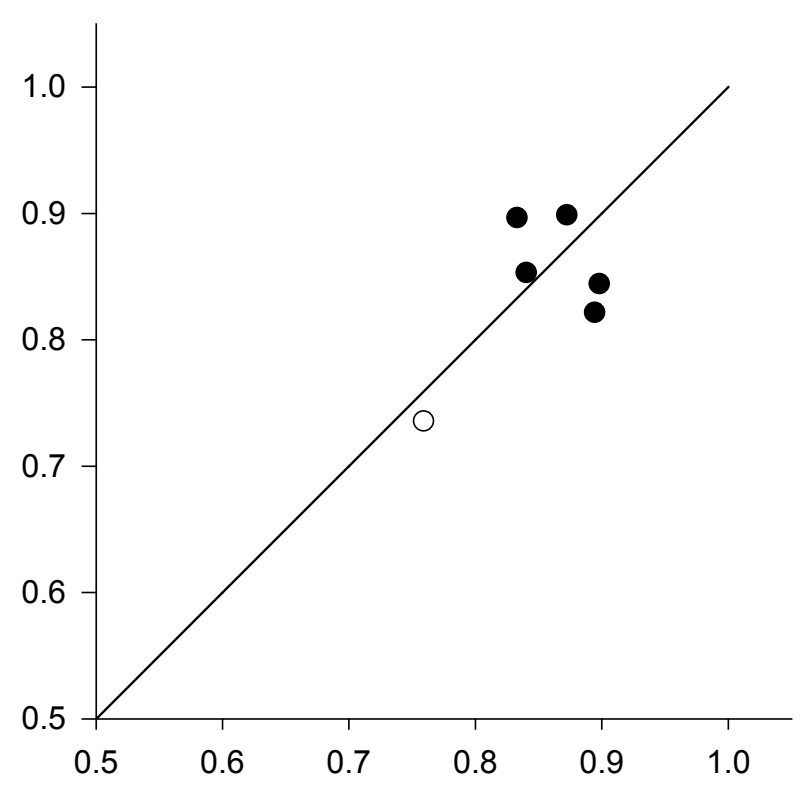

Tailrace/Control

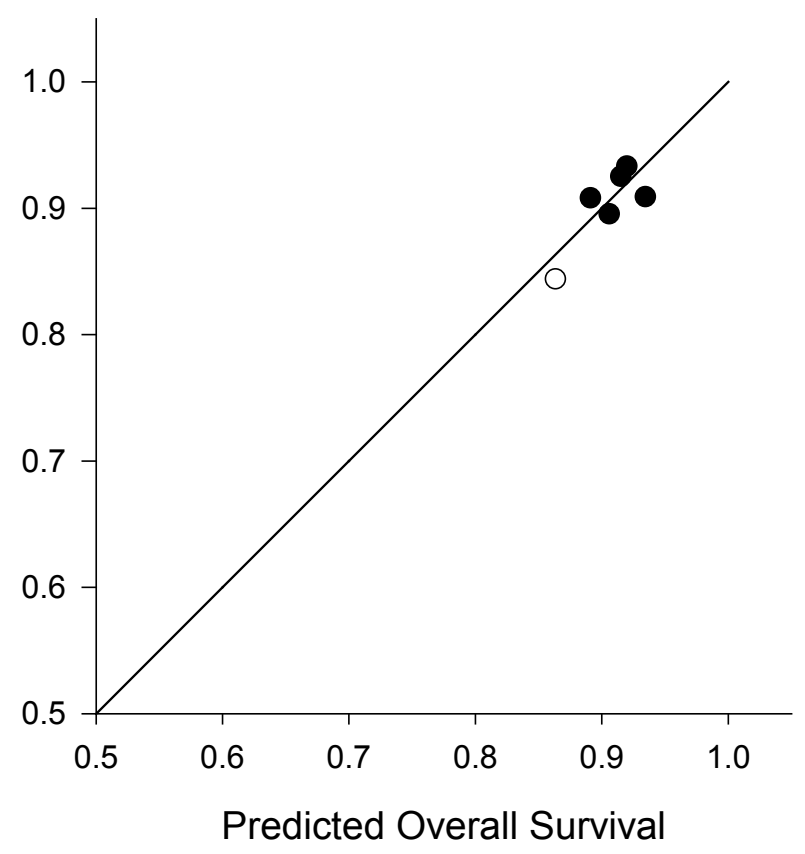

Figure 48. Observed versus predicted annual survival of subyearling Chinook salmon during the summer migration period for fish that passed through the juvenile bypass system, spillway, turbines, or that were released in the tailrace of McNary Dam, 2004-09. Solid line indicates graph coordinates where observed and predicted survival are equivalent. 


\section{Discussion}

\section{Passage}

Juvenile salmonid passage at McNary Dam was affected by dam operations, environmental conditions (for example, river discharge), and individual traits (for example, fish size). The extent to which these factors influenced passage varied by species and passage route. For yearling Chinook salmon and steelhead, total discharge and percent spill were important variables explaining passage probabilities at McNary Dam. When percent spill increased, passage through the spillway increased and passage through the turbines and juvenile bypass system decreased. However, the effect of spill on passage through the spillway and bypass system depended on the amount of total discharge passing the dam. For example, at 60 percent spill, spillway passage increased as total discharge increased, but bypass passage decreased as total discharge increased. In contrast, at 20 percent spill, both spillway and bypass passage increased with total discharge. These relationships interacted to influence turbine passage. At high percent spill, turbine passage was relatively constant, but at low percent spill, turbine passage decreased with increasing total discharge. These relationships were likely influenced by changes in flow patterns upstream of the dam as influenced by total discharge. When total discharge was relatively low and turbine discharge was high ( 80 percent), the movement of water into the turbine intake may have occurred relatively close to the face of the dam. As fish traveled towards the face of the dam, they were likely relatively shallow.

The vertical distribution of fish upstream of hydroelectric dams has been reported extensively in the literature (Adams and others, 2008). Once at the face of the dam, fish could have moved deeper in search of a passage route and likely detected flow vectors moving toward the turbine intake. As they continued moving deeper in the water, parallel to the vertical face of the dam, they would have sensed the flow going into the turbine intakes and subsequently passed through the dam. The flow characteristic inside the turbine intakes when total discharge is relatively low would have influenced whether fish passed through the turbines or been diverted by the screens into the bypass facility. When total discharge was high, and turbine discharge remained high ( 80 percent), the movement of water into the turbine intakes may have occurred farther upstream from the face of the dam. This would have resulted in water moving into the turbine intakes at more of a perpendicular angle to the vertical face of the dam. Therefore, the stream lines entering the turbine intake would have been at a flatter angle when total discharge was high. The change in the angle at which water approached the powerhouse has been observed in computational fluid dynamic models upstream of turbine intakes for several hydroelectric dams on the Columbia River. If this were the case, fish traveling towards the face of the dam would likely detect water moving into the turbine intakes farther upstream of the dam and could have moved deeper in the water column. Once fish reached the face of the dam, they would have been moving perpendicular to the vertical face of the dam, at a relatively greater depth in the water column, and some fish could have been diverted by the screens into the bypass system, thereby increasing the proportion of fish passing through that route. These mechanistic processes are plausible explanation for the patterns in passage that we quantified. However, these are not the only mechanisms that could have influenced fish passage through the powerhouse. 
The percentage of water spilled also was important in explaining passage probabilities for subyearling Chinook salmon passing McNary Dam. Similar to spring migrants, spillway passage was positively related to spill percent, while turbine and bypass passage was negatively related to spill. These results indicated that higher percent spill levels (60 percent) over the range of total discharge is one way to pass more fish through the spillway and less through the turbines and juvenile bypass system. In contrast to spring migrants, passage probabilities for subyearling Chinook salmon through the turbines and bypass were less sensitive to total discharge. It is possible that maximum total discharge during the summer was too low to flatten the streamlines entering the turbine intakes and affect the proportion of fish diverted by the screens into the bypass system. Alternatively, because subyearling Chinook salmon tend to travel deeper in the water column than spring migrants, the effect that total discharge had on streamlines moving into the turbines may have had less influence on turbine passage of subyearling Chinook salmon.

Water temperature also influenced passage of subyearling Chinook salmon. As water temperature increased, fish passage decreased through the spillway and juvenile bypass system and increased through turbines. Increased turbine passage during periods of increased water temperatures was observed in the latter portion of the summer migration season during 2009 (Adams and Liedtke, 2010). Water temperatures in the McNary Dam forebay are known to vertically stratify in the summer during low-wind periods. Therefore, subyearling Chinook salmon might have traveled deeper in the water in response to increased water temperatures near the surface. Fish traveling deeper in the water column might have entered the turbine intake at a depth below the tip of the guidance screens. This would have resulted in a decrease in the number of fish diverted by the screens into the bypass system and an increase in the number of fish passing into the turbines.

The presence of a TSW, the year the study was conducted, and photoperiod also were factors that were related to changes in passage probabilities. For yearling Chinook salmon, subyearling Chinook salmon, and steelhead, the TSW factor (which indicated years when TSWs were installed) was significant in explaining variability in passage probabilities, but only when the year factor was not included in the model. The effect of the TSW on passage probabilities appeared to indicate that the TSWs may have caused fish to move toward the spillway that would have otherwise passed through the powerhouse. However, when the year factor was included in the models, the TSW factor was no longer significant because the year factor fit the probabilities in the model better. The year factor likely explained more of the variability in the model because it includes both the variability explained by the TSW factor as well as any additional variation in environmental conditions from year to year. Therefore, although we suspect that the TSW may have increase spillway passage probabilities, we cannot rule out other potential mechanisms that differed between years with and without TSWs. To better understand the effect of the TSW on passage probabilities, a study would need to be conducted based on a randomized block design that includes periods of time with and without the TSWs installed during a single season.

Photoperiod was significant in explaining passage probabilities but was highly variable across a range of environmental conditions such as discharge and percent spill. Model results for all species indicated higher passage through the spillway during the day. The presence of the TSWs might have provided a surface oriented passage route that resulted in increased day-time passage through the spillway. An additional post hoc assessment showed that there was a significant effect of the TSW on daytime passage through the spillway, particularly for steelhead. 
Model results were inconclusive and variable regarding the influence of fish weight and tag burden on passage probabilities. However, it is possible that the weight of the fish, irrespective of the presence of the tag, might have had an influence on fish passage. As fish grow larger and begin the smoltification process, they become more buoyant (Wedemeyer and others, 1980). Larger fish that are more buoyant may travel higher in the water column and be less likely to enter the turbine intakes and juvenile bypass system. This may explain why passage probabilities in the spillway increased as fish weight increased for subyearling Chinook salmon and steelhead. The increased buoyancy of these fish might have caused them to travel higher in the water column while searching for a passage route. As they traversed the face of the dam in search of a passage route, they would have encountered the surface oriented passage route (TSW) in the spillway.

\section{Survival}

Factors that influenced passage probabilities also influenced survival. Predominant variables that influenced survival for all passage routes were percent spill, total discharge, and temperature. In many instances, survival was positively correlated with increases in discharge and percent spill, and inversely related to increasing temperatures, particularly for subyearling Chinook salmon. For some analyses, survival was positively correlated with fish weight. Because fish weight is inversely related to tag burden, it was not surprising to also observe instances when survival increased as tag burden decreased. Due to the inherent correlation between fish weight and tag burden, the effect of these two factors on survival could not be isolated. Caution should be used when interpreting model results that indicate one of these two factors had more of an influence on survival. For example, fish weight was a significant factor in the model for yearling Chinook salmon survival through the forebay. However, it could not be determined whether fish weight or tag burden had more of an effect on the survival of yearling Chinook salmon passing through the turbines and the spillway. Similar variability existed for subyearling Chinook salmon.

Operation of the TSWs appeared to increase forebay survival for both yearling Chinook salmon and steelhead, especially when total discharge was less than $300 \mathrm{kcfs}$. This could indicate that when flows were less than $300 \mathrm{kcfs}$, the flow entering the TSW was more predominant relative to the overall flow in the forebay and more fish passed through the TSW route. When discharge was greater than 300 $\mathrm{kcfs}$, the flow into the TSW would have been diminished relative to the overall flow passing through the dam and the effect of the TSW on forebay survival also would have been diminished. However, higher flows through all routes when discharge was greater than $300 \mathrm{kcfs}$ might have resulted in more fish passing in a shorter amount of time and could have led to an increase in forebay survival despite the diminished effect of the TSW during higher flows.

The TSW had the greatest effect on forebay survival of steelhead. Given that steelhead tend to migrate nearer to the surface than juvenile Chinook salmon do, it is not surprising that a surface oriented passage route like the TSW would have had a positive effect on forebay survival. During years when the TSW was not installed, forebay survival for steelhead was lowest when fish arrived in the forebay during day time periods with low discharge. When discharge was low, steelhead may have spent more time searching for a passage route, especially during the day, and could have been exposed to increased predation in the forebay. At night, predation rates would naturally be lower because visually oriented predators in the forebay would be less successful. In addition, steelheads tend to move deeper in the water column at night and could have more readily detected the flow passing through the spillway. As 
discharge increases, the flow passing through the spillway increases, making it easier for the steelhead to detect and pass the dam, even during the day when they are relatively shallow. During years when the TSW was installed, forebay survival generally was higher, and survival was nearly equal during the day and night across the entire range of discharge. The surface oriented passage route provided by the TSW could have allowed steelhead to find a passage route relatively quickly, especially during the day when they tend to be higher in the water column. In contrast, the TSW factor was not significant in explaining survival through the forebay for subyearling Chinook salmon. Because subyearling Chinook salmon tend to migrate deeper in the water column, the effect of the TSW on forebay survival may not have been as great.

\section{Passage and Survival}

The results of these analyses, while they were not designed to yield predictive models, can be used to help develop dam-operation strategies to optimize survival. For example, total discharge and the proportion of flow allocated to the spillway typically had a positive effect on survival for all species and routes. As expected, increases in the proportion of water going through the spillway resulted in increased spillway passage. The strong linear relation between increased spill and increased survival indicates that increasing the proportion of water through the spillway is one strategy that could be used to improve survival for all species. Furthermore, results showed that survival generally was higher at night, so increased spill at night might result in further increases in survival. Flow distribution across the spillway did not appear to affect spillway survival. We found no correlation between survival and whether fish passed through the north or south portion of the spillway. The same was true for turbine survival: there was no difference in survival for fish passing through the north or south portion of the powerhouse. Further analysis is being conducted to investigate how spillway operations could influence dam survival and the results will be presented in a separate report. However, results from the current analysis indicate that conditions created in the tailrace as a result of the spill pattern may have affected survival.

The survival of subyearling Chinook salmon passing through the turbines was correlated to percent spill. When discharge was less than about $175 \mathrm{kcfs}$, turbine survival was inversely related to percent spill and when discharge was greater than $175 \mathrm{kcfs}$, turbine survival was positively correlated to percent spill. These relationships were likely influenced by tailrace conditions that favored increased predation of fish passing through the turbines. When discharge was less than $175 \mathrm{kcfs}$, the relatively high volume of flow through the spillway (60 percent) could have forced the relatively low volume of water leaving the powerhouse to flow along the south shore of the tailrace where predatory birds and fish were prevalent. When less water was spilled (20 percent) and more water passed through the powerhouse ( 80 percent), water flowing out of the powerhouse would have shifted north, away from the predators along the south shore. When discharge was greater than $175 \mathrm{kcfs}$, high volumes of spill (60 percent), together with relatively higher bulk flow leaving the powerhouse, could have combined to create favorable tailrace conditions for fish leaving the turbines. Under these conditions, fish leaving the powerhouse tailrace would egress quickly and closer to the center of the river, away from shoreline habitat where predators reside. When discharge was high and most of the water passed through the powerhouse ( 80 percent), more of the fish exiting the turbines could have been exposed to the predators along the south shore. However, exposure time was likely relatively short due to increased velocity in the tailrace when discharge was high. 
Dam-operation strategies also should account for the effect that increased water temperature had on the survival of subyearling Chinook salmon. Results showed that increased temperatures had a dramatic effect on passage and resulted in a decrease in survival through most routes. Increasing the amount of water passing the spillway, especially when water temperatures are high later in the summer, is one way to increase the survival for subyearling Chinook salmon. Because there appeared to be higher survival during the night, increased spill at night might result in further increases in survival. Increasing spill may be an effective way to improve survival in the warmer summer months, but consideration also must be given to the potential effect that elevated total dissolved gas levels in the tailrace might have on fish survival.

Although the results were somewhat inconclusive due to annual variation, the presence of a surface oriented passage route (like the TSW) could be an important part of the operational strategy to improve project survival. This may be especially true when total project discharge is less than about 300 kcfs and water temperatures are elevated. Forebay survival appeared to have improved during years when the TSWs were installed and the number of fish passing through the spillway, a route of passage with relatively high survival, likely increased due to the presence of the TSW.

This analysis provided a unique opportunity to examine data from multiple years of research to investigate factors influencing passage and survival at McNary Dam. By first using statistical models to select among variables hypothesized to influence passage and survival, and then quantifying the magnitude of the effects, we were able to examine how changes to dam operations might positively or negatively influence survival. This is critical information that managers need to develop long-term operational plans. Although development of predictive models was beyond the scope of our analysis, the relations we identified could be used to develop simulation models to gauge the effect of management actions on dam survival.

\section{References Cited}

Adams, N.S., and Evans, S.D., eds., 2011, Summary of juvenile salmonid passage and survival at McNary Dam: Acoustic telemetry studies, Report Series 2011-1179, U. S. Geological Survey.

Adams, N.S., and Counihan, T.D., eds, 2009, Survival and migration behavior of juvenile salmonids at McNary Dam, 2007: U. S. Geological Survey, Report to the U. S. Army Corps of Engineers, Walla Walla District, Contract no. W68SBV70178419.

Adams, N.S., and Liedtke, T.L., eds., 2010, Juvenile salmonid survival, passage, and egress at McNary Dam during tests of temporary spillway weirs, 2009: U. S. Geological Survey, Report to the U. S. Army Corps of Engineers, Contract no. W68SBV90070150.

Adams, N.S., Plumb, J.M., Hatton, T.W., Jones, E.C., Swyers, N.M., Sholtis, M.D., Reagan, R.E., and Cash, K.M., 2008, Survival and migration behavior of juvenile salmonids at McNary Dam, 2006: U. S. Geological Survey, Annual report to the U. S. Army Corps of Engineers, Walla Walla District, Report no. 2006-W68SBV60478899..

Adams, N.S., Rondorf, D.W, Evans, S.D., Kelly, J.E., and Perry, R.W., 1998, Effects of surgically and gastrically implanted radio transmitters on growth and feeding behavior of juvenile Chinook salmon: Transactions of the American Fisheries Society, v. 127, p. 128-136.

Akaike, H., 1973, Information theory and an extension of the maximum likelihood principle:

International Symposium on Information Theory, 2nd, Budapest, 1973 [Proceedings], p. 267-281.

Burnham, K.P., and Anderson, D.R., 1998, Model selection and inference - a practical informationtheoretic approach: New York, Springer-Verlag, 353 p. 
Burnham, K.P., Anderson, D.R., White, G.C., Brownie, C., and Pollock, K.H., 1987, Design and analysis methods for fish survival experiments based on release-recapture: American Fisheries Society Monograph, no. 5, 437 p.

Collett, D., 2003, Modeling Binary Data: Boca Raton, Florida, Chapman \& Hall/CRC, 387 p.

Collis, K., Roby, D.D., Craig, D.P., Ryan, B.A., and Ledgerwood, R.D., 2001, Colonial waterbird predation on juvenile salmonids tagged with passive integrated transponders in the Columbia River estuary - Vulnerability of different salmonid species, stocks, and rearing types: Transactions of the American Fisheries Society, v. 130, p. 385-396.

Cormack, R.M., 1964, Estimates of survival from the sighting of marked animals: Biometrika, v. 51, p. 429-438.

Jolly, G.M., 1965, Explicit estimates from capture-recapture data with both death and immigrationstochastic model: Biometrika, v. 52, p. 225-247.

Laake, J., and Rextad, E., 2008, Appendix C, in Cooch, E. and White, G., Program MARK-A 'gentle' introduction, accessed August 19, 2011 at http://www.phidot.org/software/mark/docs/book/.

Lebreton, J.D., Burnham, K.P., Clobert, J., and Anderson, D.R., 1992, Modeling survival and testing biological hypotheses using marked animals - a unified approach with case studies: Ecological Monographs, v. 62, p. 67-118.

Perry, R.W., Braatz, A.C., Fielding, S.D., Lucchesi, J.N., Plumb, J.M., Adams, N.S., and Rondorf, D.W., 2006, Survival and migration behavior of juvenile salmonids at McNary Dam, 2004: U.S. Geological Survey, Final report to the U. S. Army Corps of Engineers, Walla Walla District, Contract no. W68SBV40271050.

Perry, R.W., Braatz, A.C., Novick, M.S., Lucchesi, J.L., Rutz, G.L., Koch, R.C., Schei, J.S., Adams, N.S., and Rondorf, D.W., 2007, Survival and migration behavior of juvenile salmonids at McNary Dam, 2005: U. S. Geological Survey, Final report to the U. S. Army Corps of Engineers, Walla Walla District, Contract no. W68SBV50407617.

Mesa, M. G., 1994, Effects of multiple acute stressors on the predator avoidance ability and physiology of juvenile Chinook salmon: Transactions of the American Fisheries Society, v. 123, p. 786-793.

Plumb, J. M., Perry, R.W., Adams, N.S., and Rondorf, D.W., 2006, The effects of river impoundment and hatchery rearing on the migration behavior of juvenile steelhead in the Lower Snake River, Washington: North American Journal of Fisheries Management, v. 26, p. 438-452.

R Development Core Team, 2011, R-A language and environment for statistical computing: Vienna, Austria, R Foundation for Statistical Computing, accessed August 19, 2011 at http://www.Rproject.org.

Raymond, H. L., 1968, Migration rates of hatchery Chinook salmon in relation to flows and impoundments in the Columbia and Snake rivers: Transactions of the American Fisheries Society, v. 97, p. 356-359.

Raymond, H. L., 1979, Effects of dams and impoundments on migrations of juvenile Chinook salmon and steelhead from the Snake River, 1966 to 1975: Transactions of the American Fisheries Society, v. 108 , p. 505-529. 
Seber, G.A.F., 1965, A note on the multiple recapture census: Biometrika, v. 52, p. 249-259.

Skalski, J.R., Smith, S.G., Iwamoto, R.N., Williams, J.G., and Hoffmann, A., 1998, Use of passive integrated transponder tags to estimate survival of migrant juvenile salmonids in the Snake and Columbia Rivers: Canadian Journal of Fisheries and Aquatic Sciences, v. 55, p. 1484-1493.

Vigg, S., Poe, T.P., Prendergast, L.A., and Hansel, H.C., 1991, Rates of consumption of juvenile salmonids and alternative prey fish by northern squawfish, walleyes, smallmouth bass, and channel catfish in John Day Reservoir, Columbia River: Transactions of the American Fisheries Society, v. 120, p. 421-438.

Wedemeyer, G.A., Saunders, R.L., and Clarke W.C., 1980, Environmental factors affecting smoltification and early marine survival of anadromous salmonids: U.S. National Marine Fisheries Service Marine Fisheries Review, v. 42, no. 6, p. 1-14.

White, G. C., and Burnham, K.P., 1999, Program MARK: Colorado State University, accessed August 19, 2011 at http://warnercnr.colostate.edu/ gwhite/mark/mark.htm.

Whitney, R. R., Calvin, L.D., Erho, Jr., M.W., and Coutant, C.C., 1997, Downstream passage for salmon at hydroelectric projects in the Columbia River Basin-Development, installation, and evaluation: Northwest Power Planning Council, Report no. 97-15, 109 p. 


\section{Appendix}

\section{Appendix A: Weekly Survival Estimates}

Table A1. Weekly survival estimates during the spring migrational period for yearling Chinook salmon passing through, and released in the tailrace of, McNary Dam, 2004-09.

[Standard error is in parentheses]

\begin{tabular}{|c|c|c|c|c|c|}
\hline & Turbine & Bypass & Spillway & Tailrace/Control & Forebay \\
\hline \multicolumn{6}{|c|}{2004} \\
\hline Apr 15-21 & NA & NA & NA & NA & NA \\
\hline Apr 22-30 & $0.829(0.068)$ & $0.831(0.052)$ & $0.913(0.049)$ & $0.918(0.034)$ & NA \\
\hline May 1-7 & $0.814(0.068)$ & $0.835(0.060)$ & $0.989(0.037)$ & $0.940(0.034)$ & NA \\
\hline May 8-14 & $0.802(0.108)$ & $0.896(0.051)$ & $0.961(0.041)$ & $1.000\left(3.62 \times 10^{-12}\right)$ & NA \\
\hline May 15-21 & $0.829(0.059)$ & $0.924(0.118)$ & $0.962(0.033)$ & $1.000\left(9.01 \times 10^{-8}\right)^{\mathrm{B}}$ & NA \\
\hline May 22-31 & NA & $0.859(0.112)$ & $0.909(0.053)$ & $0.949(0.069)$ & NA \\
\hline Jun 1-7 & NA & NA & NA & NA & NA \\
\hline \multicolumn{6}{|c|}{2005} \\
\hline Apr 15-21 & NA & NA & NA & NA & NA \\
\hline Apr 22-30 & $0.893(0.035)$ & $0.879(0.029)$ & $0.936(0.023)$ & $0.975(0.012)$ & $0.975(0.010)$ \\
\hline May 1-7 & $0.932(0.041)$ & $0.919(0.032)$ & $0.924(0.026)$ & $0.982(0.010)$ & $0.975(0.009)$ \\
\hline May 8-14 & $0.938(0.043)$ & $0.941(0.025)$ & $0.970(0.015)$ & $0.953(0.014)$ & $0.991(0.005)$ \\
\hline May 15-21 & $0.774(0.075)$ & $0.975(0.015)$ & $0.967(0.016)$ & $0.983(0.010)$ & $0.968(0.008)$ \\
\hline May 22-31 & $0.805(0.048)$ & $0.932(0.020)$ & $0.952(0.021)$ & $0.979(0.012)$ & $0.933(0.013)$ \\
\hline Jun 1-7 & $0.714(0.171)$ & $0.901(0.047)$ & $0.946(0.041)$ & $0.983(0.020)$ & $0.882(0.039)$ \\
\hline \multicolumn{6}{|c|}{2006} \\
\hline Apr 15-21 & NA & NA & NA & NA & NA \\
\hline Apr 22-30 & $0.833(0.108)$ & $0.909(0.067)$ & $0.852(0.057)$ & $0.958(0.047)$ & $0.988(0.012)$ \\
\hline May 1-7 & $0.956(0.042)$ & $0.987(0.019)$ & $0.963(0.018)$ & $0.974(0.015)$ & $0.991(0.005)$ \\
\hline May 8-14 & $0.735(0.076)$ & $0.949(0.025)$ & $0.952(0.017)$ & $0.973(0.011)$ & $0.991(0.006)$ \\
\hline May 15-21 & $0.873(0.040)$ & $0.923(0.028)$ & $0.964(0.014)$ & $0.981(0.011)$ & $0.987(0.006)$ \\
\hline May 22-31 & $0.740(0.075)$ & $0.913(0.041)$ & $0.956(0.015)$ & $0.991(0.009)$ & $0.998(0.003)$ \\
\hline Jun 1-7 & $0.817(0.075)$ & $0.911(0.052)$ & $0.957(0.018)$ & $0.997(0.008)$ & $0.985(0.011)$ \\
\hline
\end{tabular}


Table A1 (continued). Weekly survival estimates during the spring migrational period for yearling Chinook salmon passing through, and released in the tailrace of, McNary Dam, 2004-09.

[Standard error is in parentheses]

\begin{tabular}{|c|c|c|c|c|c|}
\hline & Turbine & Bypass & Spillway & Tailrace/Control & Forebay \\
\hline \multicolumn{6}{|c|}{2007} \\
\hline Apr 15-21 & $0.732(0.102)$ & $1.000\left(1.96 \times 10^{-7}\right)^{2}$ & $0.928(0.046)$ & $0.970(0.031)$ & $1.000^{1}$ \\
\hline Apr 22-30 & $0.826(0.062)$ & $0.794(0.049)$ & $0.919(0.033)$ & $1.000\left(7.11 \times 10^{-7}\right)$ & $1.000^{1}$ \\
\hline May 1-7 & $0.939(0.095)$ & $1.000\left(2.74 \times 10^{-6}\right)^{2}$ & $0.878(0.033)$ & $0.949(0.025)$ & $0.992(0.008)$ \\
\hline May 8-14 & $0.647(0.116)$ & $0.972(0.039)$ & $0.938(0.026)$ & $1.000\left(6.56 \times 10^{-10}\right)$ & $1.000^{1}$ \\
\hline May 15-21 & $0.750(0.088)$ & $0.932(0.069)$ & $0.949(0.039)$ & $1.000\left(3.82 \times 10^{-10}\right)$ & $1.000^{1}$ \\
\hline May 22-31 & $0.912(0.068)$ & $1.000\left(1.23 \times 10^{-6}\right)^{2}$ & $0.951(0.039)$ & $1.000\left(1.11 \times 10^{-6}\right)^{2}$ & $1.000^{1}$ \\
\hline Jun 1-7 & $0.622(0.080)$ & $1.000\left(3.52 \times 10^{-7}\right)^{2}$ & $0.899(0.056)$ & $0.933(0.043)$ & $0.945(0.017)$ \\
\hline Jun 8-14 & NA & $1.000\left(6.43 \times 10^{-6}\right)^{2}$ & NA & NA & NA \\
\hline \multicolumn{6}{|c|}{2008} \\
\hline Apr 15-21 & $0.843(0.090)$ & $0.952(0.083)$ & $0.954(0.047)$ & $1.000^{1}$ & $1.000^{1}$ \\
\hline Apr 22-30 & $0.908(0.049)$ & $0.916(0.036)$ & $0.938(0.022)$ & $0.980(0.013)$ & $1.000^{1}$ \\
\hline May 1-7 & $0.963(0.035)$ & $0.981(0.027)$ & $0.906(0.028)$ & $0.989(0.011)$ & $1.000^{1}$ \\
\hline May 8-14 & $1.000^{1}$ & $0.968(0.021)$ & $0.930(0.028)$ & $0.966(0.017)$ & $0.995(0.006)$ \\
\hline May 15-21 & $0.914(0.107)$ & $0.999(0.028)^{2}$ & $0.960(0.018)$ & $0.997(0.012)$ & $1.000^{1}$ \\
\hline May 22-31 & $0.914(0.107)$ & $0.987(0.046)$ & $0.978(0.015)$ & $1.000(0.013)^{2}$ & $0.997(0.004)$ \\
\hline Jun 1-7 & NA & $0.900(0.140)$ & $0.962(0.029)$ & $1.000\left(1.11 \times 10^{-6}\right)^{2}$ & $1.000^{1}$ \\
\hline \multicolumn{6}{|c|}{2009} \\
\hline Apr 15-21 & $1.000^{1}$ & $0.960(0.045)$ & $0.976(0.024)$ & $0.988(0.014)$ & 1.0001 \\
\hline Apr 22-30 & $0.878(0.046)$ & $0.976(0.018)$ & $0.932(0.022)$ & $0.973(0.013)$ & 1.0001 \\
\hline May 1-7 & $0.933(0.046)$ & $0.958(0.022)$ & $0.961(0.022)$ & $0.982(0.011)$ & $0.985(0.011)$ \\
\hline May 8-14 & $0.786(0.078)$ & $0.938(0.025)$ & $0.934(0.025)$ & $0.963(0.016)$ & 1.0001 \\
\hline May 15-21 & $0.838(0.077)$ & $0.940(0.029)$ & $0.975(0.015)$ & $0.964(0.016)$ & $0.985(0.010)$ \\
\hline May 22-31 & $0.808(0.077)$ & $0.957(0.025)$ & $0.963(0.013)$ & $0.929(0.018)$ & $0.996(0.005)$ \\
\hline Jun 1-7 & NA & $0.962(0.054)$ & $1.000^{1}$ & $1.000^{1}$ & 1.0001 \\
\hline
\end{tabular}

${ }^{1}$ Not estimable because survival was 100 percent in the model, therefore, estimate was fixed to 1.00 .

${ }^{2}$ Standard error estimated using sin link because many times it performs better than logit link when estimates are near 1.00 . 
Table A2. Weekly survival estimates during the spring migrational period for juvenile steelhead passing through, and released in the tailrace of, McNary Dam, 2004-09.

[Standard error is in parentheses]

\begin{tabular}{|c|c|c|c|c|c|}
\hline & Turbine & Bypass & Spillway & Tailrace/Control & Forebay \\
\hline \multicolumn{6}{|c|}{2004} \\
\hline Apr 22-30 & $0.778(0.139)$ & $0.906(0.060)$ & $0.972(0.024)$ & $0.902(0.032)$ & NA \\
\hline May 1-7 & $0.714(0.121)$ & $0.919(0.042)$ & $0.929(0.021)$ & $0.925(0.025)$ & NA \\
\hline May 8-14 & $0.889(0.105)$ & $0.905(0.043)$ & $0.953(0.016)$ & $0.971(0.016)$ & NA \\
\hline May 15-21 & $0.900(0.095)$ & $0.916(0.065)$ & $0.934(0.021)$ & $0.925(0.026)$ & NA \\
\hline May 22-31 & $0.600(0.219)$ & $0.997(0.050)$ & $0.885(0.054)$ & $0.922(0.037)$ & NA \\
\hline Jun 1-7 & NA & NA & NA & NA & NA \\
\hline \multicolumn{6}{|c|}{2005} \\
\hline Apr 22-30 & $0.779(0.090)$ & $0.908(0.036)$ & $0.961(0.020)$ & NA & $0.909(0.019)$ \\
\hline May 1-7 & $0.789(0.093)$ & $0.890(0.045)$ & $0.979(0.015)$ & NA & $0.973(0.012)$ \\
\hline May 8-14 & $0.667(0.272)$ & $0.947(0.051)$ & $0.891(0.050)$ & NA & $0.944(0.030)$ \\
\hline May 15-21 & $0.250(0.217)$ & $0.957(0.031)$ & $0.895(0.034)$ & NA & $0.958(0.018)$ \\
\hline May 22-31 & $0.893(0.147)$ & $0.888(0.040)$ & $0.906(0.039)$ & NA & $0.939(0.022)$ \\
\hline Jun 1-7 & $0.500(0.354)$ & NA & $0.909(0.087)$ & NA & $0.750(0.097)$ \\
\hline \multicolumn{6}{|c|}{2006} \\
\hline Apr 22-30 & $0.800(0.178)$ & $1.000\left(3.95 \times 10^{-7}\right)^{2}$ & $1.000\left(2.52 \times 10^{-8}\right)^{2}$ & NA & $0.984(0.018)$ \\
\hline May 1-7 & $0.788(0.114)$ & $1.000\left(5.09 \times 10^{-8}\right)^{2}$ & $1.000(0.018)$ & NA & $0.997(0.005)$ \\
\hline May 8-14 & $1.000^{1}$ & $0.970(0.027)$ & $0.983(0.022)$ & NA & $1.000^{1}$ \\
\hline May 15-21 & $0.905(0.064)$ & $0.939(0.055)$ & $0.961(0.020)$ & NA & $0.994(0.006)$ \\
\hline May 22-31 & $1.000(0.001)$ & $0.980(0.019)$ & $0.990(0.015)$ & NA & $0.994(0.006)$ \\
\hline Jun 1-7 & $0.400(0.219)$ & NA & $1.000^{1}$ & NA & NA \\
\hline \multicolumn{6}{|c|}{2007} \\
\hline Apr 22-30 & $0.500(0.158)$ & $0.900(0.054)$ & $0.947(0.020)$ & NA & $1.000^{1}$ \\
\hline May 1-7 & $0.875(0.116)$ & $0.882(0.055)$ & $0.947(0.024)$ & NA & $0.993(0.008)$ \\
\hline May 8-14 & $1.000^{1}$ & $0.887(0.125)$ & $0.954(0.035)$ & NA & $1.000^{1}$ \\
\hline May 15-21 & $0.500(0.354)$ & $1.000\left(4.43 \times 10^{-6}\right)^{2}$ & $0.923(0.039)$ & NA & $0.987(0.013)$ \\
\hline May 22-31 & $0.500(0.204)$ & $0.885(0.062)$ & $0.823(0.044)$ & NA & $0.978(0.016)$ \\
\hline Jun 1-7 & $\mathrm{N}^{3}$ & $0.965(0.303)$ & $0.873(0.054)$ & NA & $1.000^{1}$ \\
\hline Jun 8-14 & $0.667(0.272)$ & NA & NA & NA & NA \\
\hline
\end{tabular}


Table A2 (continued). Weekly survival estimates during the spring migrational period for juvenile steelhead passing through, and released in the tailrace of, McNary Dam, 2004-09.

[Standard error is in parentheses]

\begin{tabular}{lccccl}
\hline & Turbine & Bypass & Spillway & Tailrace/Control & Forebay \\
\hline Apr 15-21 & & \multicolumn{2}{c}{2008} & & \\
Apr 22-30 & $0.500(0.250)$ & NA & $1.000^{1}$ & $0.973(0.041)$ & $1.000^{1}$ \\
May 1-7 & $0.750(0.191)$ & $1.000(0.025)^{2}$ & $0.988(0.015)$ & $0.937(0.020)$ & $0.987(0.008)$ \\
May 8-14 & $0.778(0.098)$ & $1.000(0.022)^{2}$ & $0.998(0.013)$ & $0.994(0.016)$ & $0.995(0.005)$ \\
May 15-21 & $0.600(0.219)$ & $0.960(0.051)$ & $0.932(0.027)$ & $0.967(0.023)$ & $1.000^{1}$ \\
May 22-31 & $0.750(0.217)$ & $1.000^{\mathrm{A}}$ & $0.939(0.023)$ & $1.000(0.0002)$ & $1.000^{1}$ \\
Jun 1-7 & $0.842(0.084)$ & $1.000\left(5.43 \times 10^{-9}\right)^{2}$ & $1.000(0.050)$ & $0.945(0.026)$ & $1.000^{1}$ \\
\hline & $\mathrm{NA}$ & $\mathrm{NA}$ & $0.956(0.043)$ & $0.967(0.054)$ & $1.000^{1}$ \\
\hline Apr 15-21 & $0.800(0.178)$ & $\mathrm{NA}$ & 2009 & & $1.000^{1}$ \\
Apr 22-30 & $0.889(0.074)$ & $0.985(0.015)$ & $0.979(0.010)$ & $0.954(0.015)$ & $1.000^{1}$ \\
May 1-7 & $0.667(0.192)$ & $0.965(0.026)$ & $0.918(0.025)$ & $0.954(0.019)$ & $1.000^{1}$ \\
May 8-14 & $0.750(0.125)$ & $0.977(0.022)$ & $0.914(0.026)$ & $0.962(0.018)$ & $1.000^{1}$ \\
May 15-21 & $0.600(0.219)$ & $0.946(0.032)$ & $0.949(0.020)$ & $0.918(0.025)$ & $0.964(0.018)$ \\
May 22-31 & $0.778(0.139)$ & $0.927(0.040)$ & $0.964(0.014)$ & $0.951(0.017)$ & $0.994(0.007)$ \\
Jun 1-7 & NA & NA & $0.980(0.023)$ & $1.000^{1}$ & $1.000^{1}$ \\
\hline
\end{tabular}

${ }^{1}$ Not estimable because survival was 100 percent in the model, therefore, estimate was fixed to 1.00.

${ }^{2}$ Standard error estimated using sin link because many times it performs better than logit link when estimates are near 1.00 .

${ }^{3}$ Detection probability was equal to 0.00 , therefore, a survival estimate could not be determined. 
Table A3. Weekly survival estimates during the spring migrational period for subyearling Chinook salmon passing through, and released in the tailrace of, McNary Dam, 2004-09.

[Standard Error in parentheses]

\begin{tabular}{|c|c|c|c|c|c|}
\hline & Turbine & Bypass & Spillway & Tailrace/Control & Forebay \\
\hline \multicolumn{6}{|c|}{2004} \\
\hline Jun $15-21$ & NA & NA & NA & NA & NA \\
\hline Jun $22-30$ & NA & NA & NA & NA & NA \\
\hline Jul 1-7 & $0.731(0.055)$ & $0.773(0.053)$ & NA & $0.898(0.030)$ & NA \\
\hline Jul 8-14 & $0.718(0.051)$ & $0.813(0.041)$ & NA & $0.843(0.028)$ & NA \\
\hline Jul 15-21 & $0.611(0.043)$ & $0.654(0.056)$ & NA & $0.781(0.032)$ & NA \\
\hline Jul 22-31 & $0.697(0.059)$ & $0.650(0.073)$ & NA & $0.873(0.039)$ & NA \\
\hline \multicolumn{6}{|c|}{2005} \\
\hline Jun $15-21$ & NA & NA & NA & NA & \\
\hline Jun 22-30 & $0.772(0.063)$ & $0.807(0.038)$ & $0.883(0.052)$ & $0.865(0.026)$ & $0.967(0.018)$ \\
\hline Jul 1-7 & $0.546(0.150)$ & $1.00(0.0005)$ & $0.886(0.027)$ & $0.921(0.029)$ & $0.948(0.018)$ \\
\hline Jul 8-14 & $0.800(0.207)$ & $0.857(0.138)$ & $0.920(0.023)$ & $0.888(0.027)$ & $0.901(0.023)$ \\
\hline Jul 15-21 & $0.833(0.088)$ & $0.738(0.147)$ & $0.873(0.025)$ & $0.915(0.025)$ & $0.924(0.022)$ \\
\hline Jul 22-31 & $0.667(0.157)$ & NA & $0.952(0.023)$ & $0.896(0.033)$ & $0.947(0.026)$ \\
\hline \multicolumn{6}{|c|}{2006} \\
\hline Jun $15-21$ & NA & NA & $1.000^{1}$ & $0.980(0.019)$ & $1.000^{1}$ \\
\hline Jun $22-30$ & $0.823(0.031)$ & $0.921(0.020)$ & $0.951(0.015)$ & $0.970(0.011)$ & $0.992(0.004)$ \\
\hline Jul 1-7 & $0.723(0.053)$ & $0.871(0.049)$ & $0.919(0.023)$ & $0.934(0.019)$ & $0.992(0.005)$ \\
\hline Jul 8-14 & $0.644(0.057)$ & $0.921(0.059)$ & $0.957(0.020)$ & $0.901(0.023)$ & $0.986(0.008)$ \\
\hline Jul 15-21 & $0.679(0.050)$ & $0.824(0.075)$ & $0.946(0.026)$ & $0.888(0.030)$ & $0.987(0.009)$ \\
\hline Jul 22-31 & NA & NA & NA & NA & $0.900(0.095)$ \\
\hline \multicolumn{6}{|c|}{2007} \\
\hline Jun $15-21$ & $0.714(0.121)$ & NA & $1.000(0.002)$ & $1.000(0.0003)$ & $0.941(0.040)$ \\
\hline Jun $22-30$ & $0.763(0.055)$ & $0.869(0.044)$ & $0.900(0.031)$ & $0.943(0.037)$ & $0.991(0.007)$ \\
\hline Jul 1-7 & $1.000(0.002)$ & $0.942(0.105)$ & $0.893(0.052)$ & $0.912(0.052)$ & $0.980(0.010)$ \\
\hline Jul 8-14 & $0.693(0.072)$ & $0.941(0.072)$ & $0.840(0.036)$ & $0.919(0.030)$ & $0.994(0.010)$ \\
\hline Jul 15-21 & $0.620(0.059)$ & $0.737(0.060)$ & $0.813(0.032)$ & $0.819(0.035)$ & $0.990(0.009)$ \\
\hline Jul 22-31 & $0.617(0.104)$ & $0.978(0.119)$ & $0.802(0.051)$ & $0.776(0.057)$ & $0.997(0.012)$ \\
\hline
\end{tabular}


Table A3 (continued). Weekly survival estimates during the spring migrational period for subyearling Chinook salmon passing through, and released in the tailrace of, McNary Dam, 2004-09.

[Standard Error in parentheses]

\begin{tabular}{lcccll}
\hline & \multicolumn{1}{c}{ Turbine } & Bypass & Spillway & Tailrace/Control & \multicolumn{1}{l}{ Forebay } \\
\hline Jun 15-21 & \multicolumn{1}{l}{ NA } & $0.903(0.113)$ & $0.963(0.043)$ & $0.880(0.074)$ & $1.000^{1}$ \\
Jun 22-30 & $0.993(0.017)$ & $0.885(0.038)$ & $0.968(0.013)$ & $0.971(0.012)$ & $1.000(0.00005)$ \\
Jul 1-7 & $0.790(0.054)$ & $0.933(0.039)$ & $0.949(0.021)$ & $0.965(0.017)$ & $1.000^{1}$ \\
Jul 8-14 & $0.737(0.054)$ & $0.918(0.060)$ & $0.920(0.019)$ & $0.905(0.020)$ & $0.996(0.004)$ \\
Jul 15-21 & $0.673(0.059)$ & $0.838(0.072)$ & $0.923(0.025)$ & $0.885(0.022)$ & $1.000^{1}$ \\
Jul 22-31 & $0.644(0.068)$ & $0.639(0.079)$ & $0.818(0.026)$ & $0.842(0.024)$ & $0.971(0.010)$ \\
\hline & & & 2009 & & \\
\hline Jun 15-21 & NA & NA & $0.952(0.046)$ & $1.000^{1}$ & $1.000^{1}$ \\
Jun 22-30 & $0.893(0.041)$ & $0.962(0.023)$ & $0.957(0.014)$ & $0.980(0.009)$ & $1.000^{1}$ \\
Jul 1-7 & $0.813(0.056)$ & $0.968(0.022)$ & $0.890(0.022)$ & $0.977(0.010)$ & $0.997(0.004)$ \\
Jul 8-14 & $0.824(0.065)$ & $0.880(0.047)$ & $0.907(0.020)$ & $0.921(0.019)$ & $0.992(0.006)$ \\
Jul 15-21 & $0.554(0.066)$ & $0.768(0.068)$ & $0.820(0.032)$ & $0.833(0.028)$ & $0.969(0.012)$ \\
Jul 22-31 & $0.508(0.062)$ & $0.400(0.097)$ & $0.649(0.036)$ & $0.821(0.024)$ & $0.909(0.018)$ \\
\hline
\end{tabular}

${ }^{1}$ Not estimable because survival was 100 percent in the model, therefore, estimate was fixed to 1.00. 
Publishing support provided by the U.S. Geological Survey

Publishing Network, Tacoma Publishing Service Center

For more information concerning the research in this report, contact the Director, Western Fisheries Research Center

U.S. Geological Survey

6505 NE 65th Street

Seattle, Washington 98115

http://wfrc.usgs.gov/ 
